Supporting Information

\title{
Characterization of Tailoring Methyltransferases Involved in K-41A Biosynthesis: Modulating Methylation to Improve K-41A Anti-infective Activity
}

Jiang Chen, ${ }^{\dagger, \star, \S}$ Chun Gui, ${ }^{\dagger, \star, \S}$ Qiuyu Wei, ${ }^{*}$ Jie Liu, ${ }^{\#}$ Li Ye, ${ }^{\#}$ Xinpeng Tian, ${ }^{\dagger}$ Yu-Cheng Gu, ${ }^{\perp}$ Qinglian $\mathrm{Li},{ }^{\dagger}$ Jianhua $\mathrm{Ju}^{*}, \dagger,+$

${ }^{\dagger}$ CAS Key Laboratory of Tropical Marine Bio-resources and Ecology, Guangdong Key Laboratory of Marine Materia Medica, RNAM Center for Marine Microbiology, South China Sea Institute of Oceanology, Chinese Academy of Sciences, 164 West Xingang Road, Guangzhou, 510301, China

${ }^{\ddagger}$ College of Oceanology, University of Chinese Academy of Sciences, 19 Yuquan Road, Beijing, 100049, China

${ }^{\#}$ Guangxi Collaborative Innovation Center for Biomedicine, Life Sciences Institute, Guangxi Medical University, Nanning, 530021, Guangxi, China

${ }^{\perp}$ Syngenta Jealott's Hill International Research Centre, Bracknell, Berkshire RG42 6EY, UK

${ }^{\S}$ These authors contributed equally to this work. 


\begin{tabular}{|c|c|c|}
\hline Item Name & Contents/Description & Page \\
\hline \multirow{8}{*}{$\begin{array}{l}\text { Experimental } \\
\text { Methods }\end{array}$} & General Experimental Procedures & S3 \\
\hline & Genome sequencing assembly and DNA sequence analyses & S3 \\
\hline & Construction of gene mutant strains & S3-S4 \\
\hline & $\begin{array}{l}\text { Small-scale fermentation and analyses of wild-type (WT) and } \\
\text { mutant strains }\end{array}$ & S4 \\
\hline & Production, isolation and structure elucidation of compounds 3-11 & S4-S7 \\
\hline & Methanol immersion tests & S7 \\
\hline & Anti-HIV activity detection of nine derivatives & S7 \\
\hline & Antibacterial activity assessments for each derivative & S7-S8 \\
\hline Table S1 & $\begin{array}{l}\text { Deduced functions of ORFs in the polyether antibiotic K-41A } \\
\text { biosynthetic gene cluster (BGC) }\end{array}$ & S9-S10 \\
\hline Table S2 & $\begin{array}{l}\text { The }{ }^{1} \mathrm{H} \text { and }{ }^{13} \mathrm{C} \text { NMR data for compounds } 3 \text { and } 4 \text { in } \mathrm{CDCl}_{3}(\delta \text { in } \\
\text { ppm, } J \text { in } \mathrm{Hz} \text { ) }\end{array}$ & S11 \\
\hline Table S3 & $\begin{array}{l}\text { The }{ }^{1} \mathrm{H} \text { and }{ }^{13} \mathrm{C} \text { NMR data for compounds } 5 \text { and } \mathbf{6} \text { in } \mathrm{CDCl}_{3}(\delta \text { in } \\
\text { ppm, } J \text { in } \mathrm{Hz})\end{array}$ & $\mathrm{S} 12$ \\
\hline Table S4 & $\begin{array}{l}\text { The }{ }^{1} \mathrm{H} \text { and }{ }^{13} \mathrm{C} \text { NMR data for compounds } 7 \text { and } 8 \text { in } \mathrm{CDCl}_{3}(\delta \text { in } \\
\text { ppm, } J \text { in } \mathrm{Hz})\end{array}$ & $\mathrm{S} 13$ \\
\hline Table S5 & $\begin{array}{l}\text { The }{ }^{1} \mathrm{H} \text { and }{ }^{13} \mathrm{C} \text { NMR data for compounds } 9 \text { and } \mathbf{1 0} \text { in } \mathrm{CDCl}_{3}(\delta \text { in } \\
\text { ppm, } J \text { in } \mathrm{Hz})\end{array}$ & $\mathrm{S} 14$ \\
\hline Table S6 & $\begin{array}{l}\text { The }{ }^{1} \mathrm{H} \text { and }{ }^{13} \mathrm{C} \text { NMR data for compound } 11 \text { in } \mathrm{CDCl}_{3}(\delta \text { in ppm, } J \\
\text { in } \mathrm{Hz} \text { ) }\end{array}$ & S15 \\
\hline Table S7 & Antibacterial activities of compounds 3-11 & S16 \\
\hline Table S8 & Strains or plasmids used and constructed in this study & S17 \\
\hline Table S9 & Primers used in this study & $\mathrm{S} 18$ \\
\hline Figure S1 & $\begin{array}{l}\text { Previously reported polyether compounds as well as the polyether } \\
\text { antibiotics K-41A and K-41Am isolated from Streptomyces sp. } \\
\text { SCSIO } 01680\end{array}$ & S19 \\
\hline Figure S2 & $\begin{array}{l}\text { pak4 gene disruption in Streptomyces sp. SCSIO } 01680 \text { via } \\
\text { PCR-targeting technique }\end{array}$ & S19 \\
\hline Figure S3 & $\begin{array}{l}\text { HR-ESI-LC-MS of fermentation extract from WT Streptomyces } \\
\text { sp. SCSIO } 01680 \text { and ketosynthase gene (pak4) disrupted mutant } \\
\text { strain Streptomyces sp. SCSIO 01680/_pak4 }\end{array}$ & S20 \\
\hline Figure S4 & The biological activity test against Micrococcus luteus & $\mathrm{S} 21$ \\
\hline Figure S5 & $\begin{array}{l}\text { Alignment of sequences of PKS domains from polyether antibiotic } \\
\text { K-41A BGC }\end{array}$ & $\mathrm{S} 22$ \\
\hline Figure S6-S10 & $\begin{array}{l}\text { Disruption of five methyltransferase genes in Streptomyces sp. } \\
\text { SCSIO } 01680 \text { via PCR-targeting technique }\end{array}$ & $\mathrm{S} 23-\mathrm{S} 25$ \\
\hline
\end{tabular}




\begin{tabular}{|c|c|c|}
\hline Figure S11 & $\begin{array}{l}\text { HR-ESI-LC-MS profiles of the fermentation extracts of five } \\
\text { methyltransferase gene mutant strains }\end{array}$ & S26-S27 \\
\hline Figure S12 & $\begin{array}{l}\text { The TLC and HR-ESI-MS analysis results of methanol immersion } \\
\text { tests }\end{array}$ & $\mathrm{S} 28$ \\
\hline Figure S13 & $\begin{array}{l}\text { Plausible interconversion mechanisms (methanolysis and } \\
\text { hydrolysis) of } \mathbf{6 / 7} \text { and } \mathbf{9 / 1 0}\end{array}$ & $\mathrm{S} 28$ \\
\hline Figure S14 & $\begin{array}{l}\text { The TLC detection (iodine staining) of partially purified fractions } \\
\text { and finally purified compounds } \mathbf{3 - 1 1}\end{array}$ & $\mathrm{S} 29$ \\
\hline Figure S15 & HR-ESI-MS spectrum of compound 3 in $\mathrm{MeOH}$ & $\mathrm{S} 30$ \\
\hline Figure S16 & IR spectrum of compound $\mathbf{3}$ & $\mathrm{S} 30$ \\
\hline Figures S17-S23 & $1 \mathrm{D}$ and 2D NMR spectra of compound $\mathbf{3}$ in $\mathrm{CDCl}_{3}$ & S31-S37 \\
\hline Figure S24 & HR-ESI-MS spectrum of compound 4 in $\mathrm{MeOH}$ & S38 \\
\hline Figure S25 & IR spectrum of compound 4 & $\mathrm{~S} 38$ \\
\hline Figures S26-S32 & 1D and 2D NMR spectra of compound 4 in $\mathrm{CDCl}_{3}$ & S39-S45 \\
\hline Figure S33 & HR-ESI-MS spectrum of compound 5 in $\mathrm{MeOH}$ & S46 \\
\hline Figure S34 & IR spectrum of compound $\mathbf{5}$ & S46 \\
\hline Figures S35-S41 & $1 \mathrm{D}$ and 2D NMR spectra of compound 5 in $\mathrm{CDCl}_{3}$ & S47-S53 \\
\hline Figure S42 & HR-ESI-MS spectrum of compound 6 in $\mathrm{MeOH}$ & S54 \\
\hline Figure S43 & IR spectrum of compound $\mathbf{6}$ & S54 \\
\hline Figures S44-S50 & $1 \mathrm{D}$ and 2D NMR spectra of compound 6 in $\mathrm{CDCl}_{3}$ & S55-S61 \\
\hline Figure S51 & HR-ESI-MS spectrum of compound 7 in $\mathrm{MeOH}$ & S62 \\
\hline Figure S52 & IR spectrum of compound 7 & S62 \\
\hline Figures S53-S59 & 1D and 2D NMR spectra of compound 7 in $\mathrm{CDCl}_{3}$ & S63-S69 \\
\hline Figure S60 & HR-ESI-MS spectrum of compound 8 in $\mathrm{MeOH}$ & $\mathrm{S} 70$ \\
\hline Figure S61 & IR spectrum of compound $\mathbf{8}$ & S70 \\
\hline Figures S62-S68 & 1D and 2D NMR spectra of compound 8 in $\mathrm{CDCl}_{3}$ & S71-S77 \\
\hline Figure S69 & HR-ESI-MS spectrum of compound 9 in $\mathrm{MeOH}$ & S78 \\
\hline Figure S70 & IR spectrum of compound 9 & S78 \\
\hline Figures S71-S77 & 1D and 2D NMR spectra of compound 9 in $\mathrm{CDCl}_{3}$ & S79-S85 \\
\hline Figure S78 & HR-ESI-MS spectrum of compound $\mathbf{1 0}$ in $\mathrm{MeOH}$ & S86 \\
\hline Figure S79 & IR spectrum of compound $\mathbf{1 0}$ & S86 \\
\hline Figures S80-S86 & $1 \mathrm{D}$ and 2D NMR spectra of compound 10 in $\mathrm{CDCl}_{3}$ & S87-S93 \\
\hline Figure S87 & HR-ESI-MS spectrum of compound 11 in $\mathrm{MeOH}$ & S94 \\
\hline Figure S88 & IR spectrum of compound $\mathbf{1 1}$ & S94 \\
\hline Figures S89-S95 & $1 \mathrm{D}$ and 2D NMR spectra of compound 11 in $\mathrm{CDCl}_{3}$ & S95-S101 \\
\hline- & Supplemental References & S102 \\
\hline
\end{tabular}




\section{General Experimental Procedures}

The bacteria, plasmids and primers used in this study are summarized in Tables S8 and S9. Streptomyces sp. SCSIO 01680 which was isolated from the sediment of the South China Sea was the producer of polyether antibiotic K-41A in this study. One ketosynthase (KS) gene mutant strain was constructed in this study to identify the biosynthetic gene cluster (BGC) of K-41A. Five methyltransferase gene mutant strains were constructed in this study as the producers of demethylated and/or deglycosylated derivatives. The wild-type (WT) and mutant strains were all cultivated in solid modified MS (Murashige and Skoog) medium ( 2\% soy flour, $2 \%$ mannitol, $0.2 \%$ agar, $\mathrm{pH}$ 7.2-7.4) to grow spores, and the modified RA medium including $1 \%$ glucose, $1 \%$ maltose extract, $0.5 \%$ corn flour, $2 \%$ soluble starch, $1 \%$ maltose, $3 \%$ crude sea salt and $0.2 \% \mathrm{CaCO}_{3}, \mathrm{pH}$ 7.2-7.4 was used for large-scale fermentation, the optimum culture temperature was $28{ }^{\circ} \mathrm{C}$. E. coli and Micrococcus luteus were cultivated on LB (Luria-Bertani) medium (0.5\% yeast extract, $1 \%$ trytone, $1 \% \mathrm{NaCl}, \mathrm{pH} 7.0-7.4)$ at $37{ }^{\circ} \mathrm{C}$. The work concentrations of antibiotics used in this study were: $50 \mu \mathrm{g} / \mathrm{mL}$ apramycin (Apr), $50 \mu \mathrm{g} / \mathrm{mL}$ kanamycin (Kan), $50 \mu \mathrm{g} / \mathrm{mL}$ trimethoprim (TMP) and $25 \mu \mathrm{g} / \mathrm{mL}$ chloroamphenicol (Cml). All primers were synthesized by Sangon Biotech (Shanghai) Co. Ltd.

Column chromatography (CC) was performed using silica gel (100-200 mesh, 200-300 mesh; Jiangpeng Silica gel development Inc., Shandong, China). Thin layer chromatography (TLC) was conducted with precoated glass plates (0.1-0.2 mm; silica gel GF254, 10-40 nm, Jiangpeng, China). Preparative TLC was conducted with precoated glass plates $(0.4-0.5 \mathrm{~mm}$; silica gel HSGF254, $8 \pm$ $2 \mathrm{~mm} \geq 80 \%$, Jiangpeng, China). These chromatography techniques were used for compounds isolation. NMR spectra were recorded on a Bruker Avance 500 or a Bruker Avance 700 spectrometer (Bruker, Billerica, MA, USA). Carbon signals and the residual proton signals of $\mathrm{CDCl}_{3}$ $\left(\delta_{\mathrm{C}} 77.16\right.$ and $\left.\delta_{\mathrm{H}} 7.26\right)$ were used for calibration. Coupling constants $(J)$ are given in Hz. Molecular weights were detected by HR-ESI-MS (Bruker micro TOF-QII mass spectrometer).

All reagents used in this study were purchased from Sigma-Aldrich Company Ltd, Toronto Research Chemicals Inc, Sangon Biotech (Shanghai) Co. Ltd, Amatek Scientific Co. Ltd and Shanghai Macklin Biochemical Co. Ltd.

\section{Genome sequencing assembly and DNA sequence analysis}

The whole genome of Streptomyces sp. SCSIO 01680 was sequenced and assembled by Shanghai Biozeron Technology Co. Ltd using Illumina and PacBio sequencing technology. Analysis and annotations of secondary metabolite gene clusters were accomplished by online antiSMASH software (https://antismash.secondarymetabolites.org). Online bioinformatics software BLAST (https://blast.ncbi.nlm.nih.gov/Blast.cgi) was used for sequence alignment and function prediction of genes in the cluster. The PKS/NRPS Analysis Web-site (http://nrps.igs.umaryland.edu) was used to identify domains contained in each gene, then multiple gene sequences of domains were submitted to the Clustalx 1.83 software for complete alignment and the results were refined by online ESPript 3.0 program (http://espript.ibcp.fr/ESPript/cgi-bin/ESPript.cgi). The conserved sequences and key amino acid residues were marked manually.

\section{Construction of gene mutant strains}

One KS gene (pak4) and five methyltransferase gene (pak13, pak15, pak20, pak31 and pak38) contained in pak cluster were inactivated by $\lambda$-RED recombination technology according to the REDIRECT protocol [1]. The cosmid 20-5G containing pak4 gene, cosmid 17-4E and 16-11G 
covering the five methyltransferase gene were selected for gene disruption. The apramycin resistance gene oriT/aac(3)IV cassettes obtained by PCR (primers designed for gene inactivation were listed in Table S9) were used to partially replace the target genes [2]. The constructed mutant cosmids were transferred into nonmethylating E. coli ET12567/pUZ8002, then with the help of non-transmissible plasmid pUZ8002 the mutant cosmids were introduced into Streptomyces sp. SCSIO 01680 during conjugation process [3,4]. The mixture of E. coli and streptomycete spores was spreaded on the solid modified MS medium containing $20 \mathrm{mM} \mathrm{Mg}{ }^{2+}$, after $16-20 \mathrm{~h}$ incubation, the

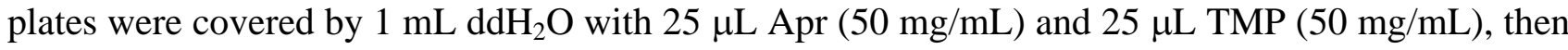
continuous incubation to generate exconjugants. Two kinds of MS solid medium, one contained apramycin and trimethoprim, another contained additional kanamycin, were used for selecting correct conjugants which were apramycin-resistant but kanamycin-sensitive. For further confirmation, PCR was performed and the double exchange mutant strains were identified and preserved.

\section{Small-scale fermentation and analyses of WT and mutant strains}

The WT strain Streptomyces sp. SCSIO 01680 and mutant strains Streptomyces sp. SCSIO 01680/Apak4, $\Delta p a k 13, \Delta p a k 15, \Delta p a k 20, \Delta p a k 31$ and $\Delta p a k 38$ were incubated on solid modified MS medium for sporulation. Then a portion of mycelium and spores was inoculated into $50 \mathrm{~mL}$ modified RA medium in $250 \mathrm{~mL}$ flask and cultivated in $200 \mathrm{rpm}$ shaker at $28^{\circ} \mathrm{C}$ for $7 \mathrm{~d}$. Each culture was extracted with equal volume of butanone, organic phase was evaporated to dryness then redissolved in $500 \mu \mathrm{L} \mathrm{MeOH}$. Because polyether antibiotic K-41A and its congeners have no UV absorption and cannot be detected by HPLC, the extracts were analyzed by biological activity tests using the disc diffusion assay (Figure S4). $5 \mu \mathrm{L}$ extract was added to each disc with Micrococcus luteus as the indicator. HR-ESI-LC-MS analysis was carried out using Bruker micro TOF-QII mass spectrometer to extract ion flows. The mobile phase of LC-MS comprises solvents $\mathrm{A}$ and $\mathrm{B}$, solvent $\mathrm{A}$ is $\mathrm{ddH}_{2} \mathrm{O}$ supplemented with $0.1 \%$ TFA and solvent $\mathrm{B}$ is $\mathrm{CH}_{3} \mathrm{CN}$ supplemented with $0.1 \%$ TFA. Samples were eluted with a linear gradient from $20 \%$ to $80 \%$ solvent B in 20 min, followed by $80 \%$ to $100 \%$ solvent B for $1 \mathrm{~min}$, then eluted with $100 \%$ solvent B for $4 \mathrm{~min}$, all at a flow rate of $1 \mathrm{~mL} / \mathrm{min}$.

\section{Production, isolation and structure elucidation of compounds 3-11.}

In order to characterize the structures of K-41A's demethylated and/or deglycosylated derivatives

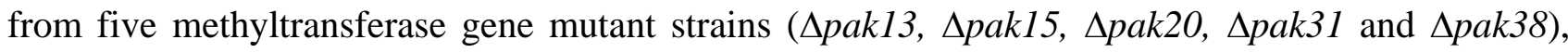
large-scale fermentation of each mutant on a $16 \mathrm{~L}$ scale was carried out. A portion of mycelium and spores was seeded into $50 \mathrm{~mL}$ modified RA medium in a $250 \mathrm{~mL}$ flask. After incubation in a shaker at $28^{\circ} \mathrm{C}, 200 \mathrm{rpm}$ for $2 \mathrm{~d}, 25 \mathrm{~mL}$ of each culture was transferred into a $1 \mathrm{~L}$ flask containing $200 \mathrm{~mL}$ modified RA medium and then cultured at $28^{\circ} \mathrm{C}$ and $200 \mathrm{rpm}$ for $7 \mathrm{~d}$. The growth culture from each mutant was centrifuged at $3900 \mathrm{~g}$ for $25 \mathrm{~min}$ to yield supernatant and mycelium cake. The supernatant and mycelium were all extracted by equal volume of ethyl acetate for three times. The organic extracts were combined and concentrated to dryness, yielding a syrupy residue.

The residue from the $\Delta p a k 13$ mutant strain was subjected to a normal phase silica gel (100-200 mesh) column eluted with petroleum ether-ethyl acetate (100:0, 80:20, 60:40, 40:60, 20:80, 0:100, $\mathrm{v} / \mathrm{v}, 500 \mathrm{~mL}$ volume for each fraction) and ethyl acetate-MeOH (98:2, 96:4, 94:6, 92:8, 90:10, 80:20, $50: 50, \mathrm{v} / \mathrm{v}, 500 \mathrm{~mL}$ volume for each fraction) to give 13 fractions (AFr.1-AFr.13). The antibacterial activity was detected by disc diffusion assay using Micrococcus luteus as indicator; $5 \mu \mathrm{L}$ of each fraction was added to a filter paper sheet, and the inhibition zone was observed after $12 \mathrm{~h}$ cultivation 
at $28{ }^{\circ} \mathrm{C}$. The active fractions AFr.3-AFr.5 were then combined and subjected to medium pressure liquid chromatography (MPLC, CHEETAHTM MP 200) with an ODS column eluted in a linear gradient of $0 \%$ to $100 \%$ B phase (A phase: $\mathrm{ddH}_{2} \mathrm{O}$, B phase: $\mathrm{CH}_{3} \mathrm{CN}$ ) over 120 min, at a flow rate of $10 \mathrm{~mL} / \mathrm{min}$, to give 12 fractions (BFr.1-BFr.12). With the guidance of antibacterial activity test and TLC analysis, the active ingredient BFr.7 was subjected to a normal phase silica gel (200-300 mesh) column eluted with $\mathrm{CHCl}_{3}-\mathrm{MeOH}$ (100:0, 99:1, 98:2, 96:4, 94:6, 92:8, 9:1, 8:2, 5:5, 0:100, v/v, 300 $\mathrm{mL}$ volume for each fraction) to give 10 fractions (CFr.1-CFr.10). The active fraction CFr.3 was separated by preparative TLC using a chromatographic condition of $\mathrm{CHCl}_{3}-\mathrm{MeOH}(92 / 8)$ to afford compounds $3(10.0 \mathrm{mg}, \mathrm{Rf}=0.58)$ and $4(65.0 \mathrm{mg}, \mathrm{Rf}=0.82)$ (Figure S14A).

The residue from the $\Delta$ pak15 mutant was subjected to a normal phase silica gel (100-200 mesh) column eluted with petroleum ether-ethyl acetate (100:0, 80:20, 60:40, 40:60, 20:80, 0:100, v/v, 500 $\mathrm{mL}$ volume for each fraction) and ethyl acetate-MeOH (98:2, 96:4, 94:6, 92:8, 90:10, 80:20, 50:50, $\mathrm{v} / \mathrm{v}, 500 \mathrm{~mL}$ volume for each fraction) to give 13 fractions (AFr.1-AFr.13). Based on the results of bioactivity test against Micrococcus luteus and TLC analysis, fraction AFr.6 was then subjected to an ODS column on MPLC and eluted in a linear gradient of $0 \%$ to $100 \%$ B phase (A phase: $\mathrm{ddH}_{2} \mathrm{O}$, B phase: $\mathrm{CH}_{3} \mathrm{CN}$ ), at a flow rate of $10 \mathrm{~mL} / \mathrm{min}$, over $120 \mathrm{~min}$ to give 12 fractions (BFr.1-BFr.12). Fractions BFr.4 and BFr.5 with potent antibacterial activity were purified by preparative TLC using petroleum ether-ethyl acetate (2.5/7.5) as developing solvent, obtaining compound $\mathbf{5}(30.3 \mathrm{mg}, \mathrm{Rf}=$ 0.66) (Figure S14B).

The residue from the $\Delta p a k 20$ mutant strain was subjected to a normal phase silica gel (100-200 mesh) column, and the elution system was the same as that employed for spak15 mutant. With the guidance of the antibacterial activity test against Micrococcus luteus, AFr.4-AFr.7 were combined and further fractionated by MPLC using the same elution condition for $\Delta p a k 15$. Final isolation of BFr.9-BFr.11 was achieved by preparative TLC (petroleum ether/ethyl acetate: 1/9) to afford compounds $6(13.7 \mathrm{mg}, \mathrm{Rf}=0.64)$ and $7(48.1 \mathrm{mg}, \mathrm{Rf}=0.59)($ Figure S14C).

The purification of fermentation extract for the $\Delta p a k 31$ mutant strain was carried out following the same separation steps applied to $\Delta p a k 15$ and $\Delta p a k 20$ mutants. The residue was firstly subjected to a normal phase silica gel (100-200 mesh) column and then an ODS column. The active fractions BFr.7, BFr.9 and BFr.10 were found to contain target compounds. Fraction BFr.7 was separated by preparative TLC (petroleum ether/ethyl acetate: 4/8), yielding compounds 9 (76.5 mg, $\mathrm{Rf}=0.75)$ and $10(49.2 \mathrm{mg}, \mathrm{Rf}=0.61)$. Fractions BFr.9 and BFr.10 were also subjected to preparative TLC (petroleum ether/ethyl acetate: 4/6) to yield compound 8 (54.7 $\mathrm{mg}, \mathrm{Rf}=0.51)$ (Figure S14D).

The same separation process was implemented on $\Delta p a k 38$ as that of $\Delta p a k 15$. The active fraction BFr.10 was purified by preparative TLC under chromatographic condition of petroleum ether-ethyl acetate (5/5), and compound 11 (36.9 $\mathrm{mg}, \mathrm{Rf}=0.71)$ was afforded (Figure S14E).

K-41C (3): white solid; $[\alpha]^{25}(\mathrm{MeOH}):+3.333^{\circ}$; IR (ATR) $v_{\max } 3383,2932,2916,1614,1456$, 1375, 1360, 1020, $943 \mathrm{~cm}^{-1} ;{ }^{1} \mathrm{H}$ and ${ }^{13} \mathrm{C}$ NMR spectroscopic data are listed in Table S2; HRMS (ESI) m/z: [M-H] $]^{-}$Calcd for $\mathrm{C}_{40} \mathrm{H}_{67} \mathrm{O}_{16}$ 803.4435; Found 803.4438. Comparisons of ${ }^{1} \mathrm{H}$ and ${ }^{13} \mathrm{C}$ NMR data (Table S2, Figures S17-S19) with previously reported K-41A datasets revealed that they shared the same structure except for the absence of one methoxyl group at C-5 $\left(\delta_{\mathrm{C}} 61.0, \delta_{\mathrm{H}} 3.56, \mathrm{~s}\right)$ and seven carbons of the 4-methoxy-rhodinose moiety at $\delta_{\mathrm{C}} 102.7$ (C-1'), 30.5 (C-2'), 27.3 (C-3'), 80.4 (C-4'), 56.8 (C-4'-OMe), 74.5 (C-5') and $18.3\left(\mathrm{C}^{\prime} 5^{\prime}-\mathrm{Me}\right)$ in 3. These differences were consistent with MS difference seen between $\mathbf{1}$ and $\mathbf{3}$ (Figure S15). Additionally, 2D NMR data (Figures S20-S23) further confirmed its structure. 3 was a C5-demethylated, C27-deglycosylated derivative of K-41A. 
K-41D (4): white solid; $[\alpha]^{25}(\mathrm{MeOH}):+5.091^{\circ}$; IR (ATR) $v_{\max } 3352,2934,1616,1456,1375$, 1362, 1163, 1065, 1022, 986, $943 \mathrm{~cm}^{-1} ;{ }^{1} \mathrm{H}$ and ${ }^{13} \mathrm{C}$ NMR spectroscopic data are listed in Table S2; HRMS (ESI) $m / z$ : [M-H] $]^{-}$Calcd for $\mathrm{C}_{47} \mathrm{H}_{79} \mathrm{O}_{18}$ 931.5272; Found 931.5279. Compound 4 shared the same structure with K-41A when comparing their NMR data (Figures S26-S32), but 4 lost the C-5 methoxyl moiety $\left(\delta_{\mathrm{C}} 61.0, \delta_{\mathrm{H}} 3.56, \mathrm{~s}\right)$. MS data (Figure S24) was also consistent with this difference. Thus, 4 was determined as a C5-demethylated derivative of K-41A.

K-41E (5): white solid; $[\alpha]^{25}(\mathrm{MeOH}):+3.814^{\circ}$; IR (ATR) $v_{\max } 3337,2938,1614,1454,1377$, 1360, 1163, 1024, 986, $943 \mathrm{~cm}^{-1} ;{ }^{1} \mathrm{H}$ and ${ }^{13} \mathrm{C}$ NMR spectroscopic data are listed in Table S3; HRMS (ESI) $m / z:[\mathrm{M}-\mathrm{H}]^{-}$Calcd for $\mathrm{C}_{47} \mathrm{H}_{79} \mathrm{O}_{18}$ 931.5272; Found 931.5276. MS data of compound 5 revealed a mass deficit of 14 Da relative to K-41A (Figure S33). Comparisons of 1D NMR spectra (Figures $\mathrm{S} 35-\mathrm{S} 37)$ revealed that 5 lacked a ${ }^{13} \mathrm{C}$ chemical shift at $\delta_{\mathrm{C}} 50.8(\mathrm{C}-6-\mathrm{OMe})$. Meanwhile, 2D NMR data (Figures S38-S41) further supported the absence of one OMe group at C-6 $\left(\delta_{\mathrm{C}} 50.8, \delta_{\mathrm{H}} 3.37, \mathrm{~s}\right)$. Consequently, the structure of $\mathbf{5}$ was established as C6-demethylated K-41A.

K-41B (6): white solid; $[\alpha]^{25}(\mathrm{MeOH}):+4.071^{\circ}$; IR (ATR) $v_{\max } 3385,2938,1614,1454,1360$, 1165, 1065, 1030, 988, $947 \mathrm{~cm}^{-1} ;{ }^{1} \mathrm{H}$ and ${ }^{13} \mathrm{C}$ NMR spectroscopic data are listed in Table S3; HRMS (ESI) $m / z:[\mathrm{M}-\mathrm{H}]^{-}$Calcd for $\mathrm{C}_{54} \mathrm{H}_{91} \mathrm{O}_{20}$ 1059.6109; Found 1059.6106. Comparisons of MS and NMR datasets with previously reported data (Table S3, Figures S42, S44-S50) revealed that 6 was the known polyether K-41B.[5]

K-41Bm (7): white solid; $[\alpha]^{25}(\mathrm{MeOH}):+2.109^{\circ}$; IR (ATR) $v_{\max } 3433,2938,1614,1454$, 1371, 1165, 1061, 1034, 988, $949 \mathrm{~cm}^{-1} ;{ }^{1} \mathrm{H}$ and ${ }^{13} \mathrm{C}$ NMR spectroscopic data are listed in Table S4; HRMS (ESI) $m / z$ : [M-H] ${ }^{-}$Calcd for $\mathrm{C}_{55} \mathrm{H}_{93} \mathrm{O}_{20}$ 1073.6266; Found 1073.6264. The NMR data of 7 were almost identical to those of 6 (Table S4, Figures S53-S59) except for additional signals at $\delta_{\mathrm{C}}$ 48.6 and $\delta_{\mathrm{H}}$ 3.10. MS data also revealed 7 to be $14 \mathrm{Da}$ heavier than 6 (Figure S51) and DEPT spectral analysis of both species revealed 7 to possess one Me group more than 6 . The HMBC correlation from the putatively additional $\mathrm{H}$ belonging to a methoxy moeity at $\delta_{\mathrm{H}} 3.10(\mathrm{~s}, \mathrm{H}-\mathrm{OMe})$ to C-29 $\left(\delta_{\mathrm{C}} 100.9\right)$ affirmed a direct connection with C-29 of the PKS skeleton. Thus, 7 was assigned as a C29-methyl ether derivative of K-41B.

K-41F (8): white solid; $[\alpha]^{25}(\mathrm{MeOH}):-2.146^{\circ}$; IR (ATR) $v_{\max } 3433,2940,1728,1614,1456$, 1375, 1360, 1067, 1024, $943 \mathrm{~cm}^{-1} ;{ }^{1} \mathrm{H}$ and ${ }^{13} \mathrm{C}$ NMR spectroscopic data are listed in Table S4; HRMS (ESI) $m / z$ : [M-H] $]^{-}$Calcd for $\mathrm{C}_{47} \mathrm{H}_{77} \mathrm{O}_{18}$ 929.5115; Found 929.5125. Compound 8 was found to possess an extra degree of unsaturation relative to K-41A. The chemical shift for C-4' changed from $\delta_{\mathrm{C}} 80.4$ to $\delta_{\mathrm{C}} 208.7$ (Table S4, Figure S63) and the IR characteristic absorption at $1728 \mathrm{~cm}^{-1}$ (Figure S61) both indicated the presence of a carbonyl carbon. Meanwhile, the carbon signal at $\delta_{\mathrm{C}}$ 56.8 previously diagnostic for the 4'-OMe was absent for $\mathbf{8}$. These data, combined with MS data (Figure S60), made clear that the 4-methoxy-rhodinose of K-41A was replaced in 8 with 2,3,6-trideoxy-4-hexulose. Thus, 8 was identified as a C27-cinerulose substituted derivative of K-41A.

K-41G (9): white solid; $[\alpha]^{25}(\mathrm{MeOH}):+5.680^{\circ}$; IR (ATR) $v_{\max } 3360,2940,1614,1456,1375$, 1360, 1099, 1086, 1022, $945 \mathrm{~cm}^{-1} ;{ }^{1} \mathrm{H}$ and ${ }^{13} \mathrm{C}$ NMR spectroscopic data are listed in Table S5; HRMS (ESI) $m / z$ : [M-H] $]^{-}$Calcd for $\mathrm{C}_{41} \mathrm{H}_{69} \mathrm{O}_{16}$ 817.4591; Found 817.4589. The ${ }^{13} \mathrm{C}$ NMR of 9 lost seven carbon signals, revealing the lack of glycosylation at C-27 (Table S5, Figure S72); MS data (Figure S69) also supported this notion. Ultimately, compound 9 was assigned as a C27-deglycosylated derivative of K-41A.

K-41Gm (10): white solid; $[\alpha]^{25}{ }_{\mathrm{D}}(\mathrm{MeOH}):+3.574^{\circ}$; IR (ATR) $v_{\max } 3428,2940,1614,1460$, 1375, 1184, 1101, 1063, 993, $949 \mathrm{~cm}^{-1} ;{ }^{1} \mathrm{H}$ and ${ }^{13} \mathrm{C}$ NMR spectroscopic data are listed in Table S5; 
HRMS (ESI) m/z: [M-H] Calcd for $\mathrm{C}_{42} \mathrm{H}_{71} \mathrm{O}_{16}$ 831.4748; Found 831.4738. Compound 10 had similar NMR spectra (Table S5, Figures S80-S86) with 9, although 10 possessed one additional carbon signal at $\delta_{\mathrm{C}} 48.6$ consistent with an OMe at C-29 as had been noted for compound 7. Hence, 10 was elucidated as a C27-deglycosylated and C29-methyl ether derivative of K-41A.

K-41H (11): white solid; $[\alpha]^{25}$ D (MeOH): + 3.738 ${ }^{\circ}$; IR (ATR) $v_{\max } 3374,2936,1614,1454,1377$, 1362, 1163, 1026, 986, $943 \mathrm{~cm}^{-1} ;{ }^{1} \mathrm{H}$ and ${ }^{13} \mathrm{C}$ NMR spectroscopic data are listed in Table S6; HRMS (ESI) $\mathrm{m} / z$ : $[\mathrm{M}-\mathrm{H}]^{-}$Calcd for $\mathrm{C}_{47} \mathrm{H}_{79} \mathrm{O}_{18}$ 931.5272; Found 931.5298. Comparative analysis of 1D NMR data (Table S6, Figures S89-S91) for 11, revealing a missing carbon signal at $\delta_{\mathrm{C}} 59.3$ which previously correlated to the 11-OMe moiety of K-41A. 2D NMR data (Figures S92-S95) also strongly suggested that $\mathbf{1 1}$ differed from K-41A by only one methyl group; this was akin to the situation noted earlier in compounds 4 and 5. On the basis of these data, compound 11 was elucidated and assigned as a C11-demethylated derivative of K-41A.

\section{Methanol immersion tests}

Alcoholysis and hydrolysis reactions were performed by incubating compounds 6, 7, 9 and 10 individually in methanol solutions containing 5\% formic acid for $12-24 \mathrm{~h}$ at room temperature. Compounds were also immersed in acid-free $\mathrm{MeOH}$ to serve as control reactions. Reaction mixtures were detected using TLC and HR-ESI-MS (NEG).

\section{Anti-HIV activity detection of nine derivatives}

Anti-HIV assays and cell viability assays were carried out by two virus-cell systems: HIV-1 $1_{\text {IIIB }} /$ TZM-bl and HIV-1 $1_{\text {IIIB }} /$ MT-2. All assays were repeated for three times, the IC $_{50}$ (half inhibitory concentration), $\mathrm{CC}_{50}$ (half cytotoxic concentration) and SI (selectivity index) values were caculated for each testing compound.

For HIV-1 ${ }_{\text {IIIB }} /$ TZM-bl system, TZM-bl cells were firstly plated in 96-well plates at a density of $1 \times 10^{4}$ cells per well and cultured for $24 \mathrm{~h}$. The compound which was serially diluted to eight concentrations along with 1 MOI (multiplicity of infection) HIV-1 $1_{\text {IIIB }}$ virus were added to wells. After incubation for $48 \mathrm{~h}$, the levels of HIV-1 in TZM-bl cells were measured by the Promega Bright-Glo Luciferase Assay.

For HIV-1 $1_{\text {IIIB }} /$ MT- 2 system, firstly, MT- 2 cells with a density of $3.2 \times 10^{4}$ cells/well were plated in 96-well plates, then the serially diluted compounds of different concentrations and $1 \mathrm{MOI}$ HIV-1 $1_{\text {IIIB }}$ virus were added together to each well. After $72 \mathrm{~h}$ incubation, $50 \mu \mathrm{L}$ supernatant from each well was transferred to TZM-bl cell culture in 96-well plates, respectively. After $24 \mathrm{~h}$, the levels of HIV-1 in TZM-bl cells were measured by the Promega Bright-Glo Luciferase Assay.

Cell viability was determined by the CellTiter-Glo ${ }^{\circledR}$ Luminescent Cell Viability Assay (Promega, USA). Cells along with each serially diluted compound were plated in 96-well plates, which was parallel to the anti-HIV assay. After incubation, CellTiter-Glo ${ }^{\circledR}$ Reagent was added to each well, and the luminescent signal was measured using a microplate reader (BioTek, Synergy H1, UK).

\section{Antibacterial activity assessments for each derivative}

Compounds 3-11 were evaluated for their antibacterial activities against Micrococcus luteus, Staphylococcus simulans AKA1, Staphylococcus aureus 16162, Staphylococcus aureus 16339, Staphylococcus aureus ATCC 29213, Staphylococcus aureus (cfr) GDQ6P012P, Bacillus subtilis, and methicillin-resistant Staphylococcus aureus (MRSA) using the two-fold dilution method, referring to previously reported standard methods provided by Clinical and Laboratory Standards 
Institute (CLSI). The lowest concentrations that completely inhibited the growth of bacteria in microdilution wells were detected by unaided eyes for each tested compound, the values were recorded in Table S7. All assays were repeated for three times. 
Table S1. Deduced functions of ORFs in the polyether antibiotic K-41A biosynthetic gene cluster.

\begin{tabular}{|c|c|c|}
\hline ORF & Size $^{a}$ & Proposed function \\
\hline pakl & 262 & Transcriptional regulator \\
\hline pak2 & 255 & Type II thioesterase \\
\hline pak3 & 76 & Hypothetical protein \\
\hline pak4 & 2856 & $\begin{array}{l}\text { PKS I (loading module: KS, AT, ACP; } \\
\text { module 1: KS, AT, DH, KR) }\end{array}$ \\
\hline pak5 & 2242 & $\begin{array}{c}\text { PKS I (module 2: KS, AT, DH, ER, KR } \\
\text { ACP) }\end{array}$ \\
\hline pak6 & 3999 & $\begin{array}{l}\text { PKS I (module } 3: \mathrm{KS}, \mathrm{AT}, \mathrm{DH}, \mathrm{KR}, \mathrm{AC} \\
\text { module 4: KS, AT, DH, ER, KR, ACP) }\end{array}$ \\
\hline pak7 & 660 & PKS I (module 5: KS) \\
\hline pak8 & 3334 & $\begin{array}{l}\text { PKS I (module 5: AT, DH, KR, ACP; } \\
\text { module 6: KS, AT, DH, ER, KR, ACP) }\end{array}$ \\
\hline pak9 & 4110 & $\begin{array}{l}\text { PKS I (module 7: KS, AT, DH, KR, AC) } \\
\text { module 8: KS, AT, DH, ER, KR, ACP) }\end{array}$ \\
\hline paklo & 1669 & PKS I (module 9: KS, AT, KR, ACP) \\
\hline pak11 & 176 & $\begin{array}{l}\text { LLM class flavin-dependent } \\
\text { oxidoreductase }\end{array}$ \\
\hline pak12 & 315 & Thioesterase \\
\hline pak13 & 316 & Methyltransferase \\
\hline pak14 & 417 & Cytochrome P450 \\
\hline pak15 & 280 & Methyltransferase \\
\hline pak16 & 436 & Glycosyltransferase \\
\hline pak17 & 229 & Hypothetical protein \\
\hline pak18 & 404 & Cytochrome P450 \\
\hline
\end{tabular}

Phosphopantetheine-binding

pak19 111 domain-containing protein; Polyketide synthase (ACP)

pak20 $282 \quad$ C11-O-methyltransferase

pak21 $420 \quad$ Cytochrome P450

pak22 $841 \quad$ Beta-ketoacyl synthase (KS)

Closest homolog, origin (protein ID); ID/SI (\%) Homolog

SARP family, Streptomyces nanchangensis NanR1/R

(AAP42853.1 / APP42854.1); 50\%/93\% // 52\%/92\% 2

Type II thioesterase, Actinomadura sp. (QFU19822.1); Lsd4

$$
67.33 \% / 97 \%
$$

MonAX

Hypothetical protein, Streptomyces

hyqroscopicus(WP_060947731.1); 57\%/100\%

Beta-ketoacyl synthase, Streptomyces violaceusniqer Tu 4113(AEM83616.1); 64\%/78\%

NigAI

Beta-ketoacyl synthase, Streptomyces violaceusniqer Tu 4113(AEM83617.1); 62\%/100\%

NigAII

Beta-ketoacyl synthase, Streptomyces violaceusniqer

Tu 4113(AEM83618.1); 53\%/99\%

NigAIII

Laidlomycin polyketide synthase(module 9),

Streptomyces sp. CS684(AFL48520.1); 68\%/96\%

NigAIII;

NanA6

Beta-ketoacyl synthase, Streptomyces sp. 11-1-2(ASQ92848.1); 57\%/99\%

NigAIV

Beta-ketoacyl synthase, Streptomyces sp. 11-1-2(ASQ92847.1); 63\%/99\%

NigAV

Beta-ketoacyl synthase, Streptomyces sp. 11-1-2(ASQ92846.1); 64\%/99\%

NanA6

Flavin reductase, Salinispora

arenicola(WP_018799687.1); 64\%/88\%

Alpha/beta hydrolase, Streptomyces sp.

11-1-2(ASQ92845.1); 49\%/92\%

NanE

SAM-dependent methyltransferase, Streptomyces $\mathrm{sp}$.

CMB-StM0423(WP_101426745.1); 42\%/92\%

Cytochrome P450, Streptomyces sp. NBS 14/10(WP_089509381.1); 59\%/97\%

MfnN

C5-O-methyltransferase, Streptomyces rapamycinicus NRRL 5491(AGP52766.1); 57\%/100\%

NigE

Activator-dependentfamily glycosyltransferase,

Streptomyces puniciscabiei(WP_069778185.1); $72 \% / 98 \%$

Hypothetical protein M271 05575, Streptomyces rapamycinicus NRRL 5491(AGP52741.1); 42\%/70\%

Cytochrome P450, Microbispora rosea(WP_076434135.1); 55\%/95\%

NanG5

NlmOI

$\mathrm{MfnN}$

Phosphopantetheine-binding protein, Streptomyces antioxidans(WP_046084712.1); 52\%/96\%

NigAXI

Methyltransferase domain-containing protein,

Streptomyces malaysiensis(WP_099015918.1); NigE $62 \% / 99 \%$

Cytochrome P450, Nocardiopsis qilva(WP 017617651.1); 52\%/96\%

$\mathrm{MfnN}$

Type I polyketide synthase, Streptomyces sp. NBRC 110028(WP_063787290.1); 56\%/99\% 


\begin{tabular}{|c|c|c|c|c|}
\hline pak23 & 462 & Epoxidase & $\begin{array}{l}\text { FAD-dependent oxidoreductase, Streptomyces } \\
\text { hygroscopicus(WP_079106135.1); 70\%/99\% }\end{array}$ & $\begin{array}{l}\text { LasC; } \\
\text { Lsd18 }\end{array}$ \\
\hline pak24 & 156 & Epoxide hydrolase/Cyclase & $\begin{array}{l}\text { Epoxidase, Streptomyces sp. CS684(AFL48530.1); } \\
54 \% / 85 \%\end{array}$ & Las19 \\
\hline pak25 & 145 & Epoxide hydrolase/Cyclase & $\begin{array}{l}\text { Epoxidase, Streptomyces sp. CS684(AFL48531.1); } \\
49 \% / 96 \%\end{array}$ & $\begin{array}{l}\text { NigBI; } \\
\text { NanI }\end{array}$ \\
\hline pak26 & 365 & $\begin{array}{l}\text { LLM class flavin-dependent } \\
\text { oxidoreductase }\end{array}$ & $\begin{array}{l}\text { LLM class flavin-dependent oxidoreductase, Nocardia } \\
\text { altamirensis(WP 084654485.1);58\%/92\% }\end{array}$ & - \\
\hline pak27 & 498 & Epoxidase & $\begin{array}{c}\text { FAD-dependent oxidoreductase, Streptomyces } \\
\text { hygroscopicus(WP_078638923.1); 65\%/93\% }\end{array}$ & Lsd18 \\
\hline pak28 & 1502 & PKS I (module 14: KS, AT, DH, ACP) & $\begin{array}{c}\text { Beta-ketoacyl synthase, Streptomyces } \\
\text { iranensis(CDR03485.1); 59\%/96\% }\end{array}$ & NigAIV \\
\hline pak29 & 2085 & $\begin{array}{c}\text { PKS I (module 13: KS, AT, DH, unknown } \\
\text { domain, KR, ACP) }\end{array}$ & $\begin{array}{c}\text { Beta-ketoacyl synthase, Streptomyces mobaraensis } \\
\text { NBRC } 13819=\text { DSM 40847(EME96798.1); 57\%/97\% }\end{array}$ & NigAVIII \\
\hline pak30 & 4816 & $\begin{array}{l}\text { PKS I (module 10: KS, AT, KR, ACP; } \\
\text { module 11: KS, AT, KR, ACP; module } \\
\text { 12: KS, AT, KR, ACP) }\end{array}$ & $\begin{array}{l}\text { Beta-ketoacyl synthase, Streptomyces violaceusniger } \\
\text { Tu 4113(AEM84951.1);51\%/99\% }\end{array}$ & NigAVII \\
\hline pak31 & 329 & Putative sugar O-methyltransferase & $\begin{array}{c}\text { Putative sugar O-methyltransferase, Streptomyces } \\
\text { puniciscabiei(WP 069778184.1); 78\%/93\% }\end{array}$ & NanM \\
\hline pak32 & 316 & NAD-dependent epimerase/dehydratase & $\begin{array}{c}\text { NAD }(\mathrm{P}) \text {-dependent oxidoreductase, Streptomyces } \\
\text { puniciscabiei(WP_099054848.1); 72\%/99\% }\end{array}$ & NanG4 \\
\hline pak33 & 434 & $\begin{array}{l}\text { Lipopolysaccharide biosynthesis protein } \\
\qquad \mathrm{RfbH}\end{array}$ & $\begin{array}{c}\text { Lipopolysaccharide biosynthesis protein RfbH, } \\
\text { Streptomyces sp. NBS 14/10(WP_089509387.1); } \\
86 \% / 100 \%\end{array}$ & NanG3 \\
\hline pak34 & 475 & NDP-hexose 2,3-dehydratase & $\begin{array}{c}\text { NDP-hexose 2,3-dehydratase, Streptomyces } \\
\text { oceani(WP_070197014.1); 68\%/96\% }\end{array}$ & - \\
\hline pak35 & 335 & Sugar 3-ketoreductase & $\begin{array}{l}\text { gfo/ldh/MocA family oxidoreductase, Streptomyces } \\
\text { sp. CB01201(WP_100574260.1) }\end{array}$ & LobD7 \\
\hline pak36 & 326 & dTDP-glucose 4,6-dehydratase & $\begin{array}{l}\text { dTDP-glucose 4,6-dehydratase, Streptomyces sp. } \\
\text { NRRL S-920(WP_030788407.1); 84\%/98\% }\end{array}$ & NanG2 \\
\hline pak37 & 302 & $\begin{array}{l}\text { Glucose-1-phosphate } \\
\text { adenylyl/thymidylyltransferase }\end{array}$ & $\begin{array}{c}\text { Glucose-1-phosphate thymidylyltransferase, } \\
\text { Streptomyces sparsoqenes(WP_065957879.1); } \\
85 \% / 100 \%\end{array}$ & NanG1 \\
\hline pak38 & 291 & O-methyltransferase & $\begin{array}{c}\text { Methyltransferase domain containing protein, } \\
\text { Streptomyces malaysiensis (WP_099015918.1); } \\
60 \% / 98 \%\end{array}$ & NigE \\
\hline pak39 & 231 & Transcriptional regulator & $\begin{array}{c}\text { TetR/AcrR family transcriptional regulator, } \\
\text { Streptomyces sp. CNZ279(WP_099877394.1); } \\
97 \% / 100 \%\end{array}$ & - \\
\hline pak40 & 314 & ABC transporter ATP-binding protein & $\begin{array}{c}\text { ABC transporter ATP-binding protein, Streptomyces } \\
\text { sp. CNZ279(WP_099877391.1); 98\%/100\% }\end{array}$ & - \\
\hline pak41 & 531 & $\mathrm{ABC}$ transporter permease & $\begin{array}{l}\text { ABC transporter permease, Streptomyces sp. } \\
\text { CNZ279(WP_099877388.1); 99\%/100\% }\end{array}$ & - \\
\hline
\end{tabular}

\footnotetext{
${ }^{\mathrm{a}}$ Size in units of amino acids (aa); ID/SI: identity/similarity.
} 
Table S2. The ${ }^{1} \mathrm{H}$ and ${ }^{13} \mathrm{C}$ NMR data for compounds 3 and $\mathbf{4}$ in $\mathrm{CDCl}_{3}$.

\begin{tabular}{|c|c|c|c|c|c|}
\hline \multirow{2}{*}{ Position } & \multicolumn{2}{|r|}{$\mathrm{K}-41 \mathrm{C}(\mathbf{3})$} & \multirow{2}{*}{ Position } & \multicolumn{2}{|r|}{ K-41D (4) } \\
\hline & $\delta_{\mathrm{C}}$ type & $\delta_{\mathrm{H}}$ mult. $(J$ in $\mathrm{Hz})$ & & $\delta_{\mathrm{C}}$ type & $\delta_{\mathrm{H}}$ mult. $(J$ in $\mathrm{Hz})$ \\
\hline 1 & $178.9, \mathrm{C}$ & & 1 & $178.9, \mathrm{C}$ & \\
\hline 2 & $71.9, \mathrm{CH}$ & 3.91, overlapped & 2 & $71.9, \mathrm{CH}$ & 3.89 , overlapped \\
\hline 3 & $99.2, \mathrm{C}$ & & 3 & $99.1, \mathrm{C}$ & \\
\hline 4 & $38.3, \mathrm{CH}$ & 2.19, overlapped & 4 & $38.3, \mathrm{CH}$ & 2.18 , overlapped \\
\hline 5 & 73.7, $\mathrm{CH}$ & 3.88 , overlapped & 5 & $73.8, \mathrm{CH}$ & 3.90 , overlapped \\
\hline 6 & $78.0, \mathrm{C}$ & & 6 & 77.9, C & \\
\hline 7 & $65.8, \mathrm{CH}$ & 3.92, overlapped & 7 & $65.7, \mathrm{CH}$ & 3.91, overlapped \\
\hline 8 & $32.8, \mathrm{CH}_{2}$ & $1.63,1.54$, overlapped & 8 & $32.8, \mathrm{CH}_{2}$ & $1.62,1.52$, overlapped \\
\hline 9 & $61.3, \mathrm{CH}$ & 3.99 , overlapped & 9 & $61.3, \mathrm{CH}$ & 3.98, overlapped \\
\hline 10 & $31.2, \mathrm{CH}_{2}$ & $1.16,2.11$, overlapped & 10 & $31.2, \mathrm{CH}_{2}$ & $2.10,1.15$, overlapped \\
\hline 11 & 79.9, $\mathrm{CH}$ & 3.38, overlapped & 11 & $79.8, \mathrm{CH}$ & 3.38 , overlapped \\
\hline 12 & $37.0, \mathrm{CH}$ & 1.82 , overlapped & 12 & $36.9, \mathrm{CH}$ & 1.81 , overlapped \\
\hline 13 & 107.0, C & & 13 & 107.0, C & \\
\hline 14 & $46.3, \mathrm{CH}$ & 2.13 , overlapped & 14 & $46.3, \mathrm{CH}$ & 2.13 , overlapped \\
\hline 15 & $94.8, \mathrm{CH}$ & $3.55, \mathrm{~d}(9.0)$ & 15 & $94.8, \mathrm{CH}$ & $3.53, \mathrm{~d}(9.5)$ \\
\hline 16 & $83.5, \mathrm{C}$ & & 16 & $83.5, \mathrm{C}$ & \\
\hline 17 & $83.8, \mathrm{CH}$ & $3.76, \mathrm{~d}(7.0)$ & 17 & $83.8, \mathrm{CH}$ & 3.75 , overlapped \\
\hline 18 & 25.7, $\mathrm{CH}_{2}$ & 1.96 , overlapped & 18 & 25.7, $\mathrm{CH}_{2}$ & $1.79,1.95$, overlapped \\
\hline 19 & $23.2, \mathrm{CH}_{2}$ & 1.78 , overlapped & 19 & $23.2, \mathrm{CH}_{2}$ & 1.77, overlapped \\
\hline 20 & 79.6, CH & $4.55, \mathrm{dd}(9.5,5.5)$ & 20 & $79.5, \mathrm{CH}$ & $4.52, \mathrm{~m}$ \\
\hline 21 & 79.5, CH & 3.96, overlapped & 21 & 77.4, $\mathrm{CH}$ & 3.83, overlapped \\
\hline 22 & $29.3, \mathrm{CH}_{2}$ & 1.47, overlapped; $2.03, \mathrm{~m}$ & 22 & $29.3, \mathrm{CH}_{2}$ & $1.97,1.25$, overlapped \\
\hline 23 & $24.3, \mathrm{CH}_{2}$ & $2.21,1.85$, overlapped & 23 & $24.3, \mathrm{CH}_{2}$ & 2.15 , overlapped \\
\hline 24 & $80.8, \mathrm{CH}$ & $4.35, \mathrm{~m}$ & 24 & $80.9, \mathrm{CH}$ & $4.37, \mathrm{~m}$ \\
\hline 25 & $73.8, \mathrm{CH}$ & 3.90 , overlapped & 25 & 74.4, CH & 3.28 , overlapped \\
\hline 26 & $40.5, \mathrm{CH}$ & 1.22 , overlapped & 26 & $39.2, \mathrm{CH}$ & 1.27 , overlapped \\
\hline 27 & $75.2, \mathrm{CH}$ & 3.31 , overlapped & 27 & 79.4, $\mathrm{CH}$ & 3.95 , overlapped \\
\hline 28 & $47.0, \mathrm{CH}$ & $1.36, \mathrm{~m}$ & 28 & $47.1, \mathrm{CH}$ & 1.48 , overlapped \\
\hline 29 & $98.4, \mathrm{C}$ & & 29 & $98.5, \mathrm{C}$ & \\
\hline 4-Me & $12.2, \mathrm{CH}_{3}$ & $1.11, \mathrm{~d}(6.5)$ & 4-Me & $12.2, \mathrm{CH}_{3}$ & $1.11, \mathrm{~d}(6.5)$ \\
\hline 6-Me & $11.5, \mathrm{CH}_{3}$ & $1.14, \mathrm{~s}$ & $6-\mathrm{Me}$ & $11.5, \mathrm{CH}_{3}$ & $1.13, \mathrm{~s}$ \\
\hline $12-\mathrm{Me}$ & $12.5, \mathrm{CH}_{3}$ & $1.00, \mathrm{~d}(7.0)$ & $12-\mathrm{Me}$ & $12.7, \mathrm{CH}_{3}$ & 0.99 , overlapped \\
\hline 14-Me & $11.7, \mathrm{CH}_{3}$ & $1.02, \mathrm{~d}(6.5)$ & 14-Me & $11.7, \mathrm{CH}_{3}$ & 1.02, overlapped \\
\hline 16-Me & $28.6, \mathrm{CH}_{3}$ & $1.61, \mathrm{~s}$ & 16-Me & 28.6, $\mathrm{CH}_{3}$ & $1.61, \mathrm{~s}$ \\
\hline $26-\mathrm{Me}$ & $13.3, \mathrm{CH}_{3}$ & $0.99, \mathrm{~d}(6.5)$ & $26-\mathrm{Me}$ & $13.5, \mathrm{CH}_{3}$ & 1.01 , overlapped \\
\hline 28-Me & $12.8, \mathrm{CH}_{3}$ & $1.08, \mathrm{~d}(7.0)$ & 28-Me & $12.9, \mathrm{CH}_{3}$ & 1.03 , overlapped \\
\hline 29-Me & $27.1, \mathrm{CH}_{3}$ & $1.28, \mathrm{~s}$ & 29-Me & $27.0, \mathrm{CH}_{3}$ & $1.28, \mathrm{~s}$ \\
\hline $6-\mathrm{OMe}$ & $50.6, \mathrm{CH}_{3}$ & $3.32, \mathrm{~s}$ & $6-\mathrm{OMe}$ & $50.6, \mathrm{CH}_{3}$ & $3.32, \mathrm{~s}$ \\
\hline 11-OMe & $59.3, \mathrm{CH}_{3}$ & $3.43, \mathrm{~s}$ & $11-\mathrm{OMe}$ & $59.4, \mathrm{CH}_{3}$ & $3.45, \mathrm{~s}$ \\
\hline 15-OMe & $60.4, \mathrm{CH}_{3}$ & $3.41, \mathrm{~s}$ & 15-OMe & $60.4, \mathrm{CH}_{3}$ & $3.41, \mathrm{~s}$ \\
\hline $1^{\prime}$ & & & $1^{\prime}$ & $102.8, \mathrm{CH}$ & $4.43, \mathrm{dd}(9.4,1.7)$ \\
\hline $2^{\prime}$ & & & $2^{\prime}$ & $30.6, \mathrm{CH}_{2}$ & 1.44, overlapped \\
\hline $3^{\prime}$ & & & $3^{\prime}$ & 27.4, $\mathrm{CH}_{2}$ & $2.19,1.31$, overlapped \\
\hline $4^{\prime}$ & & & $4^{\prime}$ & $80.5, \mathrm{CH}$ & $2.79, \mathrm{~m}$ \\
\hline 4'-OMe & & & 4'-OMe & $56.9, \mathrm{CH}_{3}$ & $3.34, \mathrm{~s}$ \\
\hline $5^{\prime}$ & & & $5^{\prime}$ & 74.6, CH & 3.28 , overlapped \\
\hline 5'-Me & & & 5'-Me & $18.4, \mathrm{CH}_{3}$ & $1.21, \mathrm{~d}(6.5)$ \\
\hline
\end{tabular}


Table S3. The ${ }^{1} \mathrm{H}$ and ${ }^{13} \mathrm{C}$ NMR data for compounds 5 and $\mathbf{6}$ in $\mathrm{CDCl}_{3}$.

\begin{tabular}{|c|c|c|c|c|c|}
\hline \multirow{2}{*}{ Position } & \multicolumn{2}{|r|}{$\mathrm{K}-41 \mathrm{E}(\mathbf{5})$} & \multirow{2}{*}{ Position } & \multicolumn{2}{|r|}{$\mathrm{K}-41 \mathrm{~B}(\mathbf{6})$} \\
\hline & $\delta_{\mathrm{C}}$ type & $\delta_{\mathrm{H}}$ mult. $(\mathrm{J}$ in $\mathrm{Hz})$ & & $\delta_{\mathrm{C}}$ type & $\delta_{\mathrm{H}}$ mult. $(J$ in Hz$)$ \\
\hline 1 & 179.0, C & & 1 & $178.9, \mathrm{C}$ & \\
\hline 2 & $71.9, \mathrm{CH}$ & 3.90, overlapped & 2 & $72.0, \mathrm{CH}$ & 3.88 , overlapped \\
\hline 3 & $99.3, \mathrm{C}$ & & 3 & $99.1, \mathrm{C}$ & \\
\hline 4 & $38.7, \mathrm{CH}$ & 2.13 , overlapped & 4 & $38.8, \mathrm{CH}$ & 2.14 , overlapped \\
\hline 5 & $88.6, \mathrm{CH}$ & $3.16, \mathrm{~d}(11.3)$ & 5 & $85.6, \mathrm{CH}$ & 3.34, overlapped \\
\hline 6 & $74.9, \mathrm{C}$ & & 6 & $78.5, \mathrm{C}$ & \\
\hline 7 & $68.8, \mathrm{CH}$ & 3.73 , overlapped & 7 & $66.8, \mathrm{CH}$ & $3.81, \mathrm{dd}(12.3,2.2)$ \\
\hline 8 & $32.2, \mathrm{CH}_{2}$ & 1.59 , overlapped & 8 & $32.5, \mathrm{CH}_{2}$ & $1.52,1.58$, overlapped \\
\hline 9 & $61.4, \mathrm{CH}$ & 3.98 , overlapped & 9 & $61.4, \mathrm{CH}$ & $3.96, \mathrm{t}(11.4)$ \\
\hline 10 & $31.1, \mathrm{CH}_{2}$ & $1.15,2.10$, overlapped & 10 & $31.2, \mathrm{CH}_{2}$ & 1.14 , overlapped; $2.09, \mathrm{~d}(14.1)$ \\
\hline 11 & $79.9, \mathrm{CH}$ & $3.37, \mathrm{t}(10.1)$ & 11 & $79.9, \mathrm{CH}$ & 3.36, overlapped \\
\hline 12 & $36.9, \mathrm{CH}$ & 1.80, overlapped & 12 & $27.1, \mathrm{CH}$ & 1.79 , overlapped \\
\hline 13 & 107.0, C & & 13 & $106.4, \mathrm{C}$ & \\
\hline 14 & $46.3, \mathrm{CH}$ & 2.12 , overlapped & 14 & $46.3, \mathrm{CH}$ & 2.14 , overlapped \\
\hline 15 & $94.8, \mathrm{CH}$ & $3.53, \mathrm{~d}(9.2)$ & 15 & $93.1, \mathrm{CH}$ & $3.71, \mathrm{~d}(9.5)$ \\
\hline 16 & $83.5, \mathrm{C}$ & & 16 & $84.0, \mathrm{C}$ & \\
\hline 17 & $83.8, \mathrm{CH}$ & 3.74, overlapped & 17 & $84.3, \mathrm{CH}$ & 3.93, overlapped \\
\hline 18 & $25.7, \mathrm{CH}_{2}$ & $1.96,1.77$, overlapped & 18 & $25.4, \mathrm{CH}_{2}$ & 1.92, overlapped \\
\hline 19 & $23.2, \mathrm{CH}_{2}$ & 1.76, overlapped & 19 & $23.2, \mathrm{CH}_{2}$ & 1.76, overlapped \\
\hline 20 & $79.5, \mathrm{CH}$ & 4.52, ddd $(10.9,5.2,1.5)$ & 20 & $79.5, \mathrm{CH}$ & 4.52, ddd $(10.9,5.1,1.4)$ \\
\hline 21 & $79.4, \mathrm{CH}$ & 3.94, overlapped & 21 & $79.3, \mathrm{CH}$ & 3.92, overlapped \\
\hline 22 & $29.3, \mathrm{CH}_{2}$ & $1.43,1.96$, overlapped & 22 & $29.4, \mathrm{CH}_{2}$ & $1.97,1.43$, overlapped \\
\hline 23 & $24.3, \mathrm{CH}_{2}$ & $2.15,1.84$, overlapped & 23 & $24.4, \mathrm{CH}_{2}$ & $1.85,2.12$, overlapped \\
\hline 24 & $80.9, \mathrm{CH}$ & $4.37, \mathrm{~m}$ & 24 & $80.8, \mathrm{CH}$ & $4.36, \mathrm{~m}$ \\
\hline 25 & $74.4, \mathrm{CH}$ & 3.91, overlapped & 25 & $74.4, \mathrm{CH}$ & $3.90, \mathrm{dd}(10.6,2.7)$ \\
\hline 26 & $39.2, \mathrm{CH}$ & 1.27 , overlapped & 26 & $39.2, \mathrm{CH}$ & 1.26, overlapped \\
\hline 27 & $83.1, \mathrm{CH}$ & 3.38, overlapped & 27 & $83.1, \mathrm{CH}$ & 3.37 , overlapped \\
\hline 28 & $47.1, \mathrm{CH}$ & 1.47 , overlapped & 28 & $47.1, \mathrm{CH}$ & 1.47 , overlapped \\
\hline 29 & $98.5, \mathrm{C}$ & & 29 & $98.5, \mathrm{C}$ & \\
\hline 4-Me & $12.4, \mathrm{CH}_{3}$ & $1.08, \mathrm{~d}(6.7)$ & 4-Me & $12.3, \mathrm{CH}_{3}$ & $1.07, \mathrm{~d}(6.7)$ \\
\hline 6-Me & $14.7, \mathrm{CH}_{3}$ & $1.14, \mathrm{~s}$ & 6-Me & $11.2, \mathrm{CH}_{3}$ & $1.16, \mathrm{~s}$ \\
\hline $12-\mathrm{Me}$ & $12.9, \mathrm{CH}_{3}$ & $0.98, \mathrm{~d}(7.1)$ & $12-\mathrm{Me}$ & $12.8, \mathrm{CH}_{3}$ & $0.99, \mathrm{~d}(7.1)$ \\
\hline 14-Me & $11.6, \mathrm{CH}_{3}$ & $1.01, \mathrm{~d}(6.7)$ & 14-Me & $11.2, \mathrm{CH}_{3}$ & $0.96, \mathrm{~d}(6.7)$ \\
\hline 16-Me & $28.6, \mathrm{CH}_{3}$ & $1.61, \mathrm{~s}$ & 16-Me & $26.0, \mathrm{CH}_{3}$ & $1.59, \mathrm{~s}$ \\
\hline 26-Me & $13.5, \mathrm{CH}_{3}$ & $1.01, \mathrm{~d}(6.7)$ & 26-Me & $13.5, \mathrm{CH}_{3}$ & $1.01, \mathrm{~d}(6.4)$ \\
\hline 28-Me & $12.8, \mathrm{CH}_{3}$ & $1.03, \mathrm{~d}(6.7)$ & 28-Me & $12.9, \mathrm{CH}_{3}$ & $1.03, \mathrm{~d}(6.8)$ \\
\hline 29-Me & $27.0, \mathrm{CH}_{3}$ & $1.28, \mathrm{~s}$ & 29-Me & $26.9, \mathrm{CH}_{3}$ & $1.28, \mathrm{~s}$ \\
\hline $5-\mathrm{OMe}$ & $62.5, \mathrm{CH}_{3}$ & $3.59, \mathrm{~s}$ & 5-OMe & $61.2, \mathrm{CH}_{3}$ & $3.55, \mathrm{~s}$ \\
\hline 6-OMe & & & 6-OMe & $51.0, \mathrm{CH}_{3}$ & $3.36, \mathrm{~s}$ \\
\hline 11-OMe & $59.4, \mathrm{CH}_{3}$ & $3.46, \mathrm{~s}$ & 11-OMe & $59.4, \mathrm{CH}_{3}$ & $3.45, \mathrm{~s}$ \\
\hline 15-OMe & $60.4, \mathrm{CH}_{3}$ & $3.41, \mathrm{~s}$ & $15-\mathrm{OMe}$ & & \\
\hline $1^{\prime}$ & $102.8, \mathrm{CH}$ & $4.43, \mathrm{dd}(9.5,1.8)$ & $1^{\prime}$ & $102.8, \mathrm{CH}$ & $4.44, \mathrm{dd}(9.5,1.7)$ \\
\hline $2^{\prime}$ & $30.6, \mathrm{CH}_{2}$ & $1.48,1.97$, overlapped & $2^{\prime}$ & $30.6, \mathrm{CH}_{2}$ & $1.90,1.47$, overlapped \\
\hline $3^{\prime}$ & $27.5, \mathrm{CH}_{2}$ & 2.19, overlapped & $3^{\prime}$ & $27.5, \mathrm{CH}_{2}$ & 2.20, overlapped \\
\hline 4' & $80.5, \mathrm{CH}$ & $2.79, \mathrm{~m}$ & 4' & $80.5, \mathrm{CH}$ & $2.78, \mathrm{~m}$ \\
\hline 4'-OMe & $57.0, \mathrm{CH}_{3}$ & $3.35, \mathrm{~s}$ & 4'-OMe & $57.0, \mathrm{CH}_{3}$ & $3.34, \mathrm{~s}$ \\
\hline $5^{\prime}$ & $74.6, \mathrm{CH}$ & $3.28, \mathrm{~m}$ & $5^{\prime}$ & $74.6, \mathrm{CH}$ & $3.28, \mathrm{~m}$ \\
\hline 5'-Me & $18.4, \mathrm{CH}_{3}$ & $1.22, \mathrm{~d}(6.1)$ & 5'-Me & $18.4, \mathrm{CH}_{3}$ & $1.22, \mathrm{~d}(6.1)$ \\
\hline & & & 1" & 103.6, CH & $4.34, \mathrm{dd}(9.5,1.8)$ \\
\hline & & & $2^{\prime \prime}$ & $30.7, \mathrm{CH}_{2}$ & $1.90,1.45$, overlapped \\
\hline & & & 3" & $27.2, \mathrm{CH}_{2}$ & 2.20, overlapped \\
\hline & & & 4" & $80.2, \mathrm{CH}$ & $2.78, \mathrm{~m}$ \\
\hline & & & 4"-OMe & $57.1, \mathrm{CH}_{3}$ & $3.34, \mathrm{~s}$ \\
\hline & & & $5^{\prime \prime}$ & $74.8, \mathrm{CH}$ & $3.28, \mathrm{~m}$ \\
\hline & & & 5"-Me & $18.4, \mathrm{CH}_{3}$ & $1.23, \mathrm{~d}(6.1)$ \\
\hline
\end{tabular}


Table S4. The ${ }^{1} \mathrm{H}$ and ${ }^{13} \mathrm{C}$ NMR data for compounds 7 and 8 in $\mathrm{CDCl}_{3}$.

\begin{tabular}{|c|c|c|c|c|c|}
\hline \multirow{2}{*}{ Position } & \multicolumn{2}{|r|}{$\mathrm{K}-41 \mathrm{Bm}(7)$} & \multirow{2}{*}{ Position } & \multicolumn{2}{|r|}{$\mathrm{K}-41 \mathrm{~F}(\mathbf{8})$} \\
\hline & $\delta_{\mathrm{C}}$ type & $\delta_{\mathrm{H}}$ mult. $(J$ in $\mathrm{Hz})$ & & $\delta_{\mathrm{C}}$ type & $\delta_{\mathrm{H}}$ mult. $(J$ in $\mathrm{Hz})$ \\
\hline 1 & $178.5, \mathrm{C}$ & & 1 & 179.0, C & \\
\hline 2 & $71.2, \mathrm{CH}$ & $3.85, \mathrm{~s}$ & 2 & $72.0, \mathrm{CH}$ & $3.89, \mathrm{~d}(5.0)$ \\
\hline 3 & $99.2, \mathrm{C}$ & & 3 & $99.1, \mathrm{C}$ & \\
\hline 4 & $38.7, \mathrm{CH}$ & 2.15 , overlapped & 4 & $38.7, \mathrm{CH}$ & 2.15 , overlapped \\
\hline 5 & $85.4, \mathrm{CH}$ & 3.34 , overlapped & 5 & 85.6, CH & $3.33, \mathrm{~d}(11.0)$ \\
\hline 6 & $78.5, \mathrm{C}$ & & 6 & $78.4, \mathrm{C}$ & \\
\hline 7 & $66.5, \mathrm{CH}$ & $3.84, \mathrm{dd}(12.5,2.0)$ & 7 & $66.9, \mathrm{CH}$ & 3.80 , overlapped \\
\hline 8 & $32.3, \mathrm{CH}_{2}$ & $1.56,1.50$, overlapped & 8 & $32.5, \mathrm{CH}_{2}$ & 1.53 , overlapped \\
\hline 9 & $61.9, \mathrm{CH}$ & $3.90, \mathrm{t}(11.6)$ & 9 & $61.5, \mathrm{CH}$ & 3.95 , overlapped \\
\hline 10 & $31.2, \mathrm{CH}_{2}$ & $2.12,1.13$, overlapped & 10 & $31.1, \mathrm{CH}_{2}$ & $1.15,2.09$, overlapped \\
\hline 11 & $79.5, \mathrm{CH}$ & 3.34, overlapped & 11 & $79.9, \mathrm{CH}$ & 3.37, overlapped \\
\hline 12 & $37.2, \mathrm{CH}$ & 1.77 , overlapped & 12 & $36.9, \mathrm{CH}$ & 1.79 , overlapped \\
\hline 13 & 106.6, C & & 13 & 107.0, C & \\
\hline 14 & 46.6, CH & 2.17 , overlapped & 14 & $46.3, \mathrm{CH}$ & 2.12 , overlapped \\
\hline 15 & 93.1, CH & $3.69, \mathrm{~d}(8.4)$ & 15 & $94.7, \mathrm{CH}$ & $3.53, \mathrm{~d}(9.3)$ \\
\hline 16 & $84.8, \mathrm{C}$ & & 16 & $83.5, \mathrm{C}$ & \\
\hline 17 & $83.7, \mathrm{CH}$ & $4.00, \mathrm{t}(7.7)$ & 17 & $83.8, \mathrm{CH}$ & $3.74, \mathrm{t}(1.3)$ \\
\hline 18 & $25.9, \mathrm{CH}_{2}$ & 2.15 , overlapped & 18 & $25.6, \mathrm{CH}_{2}$ & 1.94, 1.76, overlapped \\
\hline 19 & $23.3, \mathrm{CH}_{2}$ & 1.19, overlapped & 19 & $23.2, \mathrm{CH}_{2}$ & 1.77, overlapped \\
\hline 20 & $81.4, \mathrm{CH}$ & 3.73 , ddd $(10.3,5.8,2.2)$ & 20 & $79.5, \mathrm{CH}$ & 4.52 , ddd $(10.9,5.2,1.6)$ \\
\hline 21 & $78.7, \mathrm{CH}$ & 4.86, dt $(9.0,3.4)$ & 21 & $79.4, \mathrm{CH}$ & 3.94 , overlapped \\
\hline 22 & $28.5, \mathrm{CH}_{2}$ & 1.55 , dd $(12.4,2.8) ; 1.30$, overlapped & 22 & 29.3, $\mathrm{CH}_{2}$ & $1.96,1.43$, overlapped \\
\hline 23 & 24.6, $\mathrm{CH}_{2}$ & 2.01, t (11.4); 1.77, overlapped & 23 & $24.3, \mathrm{CH}_{2}$ & $1.82,2.14$, overlapped \\
\hline 24 & $81.2, \mathrm{CH}$ & $4.43, \mathrm{~d}(8.4)$ & 24 & $80.8, \mathrm{CH}$ & $4.37, \mathrm{~m}$ \\
\hline 25 & 78.1, CH & $3.59, \mathrm{~d}(10.9)$ & 25 & $74.3, \mathrm{CH}$ & 3.91, dd $(10.6,2.7)$ \\
\hline 26 & $38.8, \mathrm{CH}$ & 2.15 , overlapped & 26 & $39.2, \mathrm{CH}$ & 1.28 , overlapped \\
\hline 27 & $82.8, \mathrm{CH}$ & 3.35 , overlapped & 27 & $83.0, \mathrm{CH}$ & 3.46 , overlapped \\
\hline 28 & $47.7, \mathrm{CH}$ & 1.49 , overlapped & 28 & $47.2, \mathrm{CH}$ & 1.51 , overlapped \\
\hline 29 & $100.9, \mathrm{C}$ & & 29 & $98.5, \mathrm{C}$ & \\
\hline 4-Me & $12.2, \mathrm{CH}_{3}$ & $1.06, \mathrm{~d}(6.7)$ & 4-Me & $12.2, \mathrm{CH}_{3}$ & $1.06, \mathrm{~d}(6.7)$ \\
\hline 6-Me & $11.3, \mathrm{CH}_{3}$ & $1.15, \mathrm{~s}$ & 6-Me & $11.1, \mathrm{CH}_{3}$ & $1.15, \mathrm{~s}$ \\
\hline 12-Me & $12.9, \mathrm{CH}_{3}$ & $0.97, \mathrm{~d}(7.6)$ & 12-Me & $12.8, \mathrm{CH}_{3}$ & $0.98, \mathrm{~d}(5.5)$ \\
\hline 14-Me & $11.3, \mathrm{CH}_{3}$ & $0.96, \mathrm{~d}(3.2)$ & 14-Me & $11.7, \mathrm{CH}_{3}$ & $1.00, \mathrm{~d}(6.8)$ \\
\hline $16-\mathrm{Me}$ & $25.9, \mathrm{CH}_{3}$ & $1.58, \mathrm{~s}$ & 16-Me & $28.6, \mathrm{CH}_{3}$ & $1.60, \mathrm{~s}$ \\
\hline 26-Me & 13.6, $\mathrm{CH}_{3}$ & $1.00, \mathrm{~d}(6.4)$ & 26-Me & $13.5, \mathrm{CH}_{3}$ & $0.99, \mathrm{~d}(6.4)$ \\
\hline 28-Me & $12.6, \mathrm{CH}_{3}$ & $0.95, \mathrm{~d}(2.9)$ & 28-Me & $13.0, \mathrm{CH}_{3}$ & $1.06, \mathrm{~d}(6.7)$ \\
\hline $29-\mathrm{Me}$ & $22.1, \mathrm{CH}_{3}$ & $1.20, \mathrm{~s}$ & $29-\mathrm{Me}$ & $26.9, \mathrm{CH}_{3}$ & $1.29, \mathrm{~s}$ \\
\hline 5-OMe & $61.1, \mathrm{CH}_{3}$ & $3.54, \mathrm{~s}$ & 5-OMe & $61.2, \mathrm{CH}_{3}$ & $3.54, \mathrm{~s}$ \\
\hline 6-OMe & $50.8, \mathrm{CH}_{3}$ & $3.34, \mathrm{~s}$ & 6-OMe & $51.0, \mathrm{CH}_{3}$ & $3.36, \mathrm{~s}$ \\
\hline 11-OMe & $59.0, \mathrm{CH}_{3}$ & $3.40, \mathrm{~s}$ & 11-OMe & $59.3, \mathrm{CH}_{3}$ & $3.44, \mathrm{~s}$ \\
\hline 15-OMe & & & $15-\mathrm{OMe}$ & $60.4, \mathrm{CH}_{3}$ & $3.40, \mathrm{~s}$ \\
\hline 29-OMe & $48.6, \mathrm{CH}_{3}$ & $3.10, \mathrm{~s}$ & 29-OMe & & \\
\hline 1' & $102.9, \mathrm{CH}$ & $4.39, \mathrm{dd}(9.5,1.5)$ & $1^{\prime}$ & $100.9, \mathrm{CH}$ & 4.94, dd $(8.1,2.8)$ \\
\hline $2^{\prime}$ & $30.6, \mathrm{CH}_{2}$ & $1.94,1.45$, overlapped & $2^{\prime}$ & $30.9, \mathrm{CH}_{2}$ & $2.28,1.54$, overlapped \\
\hline $3^{\prime}$ & $27.4, \mathrm{CH}_{2}$ & $2.26, \mathrm{~m} ; 1.17$, overlapped & $3^{\prime}$ & $35.5, \mathrm{CH}_{2}$ & $2.59, \mathrm{~m} ; 2.41, \mathrm{~m}$ \\
\hline 4' & $80.4, \mathrm{CH}$ & 2.76, overlapped & 4' & 208.7, C & \\
\hline 4'-OMe & $56.9, \mathrm{CH}_{3}$ & $3.32, \mathrm{~s}$ & 4'-OMe & & \\
\hline $5^{\prime}$ & $74.8, \mathrm{CH}$ & 3.26 , overlapped & $5^{\prime}$ & $76.0, \mathrm{CH}$ & $3.99, \mathrm{q}(6.4)$ \\
\hline 5'-Me & $18.4, \mathrm{CH}_{3}$ & $1.21, \mathrm{~d}(5.6)$ & 5'-Me & $15.3, \mathrm{CH}_{3}$ & $1.27, \mathrm{~d}(6.6)$ \\
\hline 1" & $103.5, \mathrm{CH}$ & $4.32, \mathrm{dd}(9.3,1.5)$ & & & \\
\hline $2^{\prime \prime}$ & $30.6, \mathrm{CH}_{2}$ & $1.89,1.45$, overlapped & & & \\
\hline 3" & 27.1, $\mathrm{CH}_{2}$ & $2.18,1.17$, overlapped & & & \\
\hline 4" & $80.2, \mathrm{CH}$ & 2.76, overlapped & & & \\
\hline 4"-OMe & $57.1, \mathrm{CH}_{3}$ & $3.32, \mathrm{~s}$ & & & \\
\hline $5 "$ & 74.6, CH & 3.26, overlapped & & & \\
\hline 5"-Me & $18.4, \mathrm{CH}_{3}$ & $1.21, \mathrm{~d}(5.6)$ & & & \\
\hline
\end{tabular}


Table S5. The ${ }^{1} \mathrm{H}$ and ${ }^{13} \mathrm{C}$ NMR data for compounds 9 and $\mathbf{1 0}$ in $\mathrm{CDCl}_{3}$.

\begin{tabular}{|c|c|c|c|c|c|}
\hline \multirow{2}{*}{ Position } & \multicolumn{2}{|r|}{ K-41G (9) } & \multirow{2}{*}{ Position } & \multicolumn{2}{|r|}{$\mathrm{K}-41 \mathrm{Gm}(\mathbf{1 0})$} \\
\hline & $\delta_{\mathrm{C}}$ type & $\delta_{\mathrm{H}}$ mult. $(J$ in $\mathrm{Hz})$ & & $\delta_{\mathrm{C}}$ type & $\delta_{\mathrm{H}}$ mult. $(J$ in Hz) \\
\hline 1 & $178.9, \mathrm{C}$ & & 1 & $178.6, \mathrm{C}$ & \\
\hline 2 & $71.9, \mathrm{CH}$ & 3.89 , overlapped & 2 & $71.3, \mathrm{CH}$ & $3.86, \mathrm{~d}(3.2)$ \\
\hline 3 & $99.1, \mathrm{C}$ & & 3 & $99.2, \mathrm{C}$ & \\
\hline 4 & $38.7, \mathrm{CH}$ & 2.14 , overlapped & 4 & $38.7, \mathrm{CH}$ & 2.15 , overlapped \\
\hline 5 & $85.6, \mathrm{CH}$ & $3.33, \mathrm{~d}(11.0)$ & 5 & $85.6, \mathrm{CH}$ & 3.33, overlapped \\
\hline 6 & 78.4, C & & 6 & $78.4, \mathrm{C}$ & \\
\hline 7 & $66.8, \mathrm{CH}$ & 3.80 , overlapped & 7 & $66.6, \mathrm{CH}$ & 3.83 , overlapped \\
\hline 8 & $32.4, \mathrm{CH}_{2}$ & 1.53 , overlapped & 8 & $32.4, \mathrm{CH}_{2}$ & 1.54 , overlapped \\
\hline 9 & $61.5, \mathrm{CH}$ & 3.94 , overlapped & 9 & $61.9, \mathrm{CH}$ & 3.90 , overlapped \\
\hline 10 & $31.1, \mathrm{CH}_{2}$ & $2.08,1.14$, overlapped & 10 & $31.1, \mathrm{CH}_{2}$ & $1.15,2.13$, overlapped \\
\hline 11 & $79.8, \mathrm{CH}$ & 3.36, overlapped & 11 & $79.5, \mathrm{CH}$ & 3.36, overlapped \\
\hline 12 & $36.9, \mathrm{CH}$ & 1.79 , overlapped & 12 & $37.1, \mathrm{CH}$ & 1.79 , overlapped \\
\hline 13 & 107.0, C & & 13 & 107.2, C & \\
\hline 14 & $46.3, \mathrm{CH}$ & 2.11, overlapped & 14 & $46.7, \mathrm{CH}$ & 2.16 , overlapped \\
\hline 15 & 94.7, $\mathrm{CH}$ & $3.52, \mathrm{~d}(9.3)$ & 15 & $95.0, \mathrm{CH}$ & $3.51, \mathrm{~d}(9.1)$ \\
\hline 16 & $83.5, \mathrm{C}$ & & 16 & $84.3, \mathrm{C}$ & \\
\hline 17 & $83.8, \mathrm{CH}$ & $3.74, \mathrm{t}(8.2)$ & 17 & $83.2, \mathrm{CH}$ & 3.82, overlapped \\
\hline 18 & $25.6, \mathrm{CH}_{2}$ & $1.91,1.76$, overlapped & 18 & 26.1, $\mathrm{CH}_{2}$ & $1.84,1.95$, overlapped \\
\hline 19 & $23.2, \mathrm{CH}_{2}$ & 1.74, overlapped & 19 & 23.3, $\mathrm{CH}_{2}$ & $1.52,1.75$, overlapped \\
\hline 20 & $79.5, \mathrm{CH}$ & $4.53, \operatorname{ddd}(10.7,5.3,1.0)$ & 20 & $78.7, \mathrm{CH}$ & 4.53, ddd $(10.9,5.3,1.6)$ \\
\hline 21 & $79.4, \mathrm{CH}$ & 3.93, overlapped & 21 & $77.4, \mathrm{CH}$ & $3.58, \mathrm{dd}(10.9,1.0)$ \\
\hline 22 & $29.3, \mathrm{CH}_{2}$ & $1.98,1.43$, overlapped & 22 & $28.5, \mathrm{CH}_{2}$ & 2.30, m; 1.50, overlapped \\
\hline 23 & $24.3, \mathrm{CH}_{2}$ & $2.18,1.83$, overlapped & 23 & $24.8, \mathrm{CH}_{2}$ & 1.80, overlapped; $2.06, \mathrm{t}(11.4)$ \\
\hline 24 & $80.8, \mathrm{CH}$ & $4.33, \mathrm{~m}$ & 24 & $81.4, \mathrm{CH}$ & $4.41, \mathrm{~d}(8.4)$ \\
\hline 25 & $73.7, \mathrm{CH}$ & 3.88 , overlapped & 25 & $74.7, \mathrm{CH}$ & $3.30, \mathrm{t}(9.9)$ \\
\hline 26 & $40.4, \mathrm{CH}$ & $1.20, \mathrm{~m}$ & 26 & $39.8, \mathrm{CH}$ & 1.24 , overlapped \\
\hline 27 & $75.1, \mathrm{CH}$ & $3.29, \mathrm{t}(9.9)$ & 27 & $81.5, \mathrm{CH}$ & $3.74, \mathrm{~m}$ \\
\hline 28 & $47.0, \mathrm{CH}$ & 1.34 , overlapped & 28 & $47.6, \mathrm{CH}$ & $1.39, \mathrm{~m}$ \\
\hline 29 & $98.3, \mathrm{C}$ & & 29 & $100.8, \mathrm{C}$ & \\
\hline 4-Me & $12.2, \mathrm{CH}_{3}$ & $1.06, \mathrm{~d}(2.9)$ & 4-Me & $12.2, \mathrm{CH}_{3}$ & $1.07, \mathrm{~d}(6.7)$ \\
\hline 6-Me & $11.1, \mathrm{CH}_{3}$ & $1.14, \mathrm{~s}$ & 6-Me & $11.2, \mathrm{CH}_{3}$ & $1.15, \mathrm{~s}$ \\
\hline 12-Me & $12.5, \mathrm{CH}_{3}$ & $0.98, \mathrm{~d}(7.1)$ & 12-Me & $12.2, \mathrm{CH}_{3}$ & $0.98, \mathrm{~d}(4.4)$ \\
\hline 14-Me & $11.6, \mathrm{CH}_{3}$ & $1.01, \mathrm{~d}(6.7)$ & 14-Me & $11.8, \mathrm{CH}_{3}$ & $1.03, \mathrm{~d}(6.8)$ \\
\hline $16-\mathrm{Me}$ & $28.6, \mathrm{CH}_{3}$ & $1.60, \mathrm{~s}$ & $16-\mathrm{Me}$ & $28.5, \mathrm{CH}_{3}$ & $1.60, \mathrm{~s}$ \\
\hline 26-Me & $13.3, \mathrm{CH}_{3}$ & $0.96, d(6.4)$ & 26-Me & $13.5, \mathrm{CH}_{3}$ & $0.97, \mathrm{~d}(3.7)$ \\
\hline 28-Me & $12.8, \mathrm{CH}_{3}$ & $1.07, \mathrm{~d}(2.9)$ & 28-Me & $12.9, \mathrm{CH}_{3}$ & $1.01, \mathrm{~d}(6.7)$ \\
\hline 29-Me & $27.0, \mathrm{CH}_{3}$ & $1.27, \mathrm{~s}$ & 29-Me & $22.1, \mathrm{CH}_{3}$ & $1.23, \mathrm{~s}$ \\
\hline $5-\mathrm{OMe}$ & $61.2, \mathrm{CH}_{3}$ & $3.54, \mathrm{~s}$ & $5-\mathrm{OMe}$ & $61.2, \mathrm{CH}_{3}$ & $3.55, \mathrm{~s}$ \\
\hline 6-OMe & $50.9, \mathrm{CH}_{3}$ & $3.35, \mathrm{~s}$ & 6-OMe & $50.8, \mathrm{CH}_{3}$ & $3.35, \mathrm{~s}$ \\
\hline 11-OMe & $59.2, \mathrm{CH}_{3}$ & $3.42, \mathrm{~s}$ & 11-OMe & $58.9, \mathrm{CH}_{3}$ & $3.39, \mathrm{~s}$ \\
\hline $15-\mathrm{OMe}$ & $60.4, \mathrm{CH}_{3}$ & $3.40, \mathrm{~s}$ & 15-OMe & $60.3, \mathrm{CH}_{3}$ & $3.40, \mathrm{~s}$ \\
\hline 29-OMe & & & 29-OMe & $48.6, \mathrm{CH}_{3}$ & $3.10, \mathrm{~s}$ \\
\hline
\end{tabular}


Table S6. The ${ }^{1} \mathrm{H}$ and ${ }^{13} \mathrm{C}$ NMR data for compound 11 in $\mathrm{CDCl}_{3}$.

\begin{tabular}{|c|c|c|}
\hline \multirow{2}{*}{ Position } & \multicolumn{2}{|c|}{ K-41H (11) } \\
\hline & $\delta_{\mathrm{C}}$ type & $\delta_{\mathrm{H}}$ mult. $(J$ in $\mathrm{Hz})$ \\
\hline 1 & $179.3, \mathrm{C}$ & \\
\hline 2 & $72.2, \mathrm{CH}$ & $3.89, \mathrm{~d}(5.9)$ \\
\hline 3 & $99.1, \mathrm{C}$ & \\
\hline 4 & $38.7, \mathrm{CH}$ & 2.12, overlapped \\
\hline 5 & $85.4, \mathrm{CH}$ & 3.36 , overlapped \\
\hline 6 & $78.6, \mathrm{C}$ & \\
\hline 7 & $66.4, \mathrm{CH}$ & $3.83, \mathrm{dd}(12.3,3.5)$ \\
\hline 8 & $36.7, \mathrm{CH}_{2}$ & 1.30, overlapped \\
\hline 9 & $61.3, \mathrm{CH}$ & $4.19, \operatorname{td}(11.4,3.1)$ \\
\hline 10 & $31.7, \mathrm{CH}_{2}$ & $1.68, \operatorname{td}(12.5,3.6)$ \\
\hline 11 & $68.6, \mathrm{CH}$ & 4.02, overlapped \\
\hline 12 & $37.0, \mathrm{CH}$ & 1.74 , overlapped \\
\hline 13 & 107.2, C & \\
\hline 14 & $45.9, \mathrm{CH}$ & 2.11, overlapped \\
\hline 15 & $94.4, \mathrm{CH}$ & $3.59, \mathrm{~d}(9.6)$ \\
\hline 16 & $82.0, \mathrm{C}$ & \\
\hline 17 & $84.5, \mathrm{CH}$ & $3.69, \mathrm{dd}(10.2,5.3)$ \\
\hline 18 & $26.2, \mathrm{CH}_{2}$ & $1.77,1.98$, overlapped \\
\hline 19 & 24.1, $\mathrm{CH}_{2}$ & 1.49, overlapped \\
\hline 20 & $78.9, \mathrm{CH}$ & $4.03, \operatorname{td}(7.6,1.2)$ \\
\hline 21 & $80.4, \mathrm{CH}$ & $4.31, \mathrm{dd}(11.1,4.9)$ \\
\hline 22 & $29.1, \mathrm{CH}_{2}$ & $1.98,1.49$, overlapped \\
\hline 23 & 24.6, $\mathrm{CH}_{2}$ & $2.15,1.84$, overlapped \\
\hline 24 & $80.8, \mathrm{CH}$ & $4.27, \mathrm{~m}$ \\
\hline 25 & $73.5, \mathrm{CH}$ & $4.00, \mathrm{dd}(10.7,2.2)$ \\
\hline 26 & $39.3, \mathrm{CH}$ & 1.23 , overlapped \\
\hline 27 & $81.8, \mathrm{CH}$ & $3.41, \mathrm{t}(10.2)$ \\
\hline 28 & $47.5, \mathrm{CH}$ & 1.47 , overlapped \\
\hline 29 & $98.7, \mathrm{C}$ & \\
\hline 4-Me & $12.1, \mathrm{CH}_{3}$ & $1.06, \mathrm{~d}(6.7)$ \\
\hline 6-Me & $11.2, \mathrm{CH}_{3}$ & $1.16, \mathrm{~s}$ \\
\hline 12-Me & $12.8, \mathrm{CH}_{3}$ & $1.00, \mathrm{~d}(6.7)$ \\
\hline 14-Me & $11.3, \mathrm{CH}_{3}$ & $1.00, \mathrm{~d}(6.7)$ \\
\hline $16-\mathrm{Me}$ & $28.4, \mathrm{CH}_{3}$ & $1.51, \mathrm{~s}$ \\
\hline 26-Me & $13.5, \mathrm{CH}_{3}$ & $1.00, \mathrm{~d}(6.7)$ \\
\hline 28-Me & $13.0, \mathrm{CH}_{3}$ & $1.04, \mathrm{~d}(6.7)$ \\
\hline 29-Me & $26.7, \mathrm{CH}_{3}$ & $1.31, \mathrm{~s}$ \\
\hline 5-OMe & $61.3, \mathrm{CH}_{3}$ & $3.55, \mathrm{~s}$ \\
\hline 6-OMe & $51.0, \mathrm{CH}_{3}$ & $3.35, \mathrm{~s}$ \\
\hline 15-OMe & $60.5, \mathrm{CH}_{3}$ & $3.42, \mathrm{~s}$ \\
\hline $1^{\prime}$ & 102.7, $\mathrm{CH}$ & 4.43, dd $(9.4,1.3)$ \\
\hline $2^{\prime}$ & $30.5, \mathrm{CH}_{2}$ & $1.97,1.45$, overlapped \\
\hline $3^{\prime}$ & $27.5, \mathrm{CH}_{2}$ & 2.20, 1.29, overlapped \\
\hline $4^{\prime}$ & $80.6, \mathrm{CH}$ & $2.79, \mathrm{~m}$ \\
\hline 4'-OMe & $57.0, \mathrm{CH}_{3}$ & $3.34, \mathrm{~s}$ \\
\hline $5^{\prime}$ & $74.6, \mathrm{CH}$ & $3.24, \mathrm{~m}$ \\
\hline 5'-Me & $18.4, \mathrm{CH}_{3}$ & $1.23, \mathrm{~d}(6.1)$ \\
\hline
\end{tabular}


Table S7. Antibacterial activities determined for compounds 3-11. ${ }^{\mathrm{a}}$

\begin{tabular}{|c|c|c|c|c|c|c|c|c|}
\hline \multirow{2}{*}{ Compound } & \multicolumn{8}{|c|}{ MIC values $(\mu \mathrm{g} / \mathrm{mL})$} \\
\hline & ML & Ljh13 & SA1 & SA2 & SA3 & SA4 & BS & MRSA \\
\hline $\mathrm{K}-41 \mathrm{C}(\mathbf{3})$ & $>64$ & $>64$ & $>64$ & $>64$ & $>64$ & $>64$ & $>64$ & $>64$ \\
\hline $\mathrm{K}-41 \mathrm{D}(\mathbf{4})$ & 64 & 32 & 32 & 16 & 16 & $>64$ & 64 & 64 \\
\hline $\mathrm{K}-41 \mathrm{E}(\mathbf{5})$ & 32 & 8 & 16 & 16 & 32 & 32 & 16 & 32 \\
\hline $\mathrm{K}-41 \mathrm{~B}(\mathbf{6})$ & 8 & 2 & 4 & 8 & 8 & 8 & 4 & 4 \\
\hline $\mathrm{K}-41 \mathrm{Bm}(7)$ & 8 & 4 & 4 & 4 & 8 & 16 & 4 & 8 \\
\hline $\mathrm{K}-41 \mathrm{~F}(\mathbf{8})$ & 16 & 8 & 16 & 16 & 16 & 32 & 16 & 16 \\
\hline $\mathrm{K}-41 \mathrm{G}(\mathbf{9})$ & $>64$ & 64 & $>64$ & 64 & $>64$ & $>64$ & 64 & $>64$ \\
\hline $\mathrm{K}-41 \mathrm{Gm}(\mathbf{1 0})$ & $>64$ & $>64$ & $>64$ & $>64$ & $>64$ & $>64$ & $>64$ & $>64$ \\
\hline $\mathrm{K}-41 \mathrm{H}(\mathbf{1 1})$ & 16 & 16 & 16 & 8 & 8 & 32 & 16 & 16 \\
\hline Van & 0.5 & 1 & 1 & 1 & 1 & $>64$ & 0.25 & 1 \\
\hline Ery & 0.0625 & $>64$ & $>64$ & $>64$ & 0.0625 & 1 & 0.0625 & $>64$ \\
\hline Kan & 2 & 0.25 & $>64$ & 1 & 1 & 32 & 0.25 & $>64$ \\
\hline
\end{tabular}

${ }^{a}$ Notes: ML, Micrococcus luteus; Ljh13, Staphylococcus simulans AKA1; SA1, Staphylococcus aureus 16162; SA2, Staphylococcus aureus 16339; SA3, Staphylococcus aureus ATCC 29213; SA4, Staphylococcus aureus (cfr) GDQ6P012P; BS, Bacillus subtilis; MRSA, methicillin-resistant Staphylococcus aureus. Van, Vancomycin; Ery, Erythromycin; Kan, Kanamycin; Van, Ery and Kan served as positive controls. The tests were all performed in triplicate. 
Table S8. Strains or plasmids used and constructed in this study.

\begin{tabular}{|c|c|c|}
\hline Strains or plasmids & Description & Reference or source \\
\hline \multicolumn{3}{|l|}{ Strains } \\
\hline E. coli LE392 & $\begin{array}{l}\text { Host strain of cosmid vector } \\
\text { SuperCosI }\end{array}$ & Stratagene \\
\hline E. coli BW25113/pIJ790 & $\begin{array}{l}\text { Strain for homologous reorganization } \\
\text { during PCR-targeting process }\end{array}$ & 1 \\
\hline E. coli DH5 $\alpha / \mathrm{pIJ} 773$ & Host strain of plasmid pIJ773 & Stratagene \\
\hline E. coli & Strain for conjugation with & 2 \\
\hline ET12567/pUZ8002 & Streptomyces & 2 \\
\hline Streptomyces sp. SCSIO & Polyether antibiotic & Thic ctudy \\
\hline 01680 & K-41A-producing strain & inis stuay \\
\hline Streptomyces sp. SCSIO & pak4 gene mutant strain derived from & This study \\
\hline 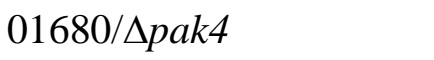 & Streptomyces sp. SCSIO 01680 & $1111 \mathrm{~s}$ study \\
\hline $\begin{array}{l}\text { Streptomyces sp. SCSIO } \\
01680 / \Delta \text { pak13 }\end{array}$ & $\begin{array}{l}\text { pak13 gene mutant strain derived } \\
\text { from Streptomyces sp. SCSIO } 01680\end{array}$ & This study \\
\hline $\begin{array}{l}\text { Streptomyces sp. SCSIO } \\
01680 / \Delta \text { pak15 }\end{array}$ & $\begin{array}{l}\text { pak15 gene mutant strain derived } \\
\text { from Streptomyces sp. SCSIO } 01680\end{array}$ & This study \\
\hline $\begin{array}{l}\text { Streptomyces sp. SCSIO } \\
01680 / \Delta \text { pak } 20\end{array}$ & $\begin{array}{l}\text { pak20 gene mutant strain derived } \\
\text { from Streptomyces sp. SCSIO } 01680\end{array}$ & This study \\
\hline $\begin{array}{l}\text { Streptomyces sp. SCSIO } \\
01680 / \Delta \text { pak31 }\end{array}$ & $\begin{array}{l}\text { pak31 gene mutant strain derived } \\
\text { from Streptomyces sp. SCSIO } 01680\end{array}$ & This study \\
\hline $\begin{array}{l}\text { Streptomyces sp. SCSIO } \\
01680 / \Delta \text { pak38 }\end{array}$ & $\begin{array}{l}\text { pak38 gene mutant strain derived } \\
\text { from Streptomyces sp. SCSIO } 01680\end{array}$ & This study \\
\hline \multicolumn{3}{|l|}{ Plasmids } \\
\hline SuperCosI & $A m p^{r}, \operatorname{Kan}^{r}$, cosmid vector & Stratagene \\
\hline pIJ790 & $\begin{array}{l}\mathrm{Cml}^{\mathrm{r}} \text {; gam, bet, exo encoding } \lambda \text {-RED } \\
\text { reorganization system for } \\
\text { PCR-targeting }\end{array}$ & 3 \\
\hline pIJ773 & $\begin{array}{l}\text { Apr }{ }^{\mathrm{r}} \text {, provider of } \operatorname{oriT/acc}(3) I V \\
\text { fragment }\end{array}$ & 3 \\
\hline pUZ8002 & $\begin{array}{l}\mathrm{Kan}^{\mathrm{r}} \text {, non-transmissible plasmid with } \\
\text { tra gene for conjugation }\end{array}$ & 4 \\
\hline
\end{tabular}


Table S9. Primers used in this study.

\begin{tabular}{|c|c|}
\hline Primers & Sequence (5' - 3') \\
\hline \multicolumn{2}{|c|}{ For genomic library screening } \\
\hline pak2-SF & AGCTTCTACTTCCCGGTCTCC \\
\hline pak2-SR & CCGGCCCAGGCACGGGCCTC \\
\hline pak12-SF & GCCACCGTCCCGGAGCGGCC \\
\hline pak12-SR & GGCCGCTCCGGGACGGTGGC \\
\hline pak27-SF & ACATCCTGTGGTCCGGGGGC \\
\hline pak27-SR & AGCCGGCCCCTCTGGCTCTC \\
\hline pak38-SF & CGGAAGAGGCCACCGACCGG \\
\hline pak38-SR & ACTCCCGGCGGCAGCGCAGG \\
\hline \multicolumn{2}{|c|}{ For construction of gene mutant strains } \\
\hline pak4-delF & $\begin{array}{l}\text { GGCACGGGCACGACTTTGGGTGACCCGATCGAGGCGCAGattc } \\
\text { cggggatccgtcgacc }\end{array}$ \\
\hline pak4-delR & $\begin{array}{l}\text { ACGACTGCGCAACGCCACAACCCGCGCACCATCCTCCAAtgta } \\
\text { ggctggagctgcttc }\end{array}$ \\
\hline pak4-testF & TCATCGGGTGCTCGCGGTCG \\
\hline pak4-testR & GGGCCATTGACGGCCGCGAT \\
\hline pak13-delF & $\begin{array}{l}\text { CGCGACGACGGCTTCTACCGCGACATCCTCGGCAACGACattcc } \\
\text { ggggatccgtcgacc }\end{array}$ \\
\hline pak13-delF & $\begin{array}{l}\text { GGCGCACCGCTCCCGCACGTACTCCGCGTTCGGCACCAGtgta } \\
\text { ggctggagctgcttc }\end{array}$ \\
\hline pak13-testF & CCTCCCAGGGCAAGCTGGCG \\
\hline pak13-testR & TCTTCTGCTTGGAGGAGCGG \\
\hline pak15-delF & $\begin{array}{l}\text { ACCAACCGCTTCACCGACATGCTGGCCGCGCGGCTCAGGattc } \\
\text { cggggatccgtcgacc }\end{array}$ \\
\hline pak15-delF & $\begin{array}{l}\text { ACCGTCGAGCAGCAGGTTGAAGGTCCGCTTGGTGTTCTCtgta } \\
\text { ggctggagctgcttc }\end{array}$ \\
\hline pak15-testF & GCACTTCGGGTACTGGGACG \\
\hline pak15-testR & TCCTCGGCCGTGATGCCGTG \\
\hline pak20-delF & $\begin{array}{l}\text { GCACGACTGCGGGTCGGCCCGGACTCCCGTGTCCTCGACattc } \\
\text { cggggatccgtcgacc }\end{array}$ \\
\hline pak20-delF & $\begin{array}{l}\text { CAACTCGTCCAGCCGCAGCCCGGCCGCGGAGACCAGGTCtgta } \\
\text { ggctggagctgcttc }\end{array}$ \\
\hline pak20-testF & CCACCTCGGGTACTGGGAAG \\
\hline pak20-testR & GCGCCGGTCGATGAAGCCGT \\
\hline pak31-delF & $\begin{array}{l}\text { CTCGCACTCTGGGACCCGACGACGAACGGAGTGCGGTATattcc } \\
\text { ggggatccgtcgacc }\end{array}$ \\
\hline pak31-delF & $\begin{array}{l}\text { CGGAATACCGCGGCCCTCCCTGACGCAGGTCCACGCGTCtgtag } \\
\text { gctggagctgcttc }\end{array}$ \\
\hline pak31-testF & CGACGAACGGAGTGCGGTAT \\
\hline pak31-testR & ATAGCTCCACGGAATACCGC \\
\hline pak38-delF & $\begin{array}{l}\text { CCGGAAGAGGCCACCGACCGGTTCACCGACCTGCTCGCGattc } \\
\text { cggggatcegtcgacc }\end{array}$ \\
\hline pak38-delF & $\begin{array}{l}\text { CCGGCGGCAGCGCAGGATCCCGTCGATCACCCGCATGAAtgta } \\
\text { ggctggagctgcttc }\end{array}$ \\
\hline pak38-testF & ACCGACTACTACAGCGCGCT \\
\hline pak38-testR & TCGACGCTGACGCCGTGTTC \\
\hline
\end{tabular}



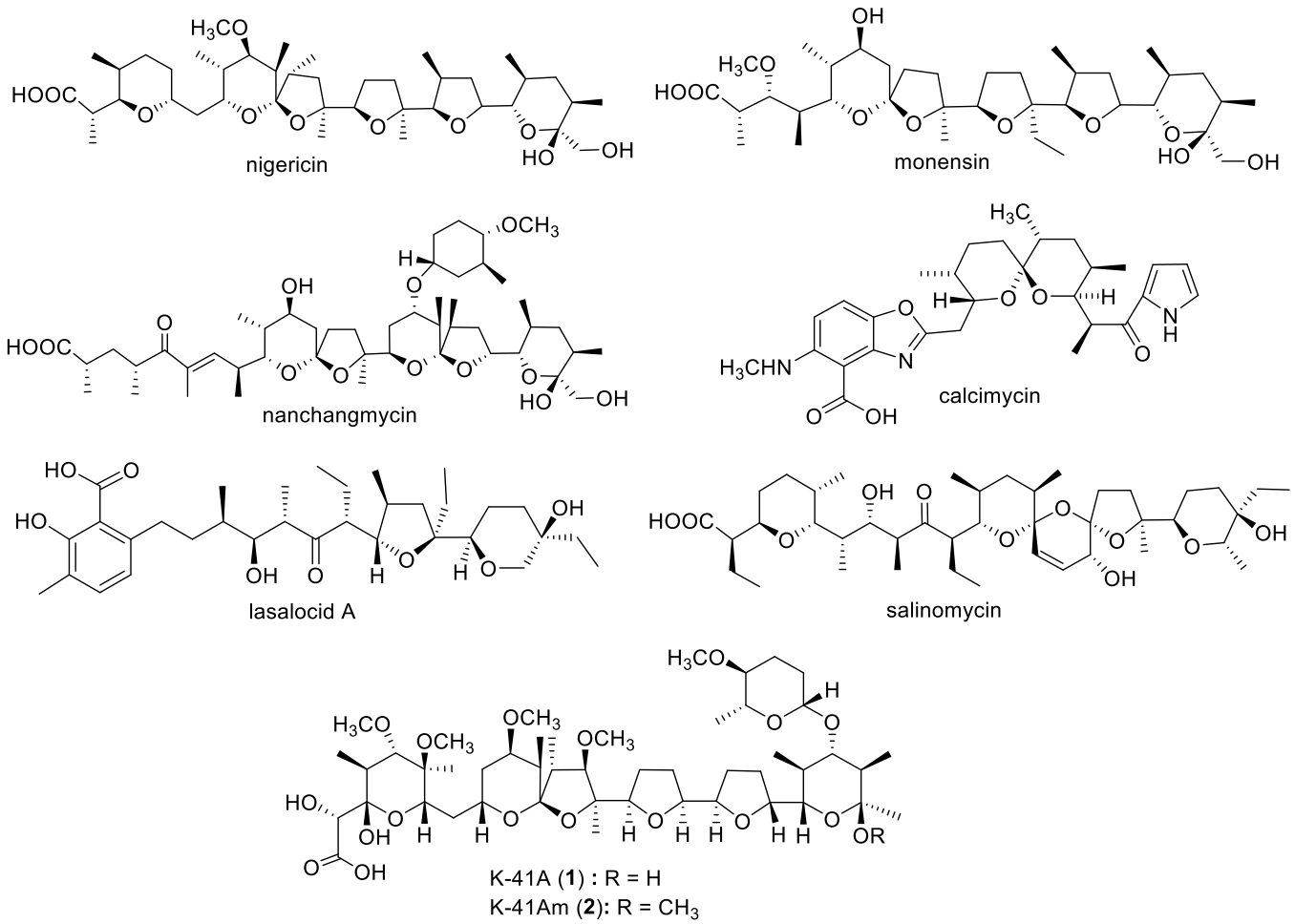

Figure S1. Previously reported polyether compounds as well as the polyether antibiotics K-41A and K-41Am isolated from Streptomyces sp. SCSIO 01680.

A

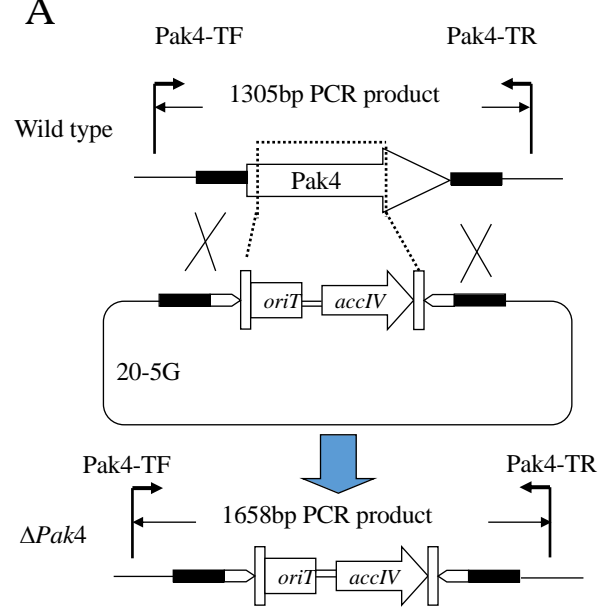

B

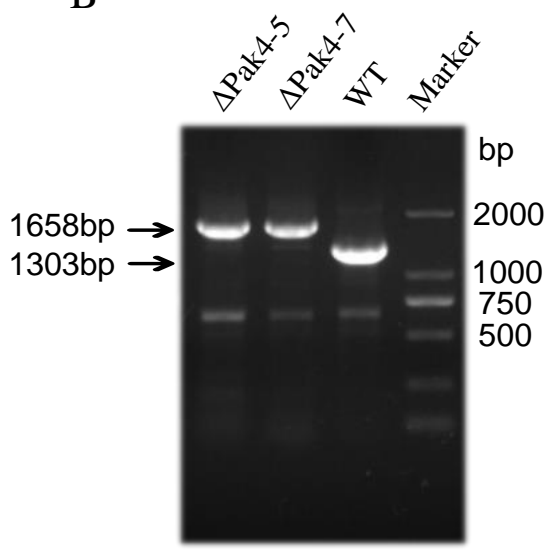

Figure S2. pak4 gene disruption in Streptomyces sp. SCSIO 01680 via PCR-targeting technique. (A) Schematic diagram of pak4 gene disruption. (B) Genomic comparison of the WT strain and the pak4 gene double-cross mutant strain by PCR using the primers listed in Table S2. WT: PCR result when the genomic DNA from WT strain as PCR template; $\triangle$ Pak4: PCR result using the genomic DNA from pak4 gene mutant strain as PCR template. 


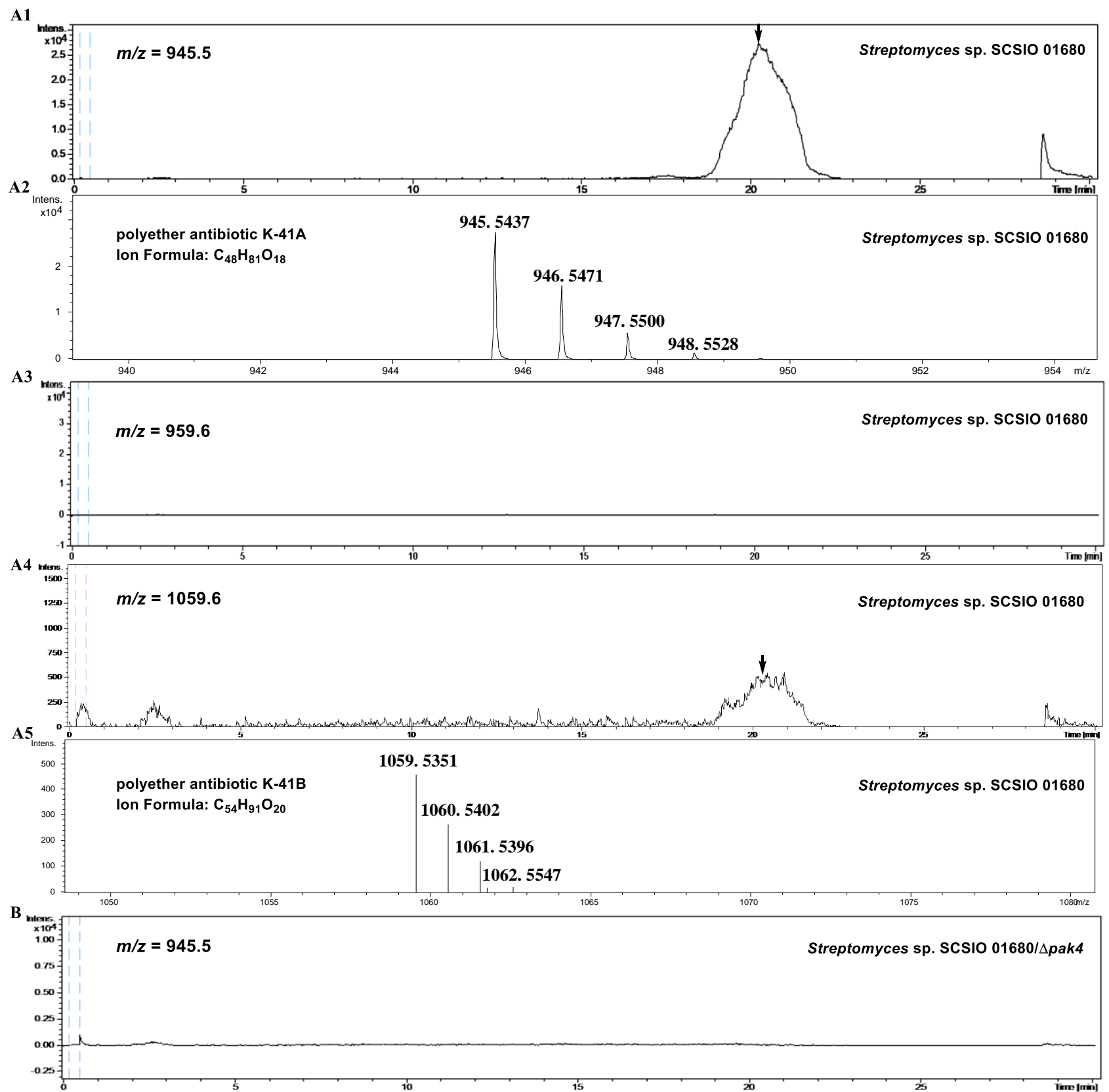

Figure S3. HR-ESI-LC-MS of fermentation extracts from WT Streptomyces sp. SCSIO 01680 and ketosynthase gene (pak4) disrupted mutant strain Streptomyces sp. SCSIO 01680/Apak4. A1. LC-MS of WT selected for $\mathrm{m} / z=945.5$ corresponding to K-41A; A2. The corresponding MS spectrum of the peak pointed by the arrow in figure A1; A3. LC-MS of WT selected for $m / z=959.6$ corresponding to K-41Am; A4. LC-MS of WT selected for $m / z=1059.6$ corresponding to K-41B; A5. The corresponding MS spectrum of the peak pointed by the arrow in figure A4; B. LC-MS of mutant strain selected for $m / z=945.5(\mathrm{~K}-41 \mathrm{~A})$. 

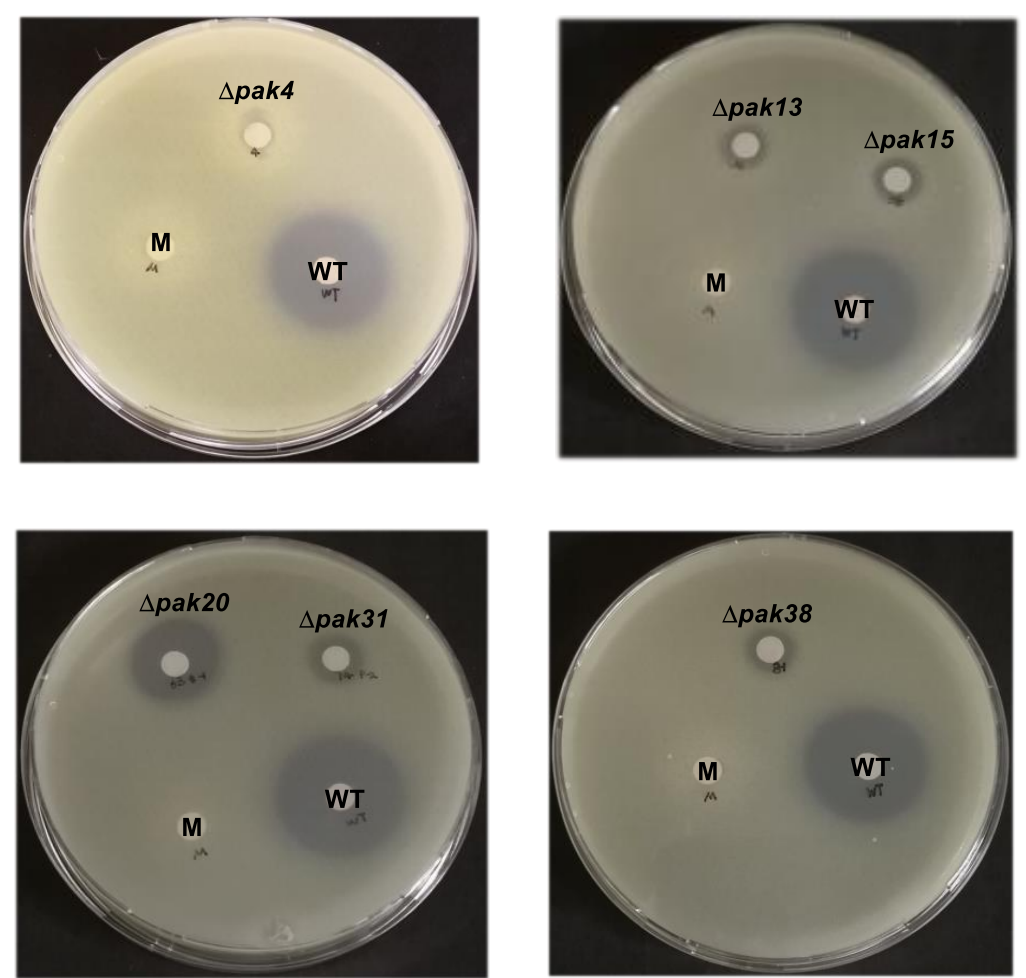

Figure S4. The biological activity test (disc diffusion) against Micrococcus luteus. WT: extract from Streptomyces sp. SCSIO 01680 (positive control); M: methanol (negative control); pak4: extract from $\Delta p a k 4$ mutant; $\Delta p a k 13$ : extract from $\Delta p a k 13$ mutant; $\Delta$ pak15: extract from $\Delta p a k 15$ mutant; $\Delta p a k 20$ : extract from $\Delta p a k 20$ mutant; $\Delta p a k 31$ : extract from $\Delta p a k 31$ mutant; $\Delta p a k 38$ : extract from $\Delta$ pak38 mutant. 

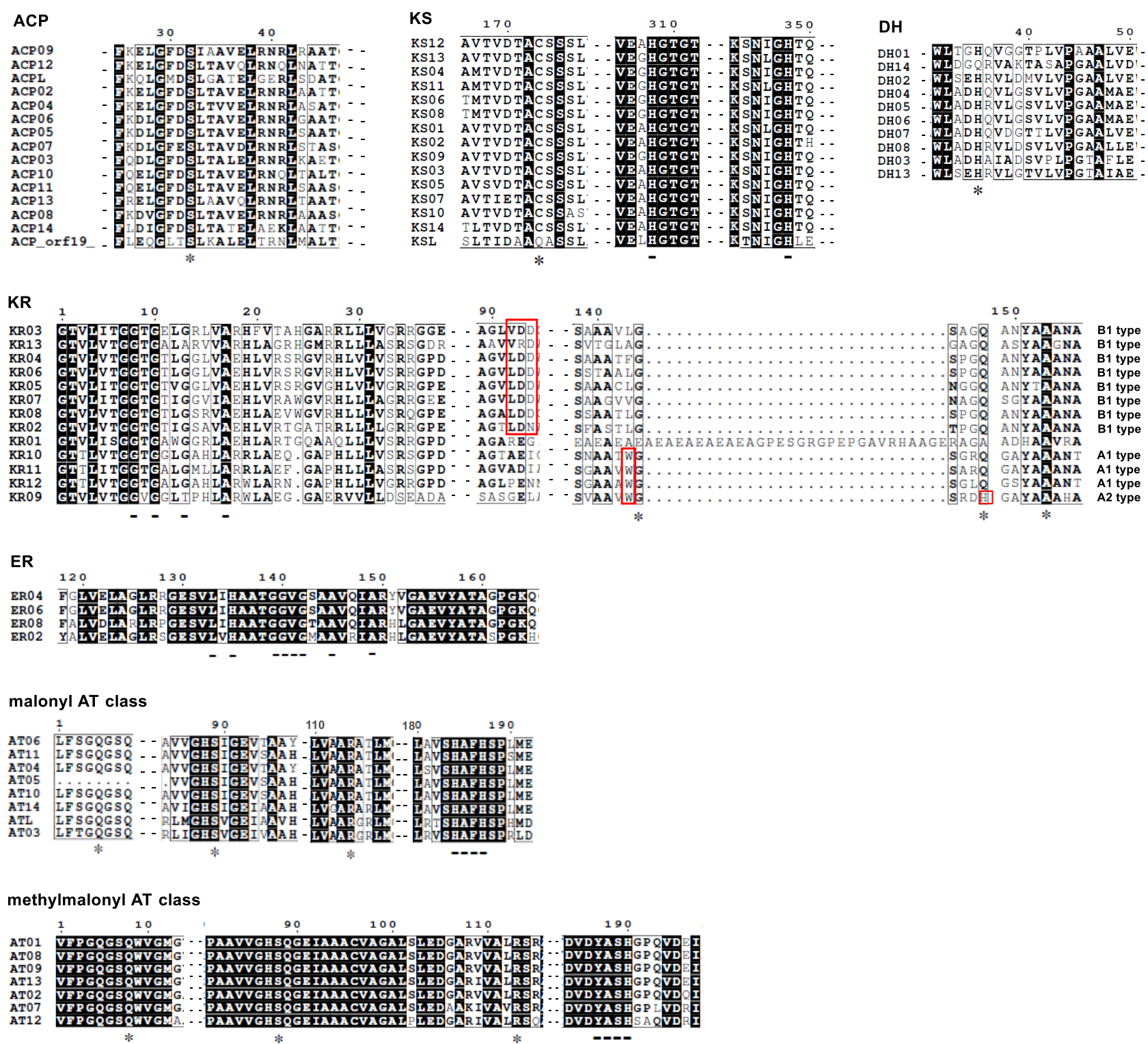

Figure S5. Alignment of sequences of PKS domains from polyether antibiotic K-41A gene cluster, but only the regions containing the proposed active sites are shown. Active site residues are marked with asterisks, conserved motifs vital to the function in each domain are underlined. Additionally, the characteristic residues of the A-type and B-type KR domains have been framed by solid lines. 

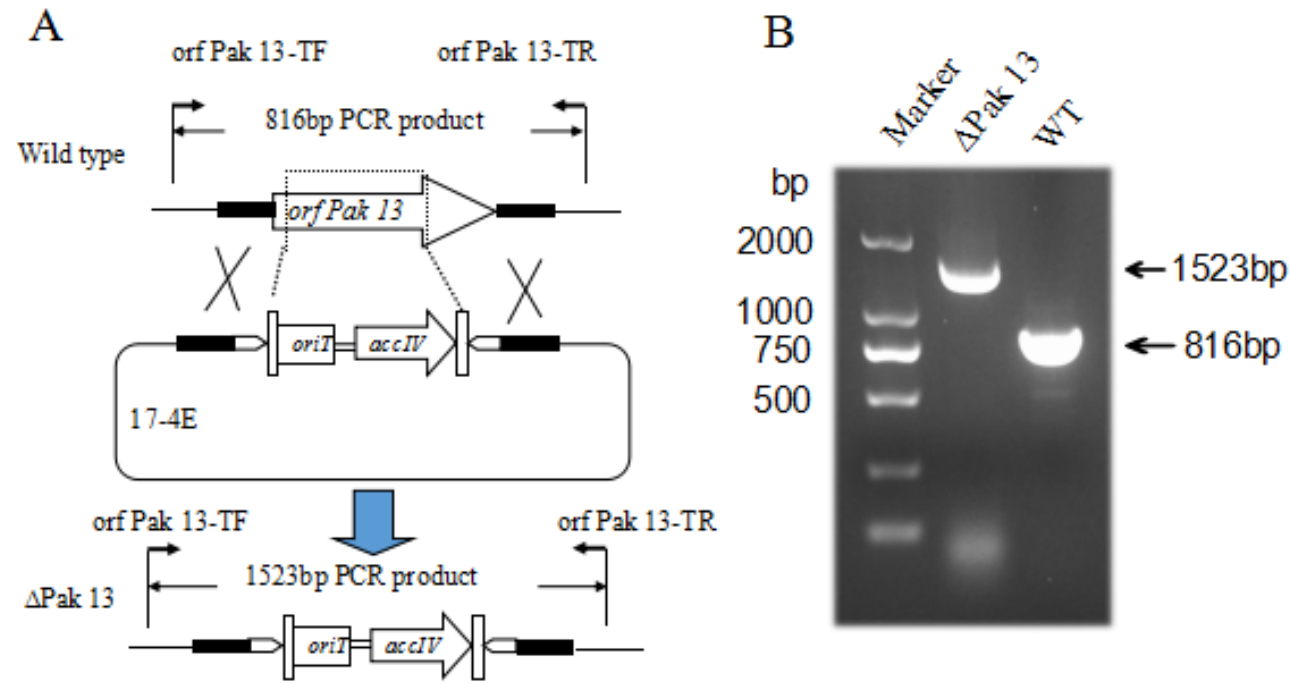

Figure S6. pak13 gene disruption in Streptomyces sp. SCSIO 01680 via PCR-targeting technique. (A) Schematic diagram of pak13 gene disruption. (B) Genomic comparison of the WT strain and the pak13 gene double-cross mutant strain by PCR using the primers listed in Table S2. WT: PCR result when the genomic DNA from WT strain as PCR template; $\triangle \mathrm{Pak} 13$ : PCR result using the genomic DNA from pak13 gene mutant strain as PCR template.
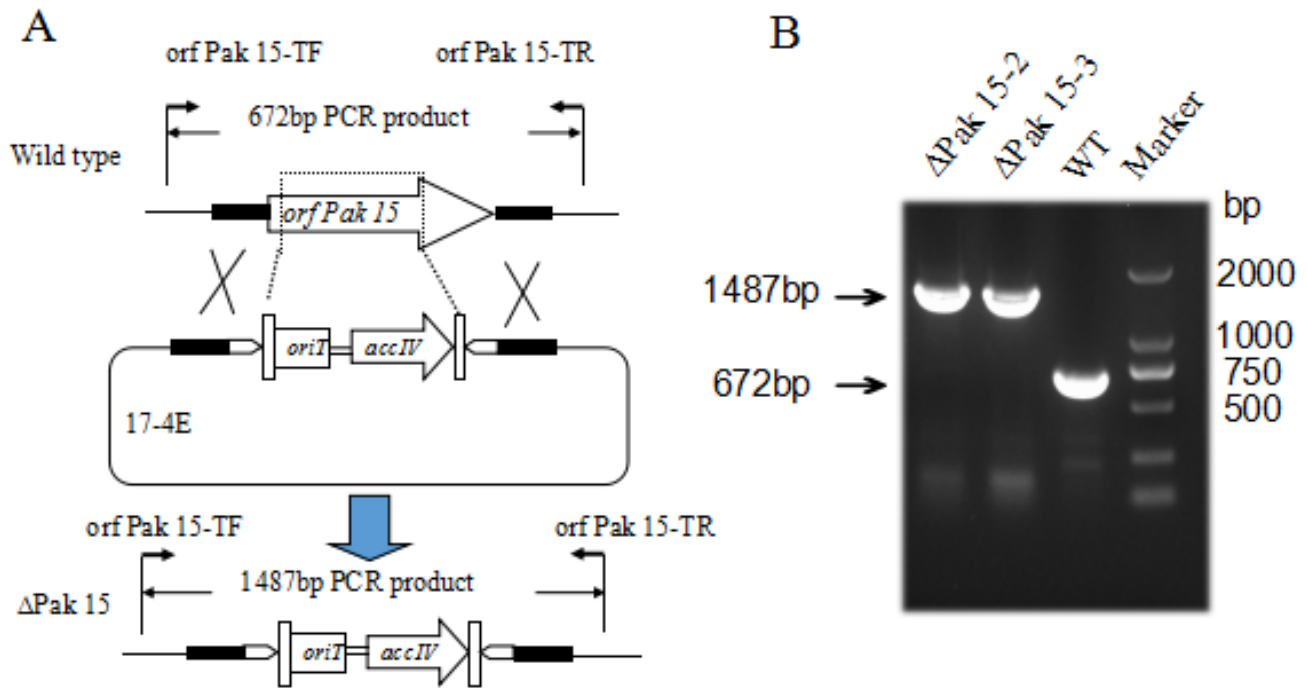

Figure S7. pak15 gene disruption in Streptomyces sp. SCSIO 01680 via PCR-targeting technique. (A) Schematic diagram of pak15 gene disruption. (B) Genomic comparison of the WT strain and the pak15 gene double-cross mutant strain by PCR using the primers listed in Table S2. WT: PCR result when the genomic DNA from WT strain as PCR template; $\triangle \mathrm{Pak} 15$ : PCR result using the genomic DNA from pak15 gene mutant strain as PCR template. 


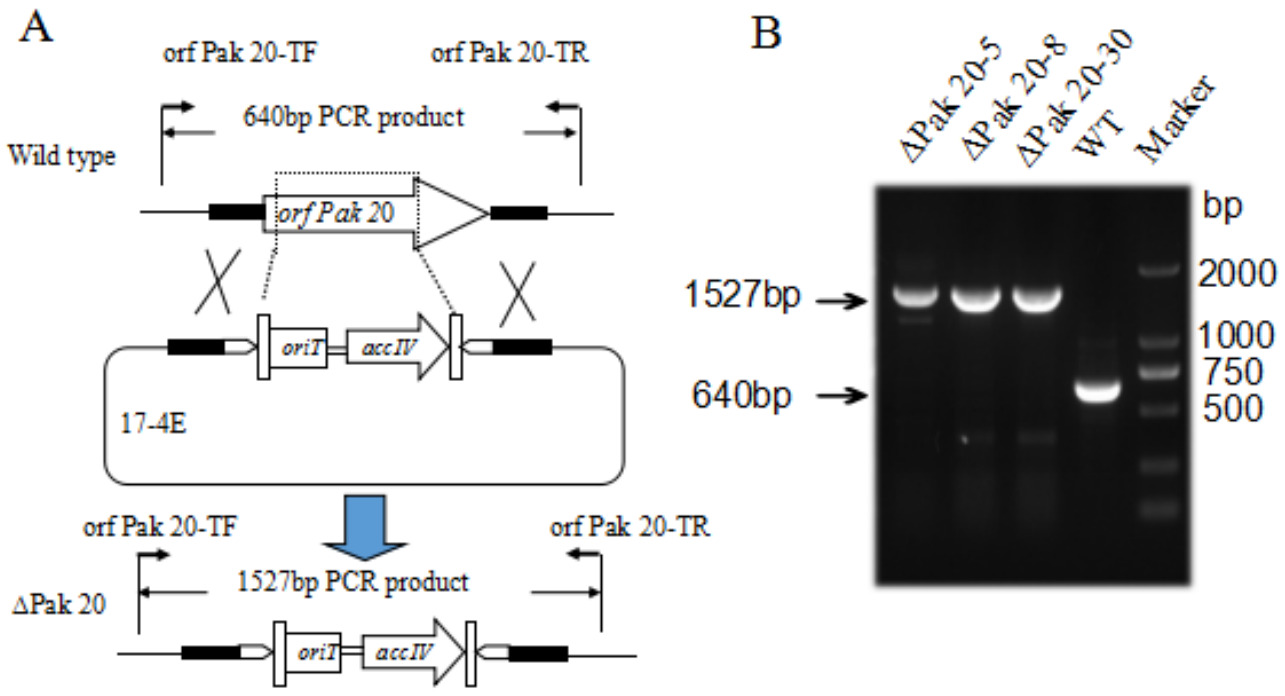

Figure S8. pak20 gene disruption in Streptomyces sp. SCSIO 01680 via PCR-targeting technique. (A) Schematic diagram of pak20 gene disruption. (B) Genomic comparison of the WT strain and the pak20 gene double-cross mutant strain by PCR using the primers listed in Table S2. WT: PCR result when the genomic DNA from WT strain as PCR template; $\triangle$ Pak20: PCR result using the genomic DNA from pak20 gene mutant strain as PCR template.

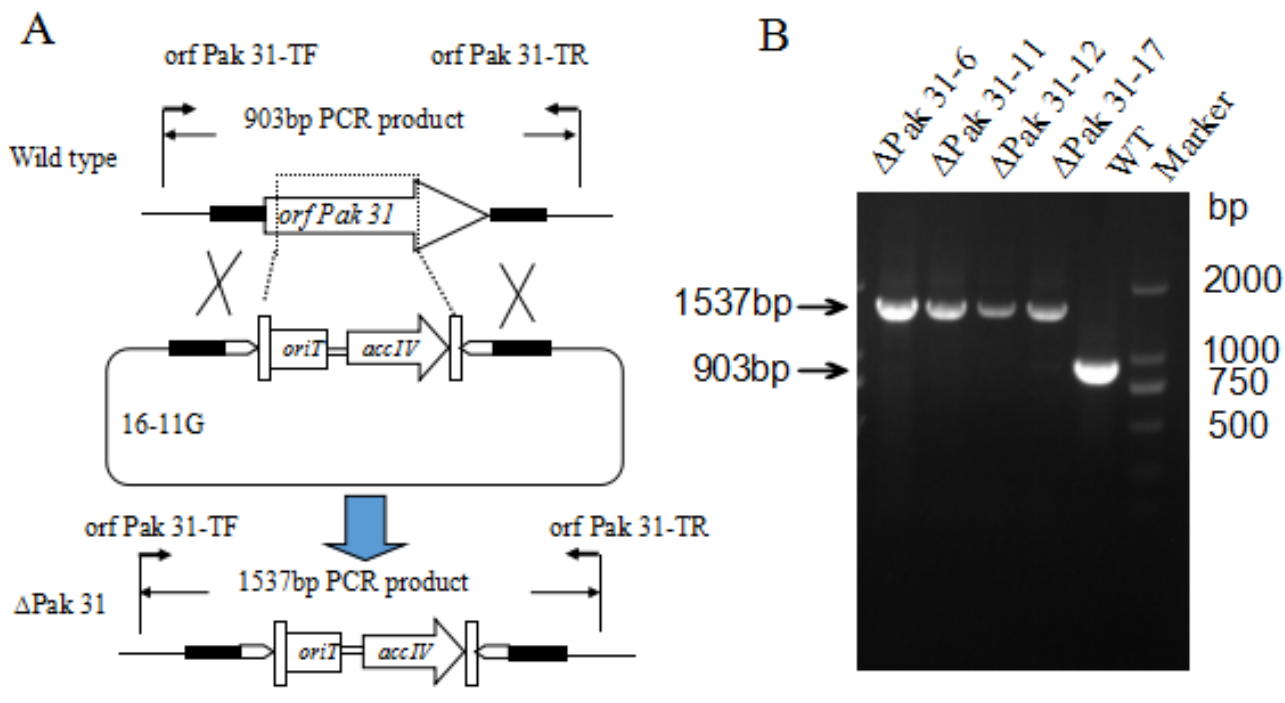

Figure S9. pak31 gene disruption in Streptomyces sp. SCSIO 01680 via PCR-targeting technique. (A) Schematic diagram of pak31 gene disruption. (B) Genomic comparison of the WT strain and the pak31 gene double-cross mutant strain by PCR using the primers listed in Table S2. WT: PCR result when the genomic DNA from WT strain as PCR template; $\triangle \mathrm{Pak} 31$ : PCR result using the genomic DNA from pak31 gene mutant strain as PCR template. 

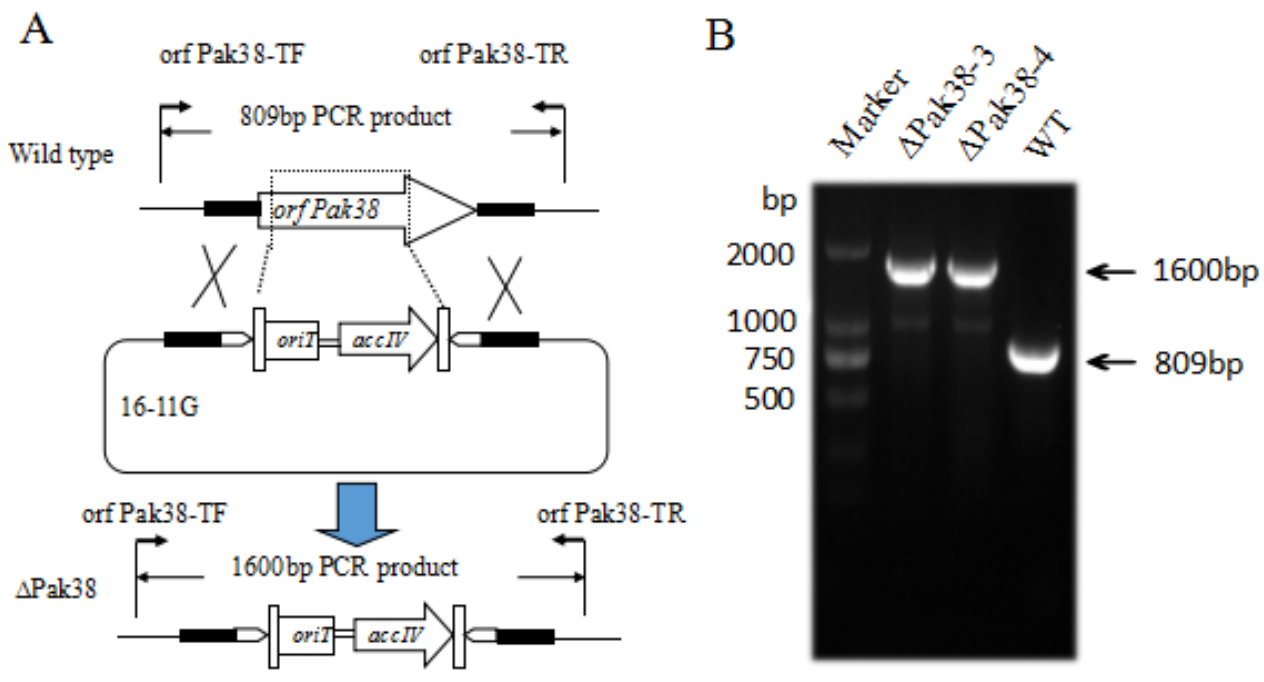

Figure S10. pak38 gene disruption in Streptomyces sp. SCSIO 01680 via PCR-targeting technique. (A) Schematic diagram of pak38 gene disruption. (B) Genomic comparison of the WT strain and the pak38 gene double-cross mutant strain by PCR using the primers listed in Table S2. WT: PCR result when the genomic DNA from WT strain as PCR template; $\triangle$ Pak38: PCR result using the genomic DNA from pak38 gene mutant strain as PCR template. 


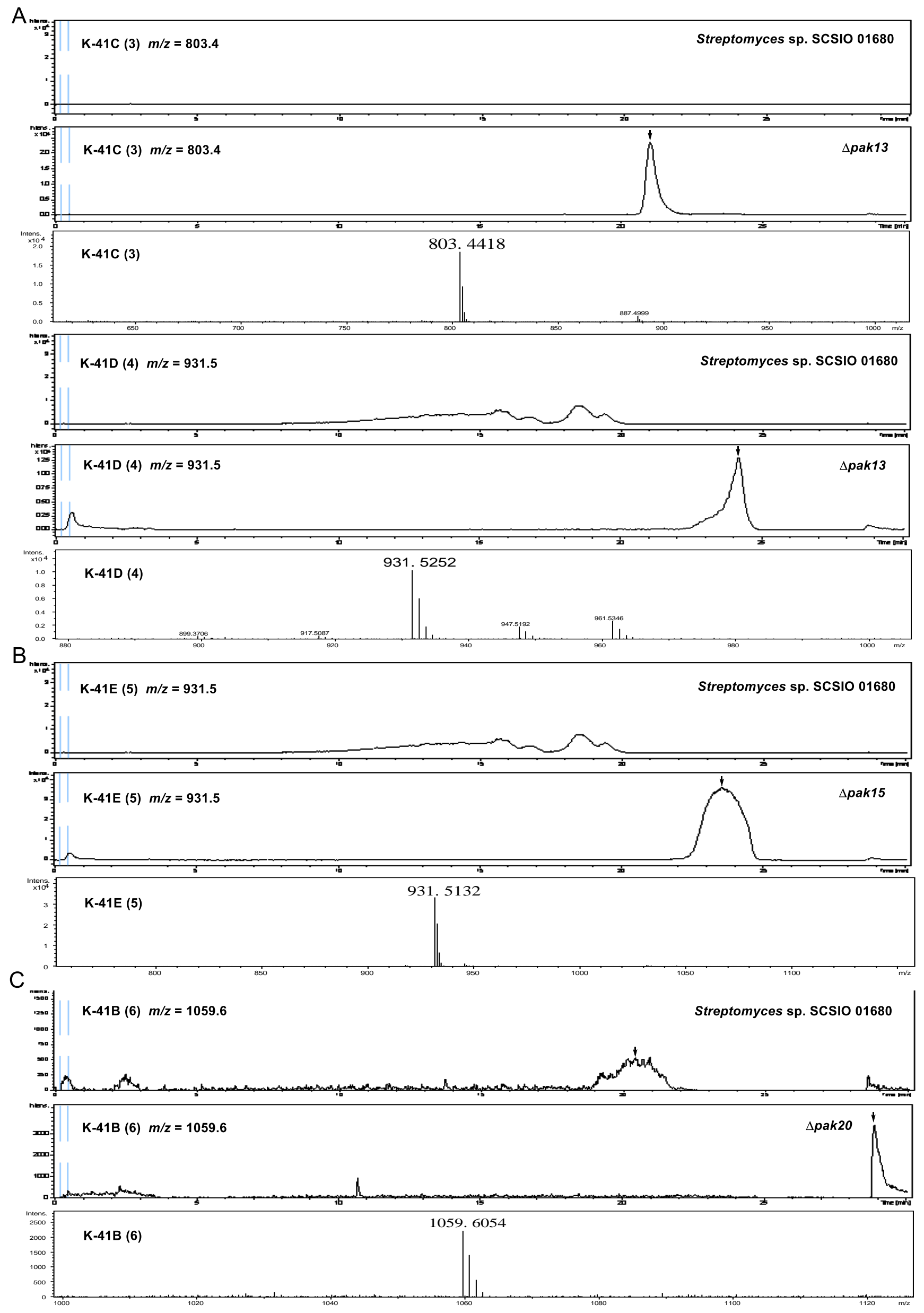



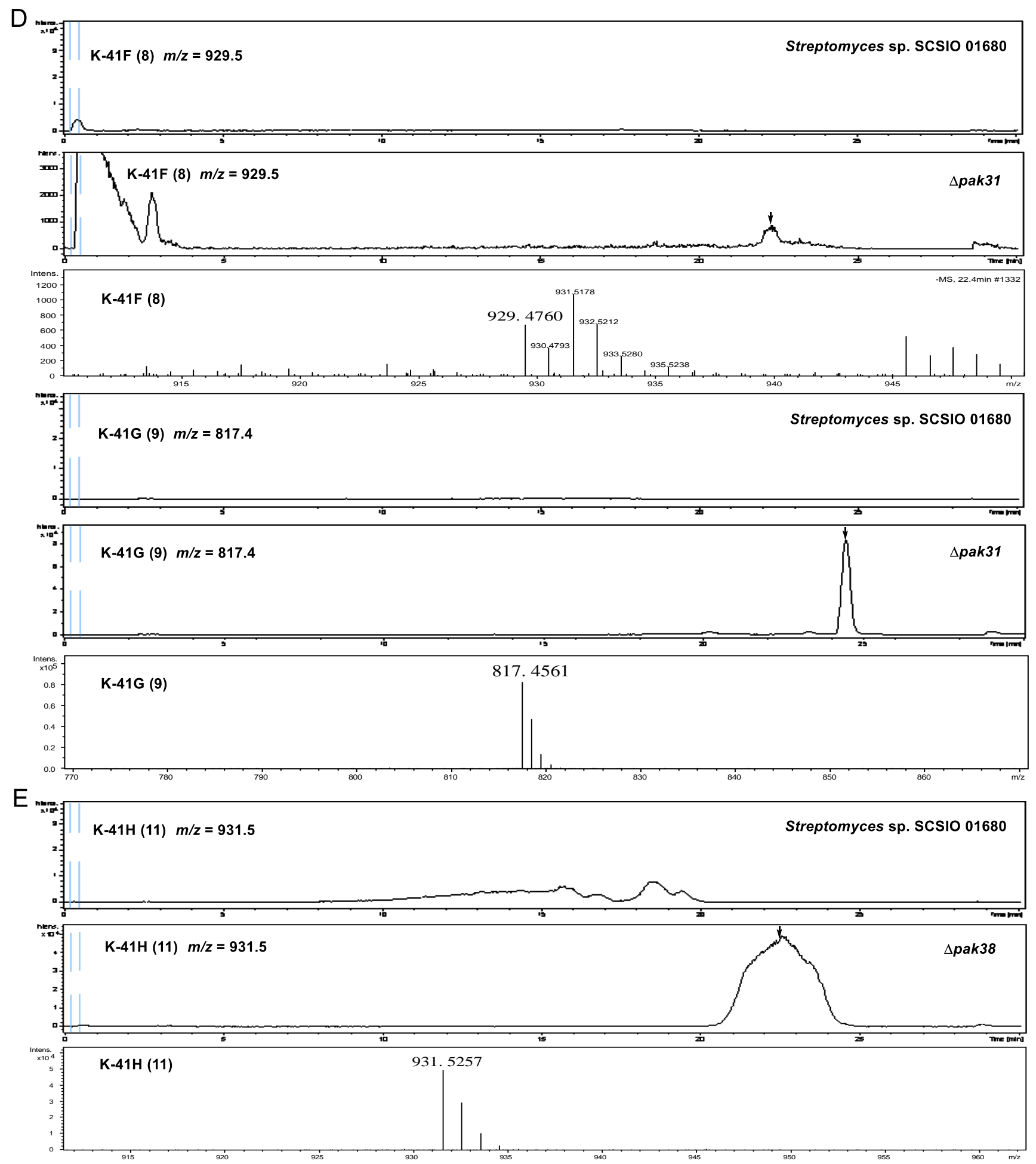

Figure S11. HR-ESI-LC-MS profiles of the fermentation extracts of five methyltransferase gene mutant strains: (A) $\Delta p a k 13$ mutant, (B) $\Delta p a k 15$ mutant, (C) $\Delta p a k 20$ mutant, (D) $\Delta p a k 31$ mutant, (E) $\Delta$ pak38 mutant. The ion peaks of $\mathrm{K}-41 \mathrm{~A}$ derivatives were pointed out by arrows and their corresponding MS spectra were also presented. 
A

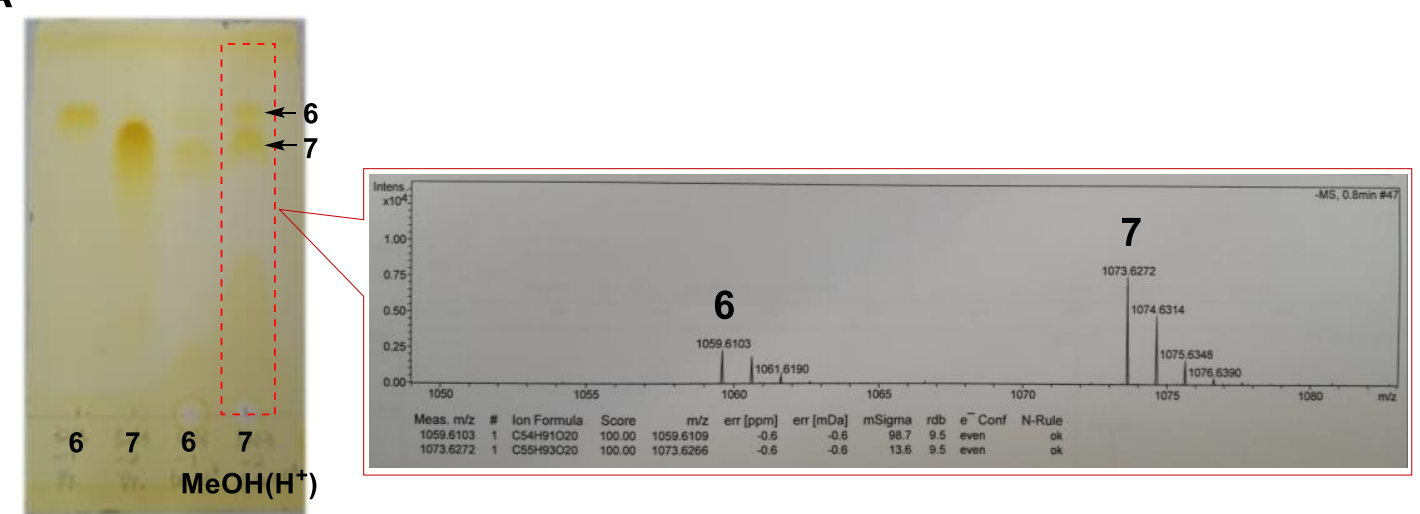

B

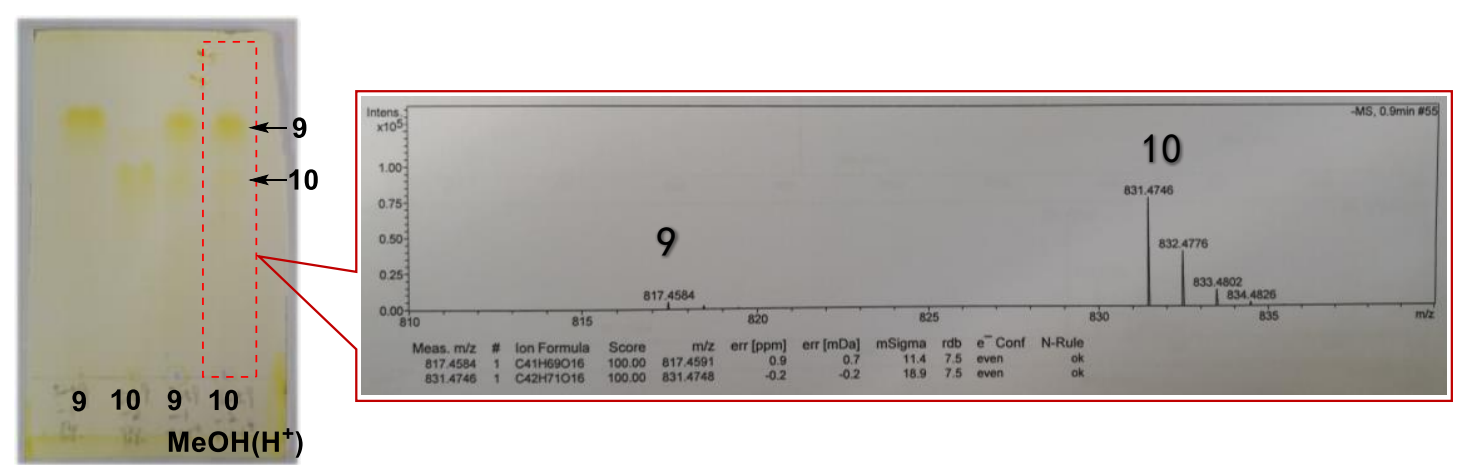

Figure S12. The TLC and HR-ESI-MS (NEG) analysis results of methanol immersion tests using compound 6, 7 (panel A) and 9,10 (panel B).
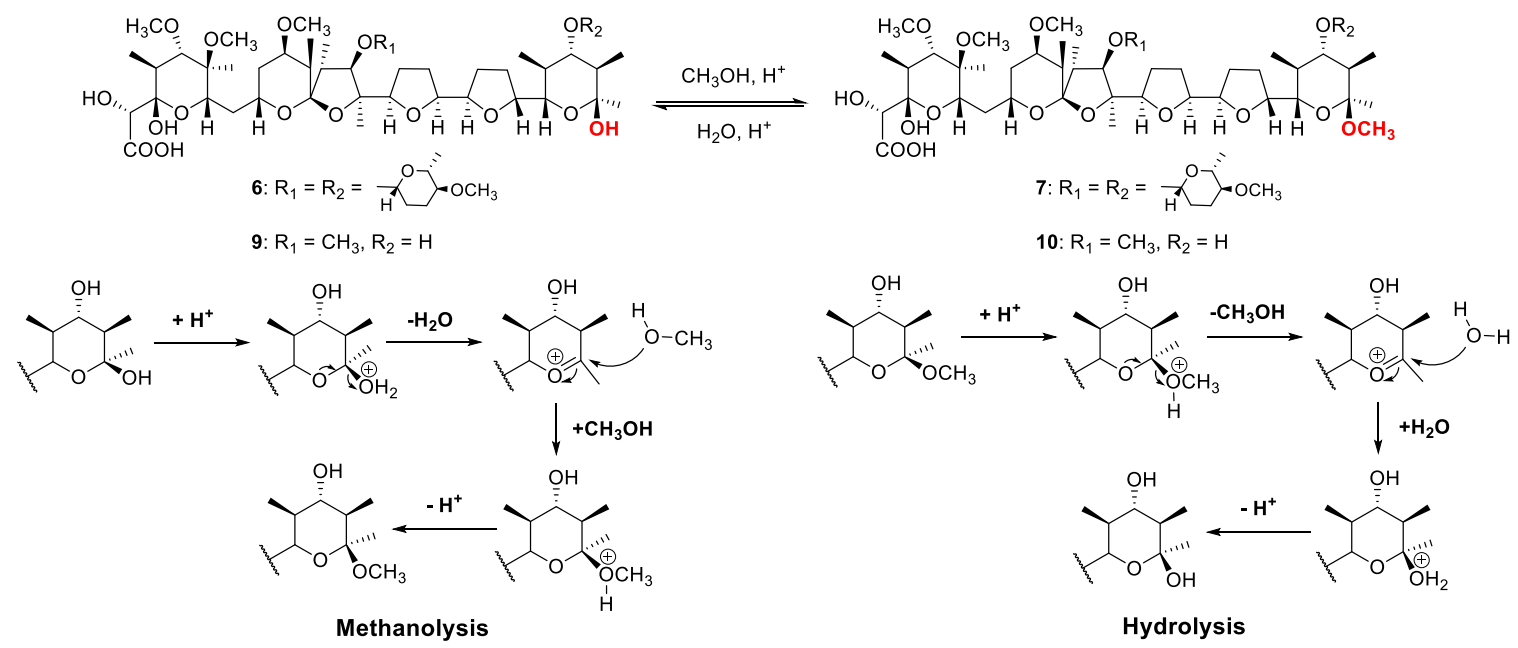

Figure S13. Plausible interconversion mechanisms (methanolysis and hydrolysis) of 6/7 and 9/10. 


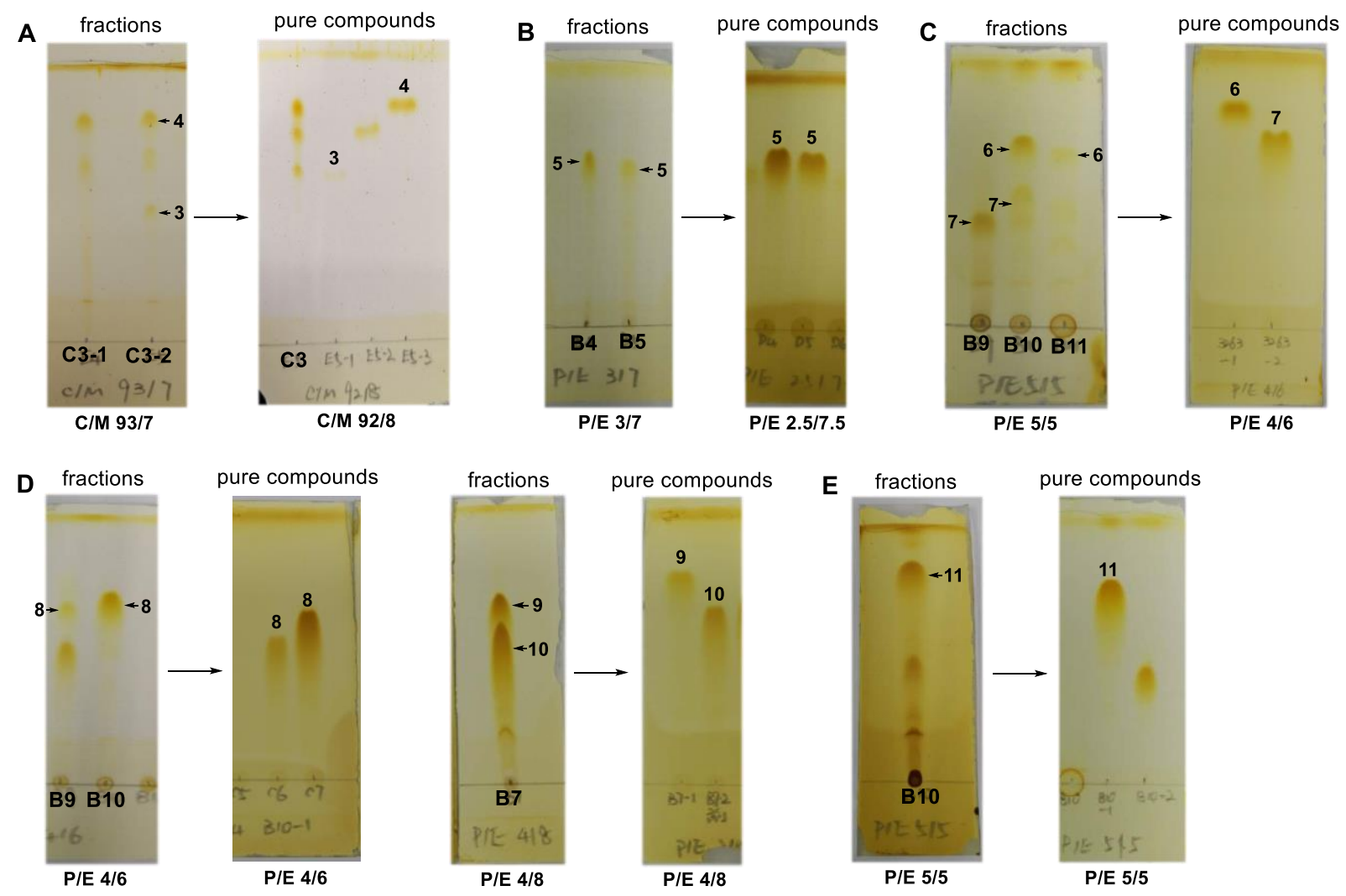

Figure S14. The TLC detection (iodine staining) of partially purified fractions and finally purified compounds 3-11 from extracts of five methyltransferase gene mutant strains $\operatorname{\Delta pak13}$ (A), $\Delta$ pak15 (B), $\Delta p a k 20$ (C), $\Delta p a k 31$ (D) and $\Delta p a k 38$ (E). C/M: Chloroform/Methanol; P/E: Petroleum ether/Ethyl acetate. 
Figure S15. HR-ESI-MS spectrum of compound 3 in $\mathrm{MeOH}$.

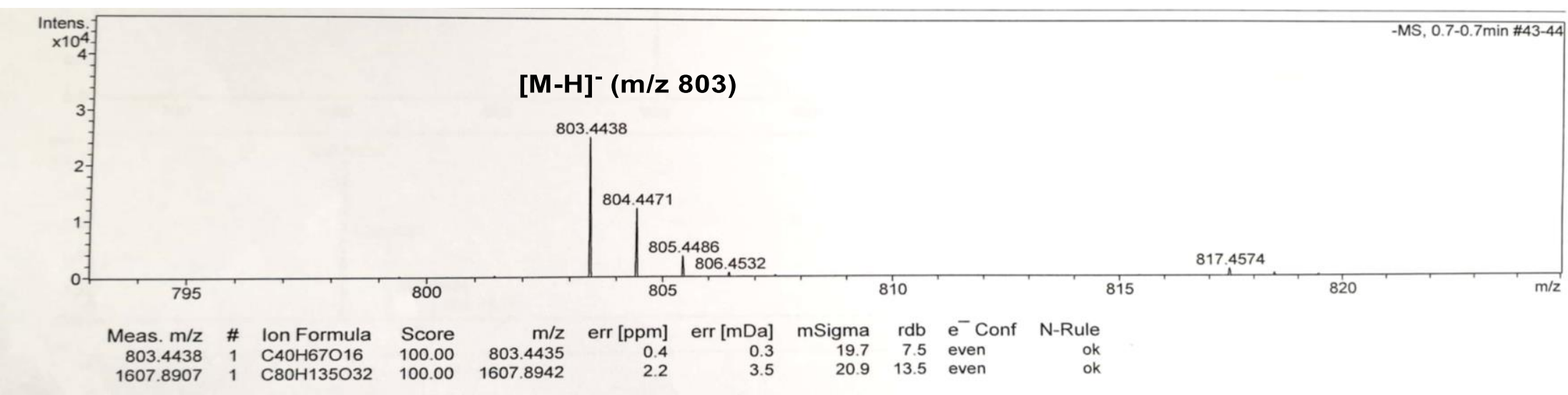

Figure S16. IR spectrum of compound 3 .

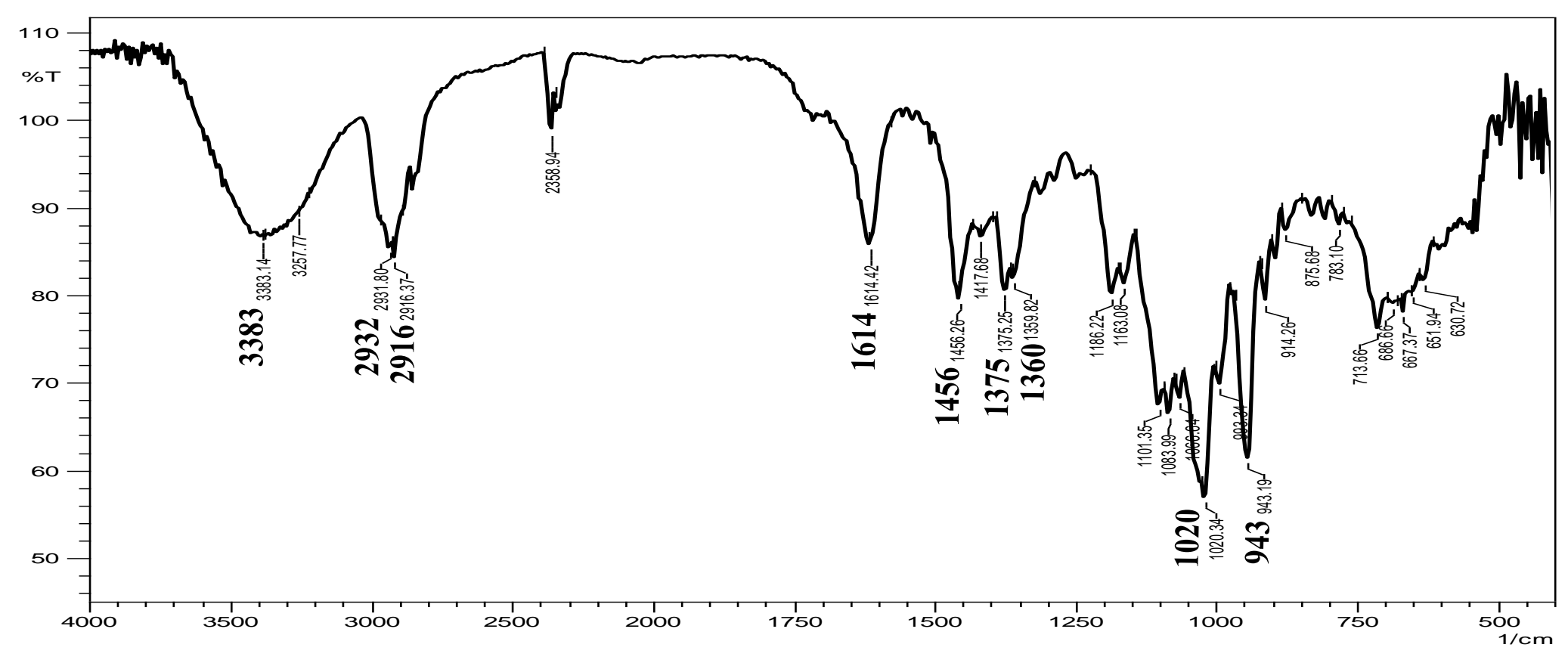


Figure S17. ${ }^{1} \mathrm{H}$ NMR $(500 \mathrm{MHz})$ spectrum of compound 3 in $\mathrm{CDCl}_{3}$.

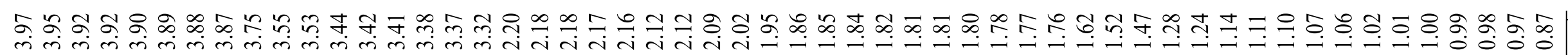

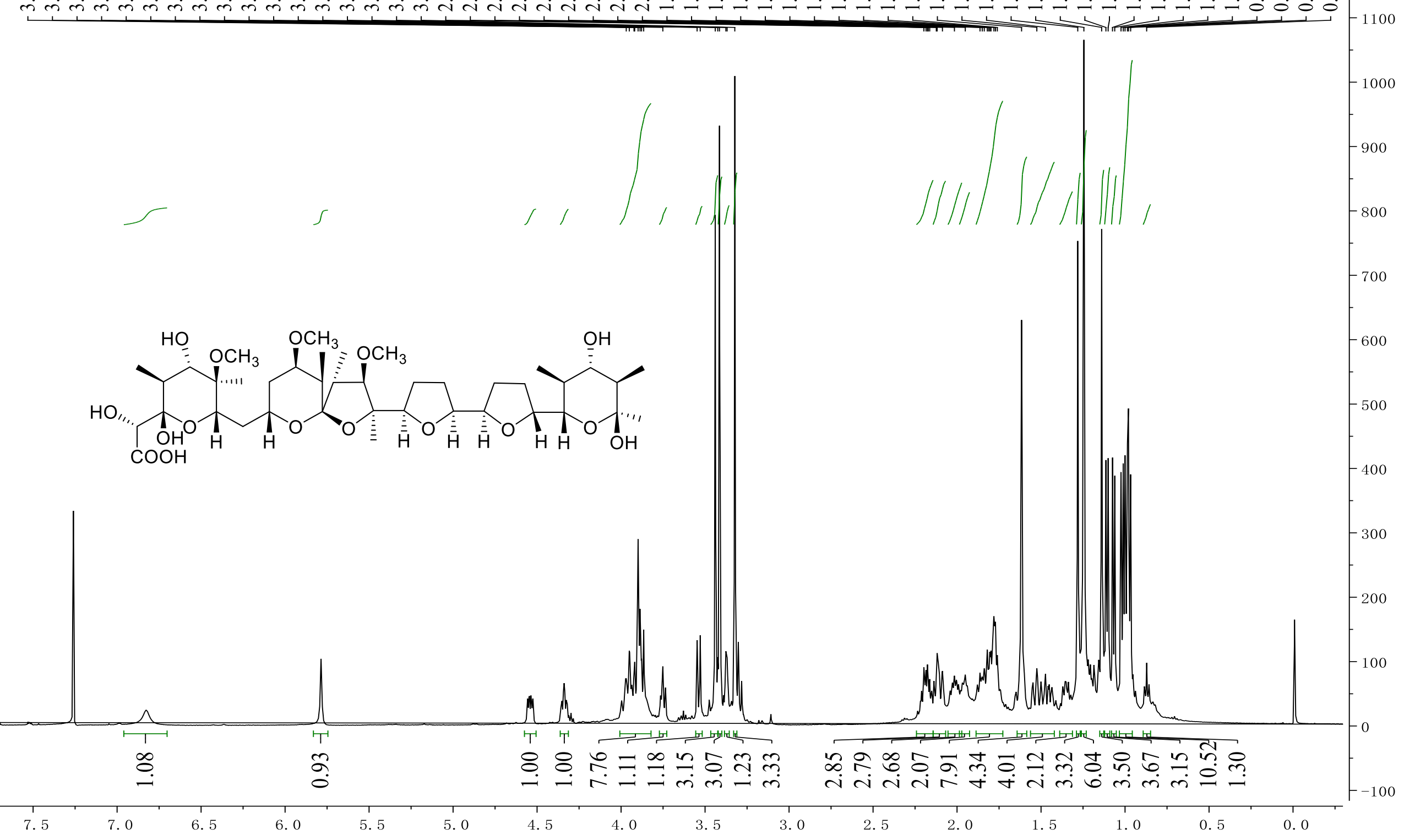


Figure S18. ${ }^{13} \mathrm{C}$ NMR $(125 \mathrm{MHz})$ spectrum of compound 3 in $\mathrm{CDCl}_{3}$.

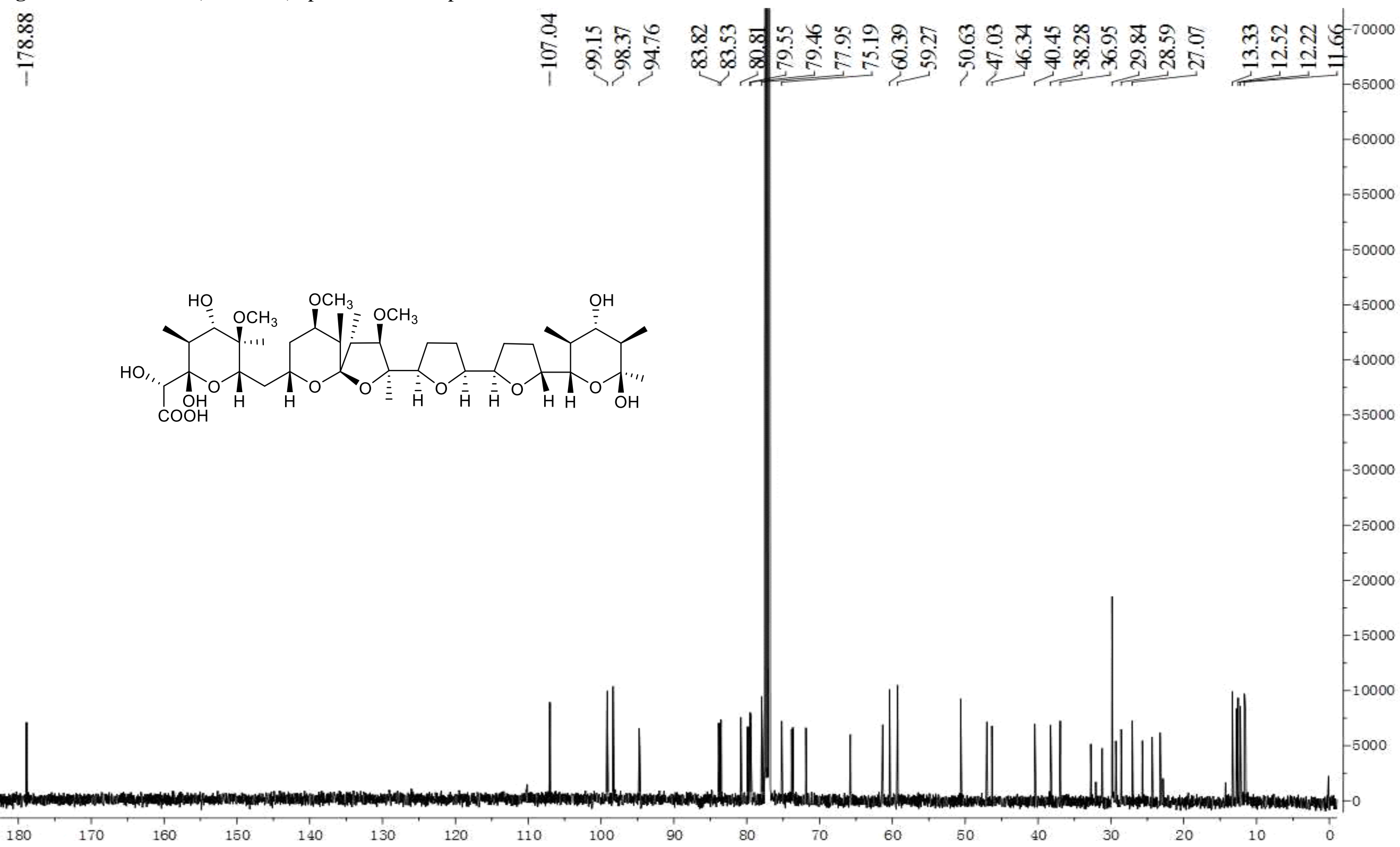


Figure S19. ${ }^{13} \mathrm{C}$ DEPT spectrum of compound 3 in $\mathrm{CDCl}_{3}$.
$\stackrel{\circ}{\stackrel{2}{\sigma}}$

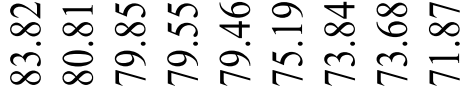
ำ กิก

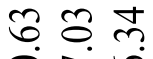
ๆ
跎守
유 ๗ூ
100 in
$1>1$
111 रूत

ㄷํ요 요 17000

드키 16000

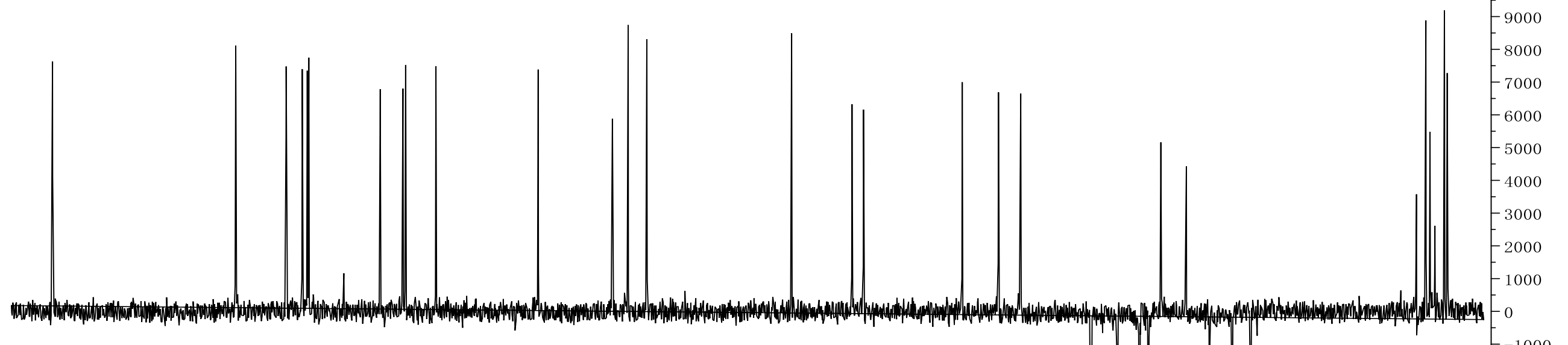

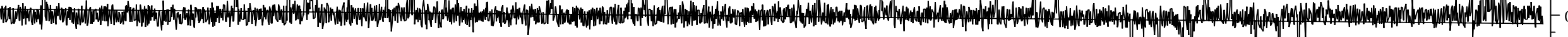
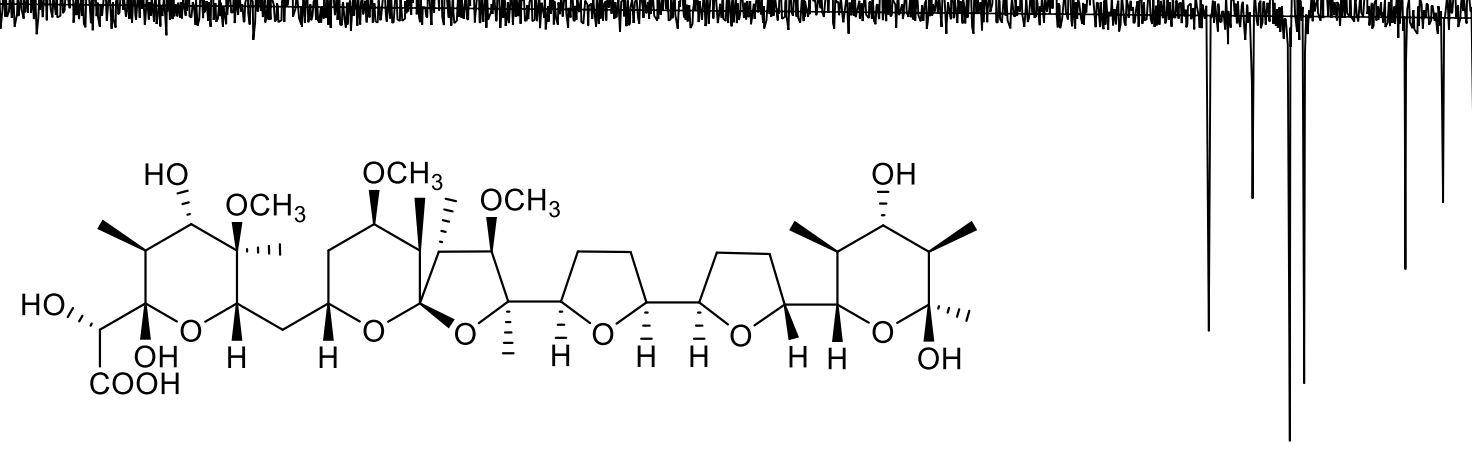
Figure S20. ${ }^{1} \mathrm{H}-{ }^{1} \mathrm{H}$ COSY spectrum of compound $\mathbf{3}$ in $\mathrm{CDCl}_{3}$.

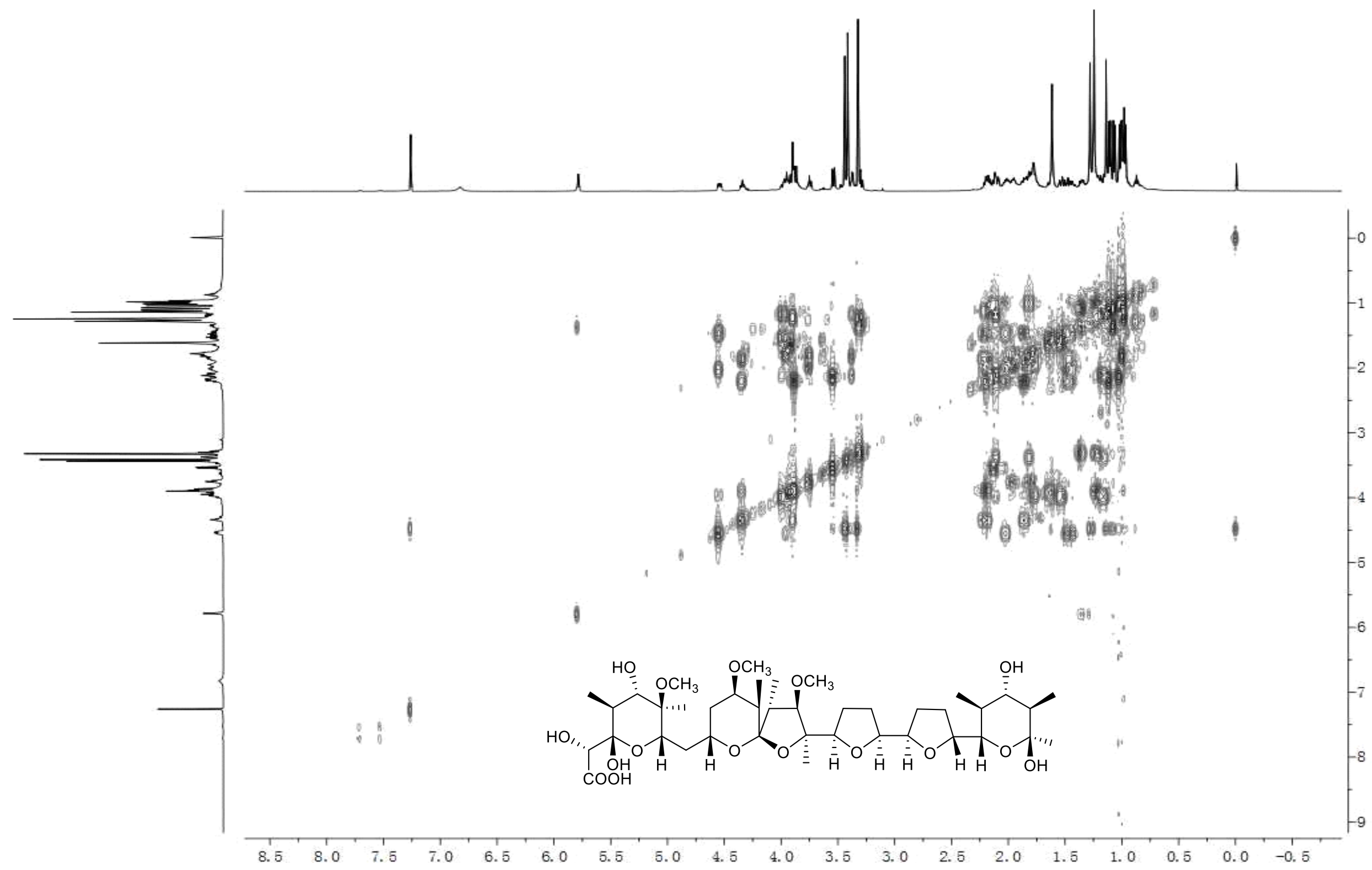


Figure S21. HSQC spectrum of compound 3 in $\mathrm{CDCl}_{3}$.

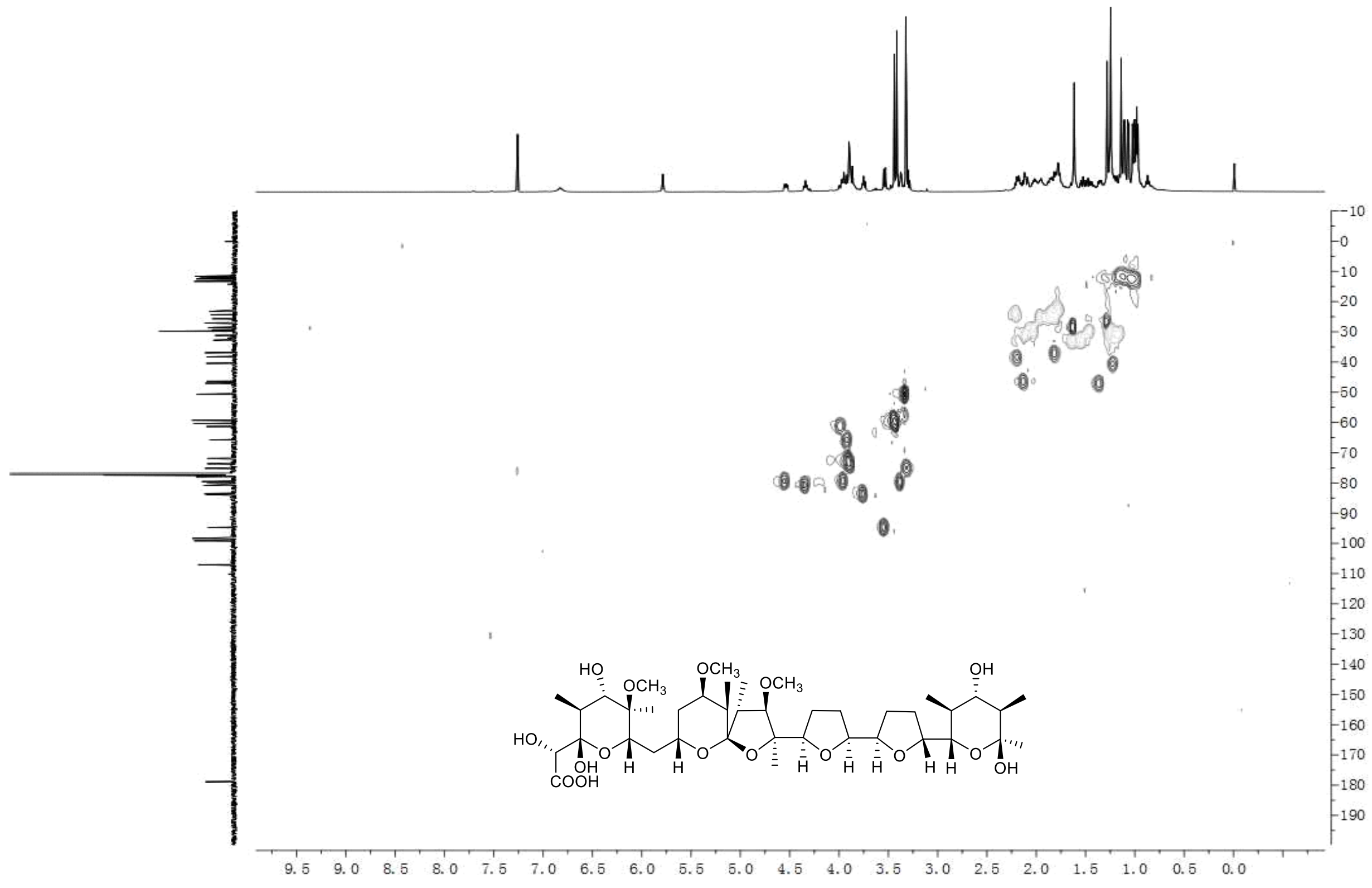


Figure S22. HMBC spectrum of compound $\mathbf{3}$ in $\mathrm{CDCl}_{3}$.

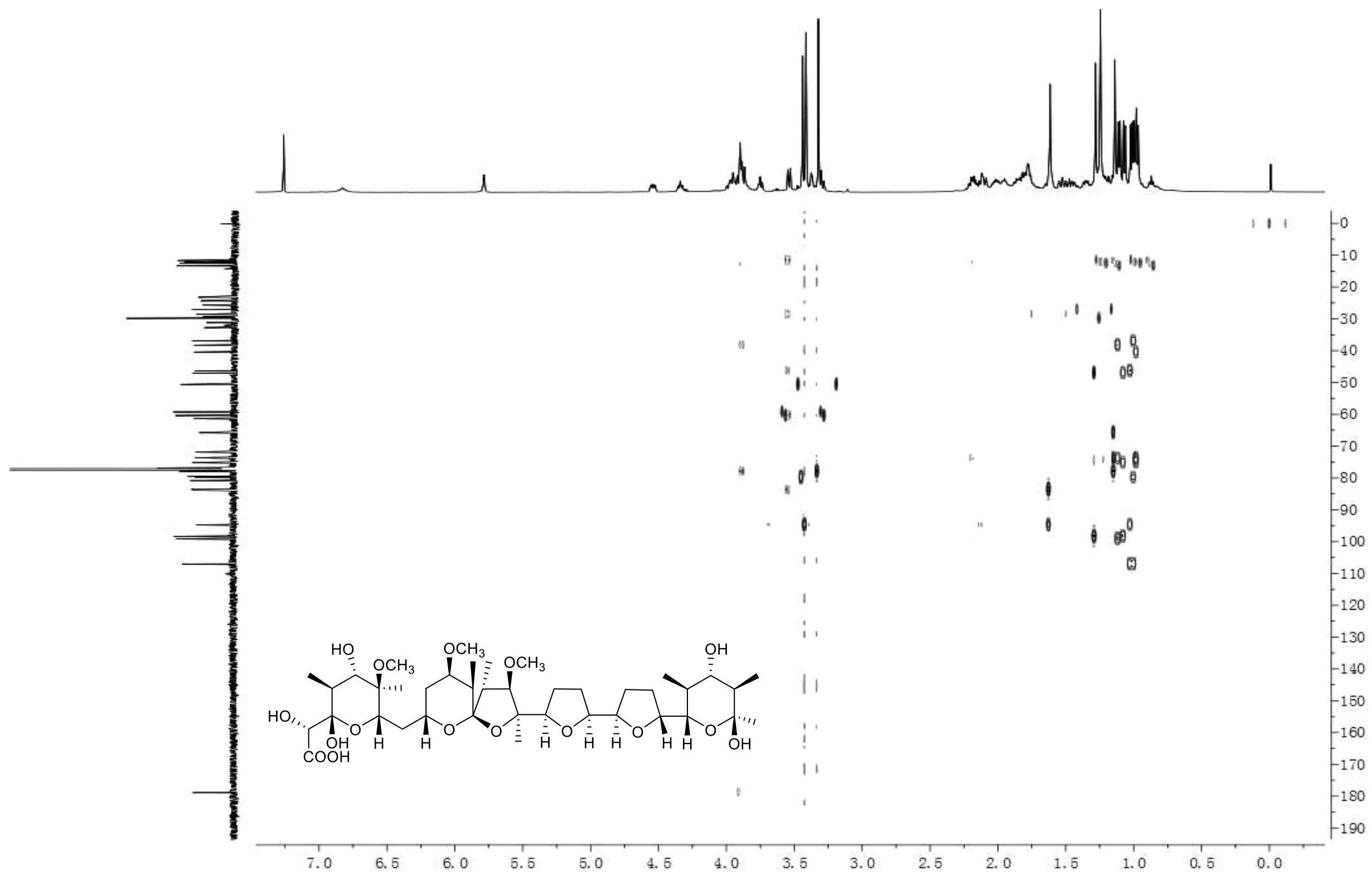


Figure S23. NOESY spectrum of compound $\mathbf{3}$ in $\mathrm{CDCl}_{3}$.

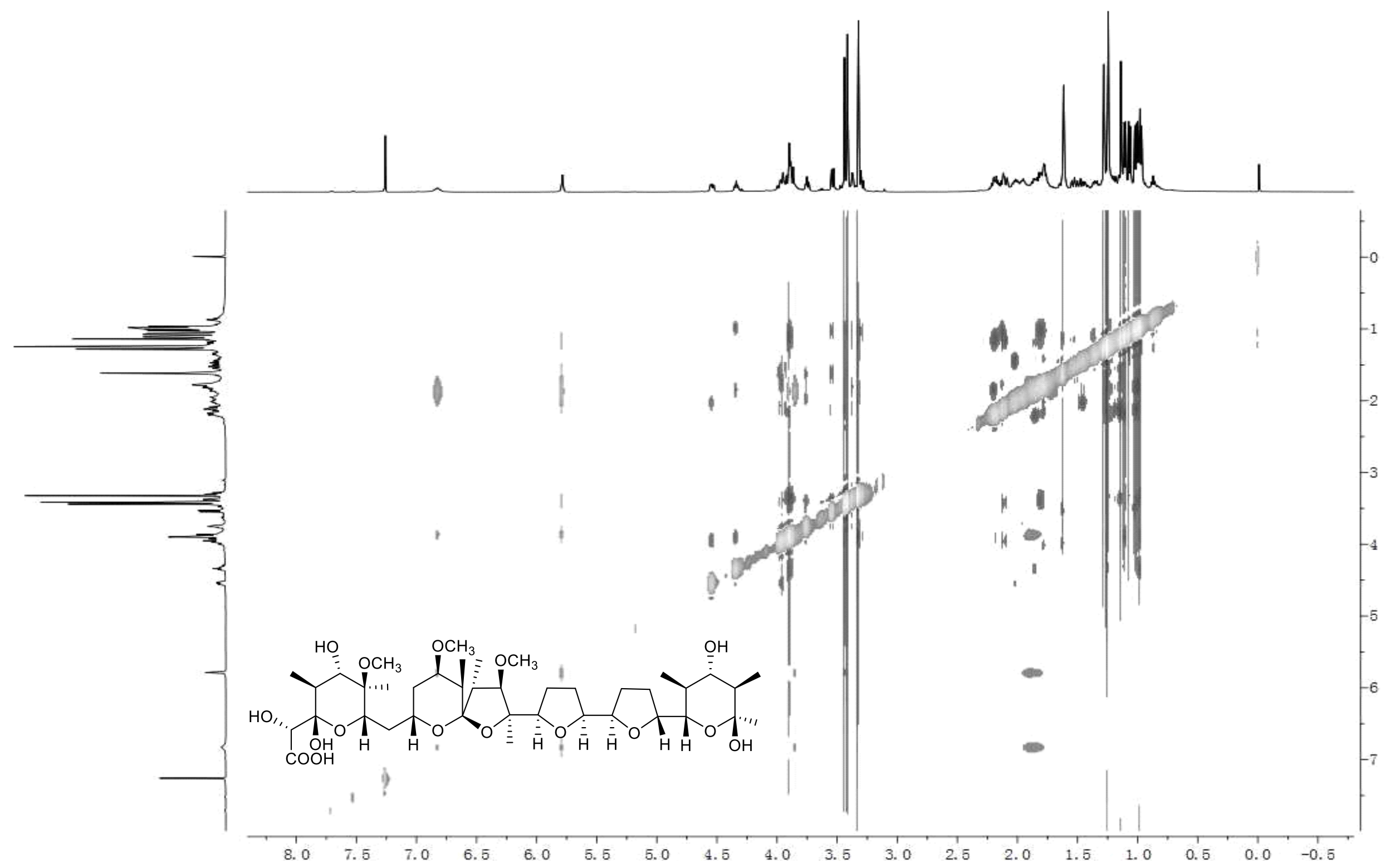


Figure S24. HR-ESI-MS spectrum of compound 4 in $\mathrm{MeOH}$.

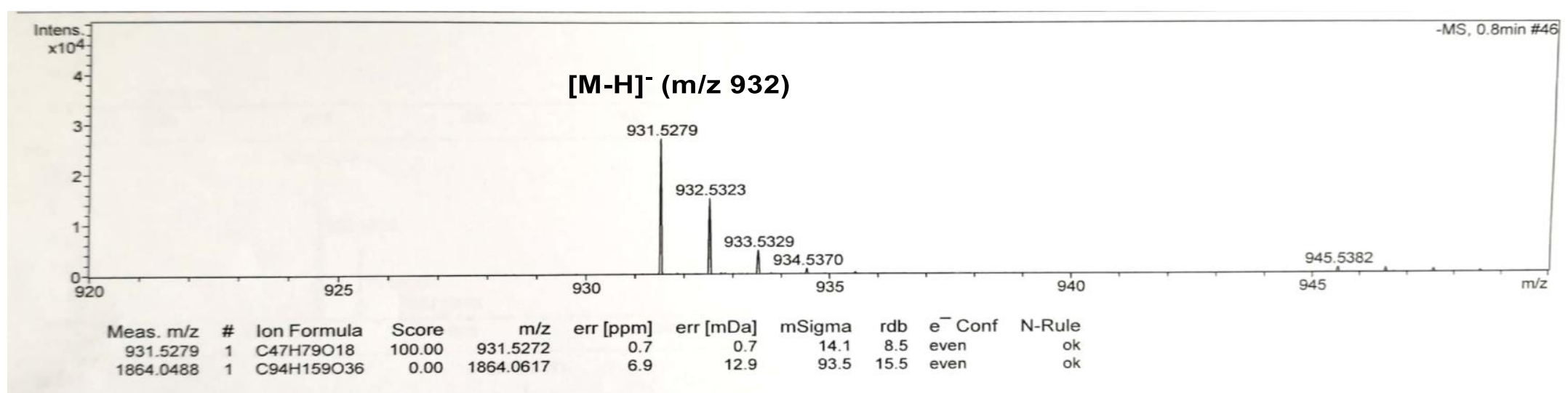

Figure S25. IR spectrum of compound 4.

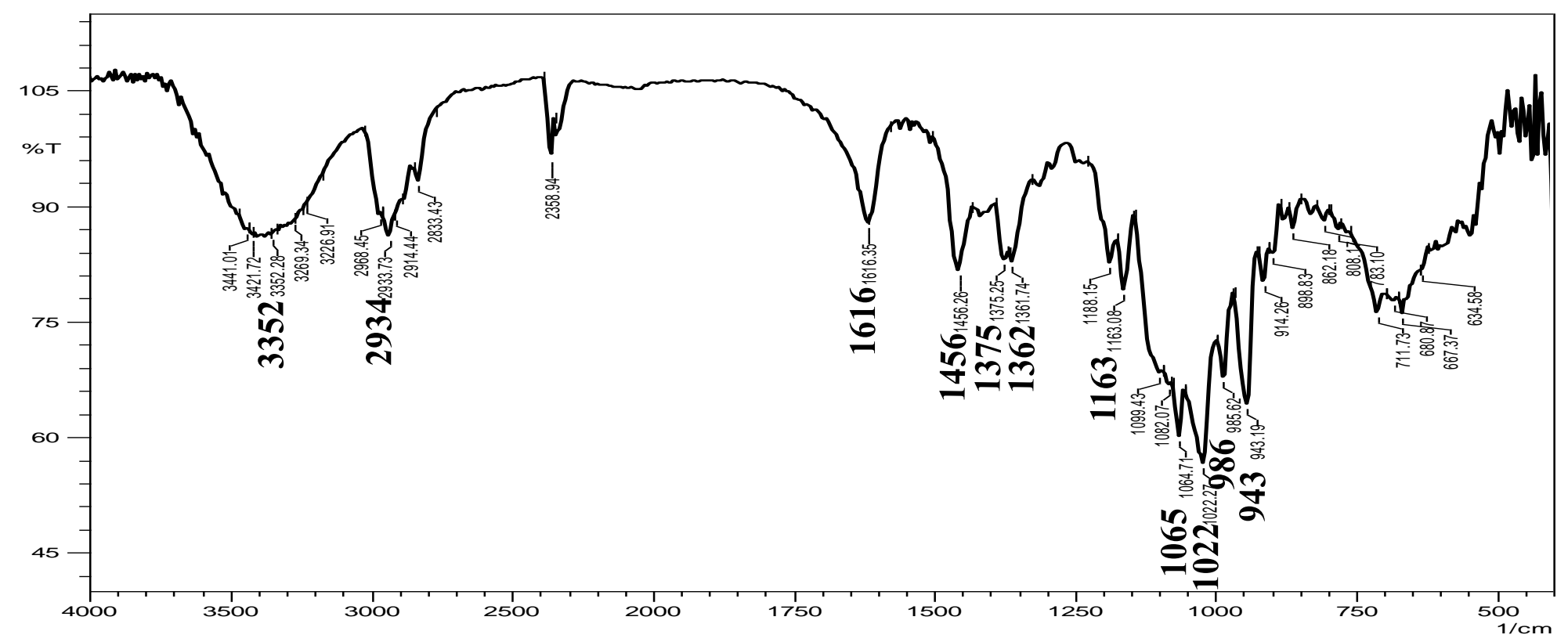


Figure S26. ${ }^{1} \mathrm{H}$ NMR $(500 \mathrm{MHz})$ spectrum of compound 4 in $\mathrm{CDCl}_{3}$.

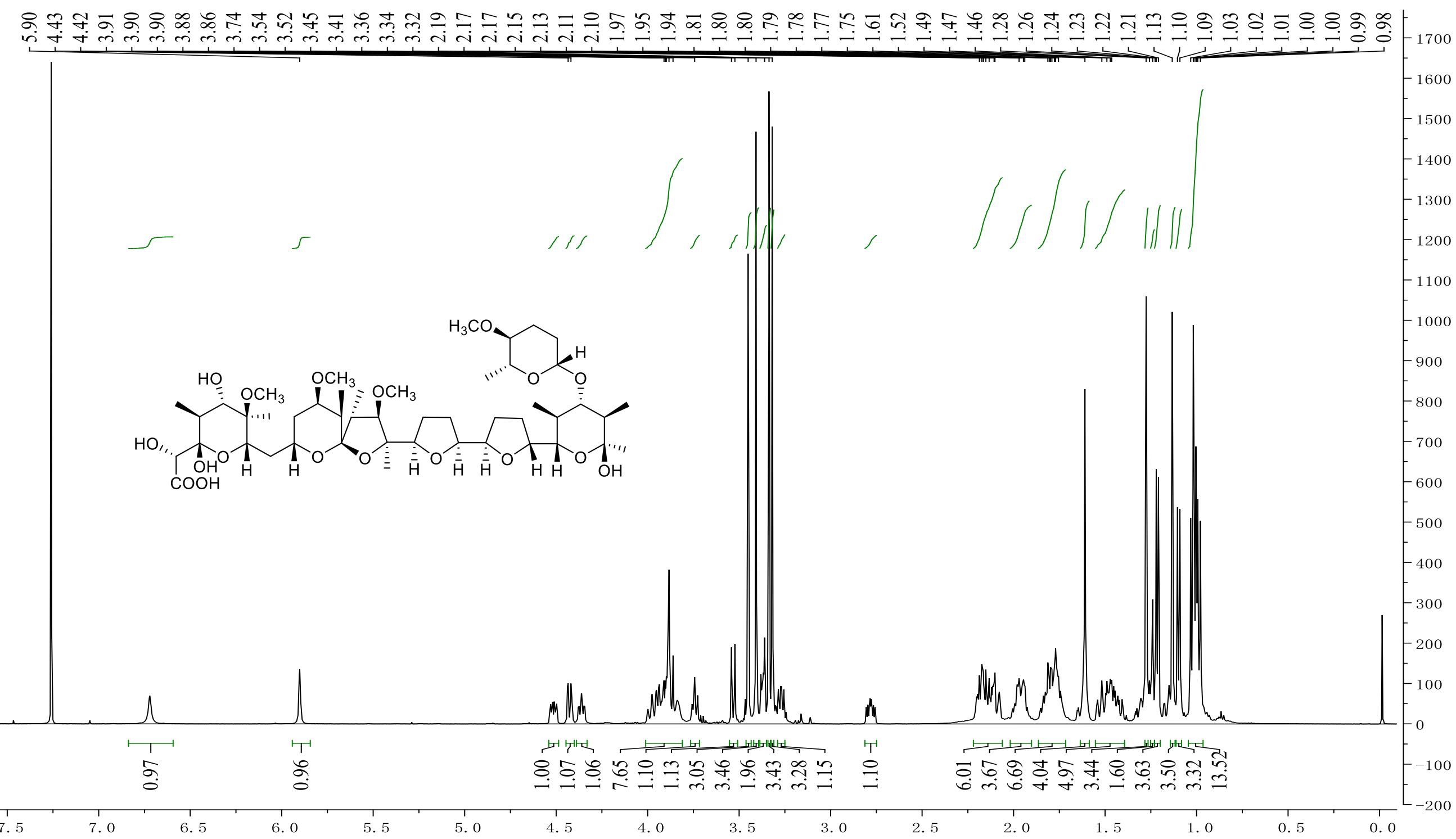


Figure S27. ${ }^{13} \mathrm{C}$ NMR $(125 \mathrm{MHz})$ spectrum of compound 4 in $\mathrm{CDCl}_{3}$.

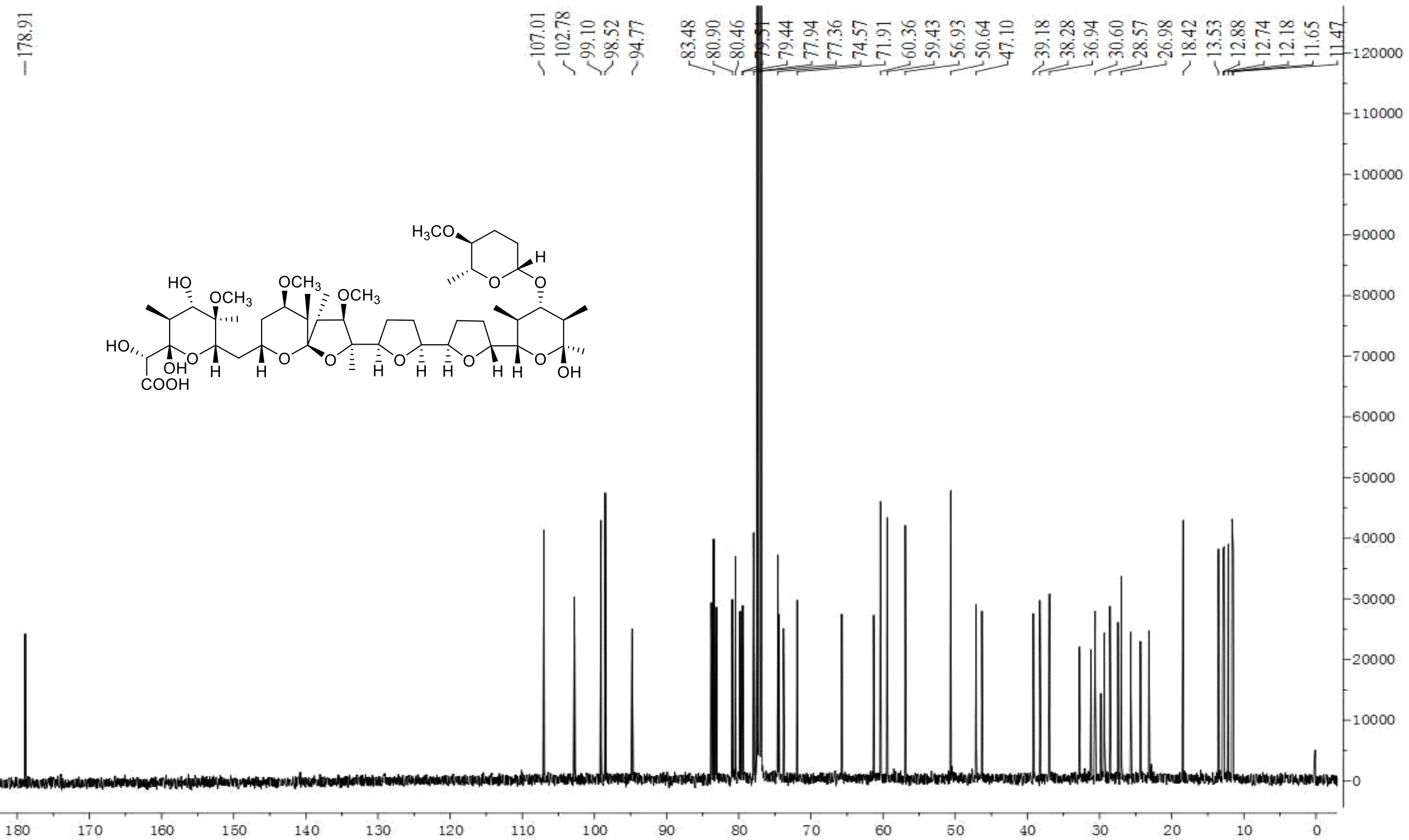


Figure S28. ${ }^{13} \mathrm{C}$ DEPT spectrum of compound 4 in $\mathrm{CDCl}_{3}$.

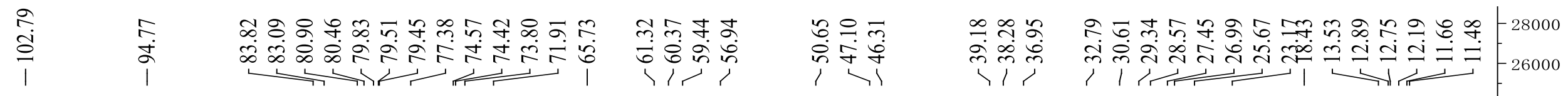

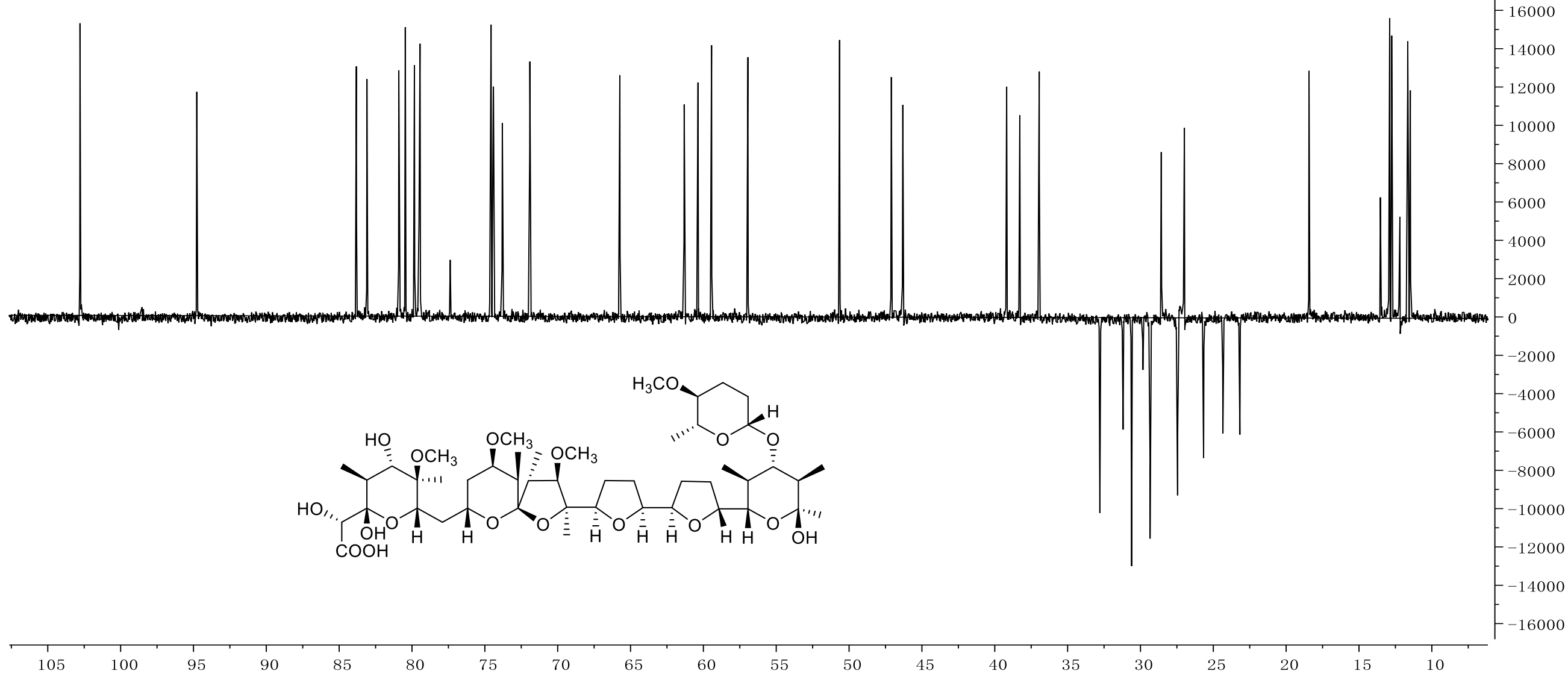


Figure S29. ${ }^{1} \mathrm{H}-{ }^{1} \mathrm{H}$ COSY spectrum of compound 4 in $\mathrm{CDCl}_{3}$.

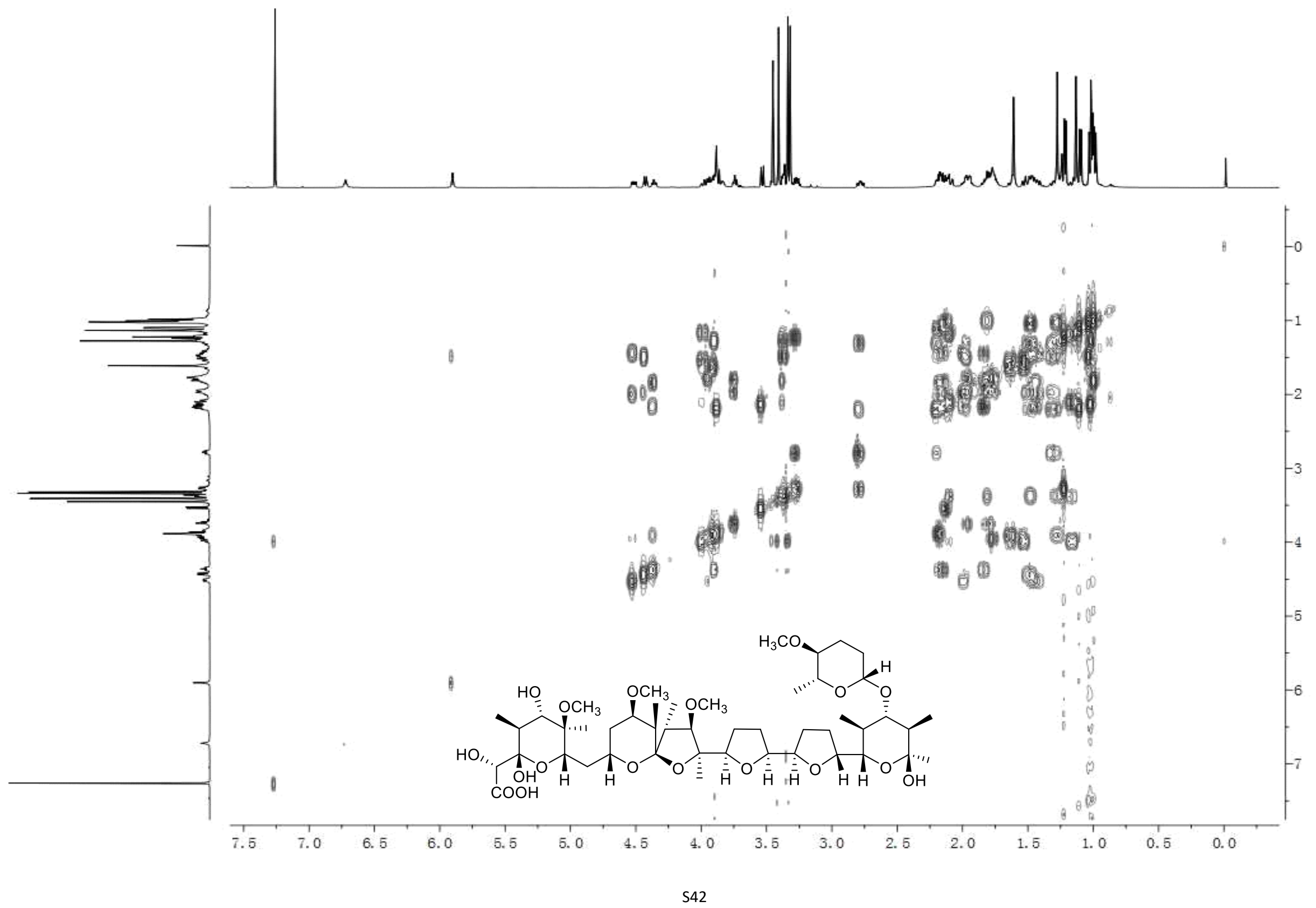


Figure S30. HSQC spectrum of compound 4 in $\mathrm{CDCl}_{3}$.

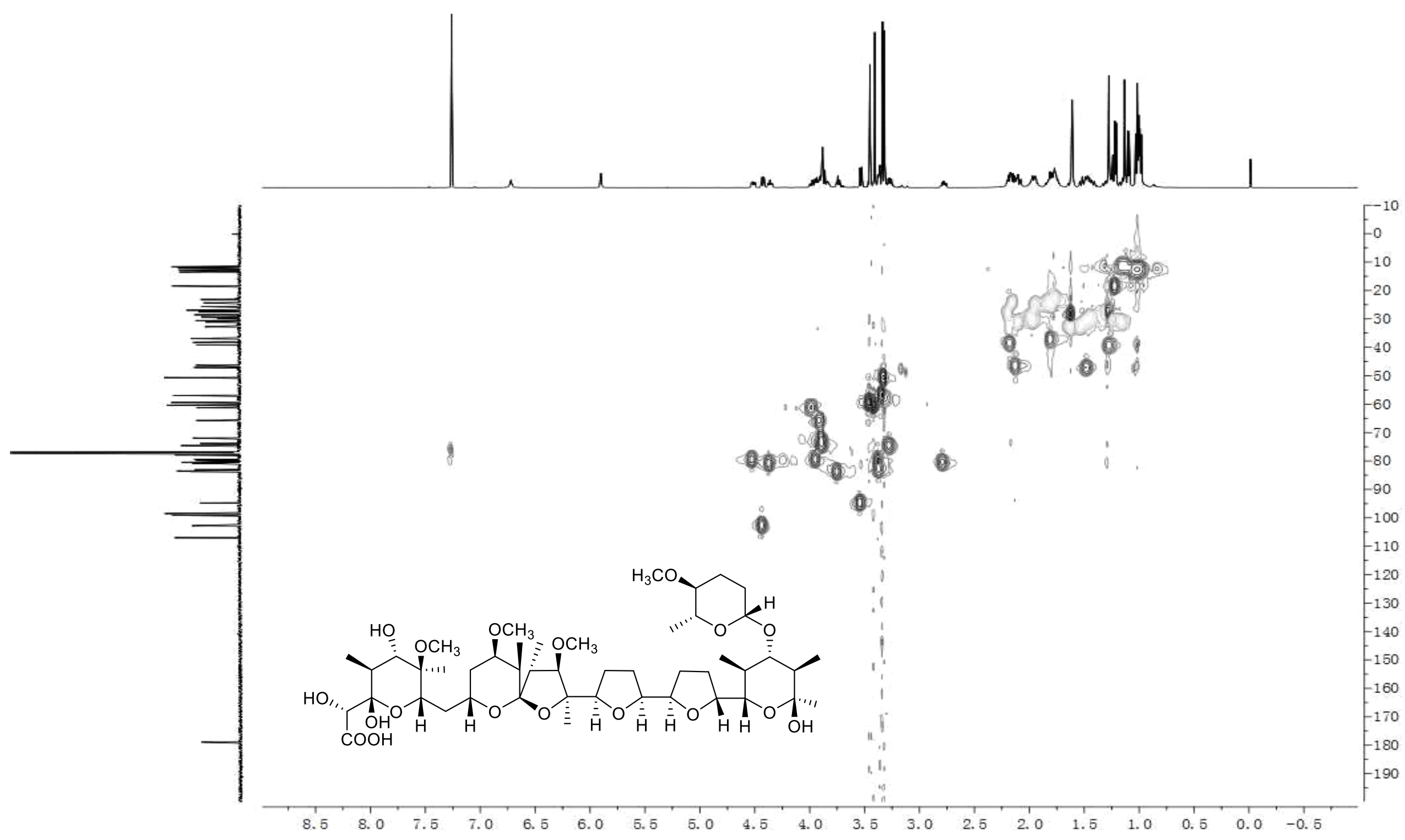


Figure S31. HMBC spectrum of compound 4 in $\mathrm{CDCl}_{3}$.




Figure S32. NOESY spectrum of compound 4 in $\mathrm{CDCl}_{3}$.

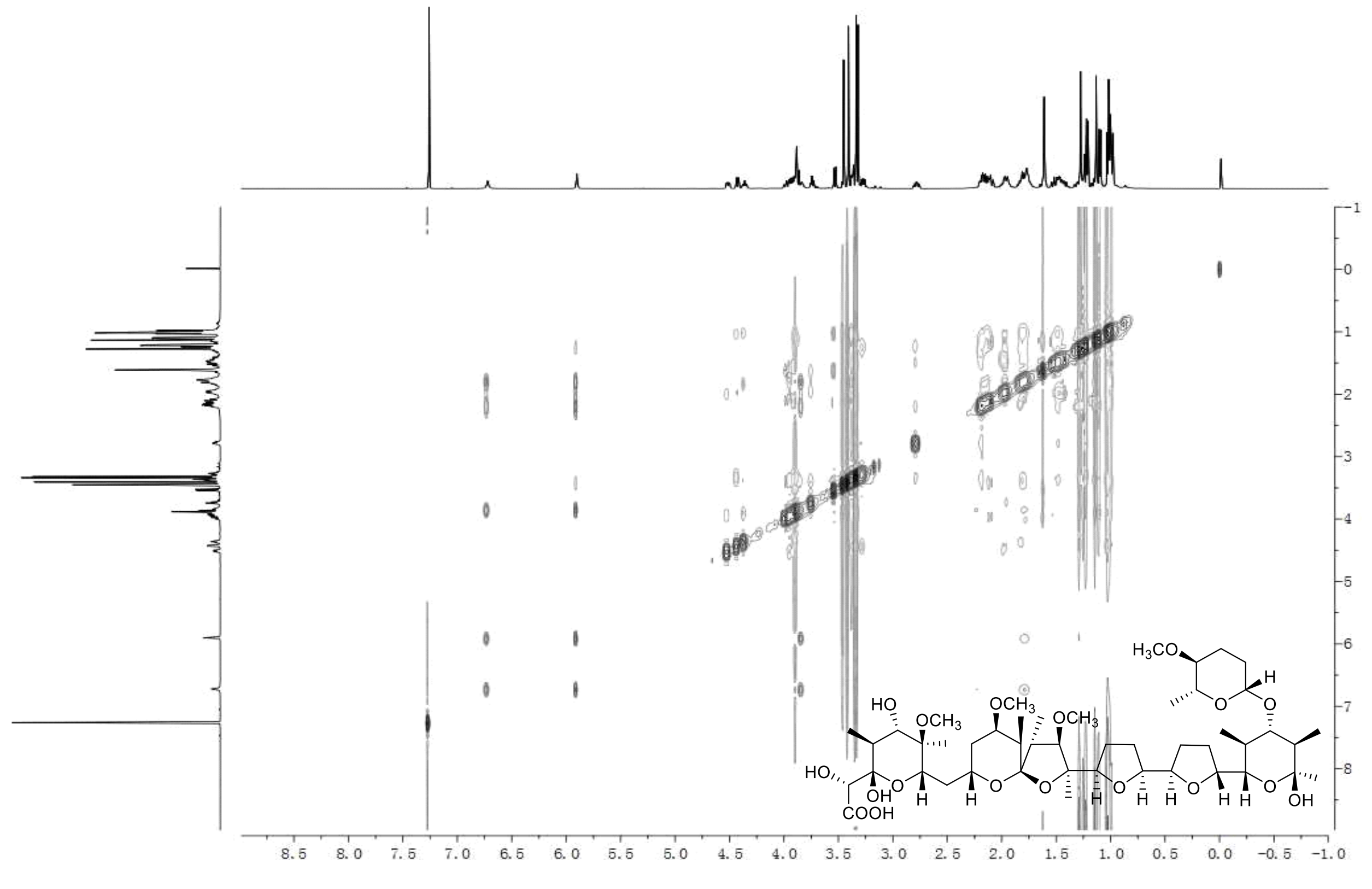


Figure S33. HR-ESI-MS spectrum of compound 5 in $\mathrm{MeOH}$.

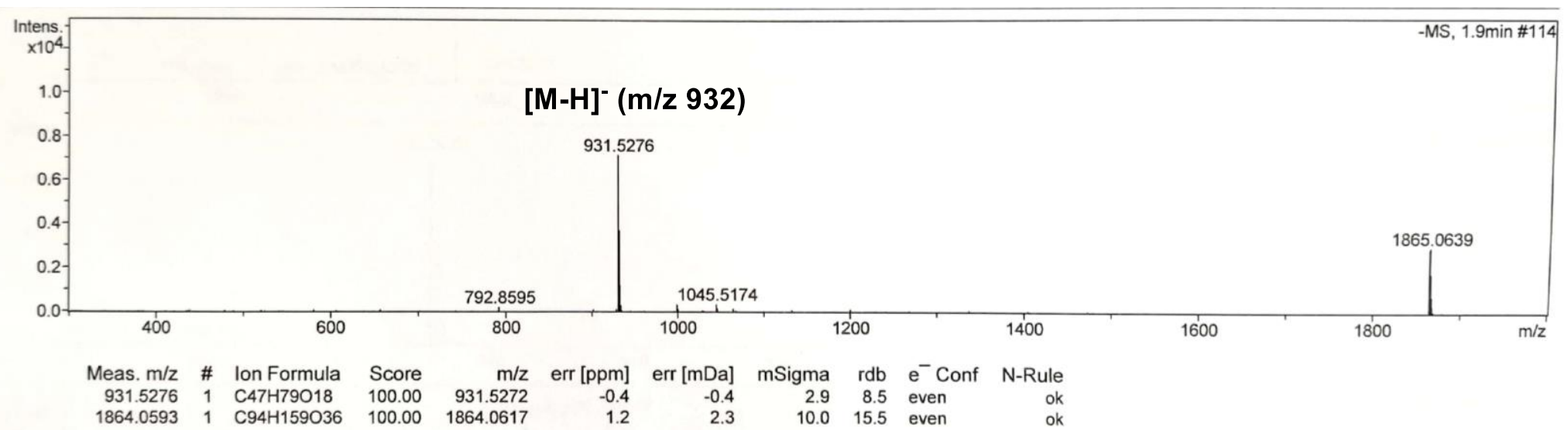

Figure S34. IR spectrum of compound 5.

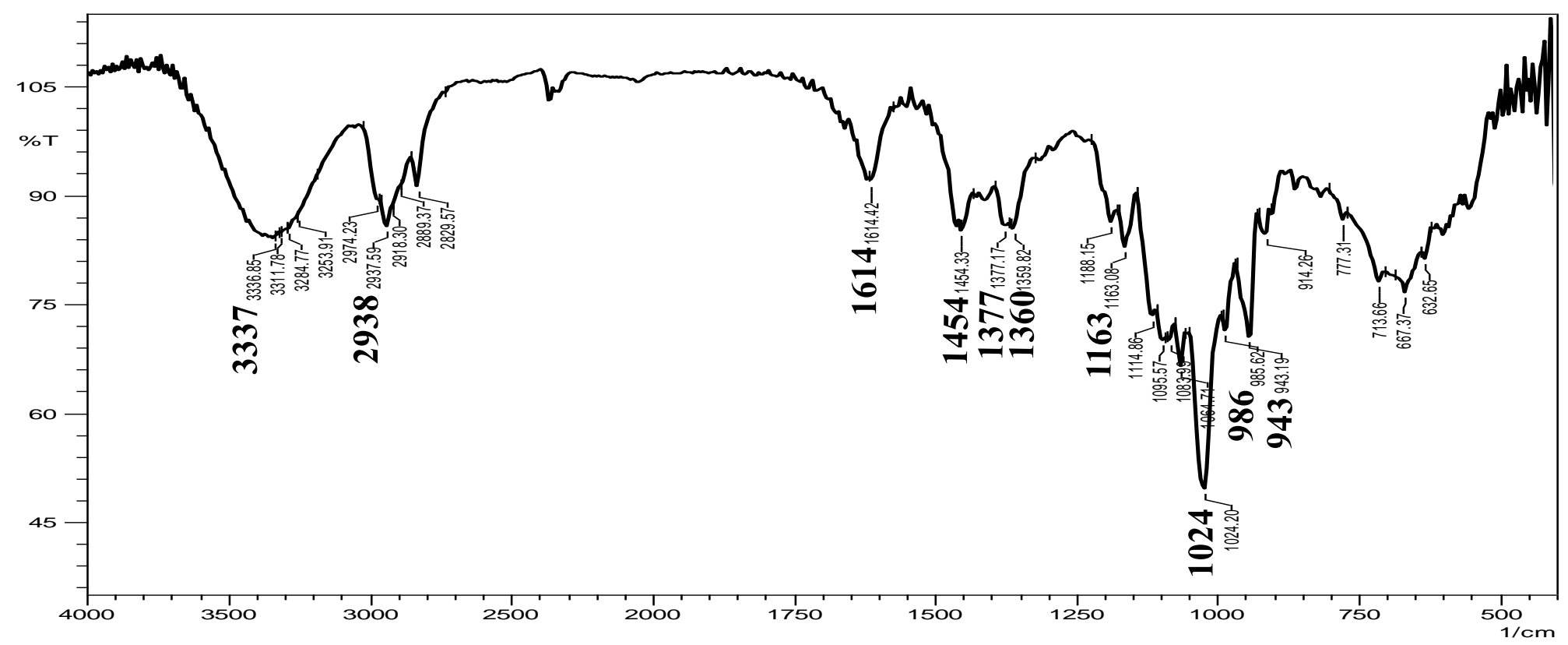


Figure S35. ${ }^{1} \mathrm{H}$ NMR $(700 \mathrm{MHz})$ spectrum of compound 5 in $\mathrm{CDCl}_{3}$.

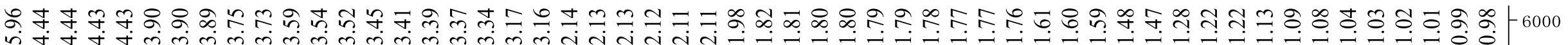

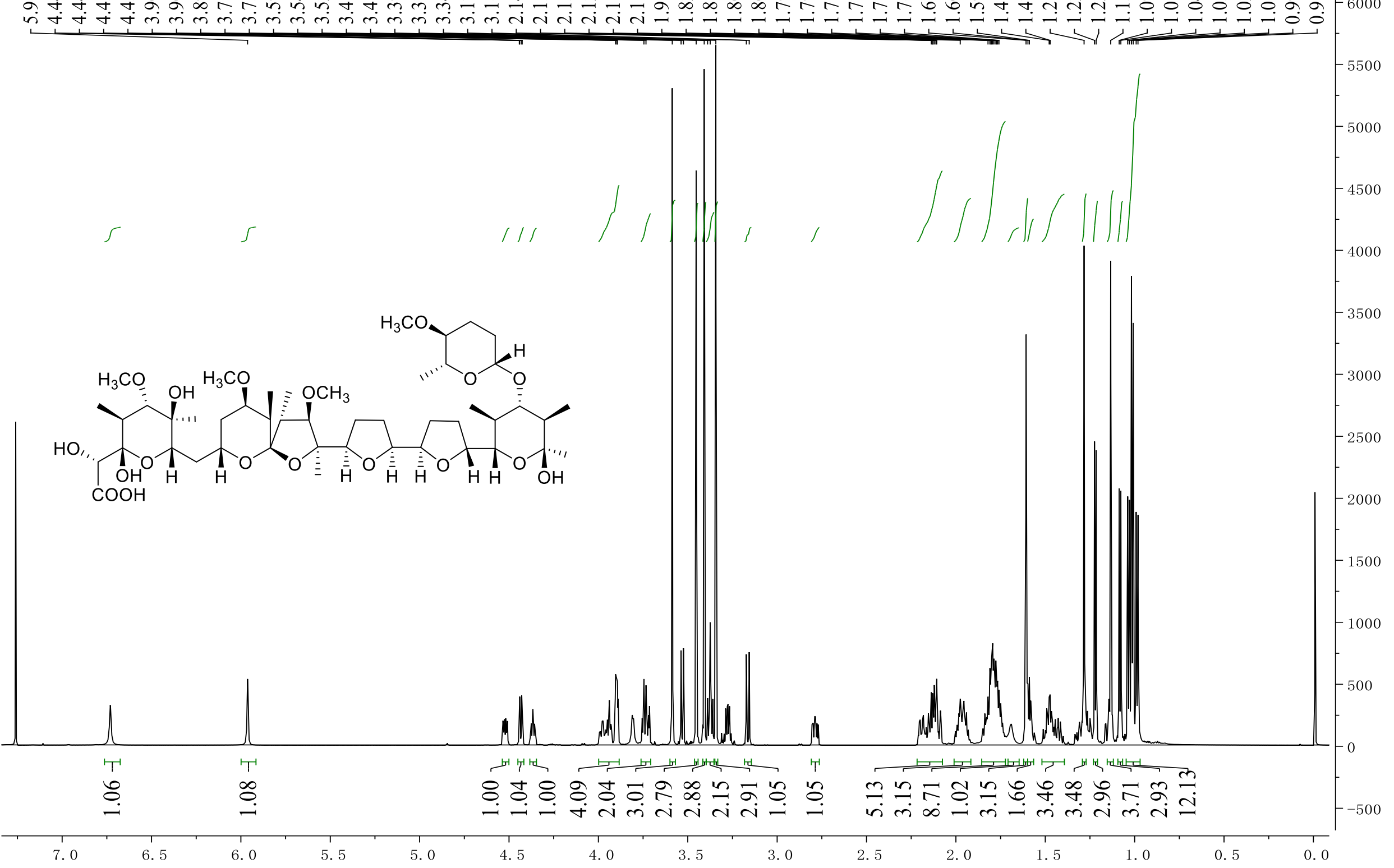


Figure S36. ${ }^{13} \mathrm{C}$ NMR $(175 \mathrm{MHz})$ spectrum of compound 5 in $\mathrm{CDCl}_{3}$.

$\stackrel{5}{\frac{2}{2}}$

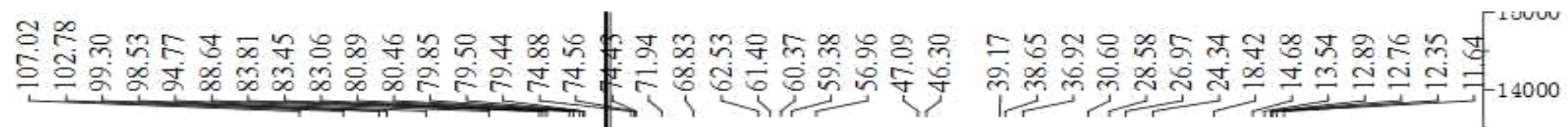
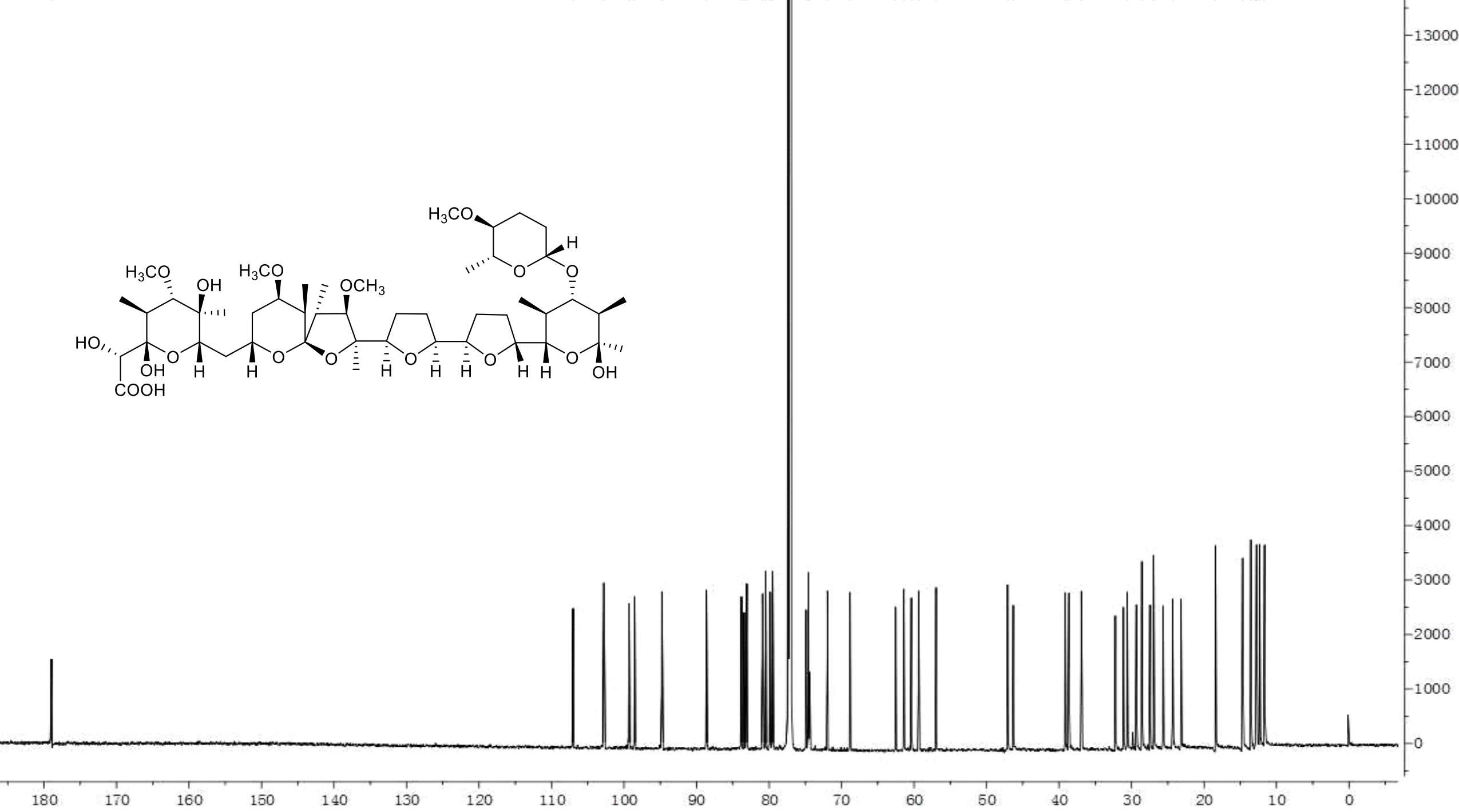
Figure S37. ${ }^{13} \mathrm{C}$ DEPT spectrum of compound $\mathbf{5}$ in $\mathrm{CDCl}_{3}$.

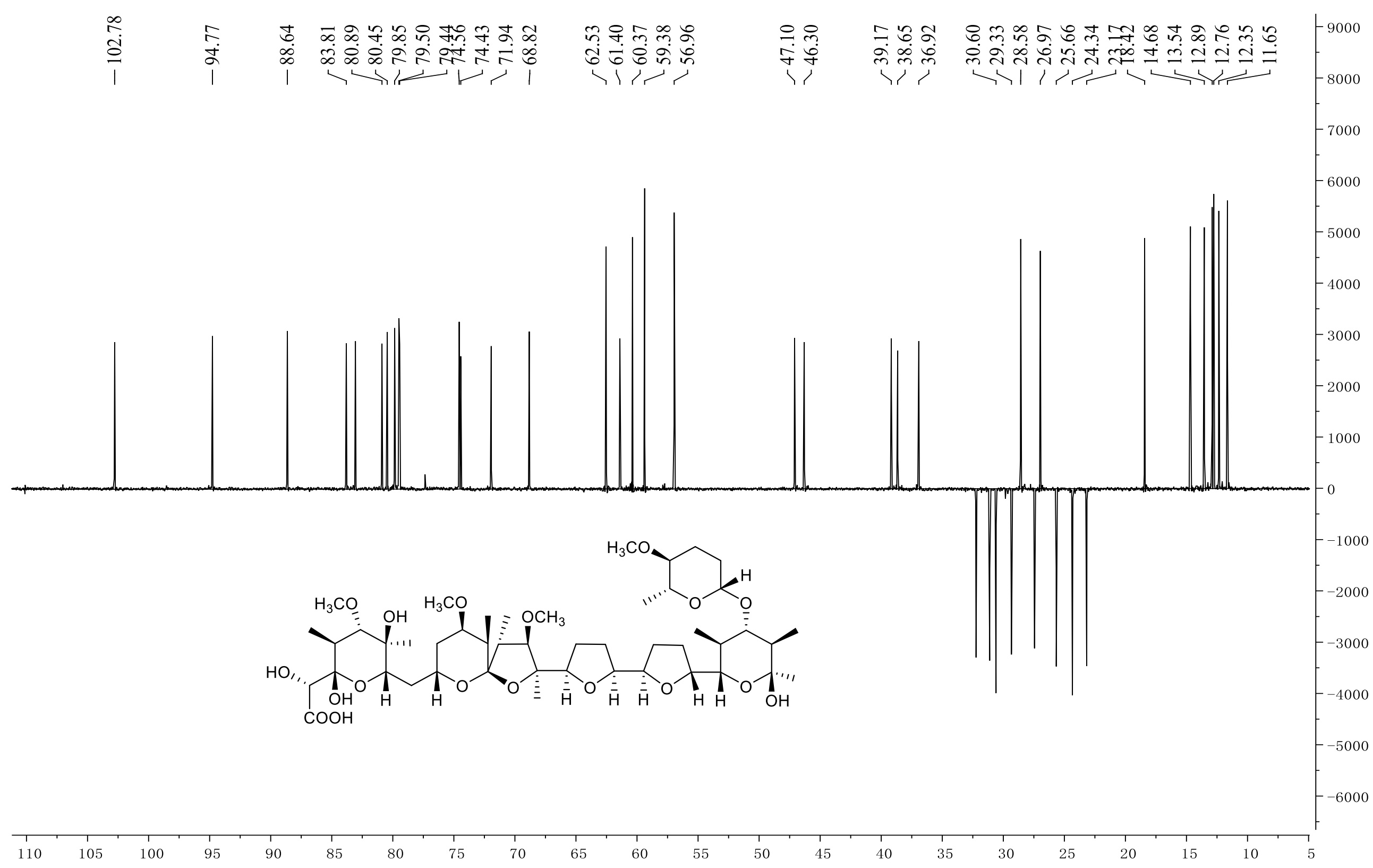


Figure S38. ${ }^{1} \mathrm{H}-{ }^{1} \mathrm{H}$ COSY spectrum of compound 5 in $\mathrm{CDCl}_{3}$.

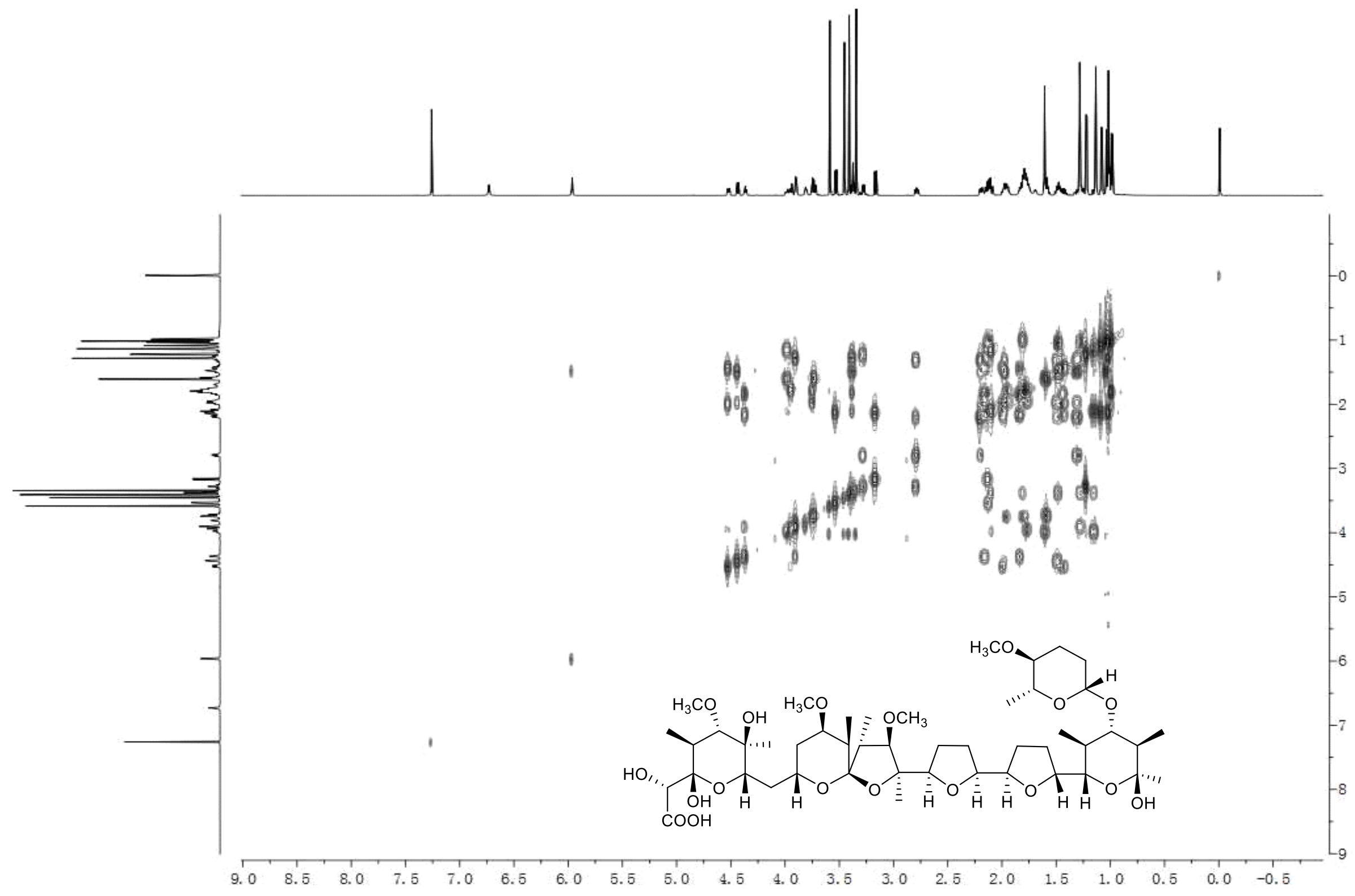


Figure S39. HSQC spectrum of compound 5 in $\mathrm{CDCl}_{3}$.

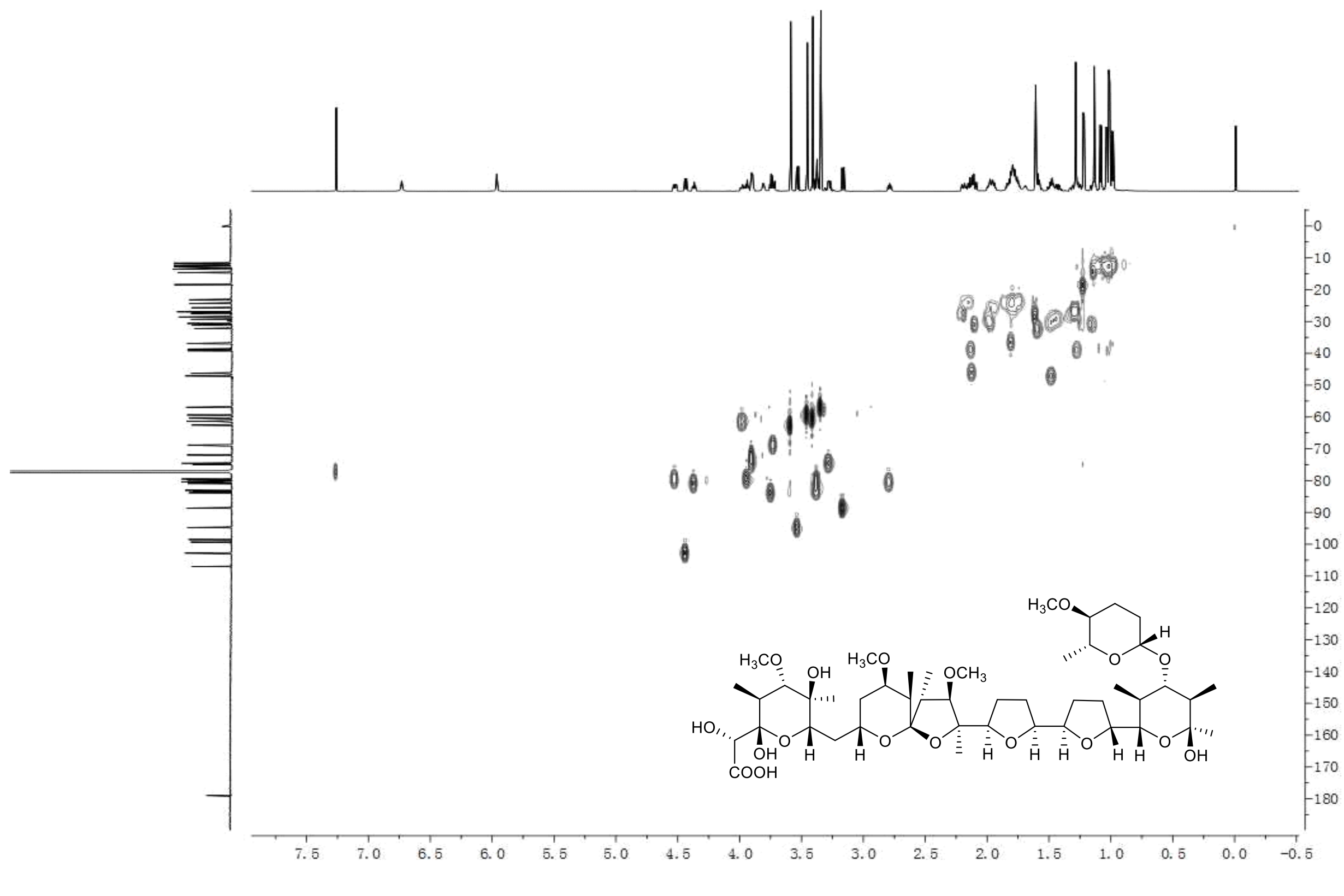


Figure S40. HMBC spectrum of compound $\mathbf{5}$ in $\mathrm{CDCl}_{3}$.

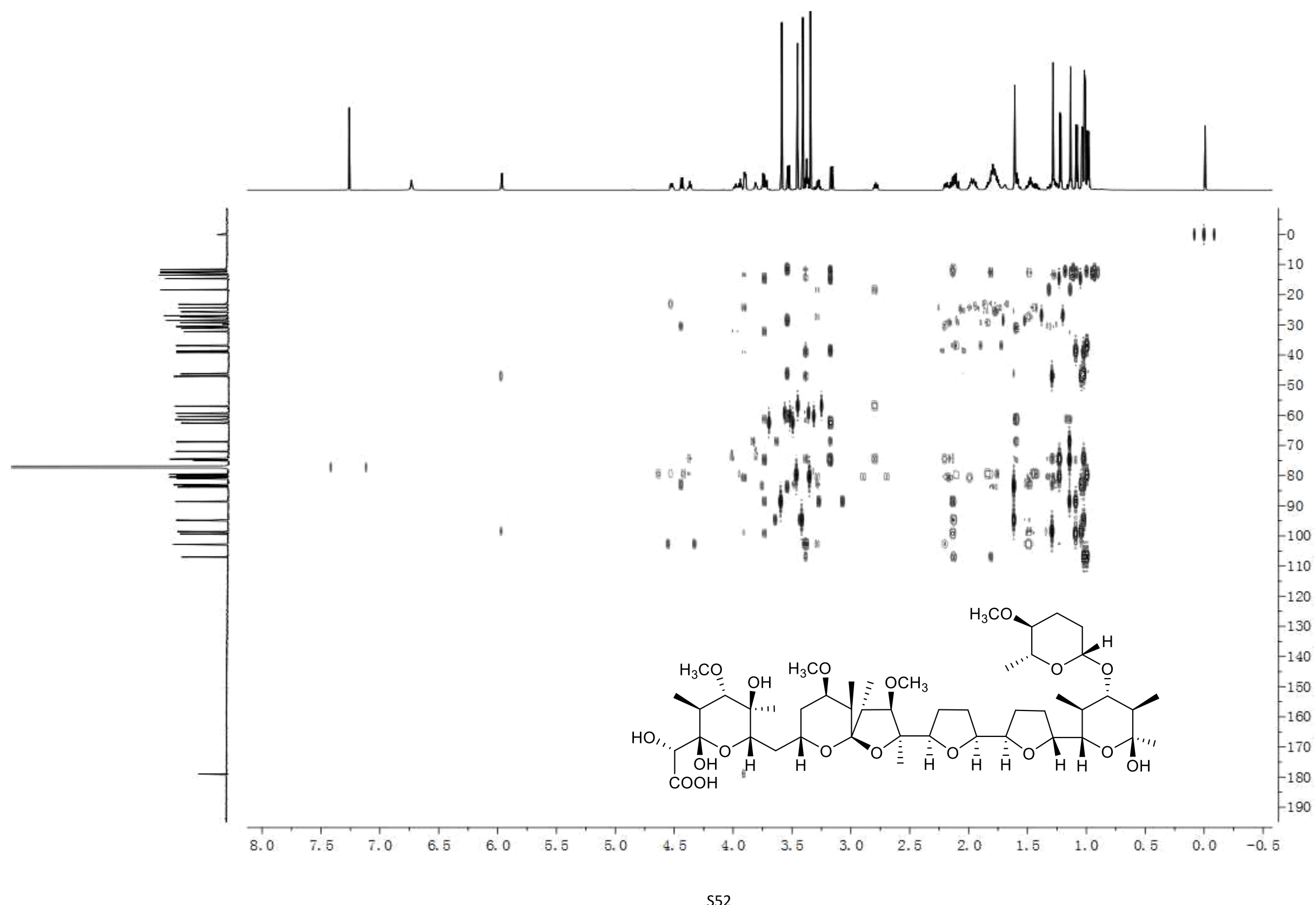


Figure S41. NOESY spectrum of compound 5 in $\mathrm{CDCl}_{3}$.

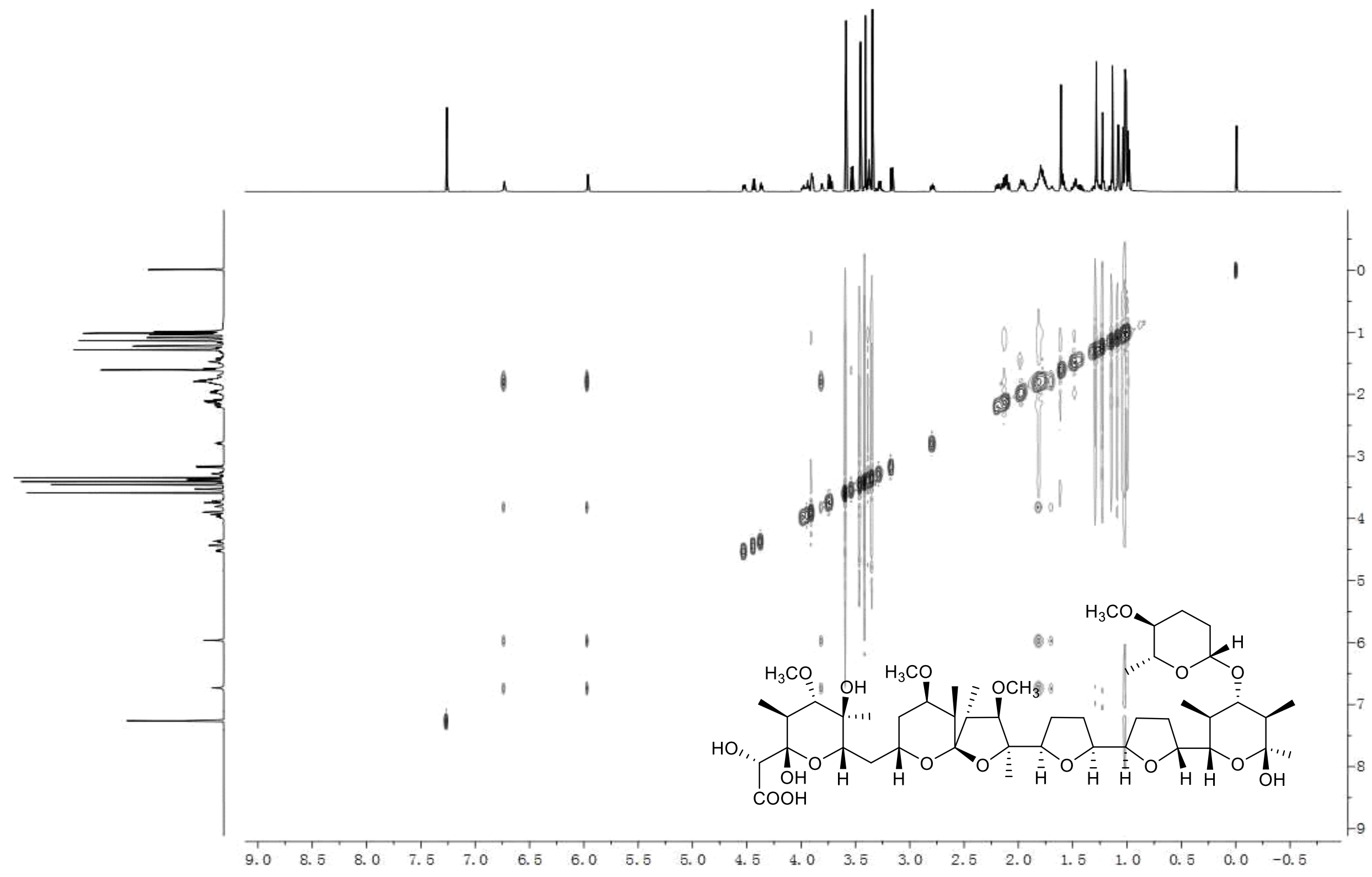


Figure S42. HR-ESI-MS spectrum of compound 6 in $\mathrm{MeOH}$.

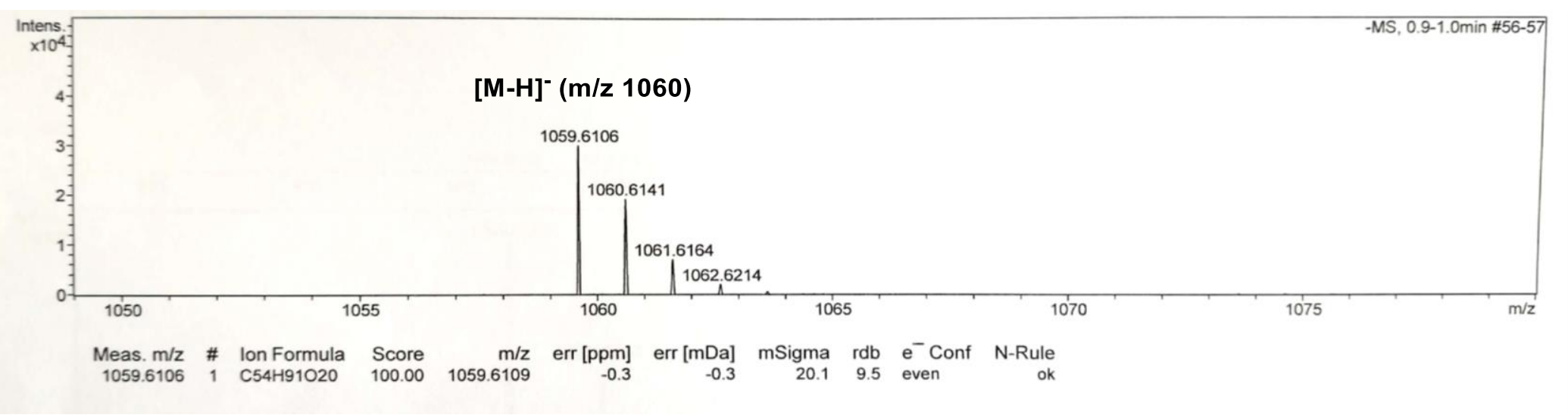

Figure S43. IR spectrum of compound 6.

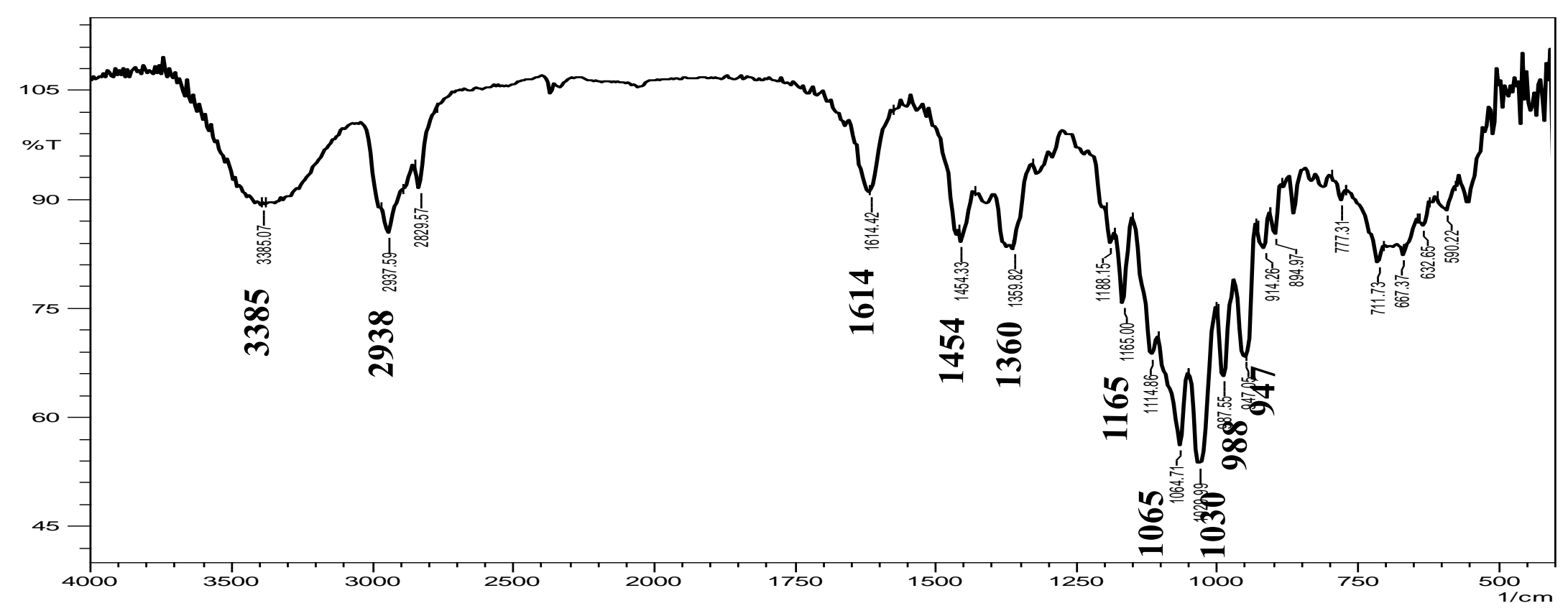


Figure S44. ${ }^{1} \mathrm{H}$ NMR $(700 \mathrm{MHz})$ spectrum of compound 6 in $\mathrm{CDCl}_{3}$.

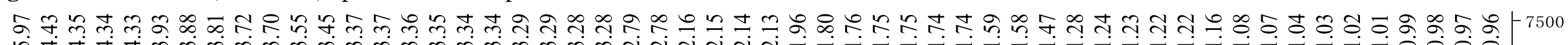

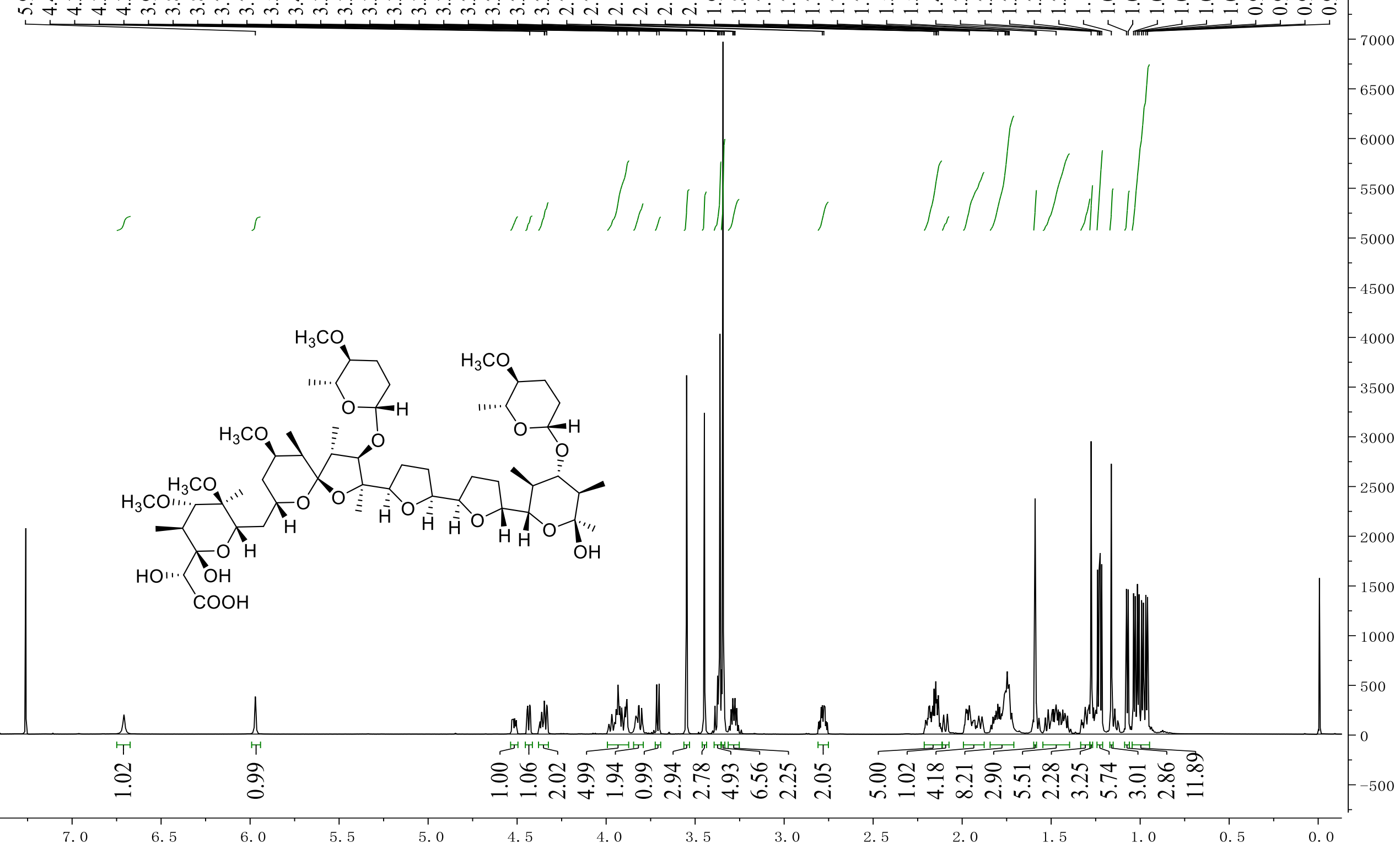


Figure S45. ${ }^{13} \mathrm{C}$ NMR $(175 \mathrm{MHz})$ spectrum of compound 6 in $\mathrm{CDCl}_{3}$.

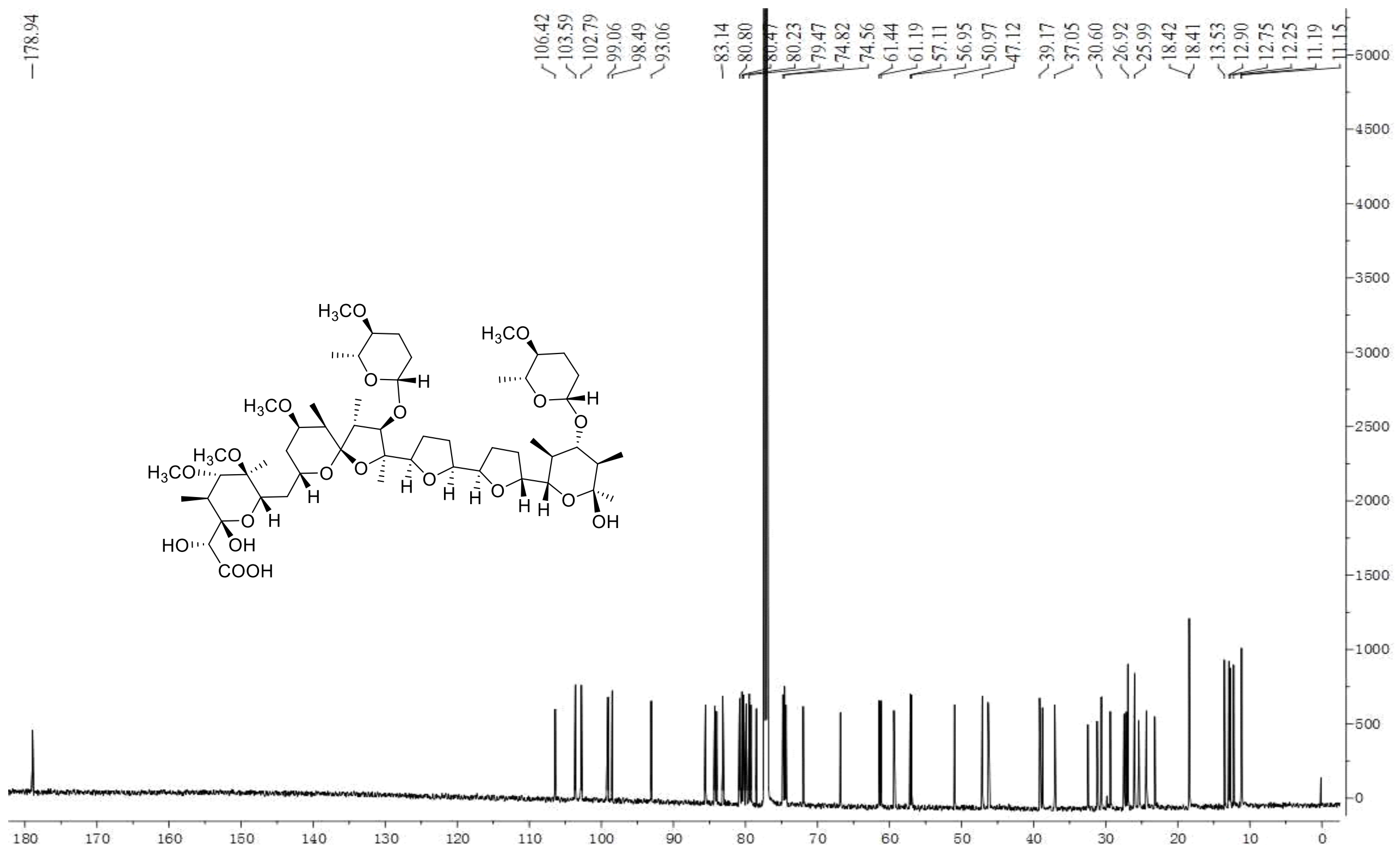


Figure S46. ${ }^{13} \mathrm{C}$ DEPT spectrum of compound 6 in $\mathrm{CDCl}_{3}$.

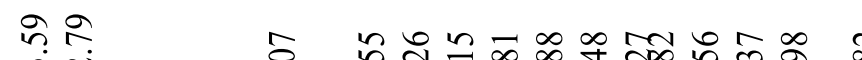

ฮิ

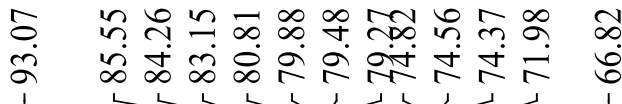

Ұㅇำ

ลิำ ซึ่

I $\infty 8$

लें

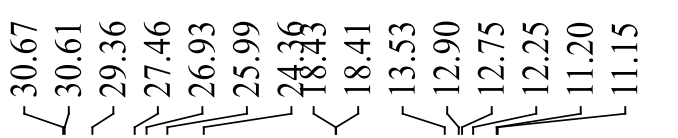

$-1500$

$1<1$

।?

1300

1200

1200

1000
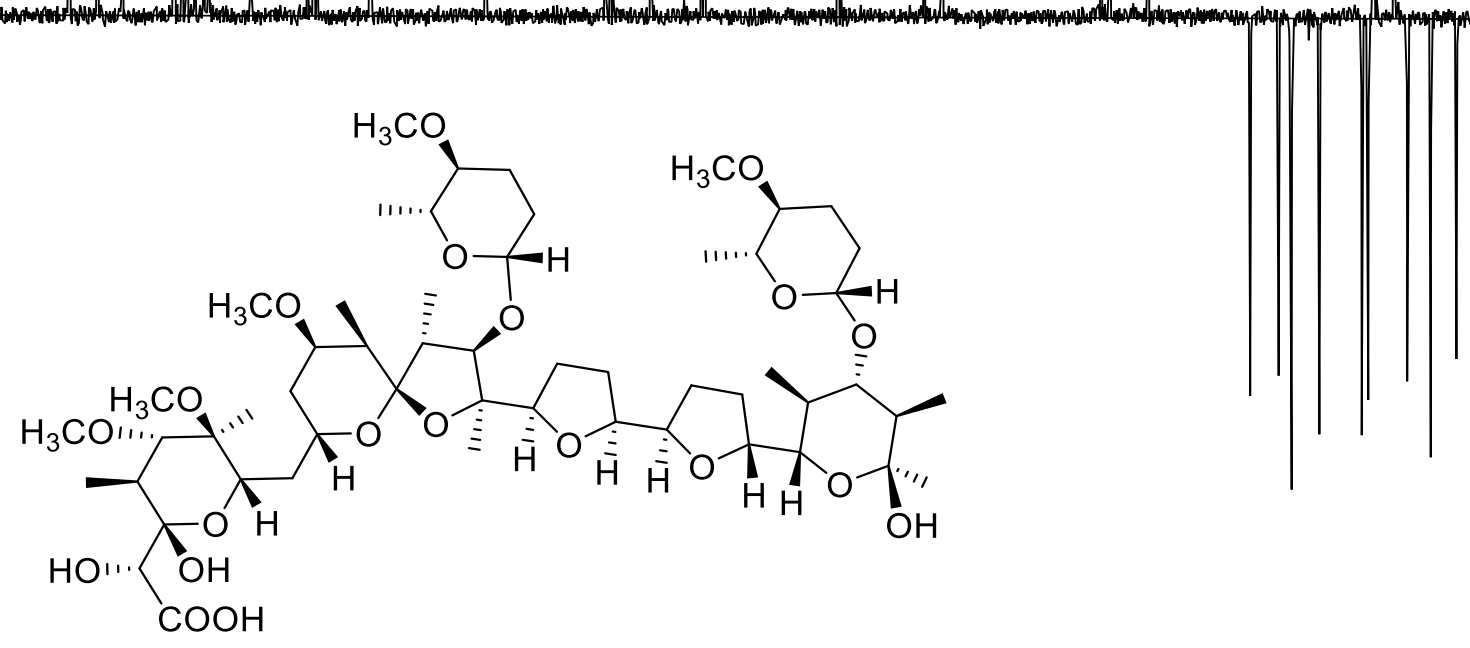
Figure S47. ${ }^{1} \mathrm{H}-{ }^{1} \mathrm{H}$ COSY spectrum of compound 6 in $\mathrm{CDCl}_{3}$.

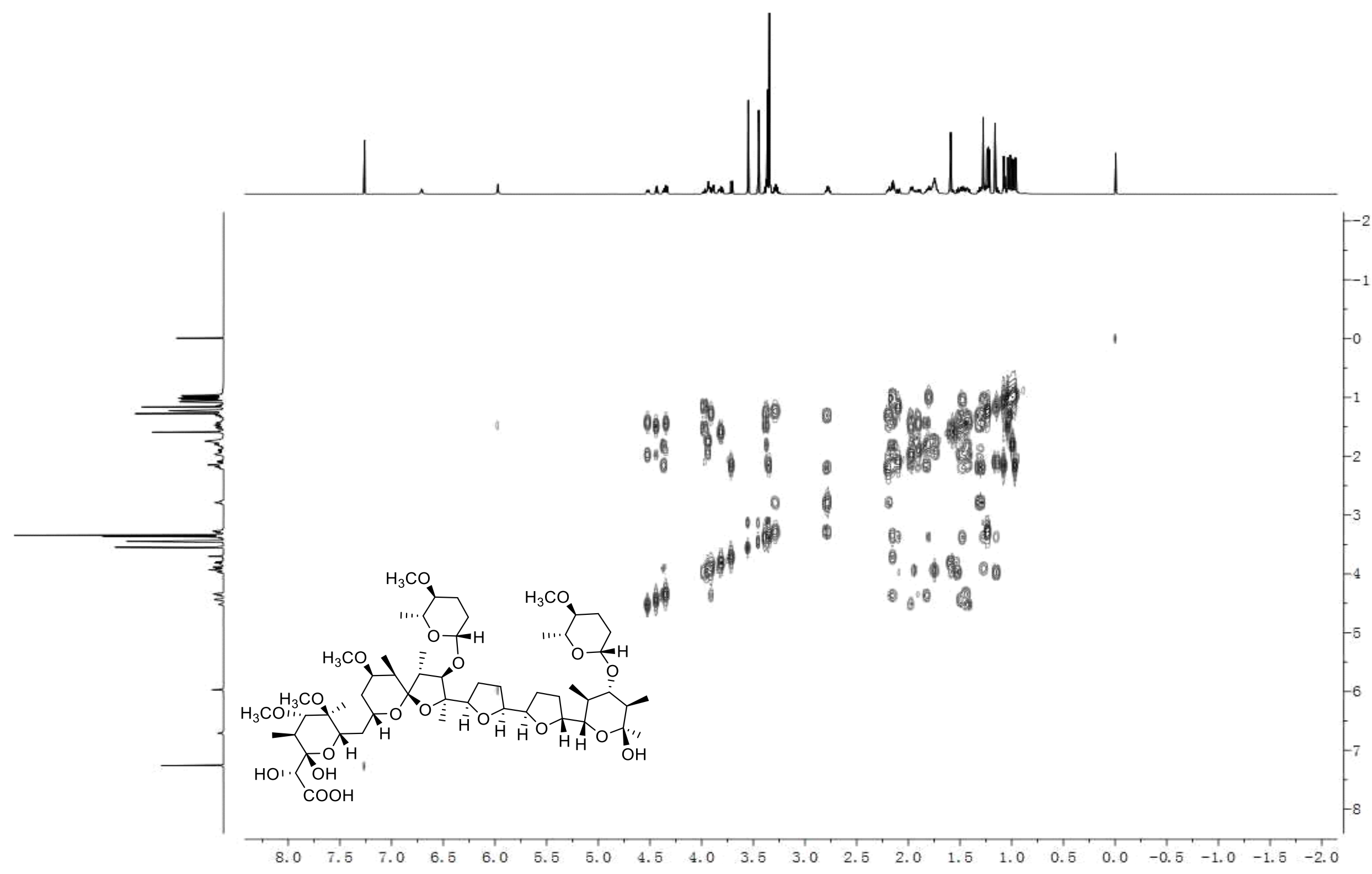


Figure S48. HSQC spectrum of compound 6 in $\mathrm{CDCl}_{3}$.

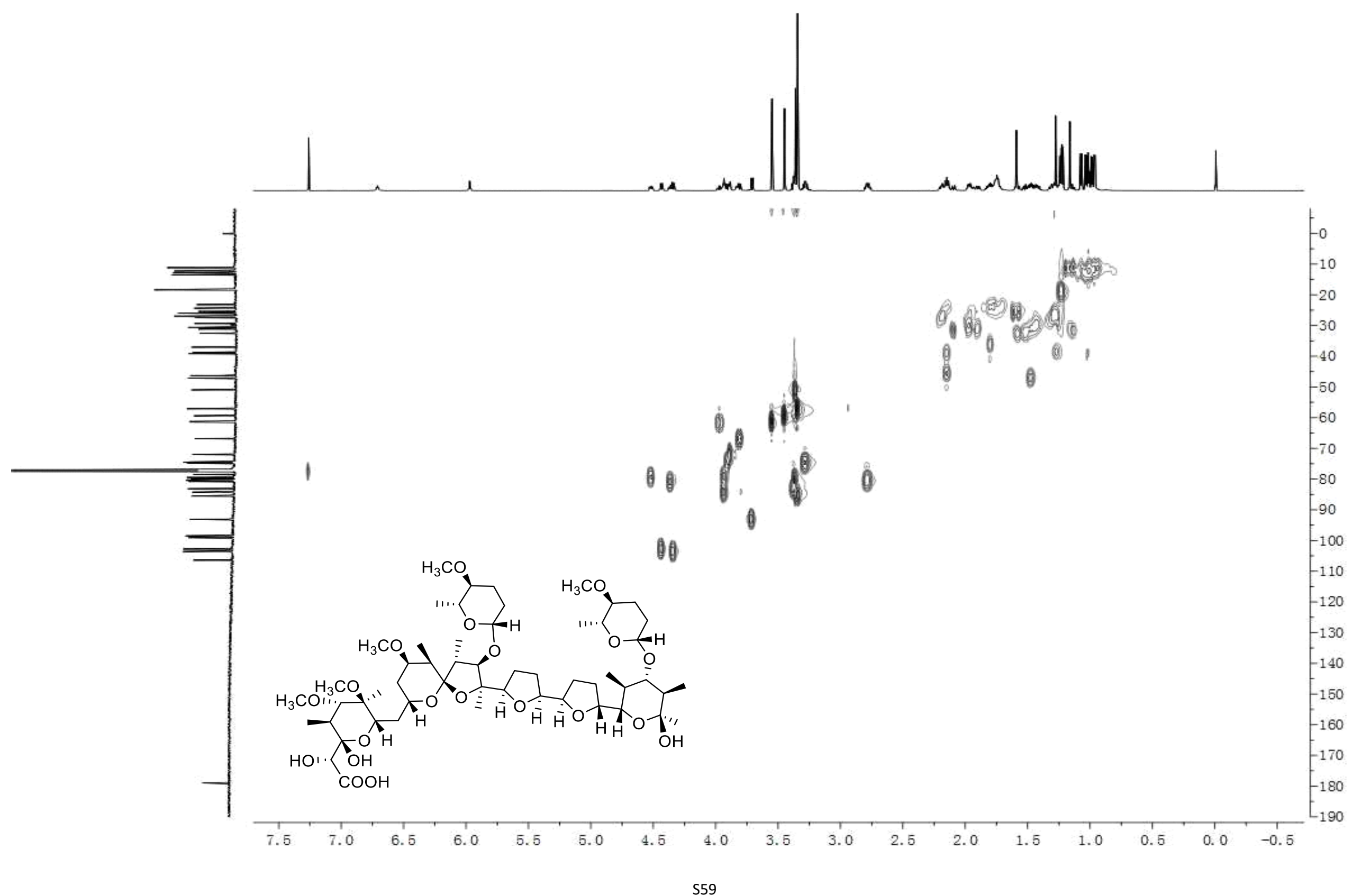


Figure S49. HMBC spectrum of compound 6 in $\mathrm{CDCl}_{3}$.

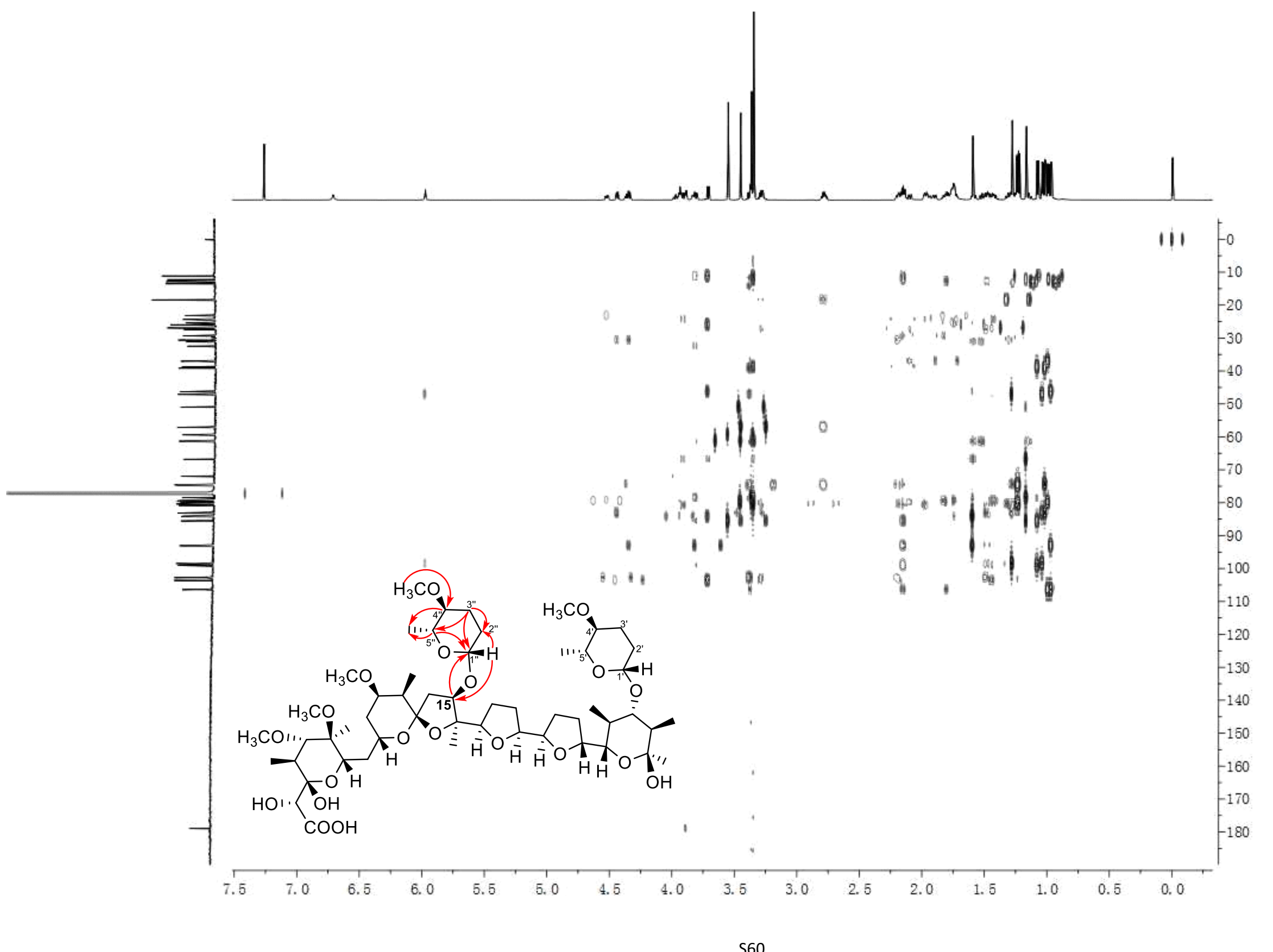


Figure S50. NOESY spectrum of compound 6 in $\mathrm{CDCl}_{3}$.

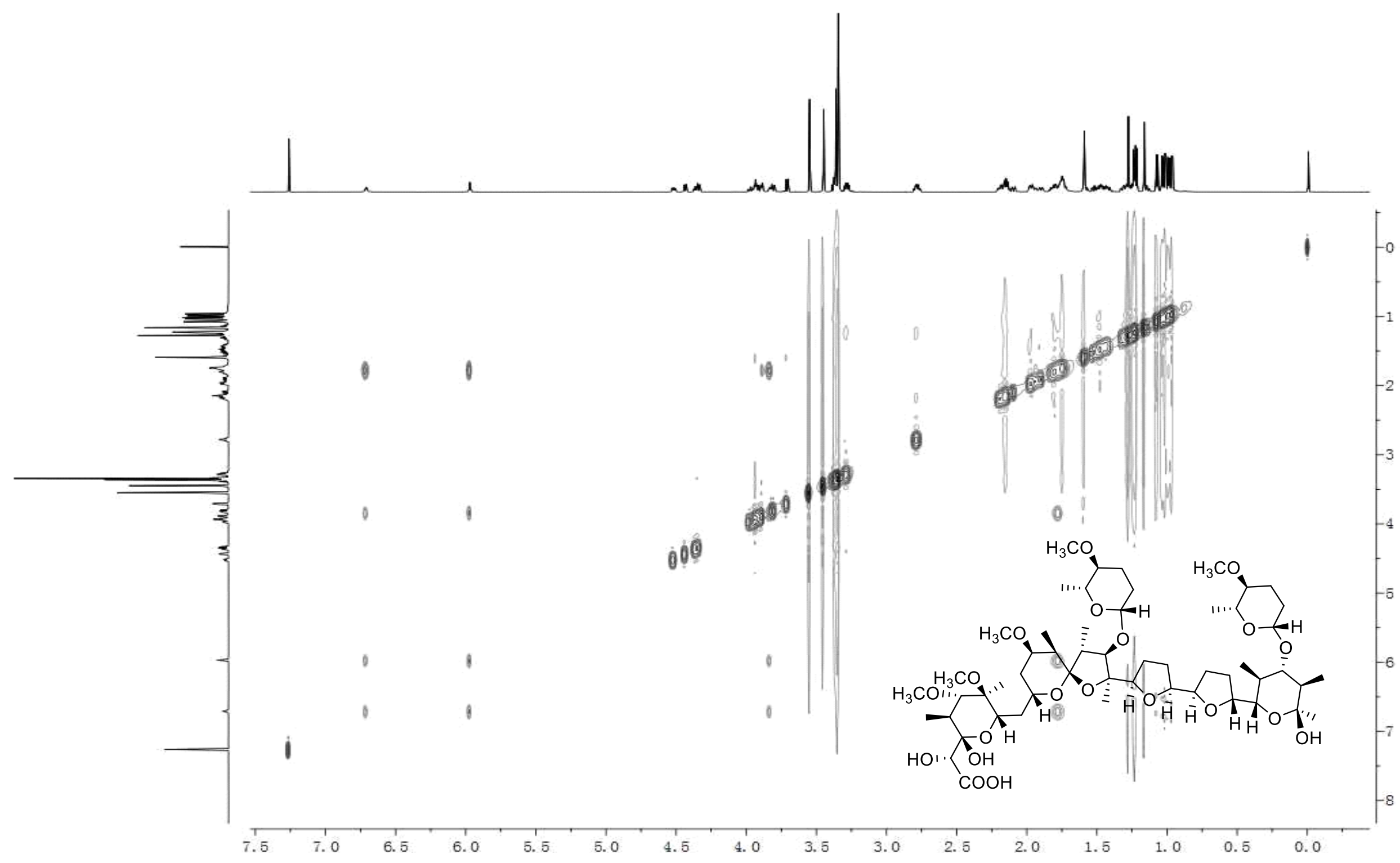


Figure S51. HR-ESI-MS spectrum of compound 7 in $\mathrm{MeOH}$.

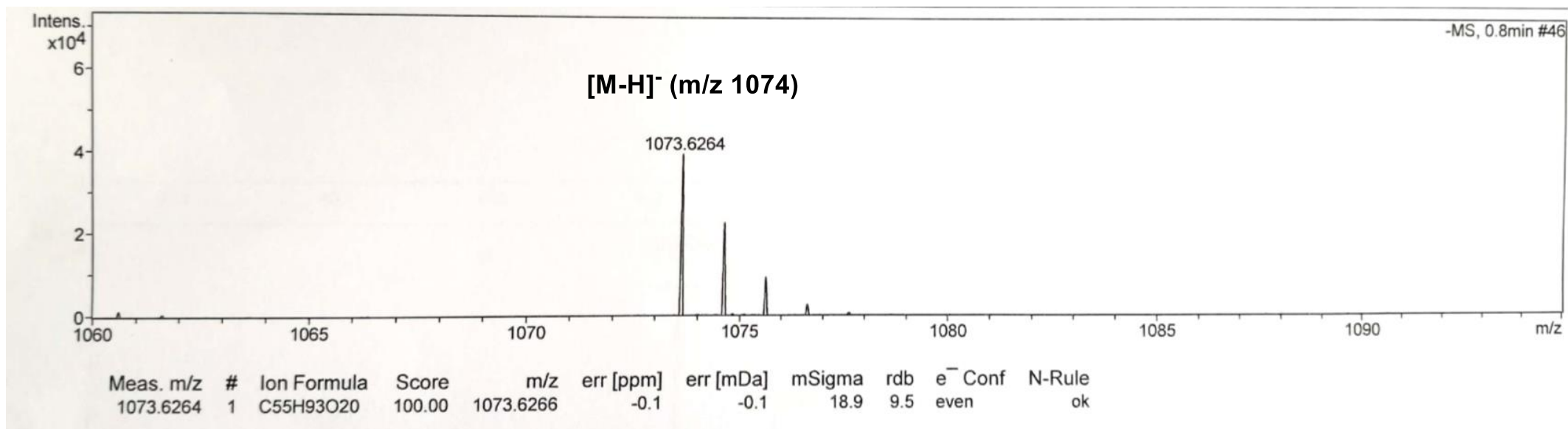

Figure S52. IR spectrum of compound 7.

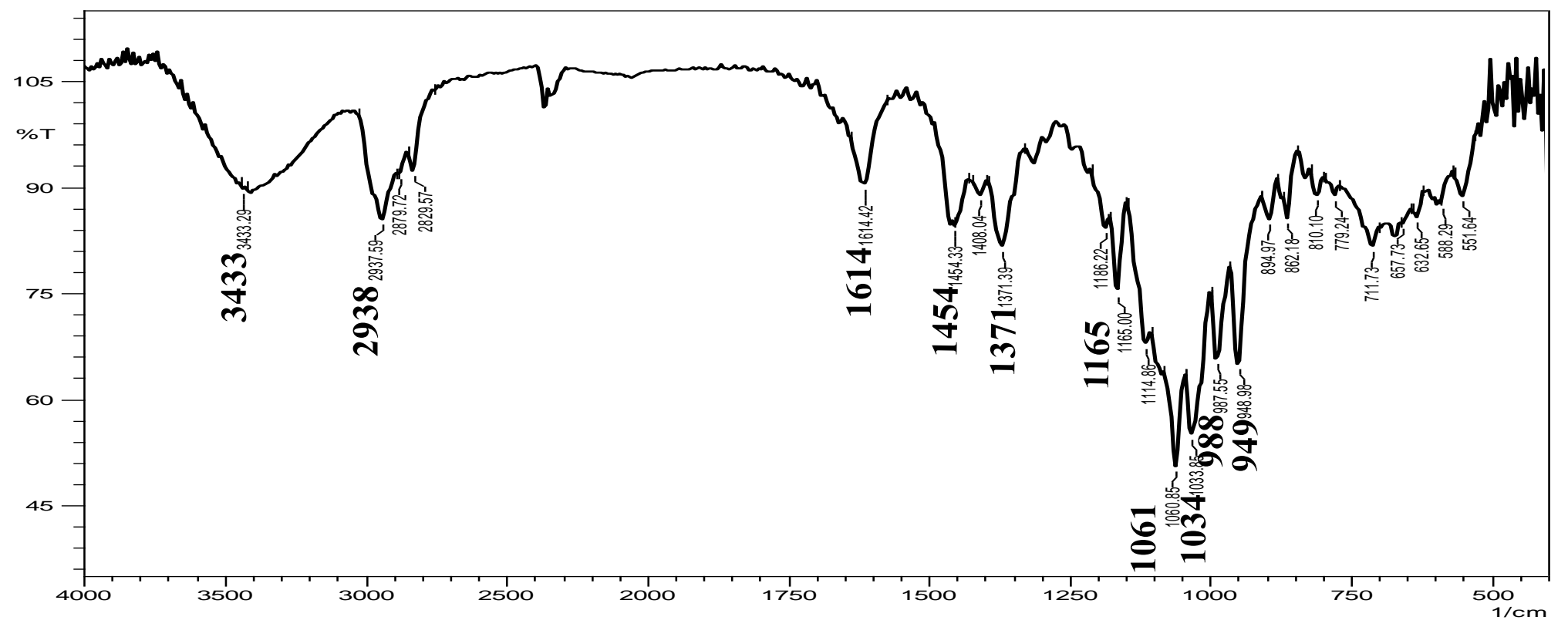


Figure S53. ${ }^{1} \mathrm{H}$ NMR $(700 \mathrm{MHz})$ spectrum of compound 7 in $\mathrm{CDCl}_{3}$.

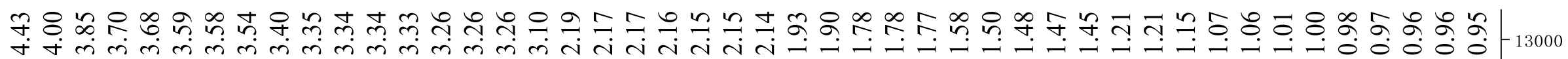

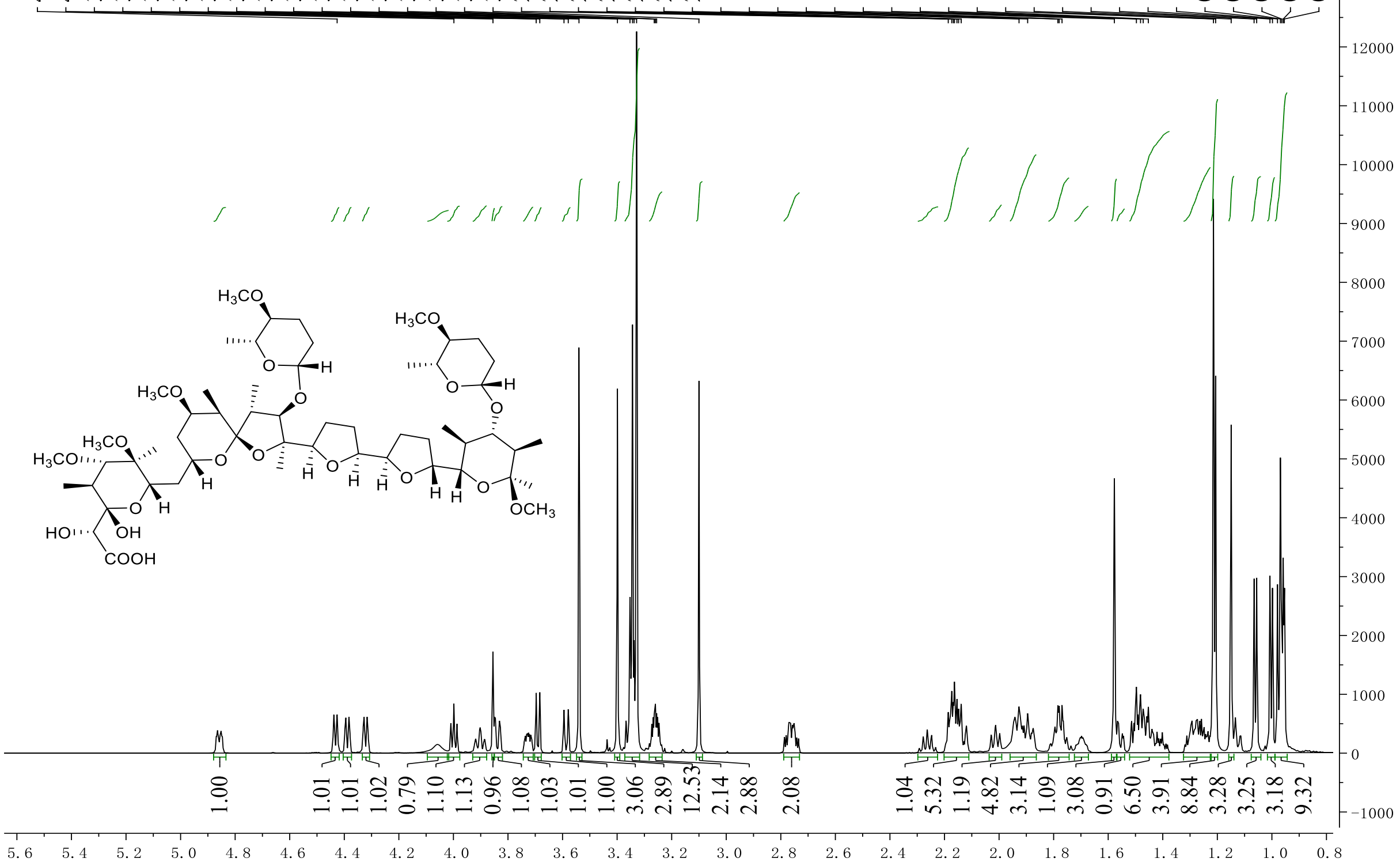


Figure S54. ${ }^{13} \mathrm{C}$ NMR $(175 \mathrm{MHz})$ spectrum of compound 7 in $\mathrm{CDCl}_{3}$.

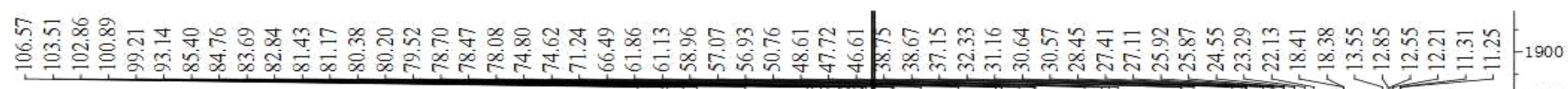
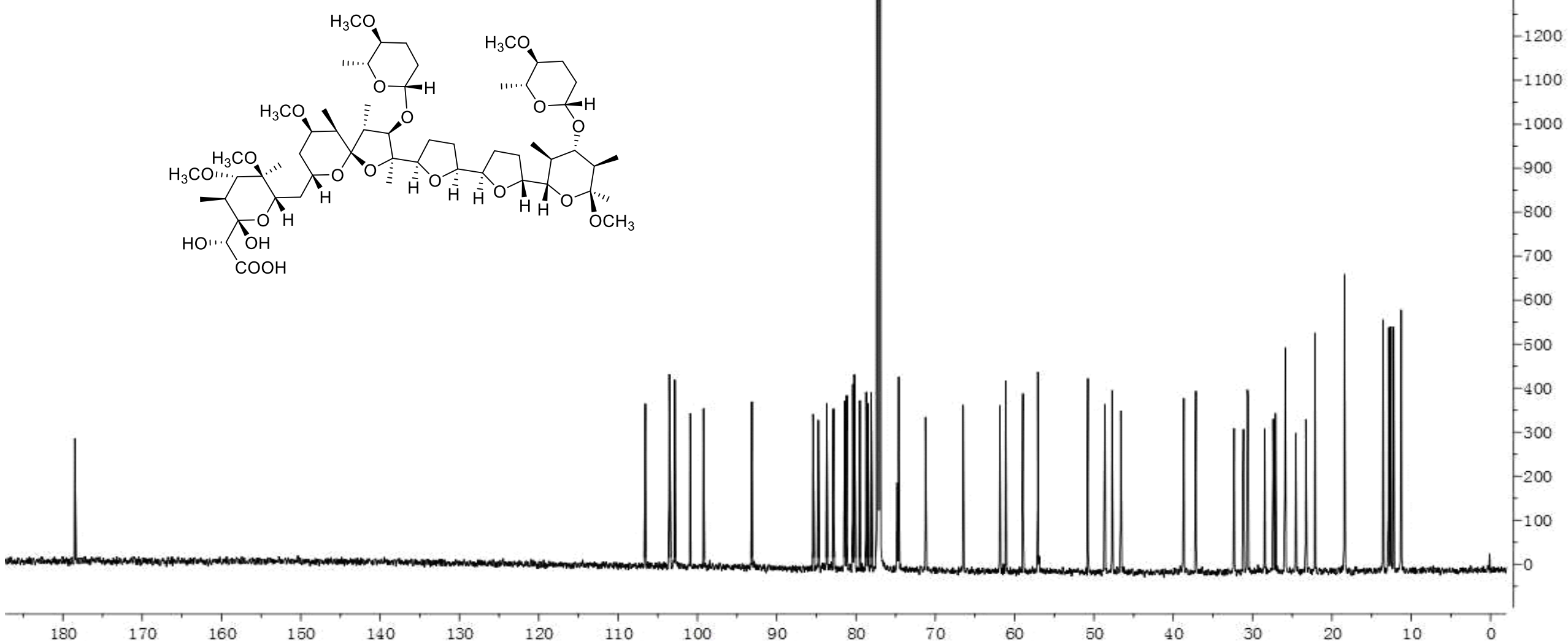
Figure S55. ${ }^{13} \mathrm{C}$ DEPT spectrum of compound 7 in $\mathrm{CDCl}_{3}$.

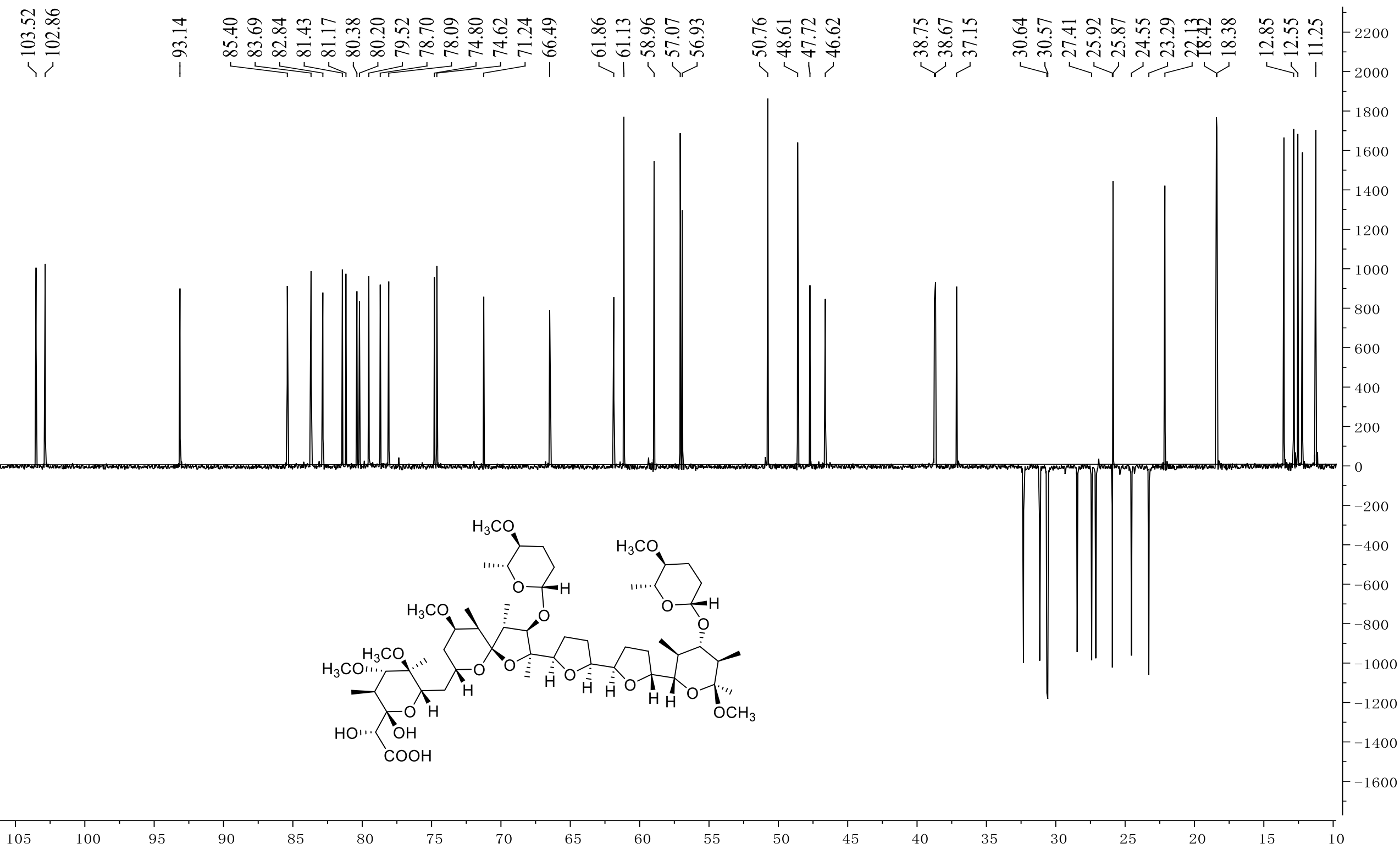


Figure S56. ${ }^{1} \mathrm{H}-{ }^{1} \mathrm{H}$ COSY spectrum of compound 7 in $\mathrm{CDCl}_{3}$.

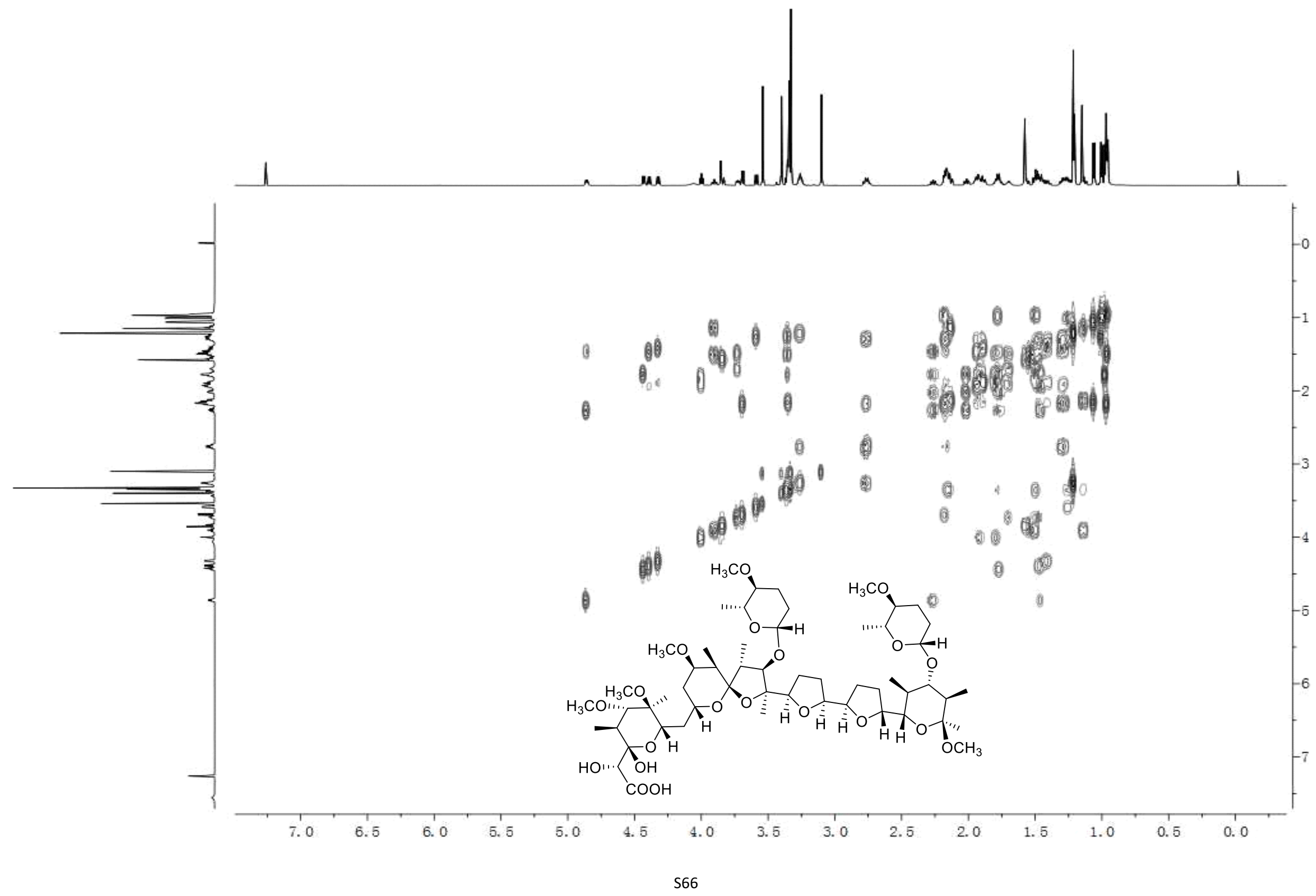


Figure S57. HSQC spectrum of compound 7 in $\mathrm{CDCl}_{3}$.

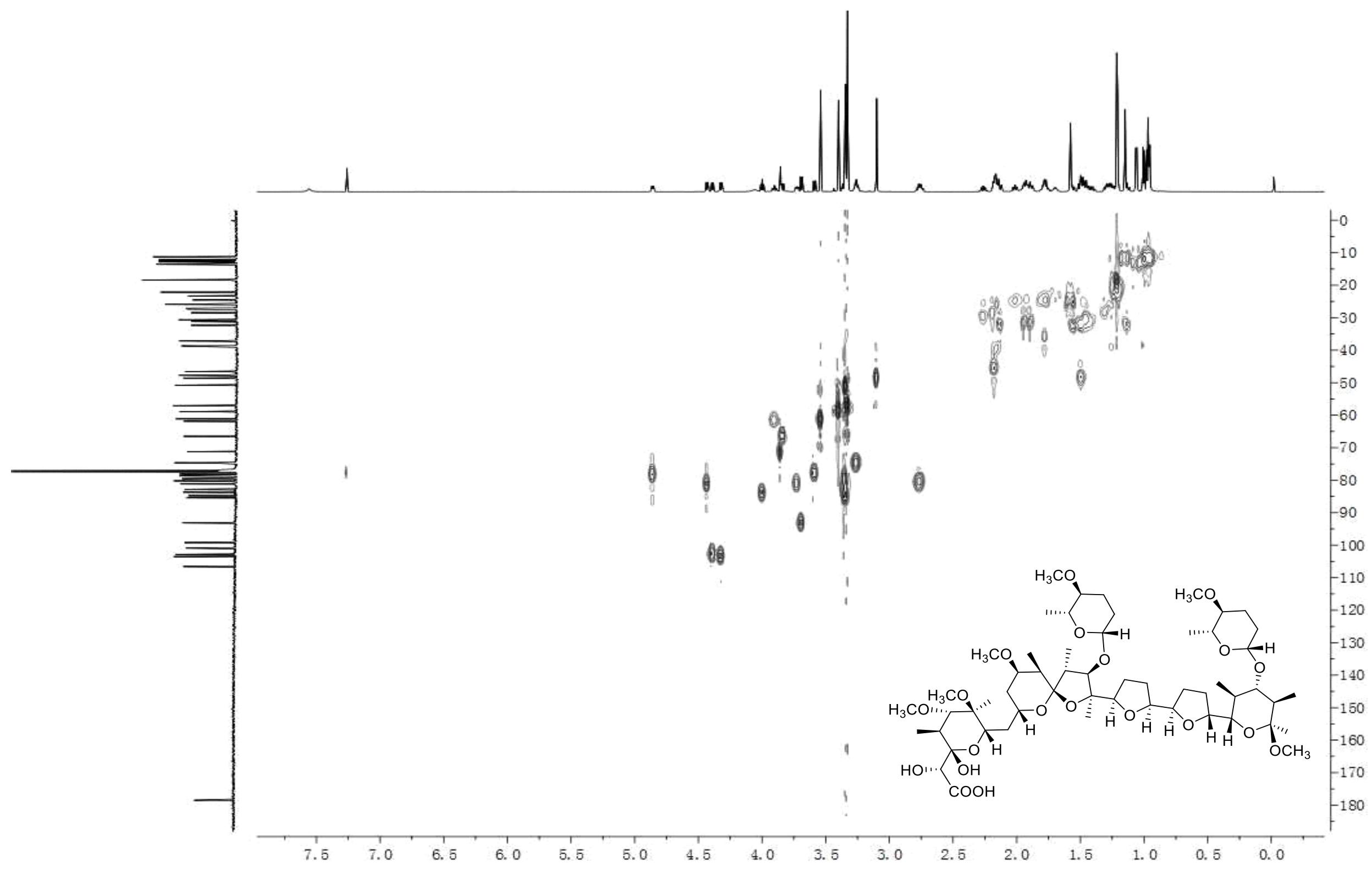


Figure S58. HMBC spectrum of compound 7 in $\mathrm{CDCl}_{3}$.

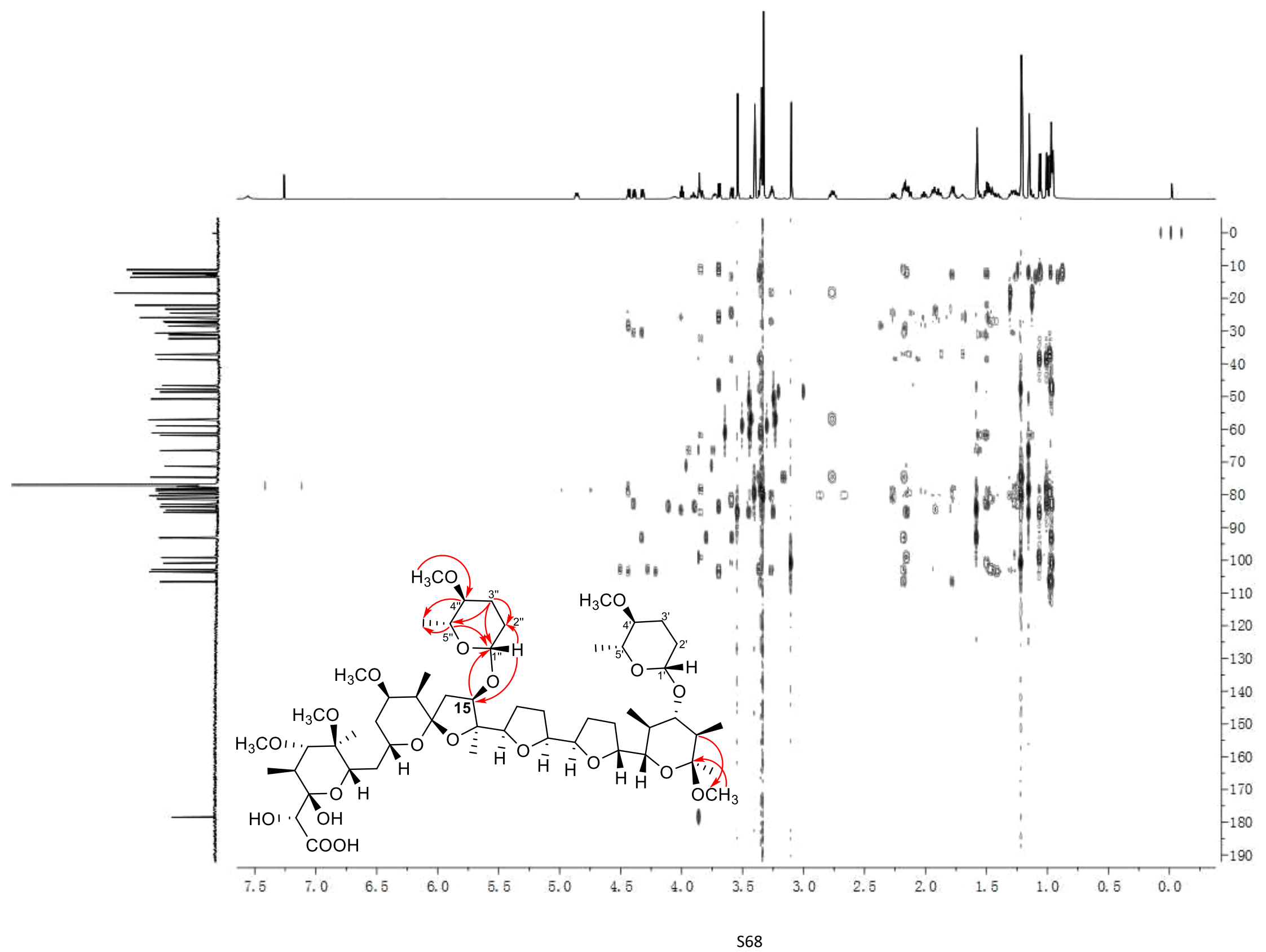


Figure S59. NOESY spectrum of compound 7 in $\mathrm{CDCl}_{3}$.

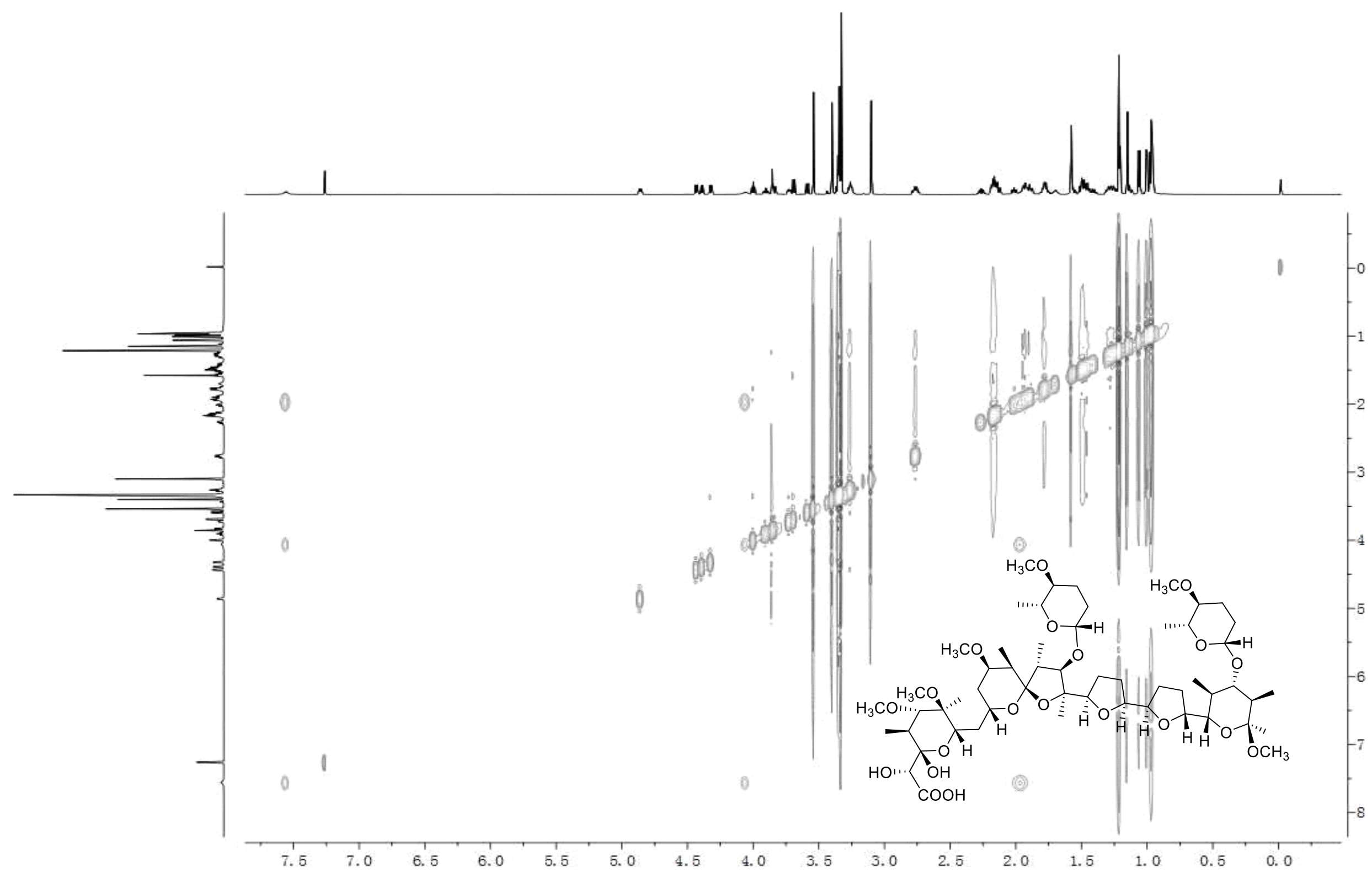


Figure S60. HR-ESI-MS spectrum of compound 8 in $\mathrm{MeOH}$.

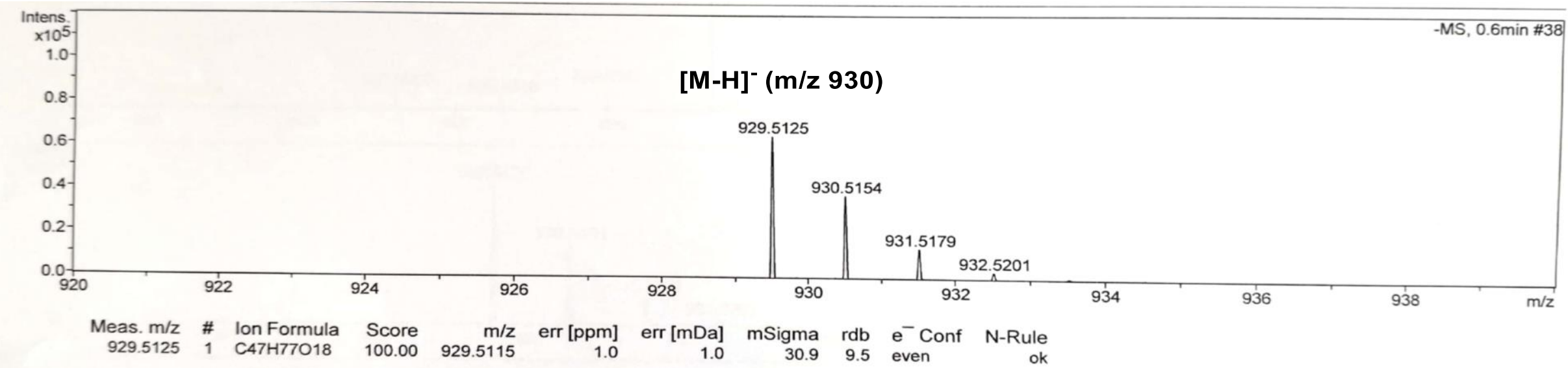

Figure S61. IR spectrum of compound $\mathbf{8}$.

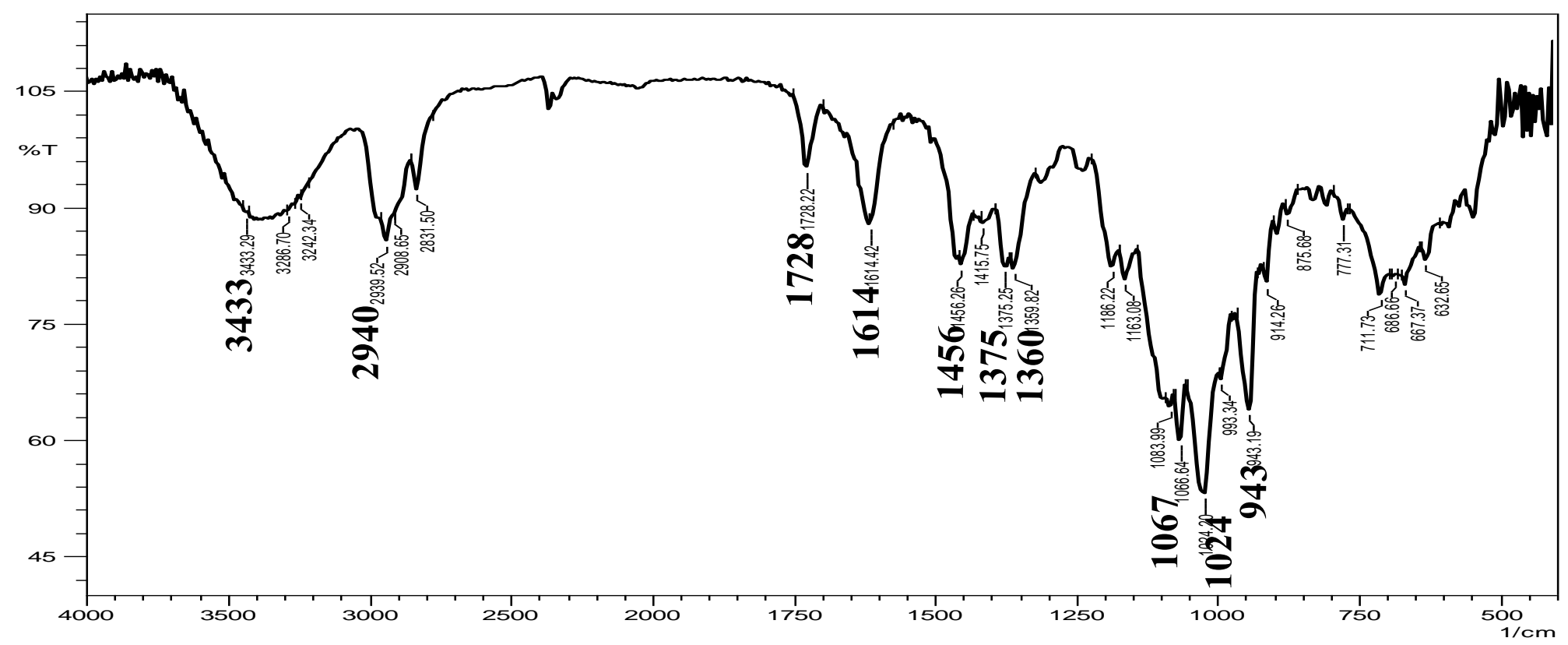


Figure S62. ${ }^{1} \mathrm{H}$ NMR $(700 \mathrm{MHz})$ spectrum of compound 8 in $\mathrm{CDCl}_{3}$.

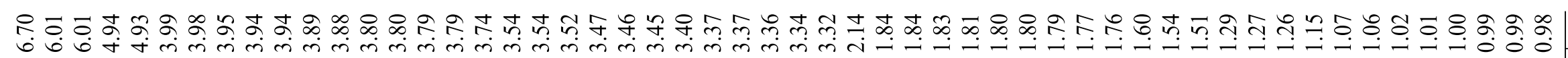

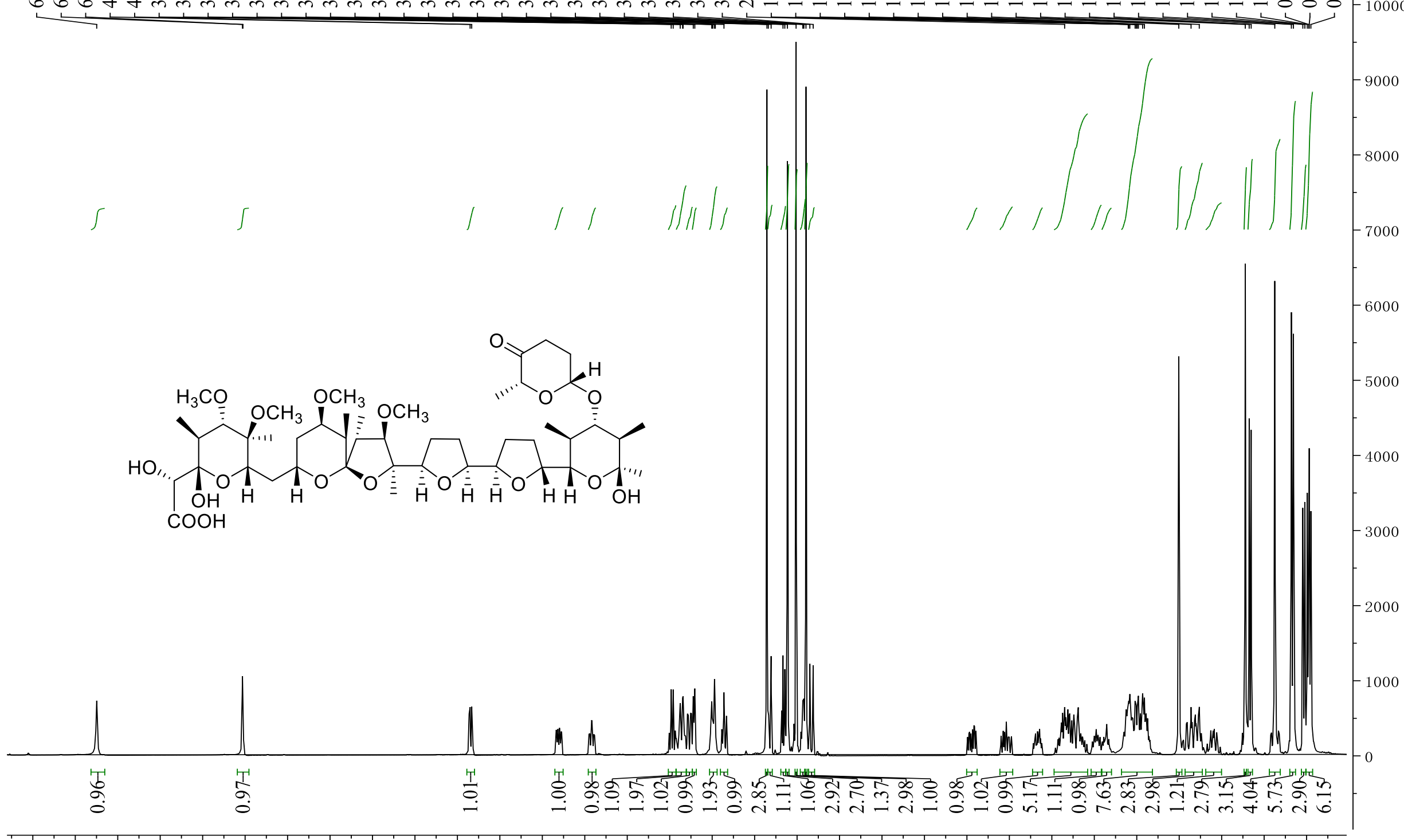

$\begin{array}{llllllllllllllllllllllllllllllll}7.0 & 6.8 & 6.6 & 6.4 & 6.2 & 6.0 & 5.8 & 5.6 & 5.4 & 5.2 & 5.0 & 4.8 & 4.6 & 4.4 & 4.2 & 4.0 & 3.8 & 3.6 & 3.4 & 3.2 & 3.0 & 2.8 & 2.6 & 2.4 & 2.2 & 2.0 & 1.8 & 1.6 & 1.4 & 1.2 & 1.0\end{array}$ 
Figure S63. ${ }^{13} \mathrm{C}$ NMR $(175 \mathrm{MHz})$ spectrum of compound 8 in $\mathrm{CDCl}_{3}$.

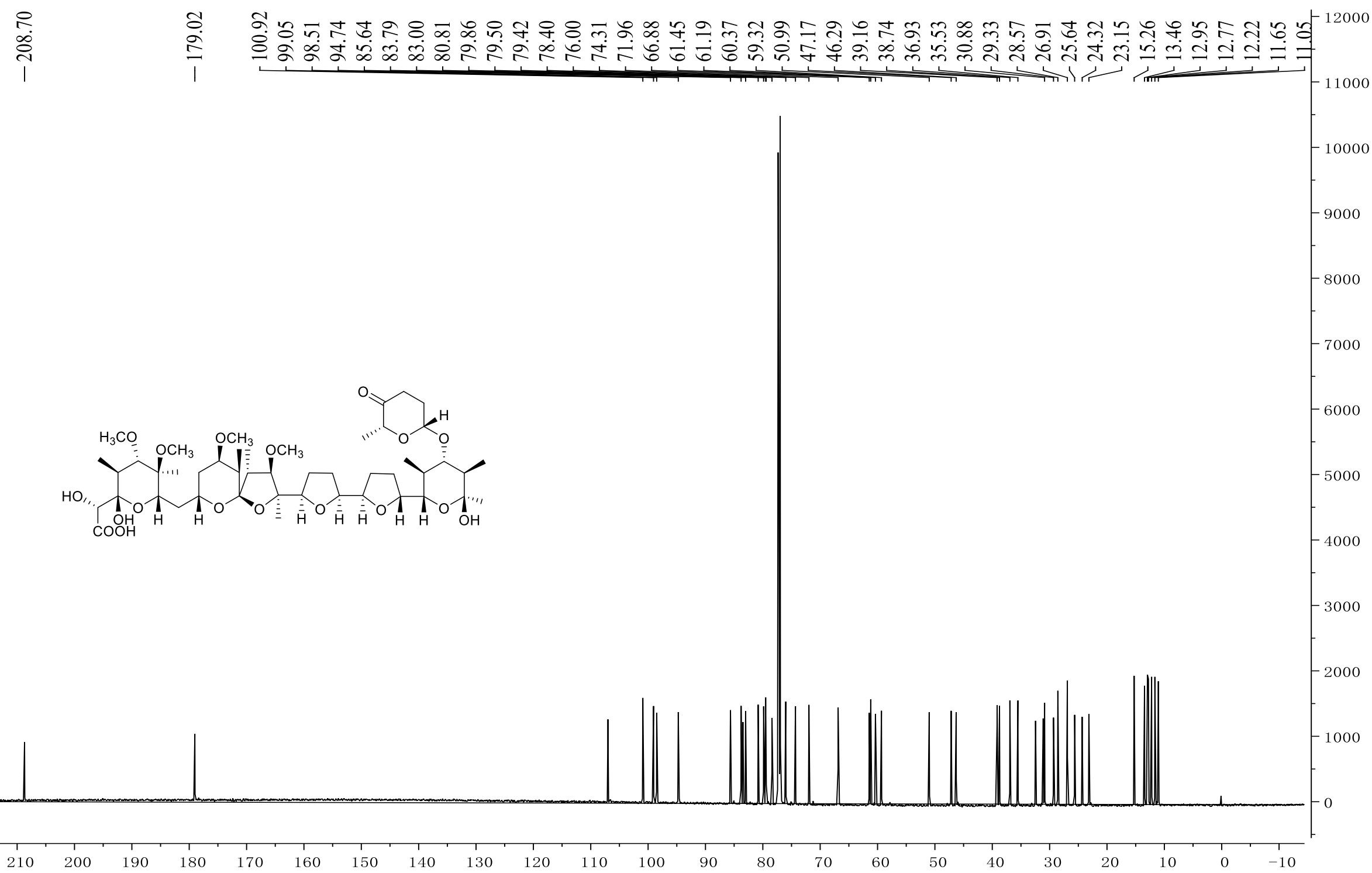


Figure S64. ${ }^{13} \mathrm{C}$ DEPT spectrum of compound 8 in $\mathrm{CDCl}_{3}$.

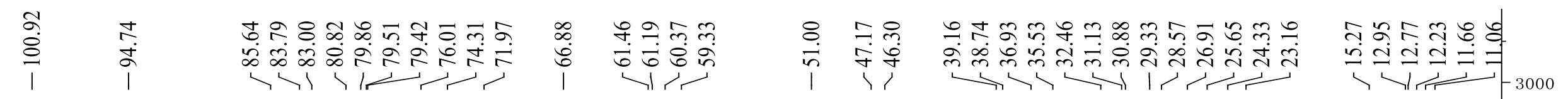

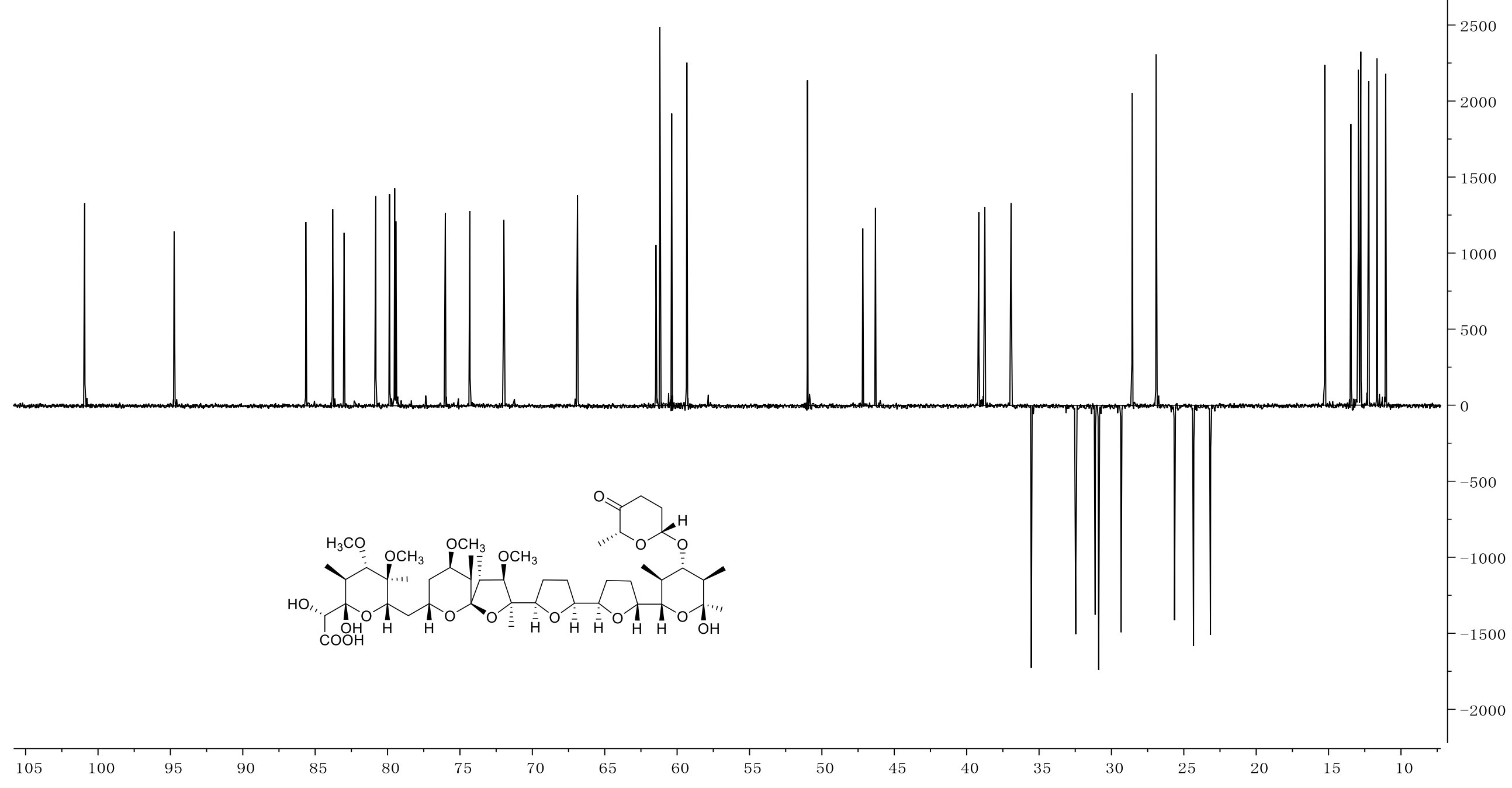


Figure S65. ${ }^{1} \mathrm{H}-{ }^{1} \mathrm{H}$ COSY spectrum of compound 8 in $\mathrm{CDCl}_{3}$.

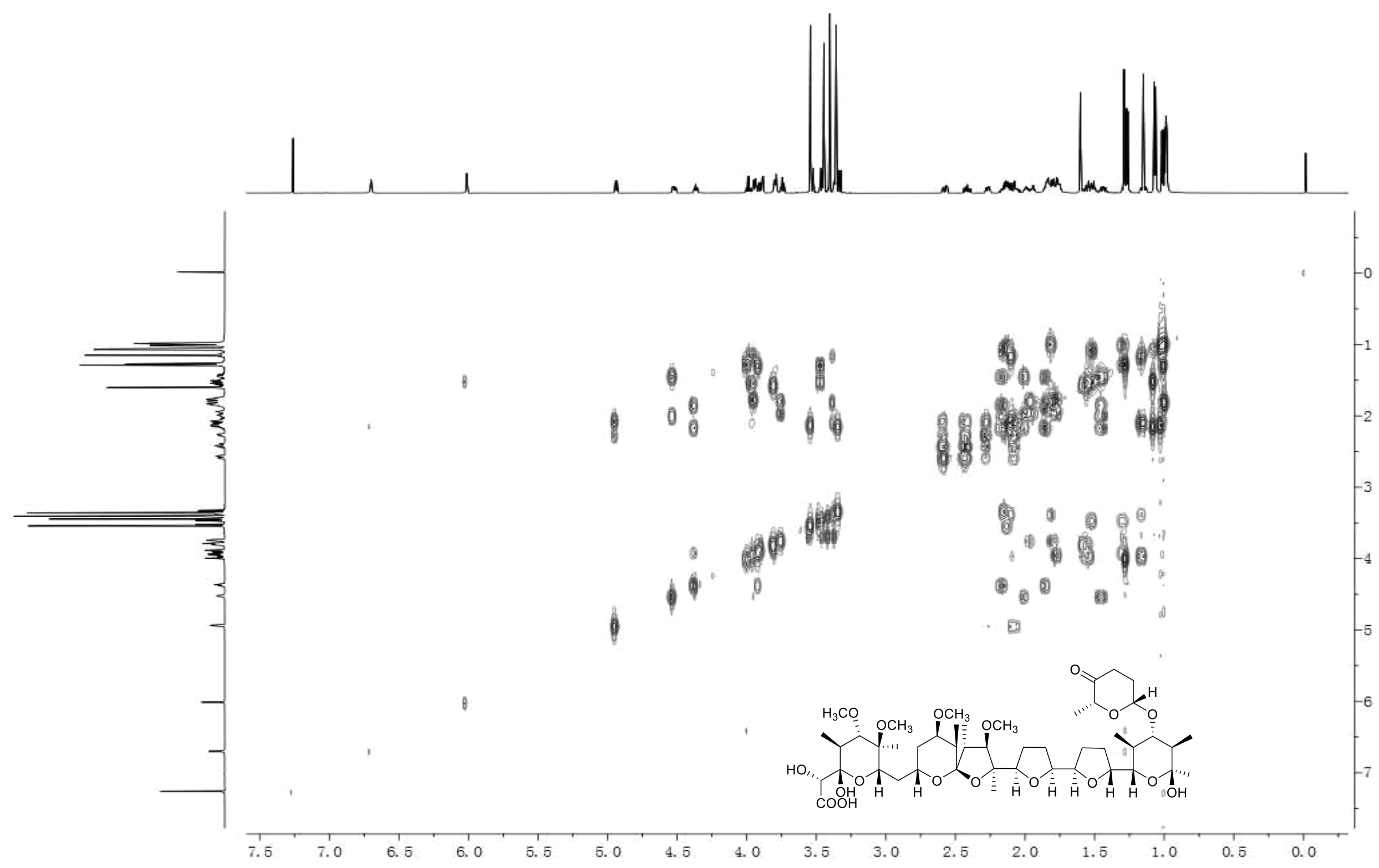


Figure S66. HSQC spectrum of compound $\mathbf{8}$ in $\mathrm{CDCl}_{3}$.

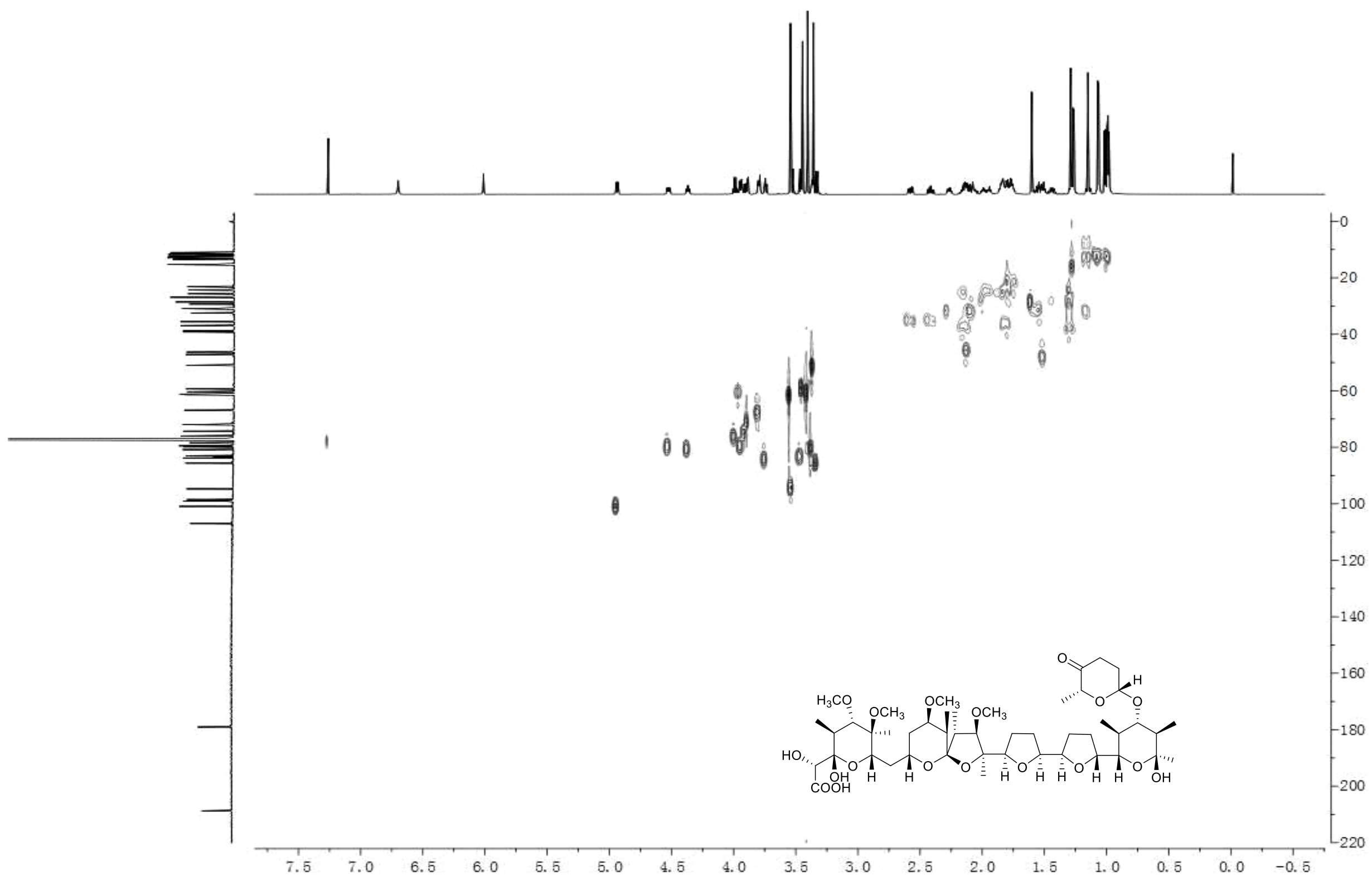


Figure S67. HMBC spectrum of compound $\mathbf{8}$ in $\mathrm{CDCl}_{3}$.

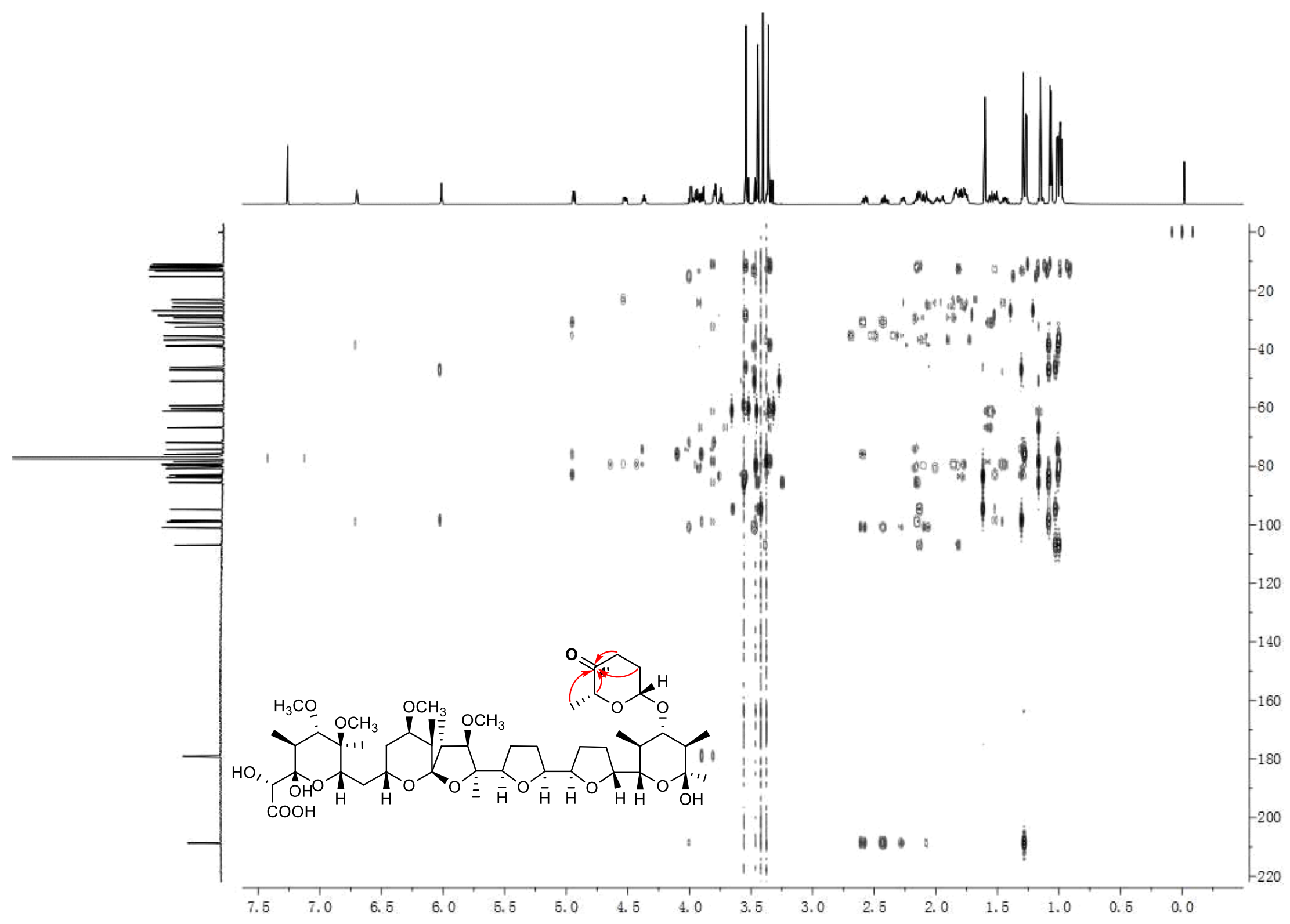


Figure S68. NOESY spectrum of compound 8 in $\mathrm{CDCl}_{3}$.

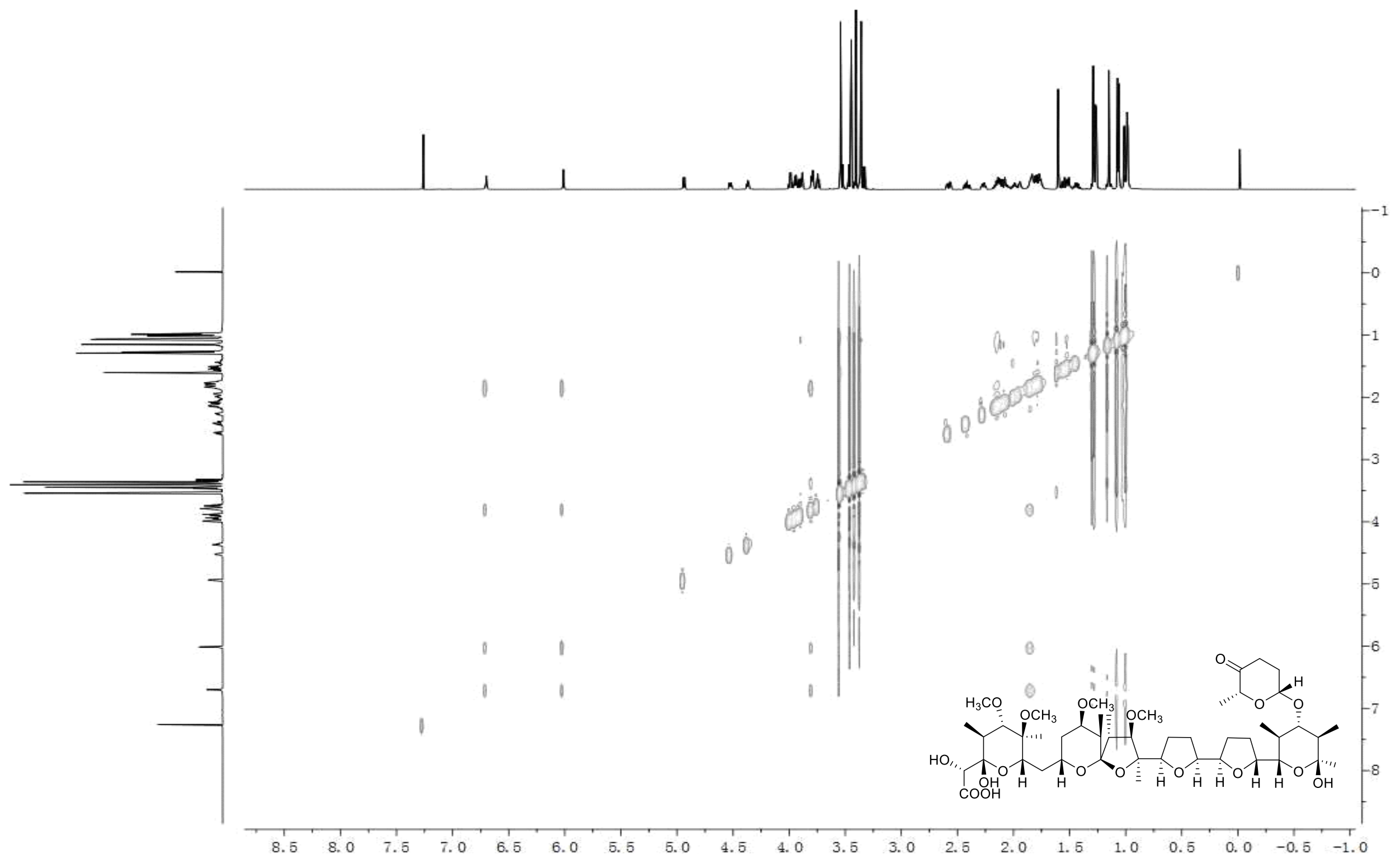


Figure S69. HR-ESI-MS spectrum of compound 9 in $\mathrm{MeOH}$.

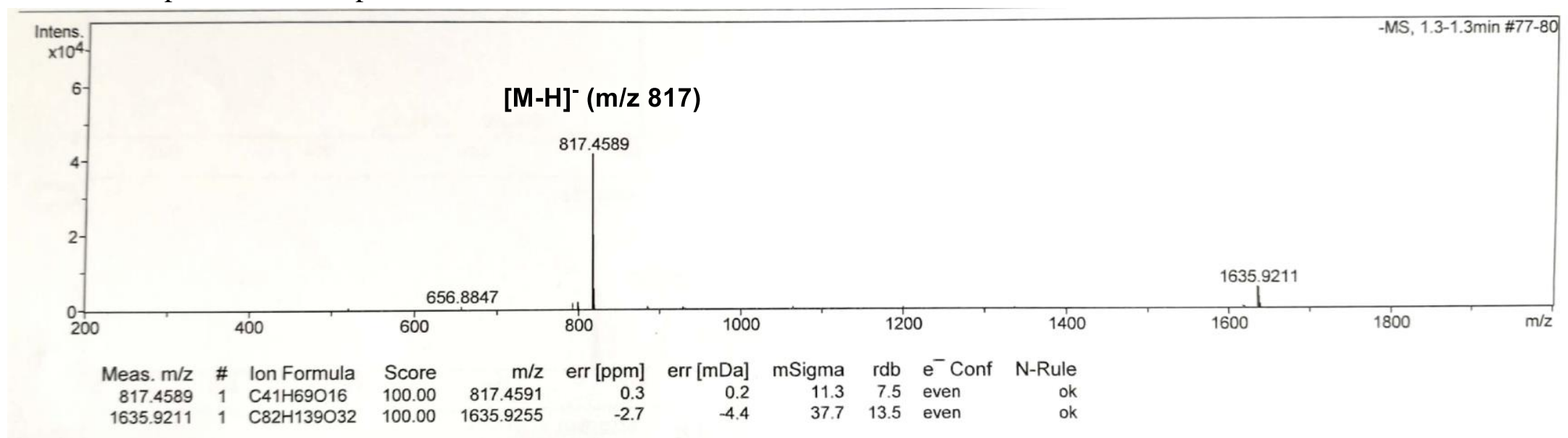

Figure S70. IR spectrum of compound 9.

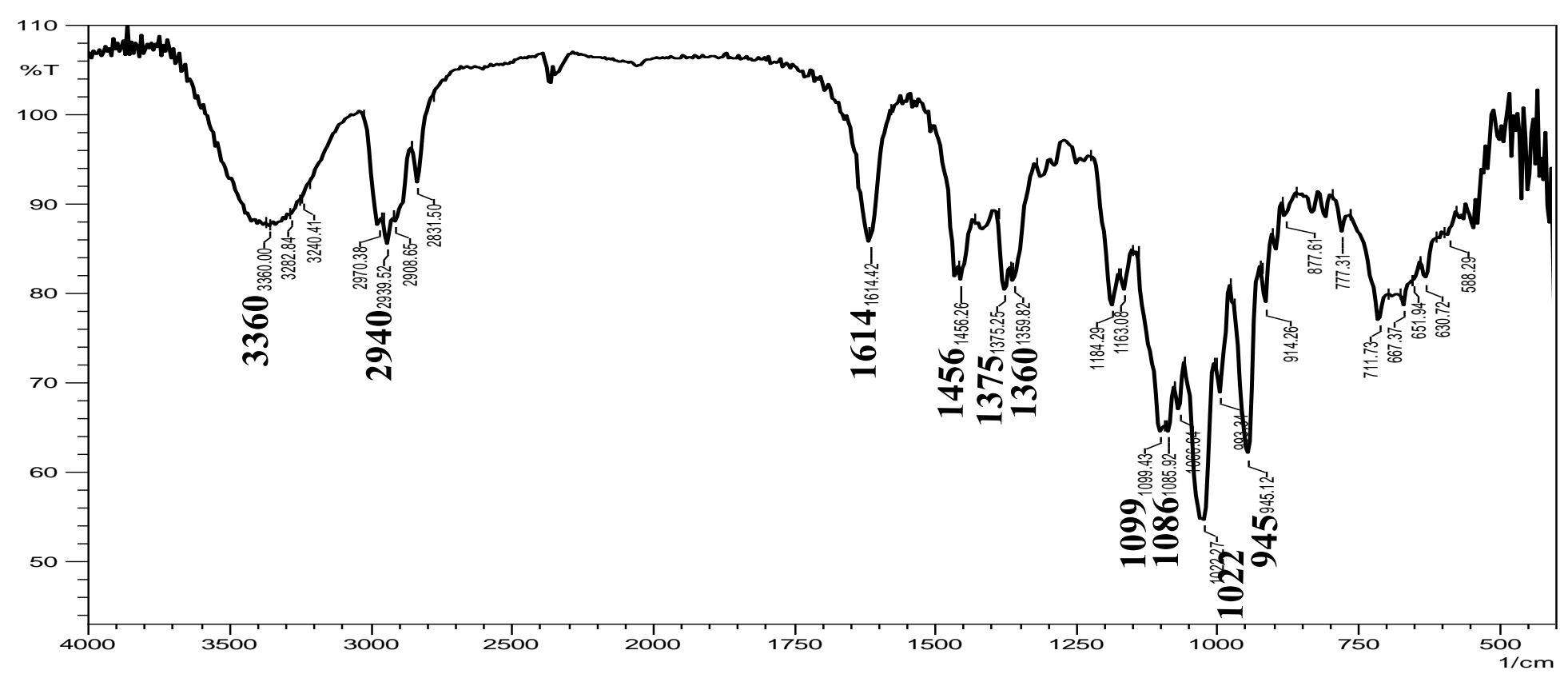


Figure S71. ${ }^{1} \mathrm{H}$ NMR $(700 \mathrm{MHz})$ spectrum of compound 9 in $\mathrm{CDCl}_{3}$.

宓

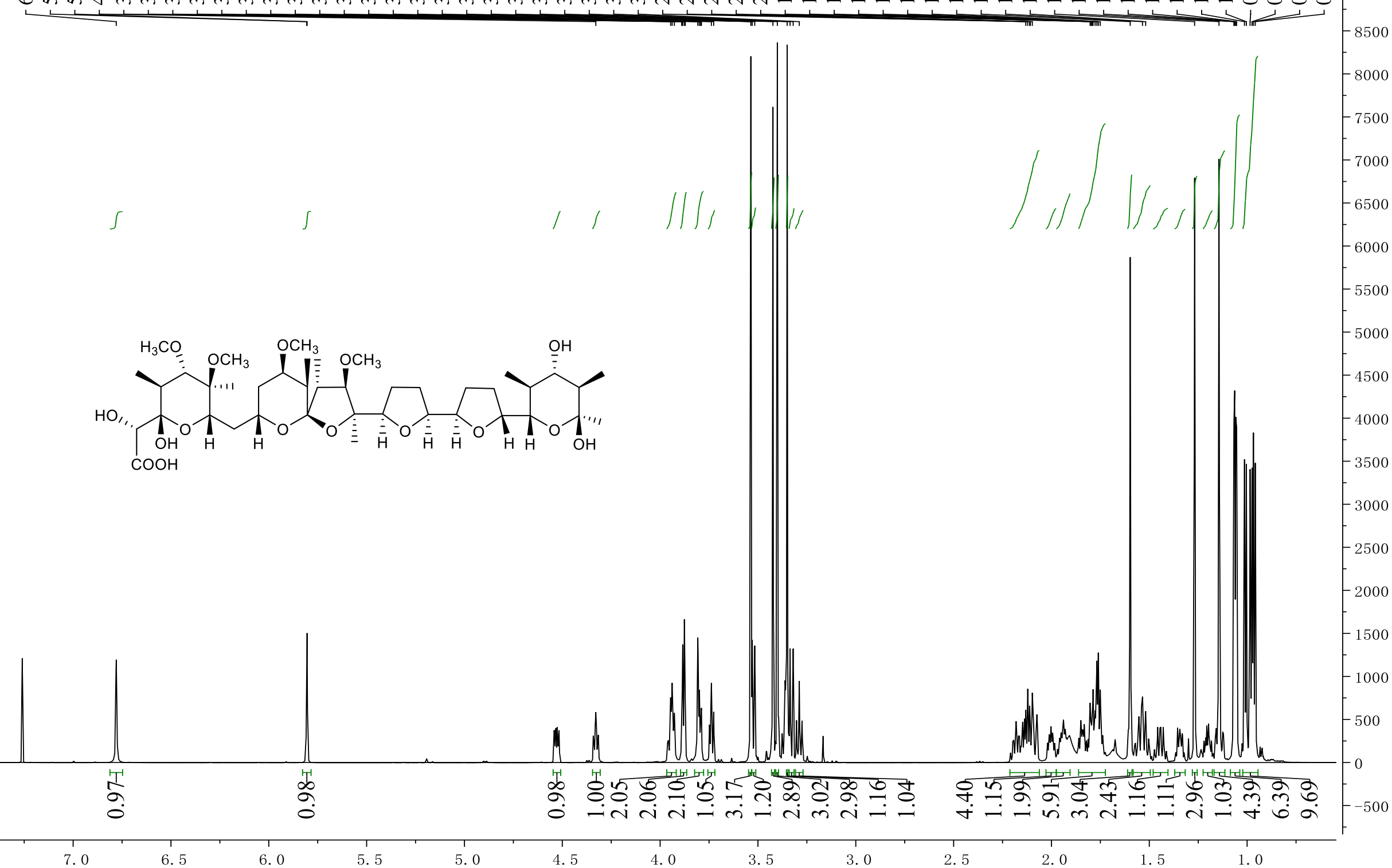


Figure S72. ${ }^{13} \mathrm{C}$ NMR $(175 \mathrm{MHz})$ spectrum of compound 9 in $\mathrm{CDCl}_{3}$.

$\stackrel{\substack{\infty \\ ٍ}}{\stackrel{1}{\leftrightarrows}}$

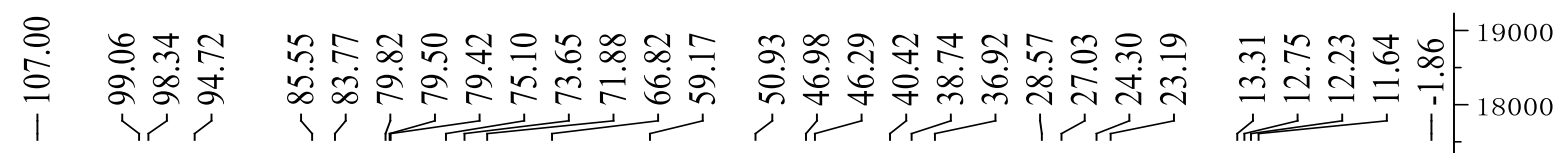

$\mathrm{H}_{3} \mathrm{CO}$
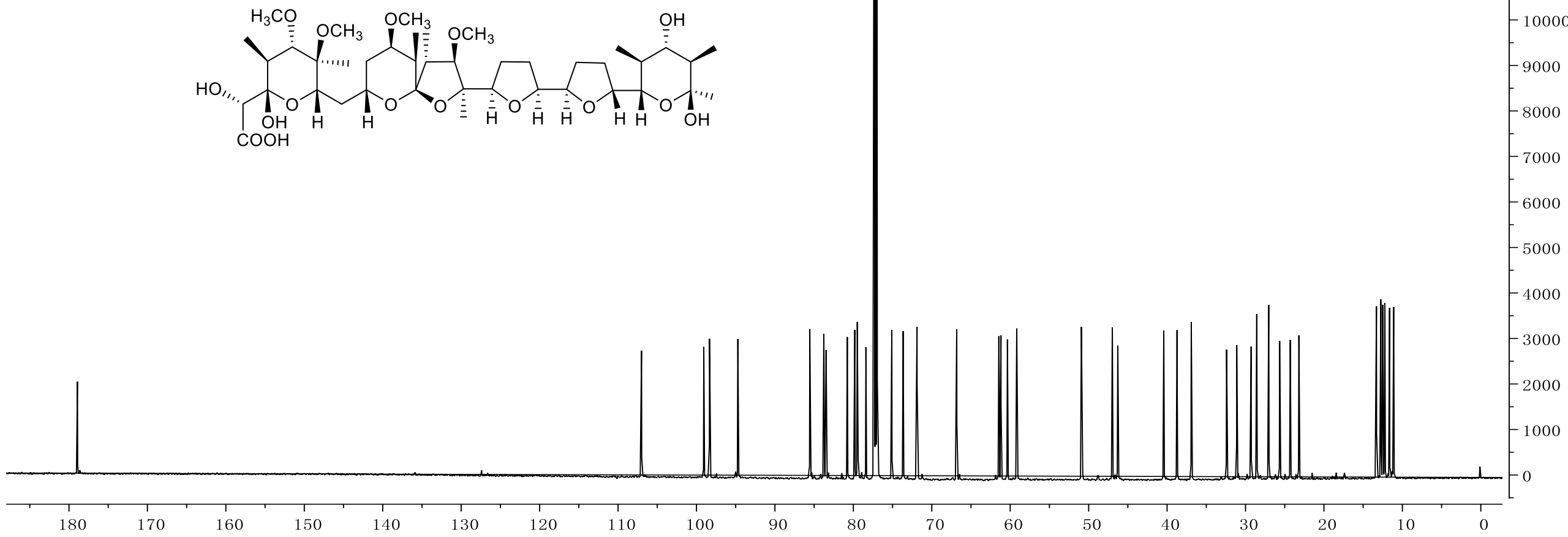
Figure S73. ${ }^{13} \mathrm{C}$ DEPT spectrum of compound 9 in $\mathrm{CDCl}_{3}$.

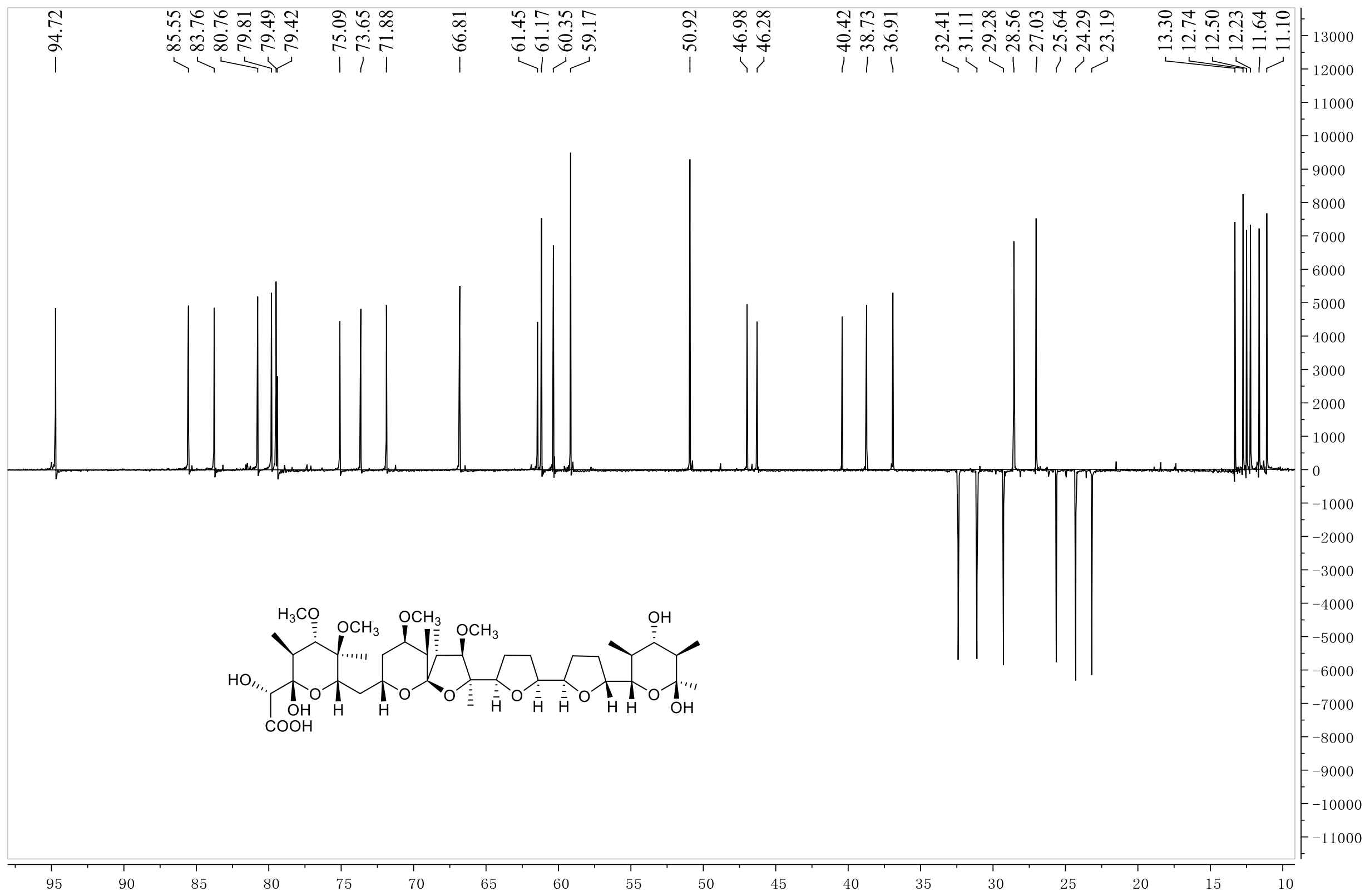


Figure S74. ${ }^{1} \mathrm{H}-{ }^{1} \mathrm{H}$ COSY spectrum of compound 9 in $\mathrm{CDCl}_{3}$.

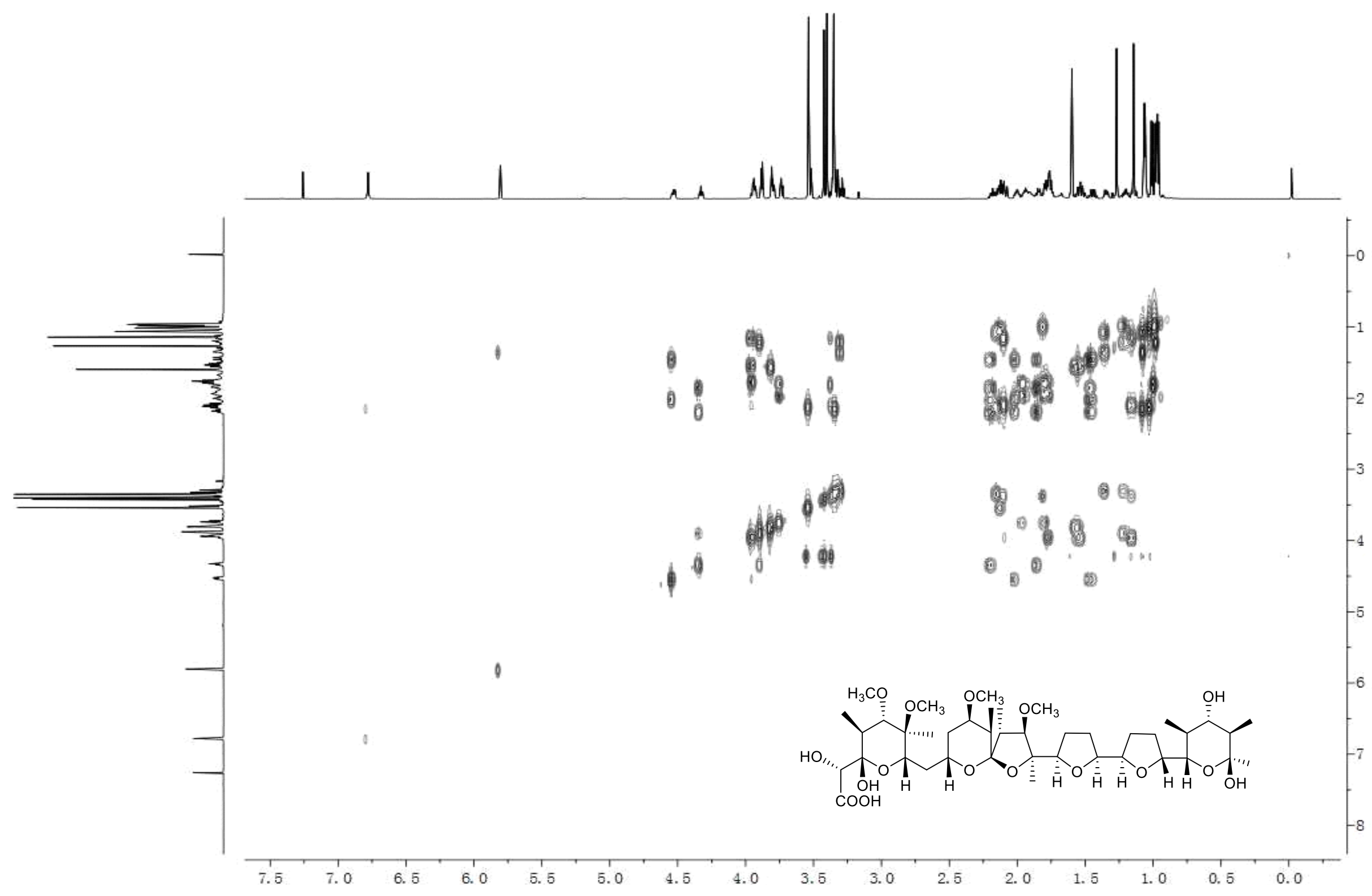


Figure S75. HSQC spectrum of compound 9 in $\mathrm{CDCl}_{3}$.

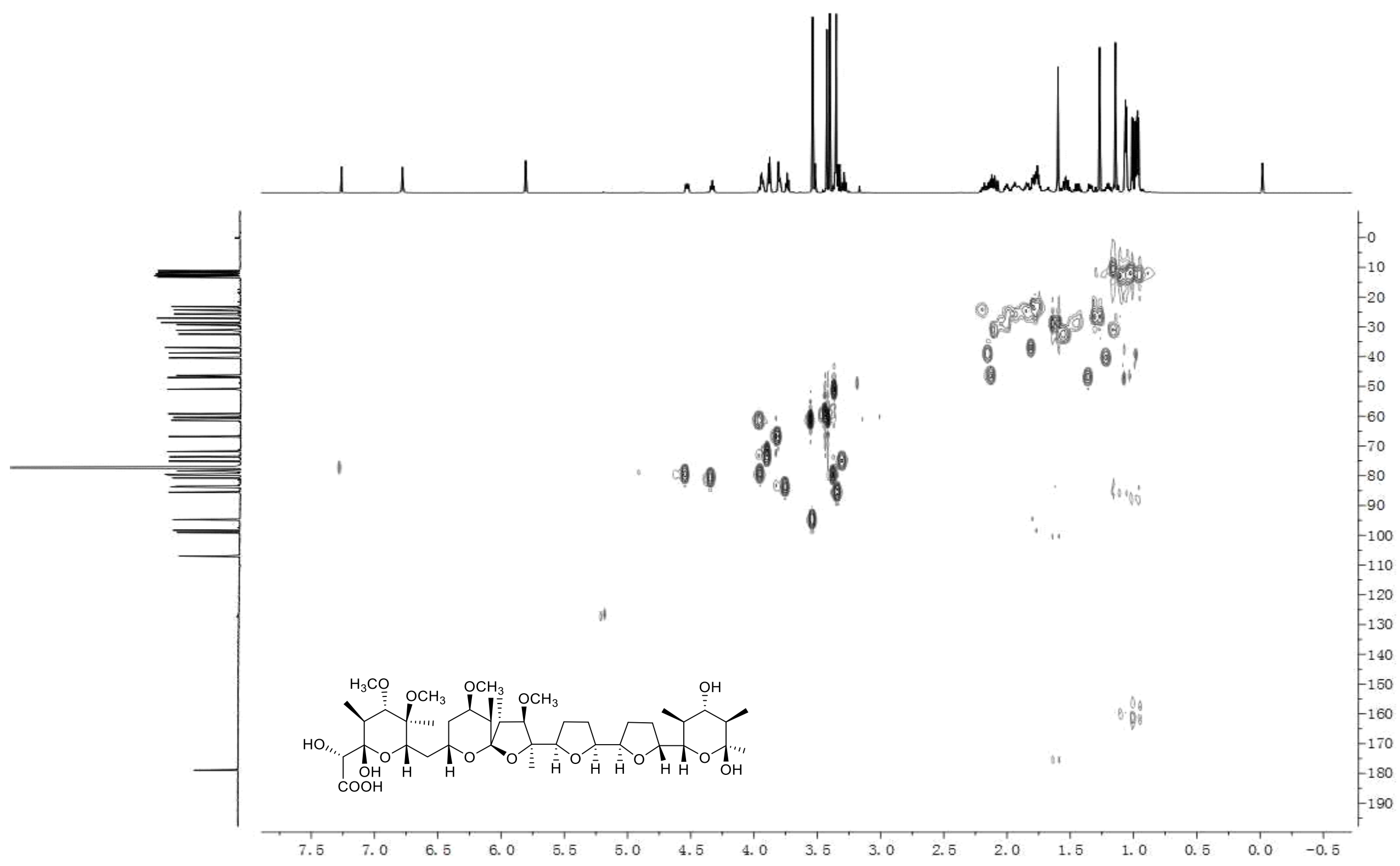


Figure S76. $\mathrm{HMBC}$ spectrum of compound 9 in $\mathrm{CDCl}_{3}$.

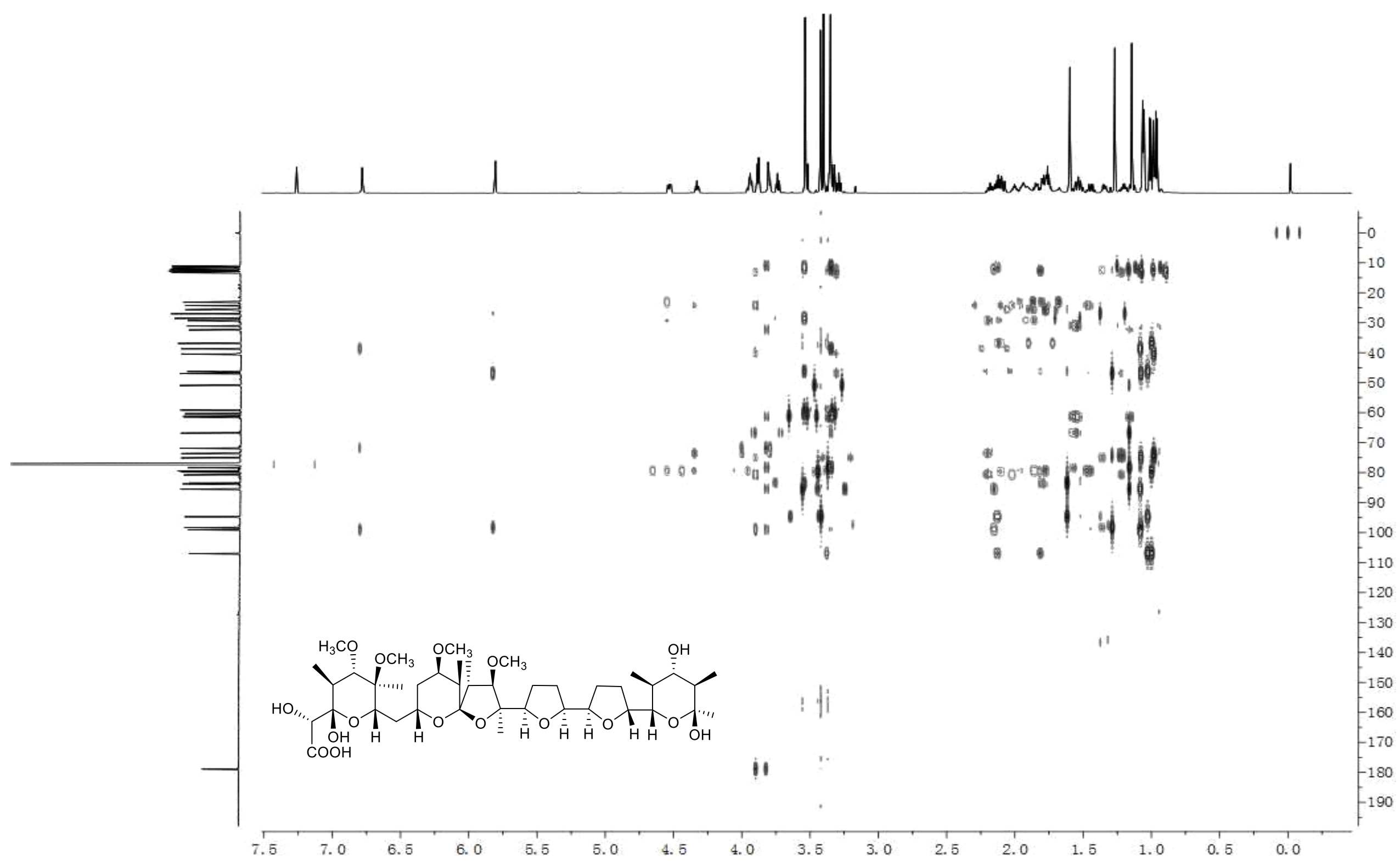


Figure S77. NOESY spectrum of compound 9 in $\mathrm{CDCl}_{3}$.

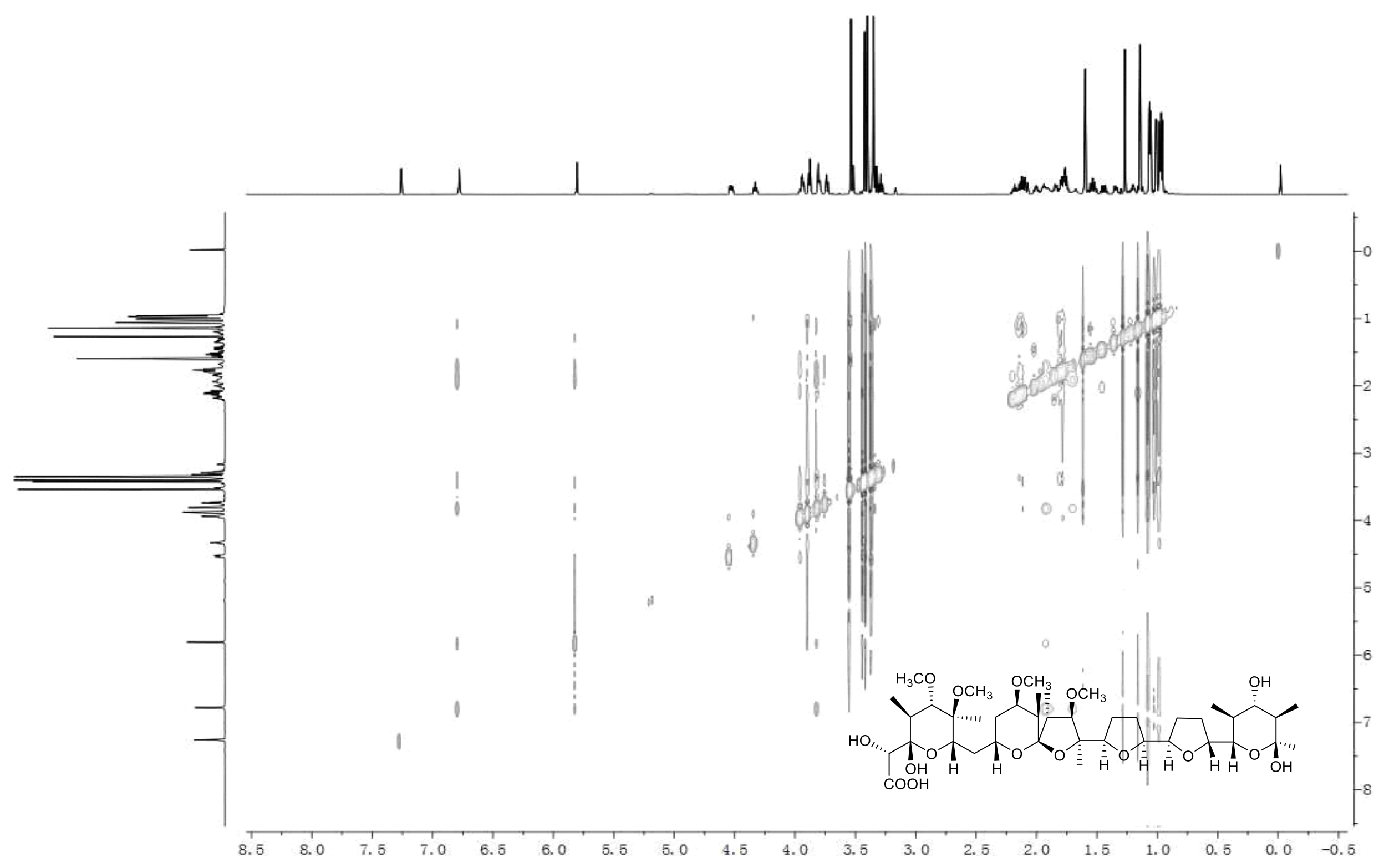


Figure S78. HR-ESI-MS spectrum of compound 10 in $\mathrm{MeOH}$.

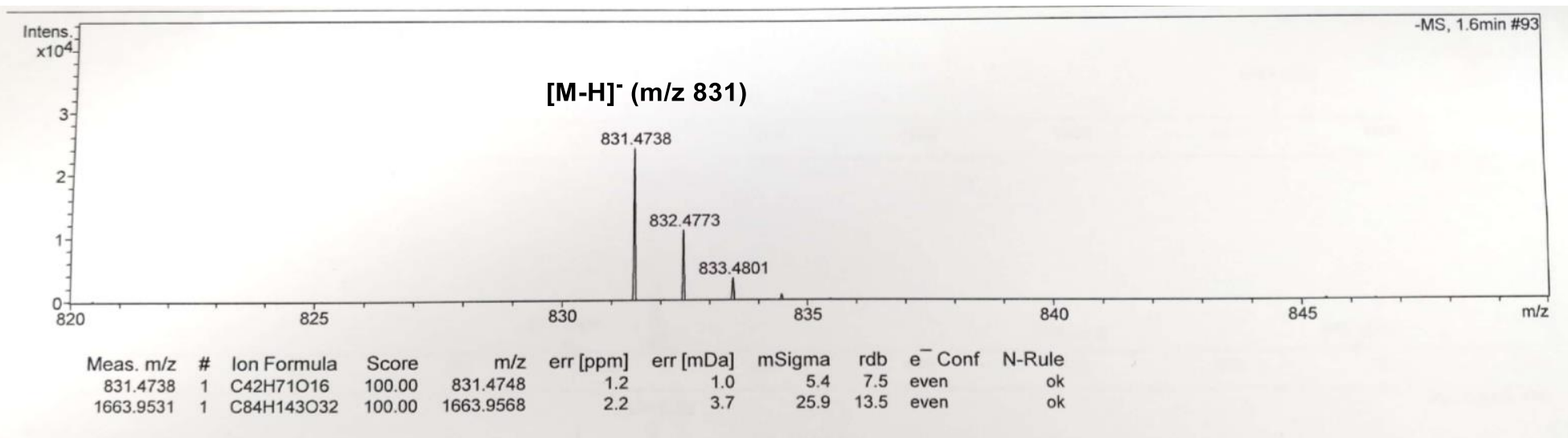

Figure S79. IR spectrum of compound $\mathbf{1 0 .}$

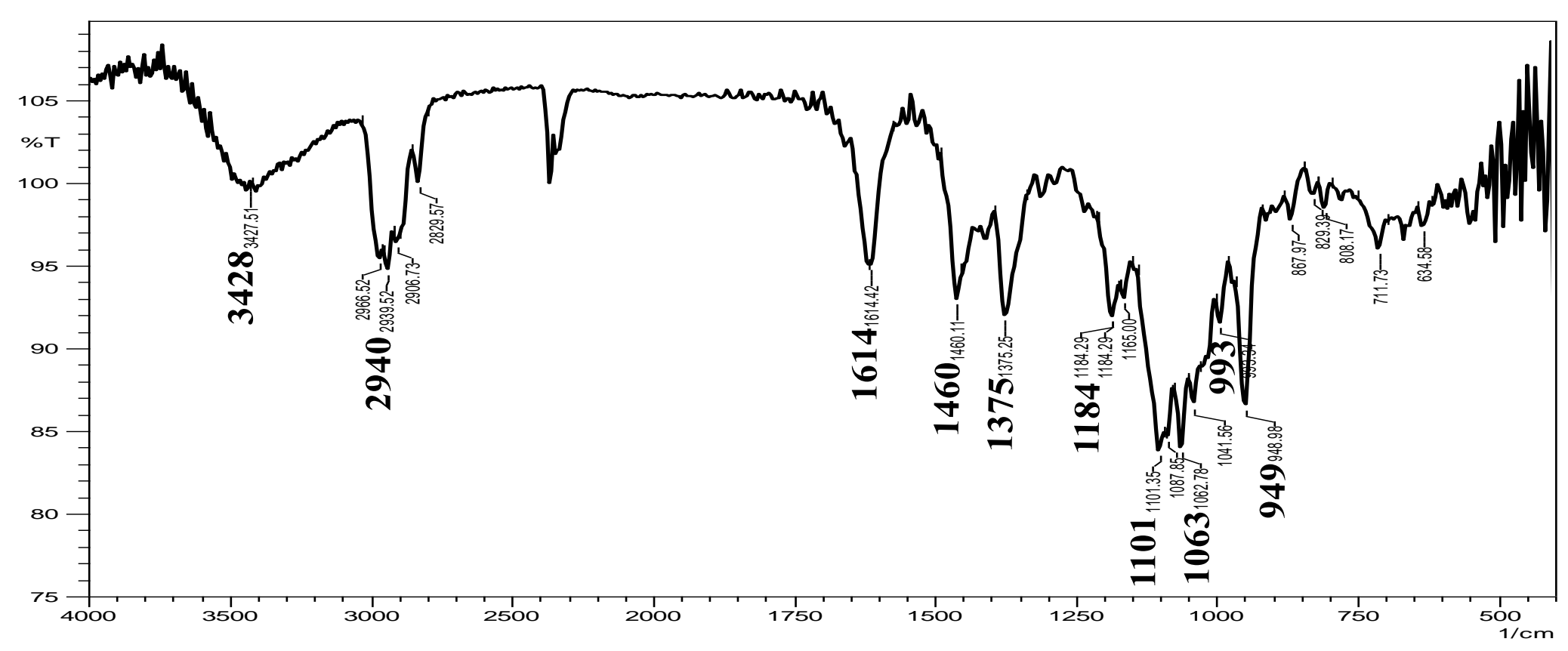


Figure S80. ${ }^{1} \mathrm{H}$ NMR $(700 \mathrm{MHz})$ spectrum of compound 10 in $\mathrm{CDCl}_{3}$.

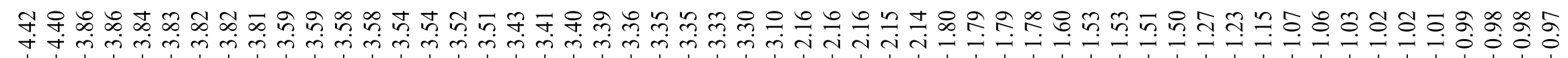

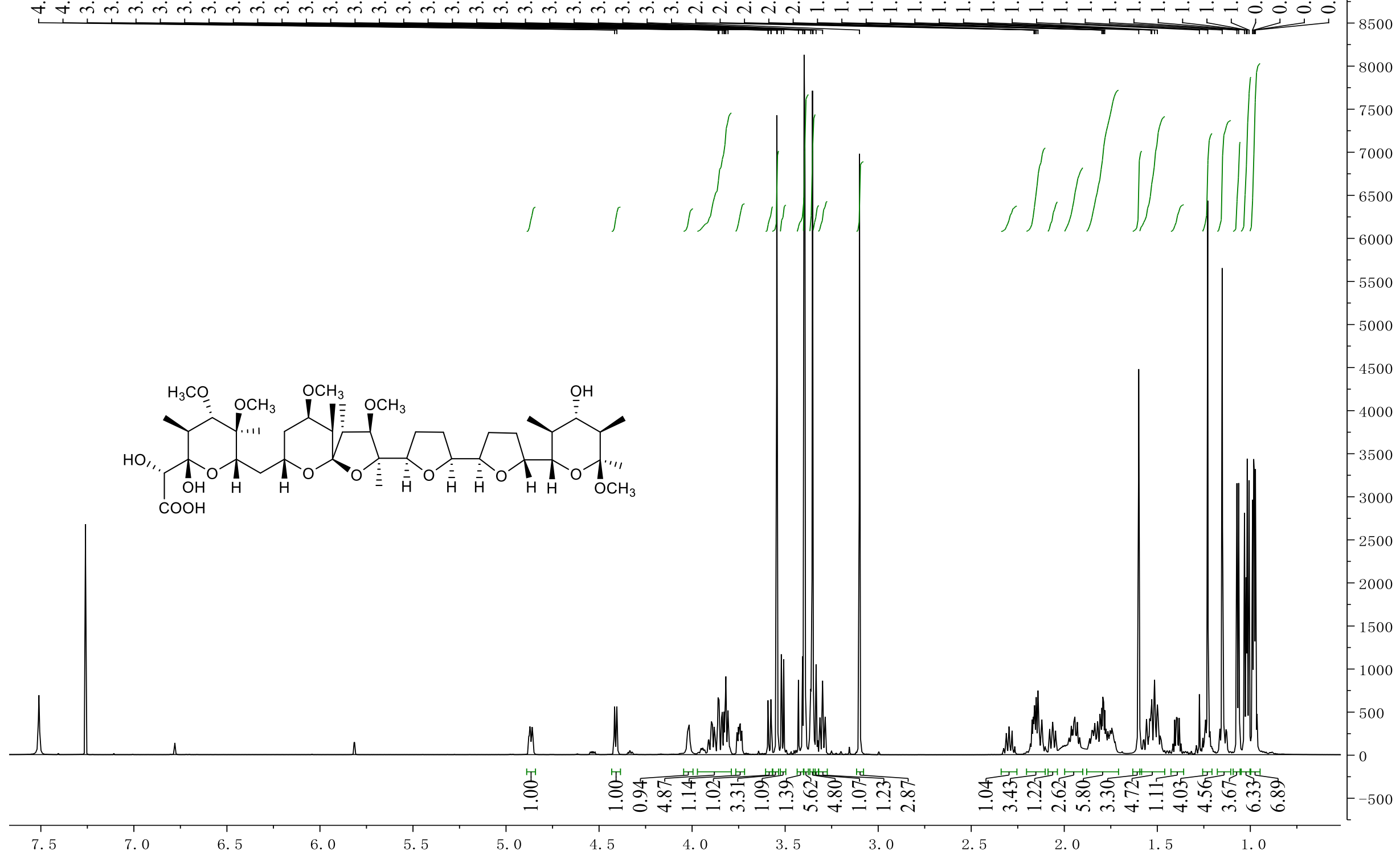


Figure S81. ${ }^{13} \mathrm{C}$ NMR $(175 \mathrm{MHz})$ spectrum of compound $\mathbf{1 0}$ in $\mathrm{CDCl}_{3}$.

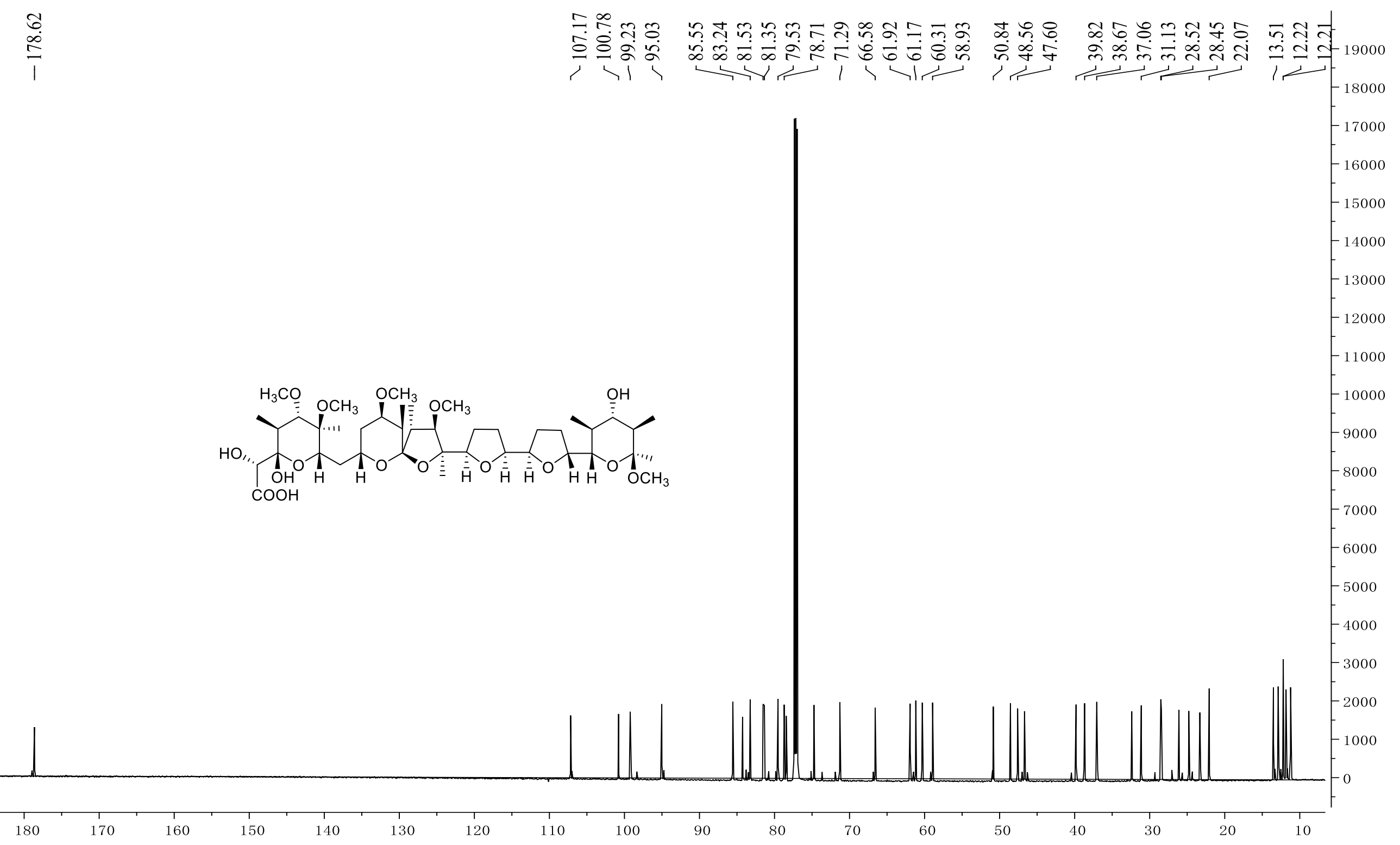


Figure S82. ${ }^{13} \mathrm{C}$ DEPT spectrum of compound 10 in $\mathrm{CDCl}_{3}$.

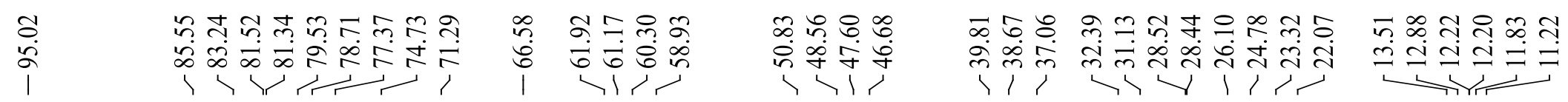

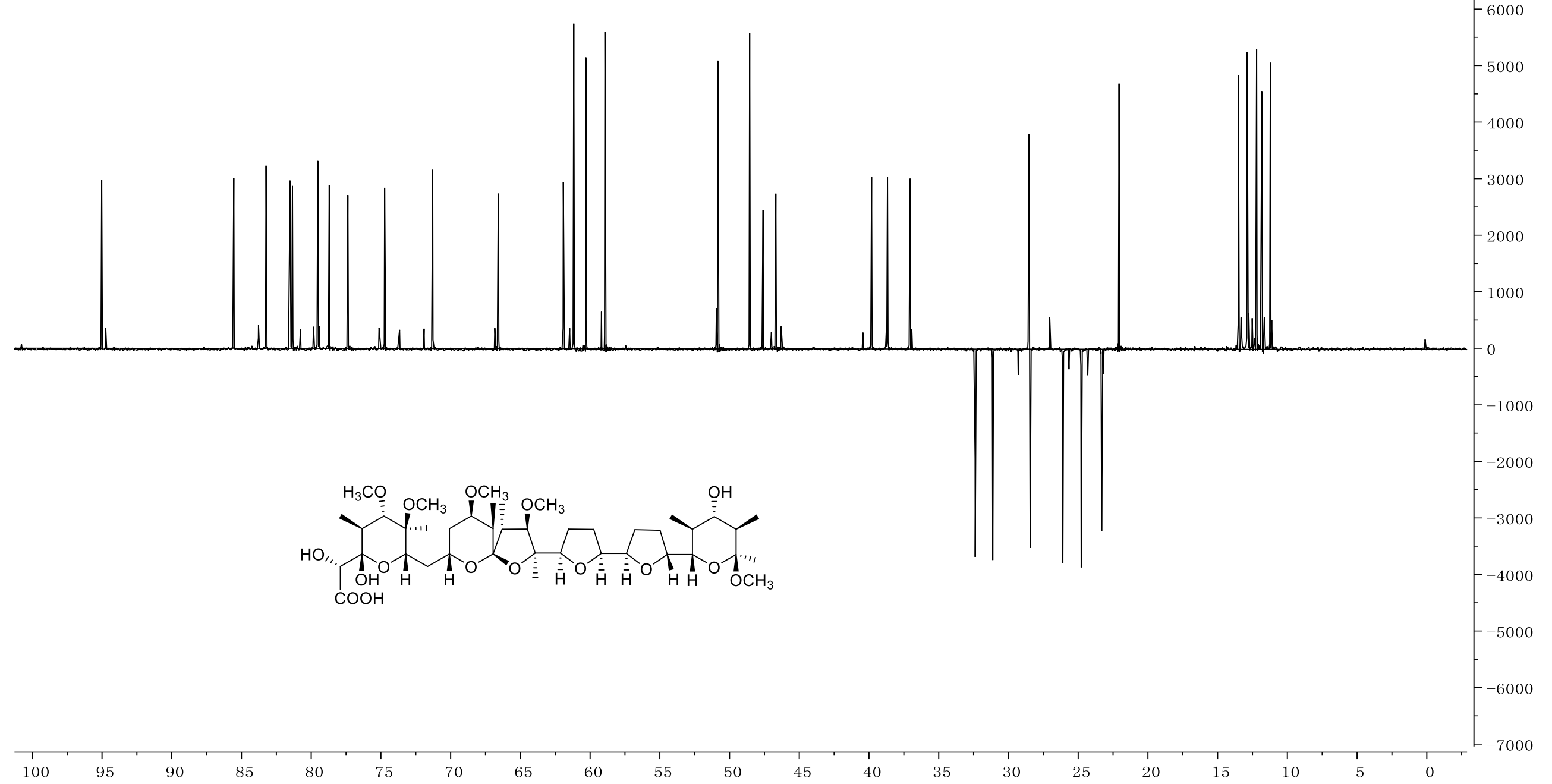


Figure S83. ${ }^{1} \mathrm{H}-{ }^{1} \mathrm{H}$ COSY spectrum of compound $\mathbf{1 0}$ in $\mathrm{CDCl}_{3}$.

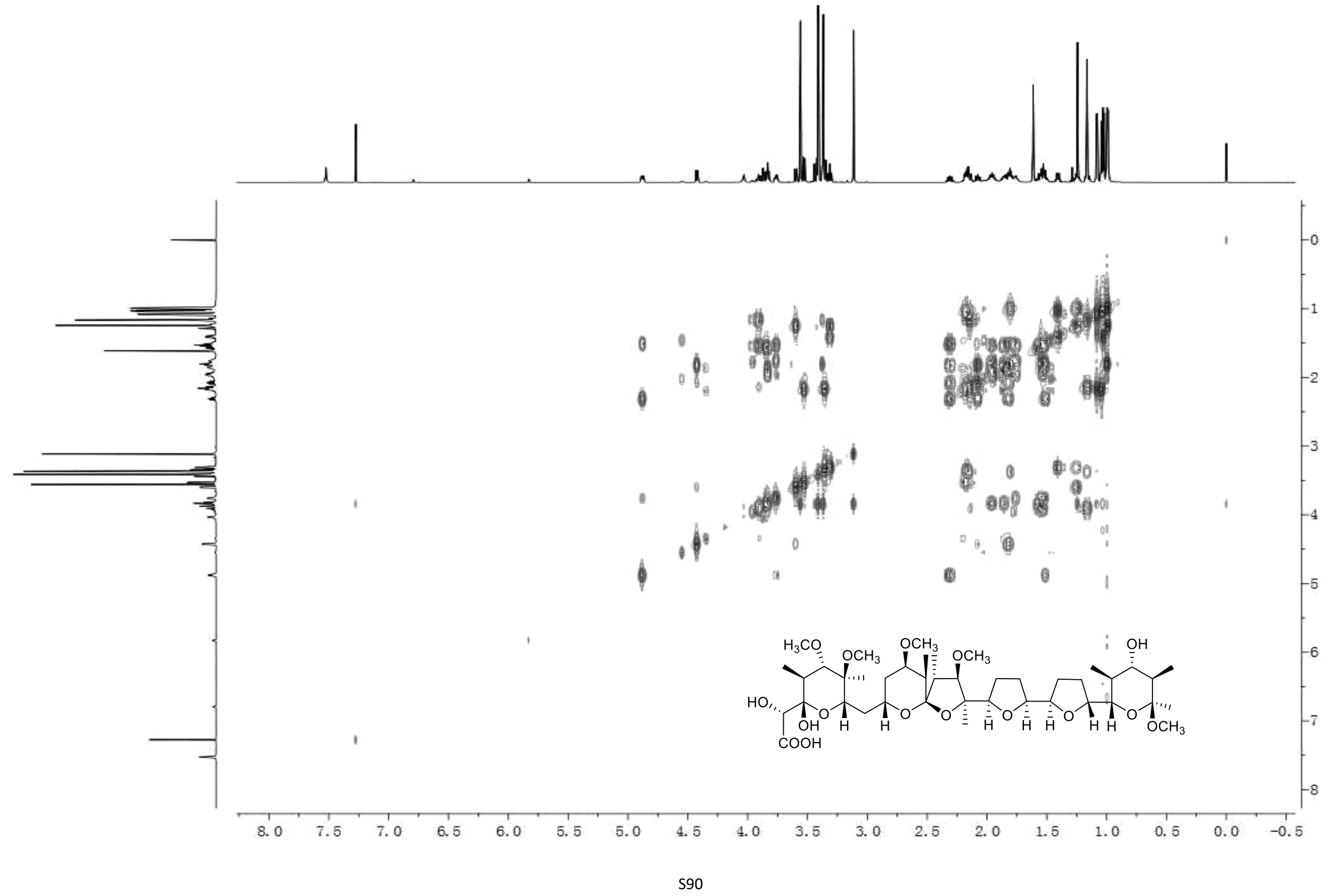


Figure S84. HSQC spectrum of compound 10 in $\mathrm{CDCl}_{3}$.

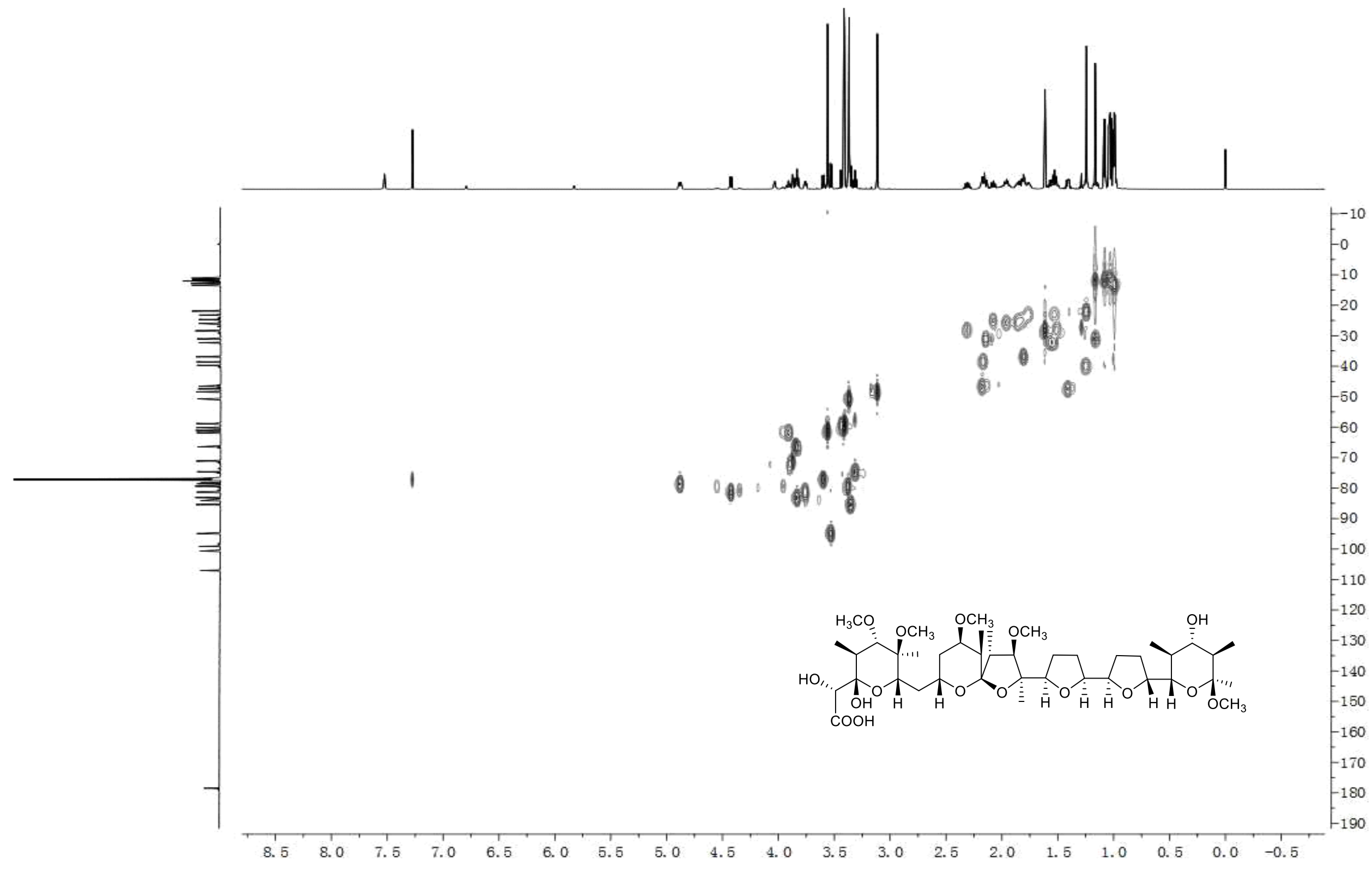


Figure S85. $\mathrm{HMBC}$ spectrum of compound $\mathbf{1 0}$ in $\mathrm{CDCl}_{3}$.

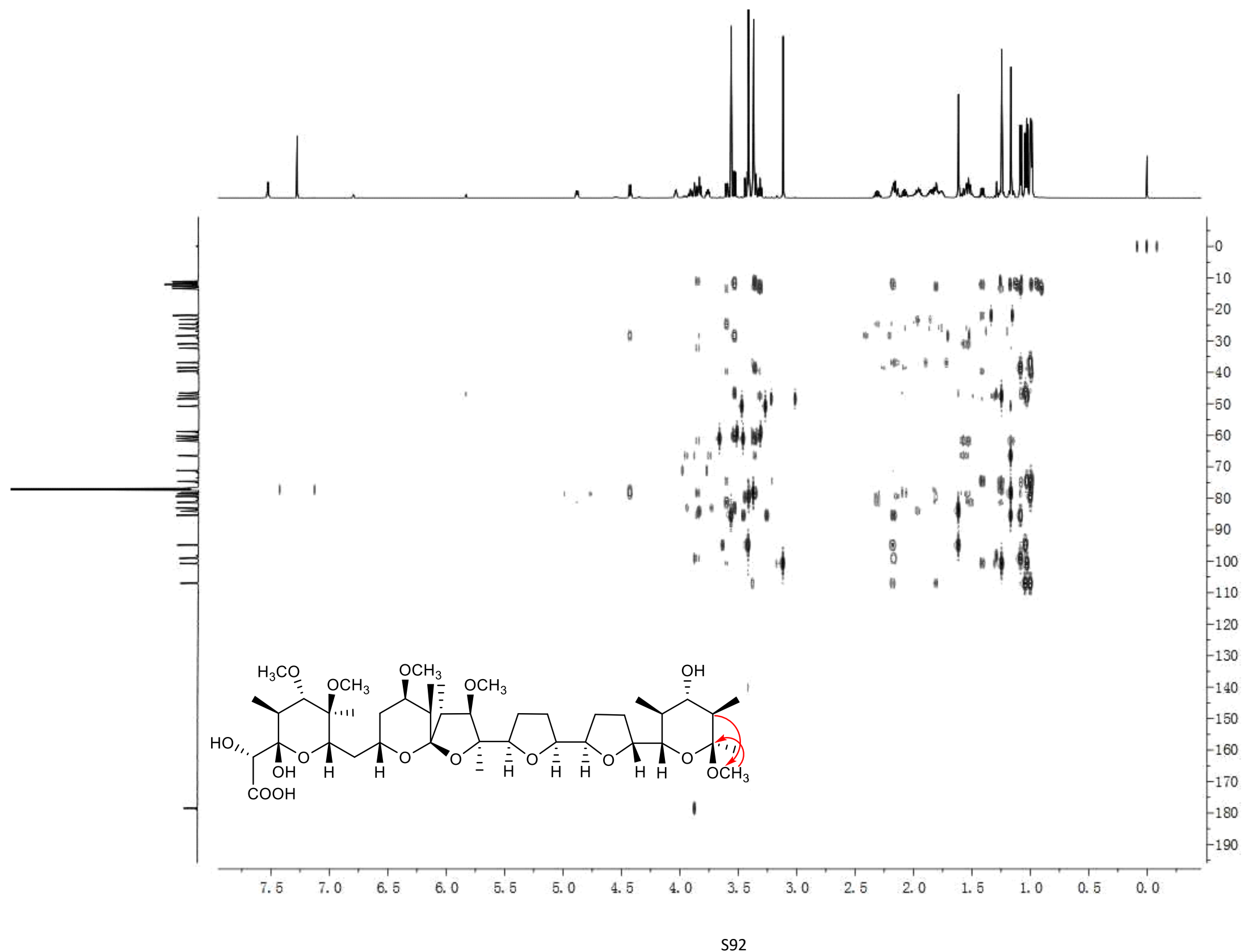


Figure S86. NOESY spectrum of compound $\mathbf{1 0}$ in $\mathrm{CDCl}_{3}$.

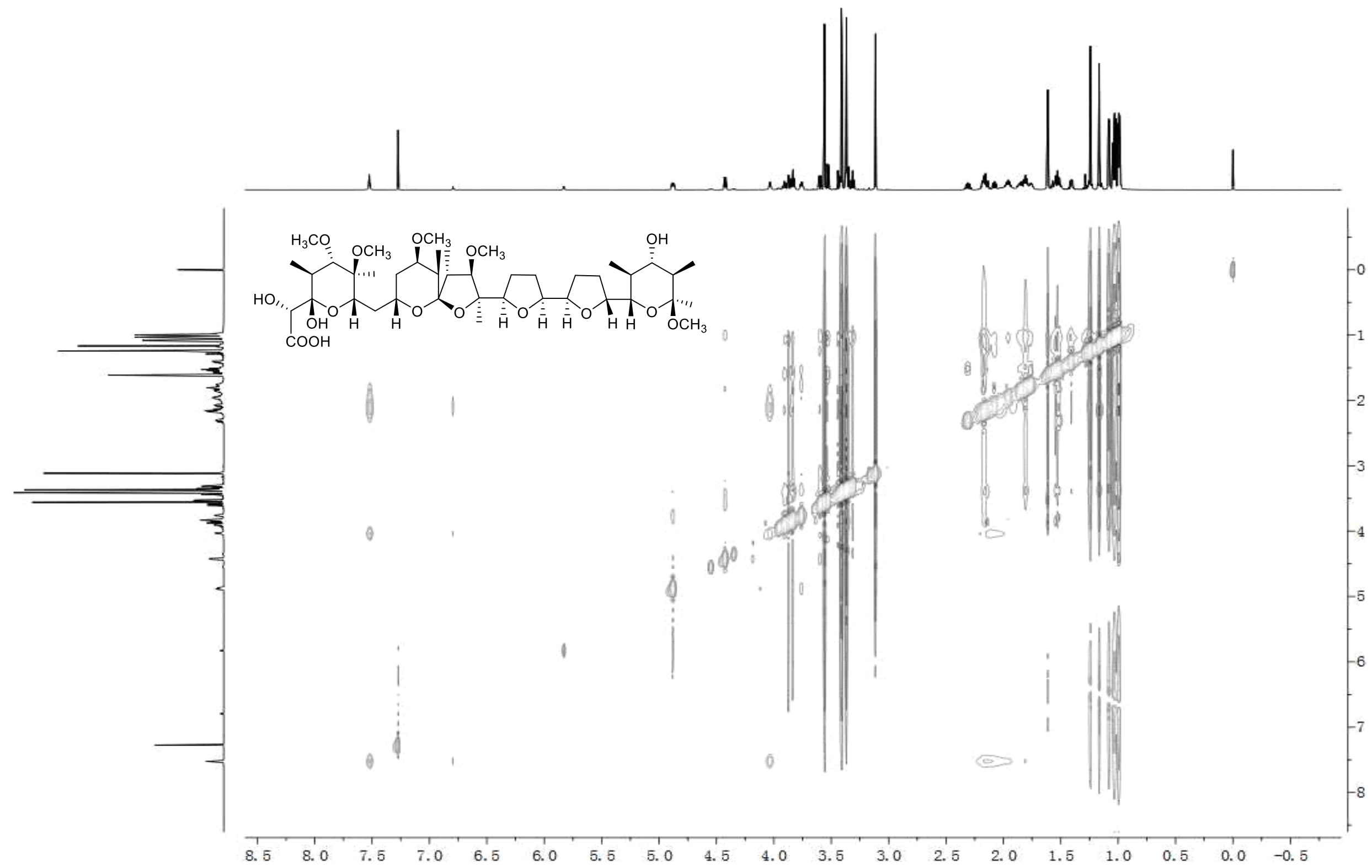


Figure S87. HR-ESI-MS spectrum of compound 11 in $\mathrm{MeOH}$.

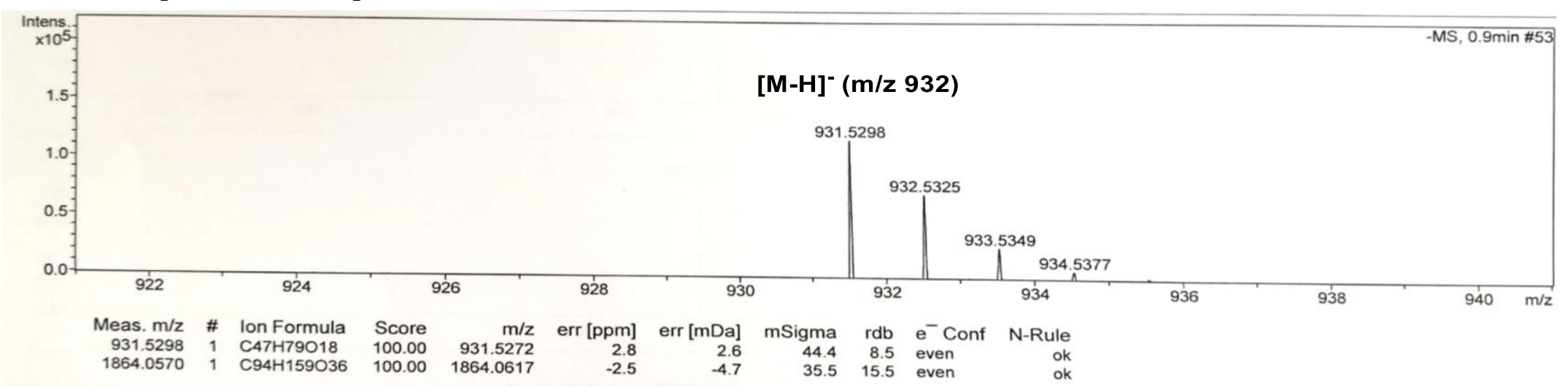

Figure S88. IR spectrum of compound 11.

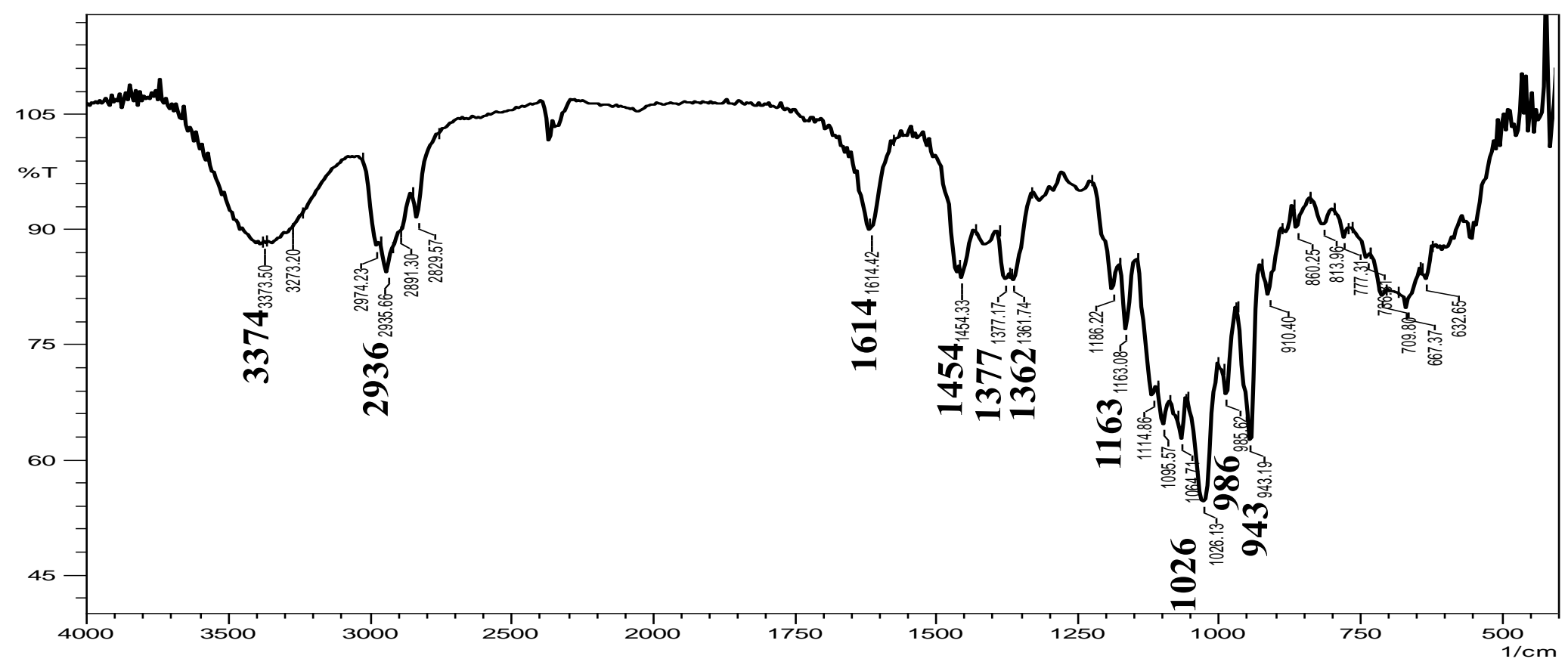


Figure S89. ${ }^{1} \mathrm{H}$ NMR $(700 \mathrm{MHz})$ spectrum of compound 11 in $\mathrm{CDCl}_{3}$.

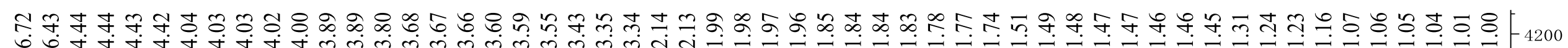

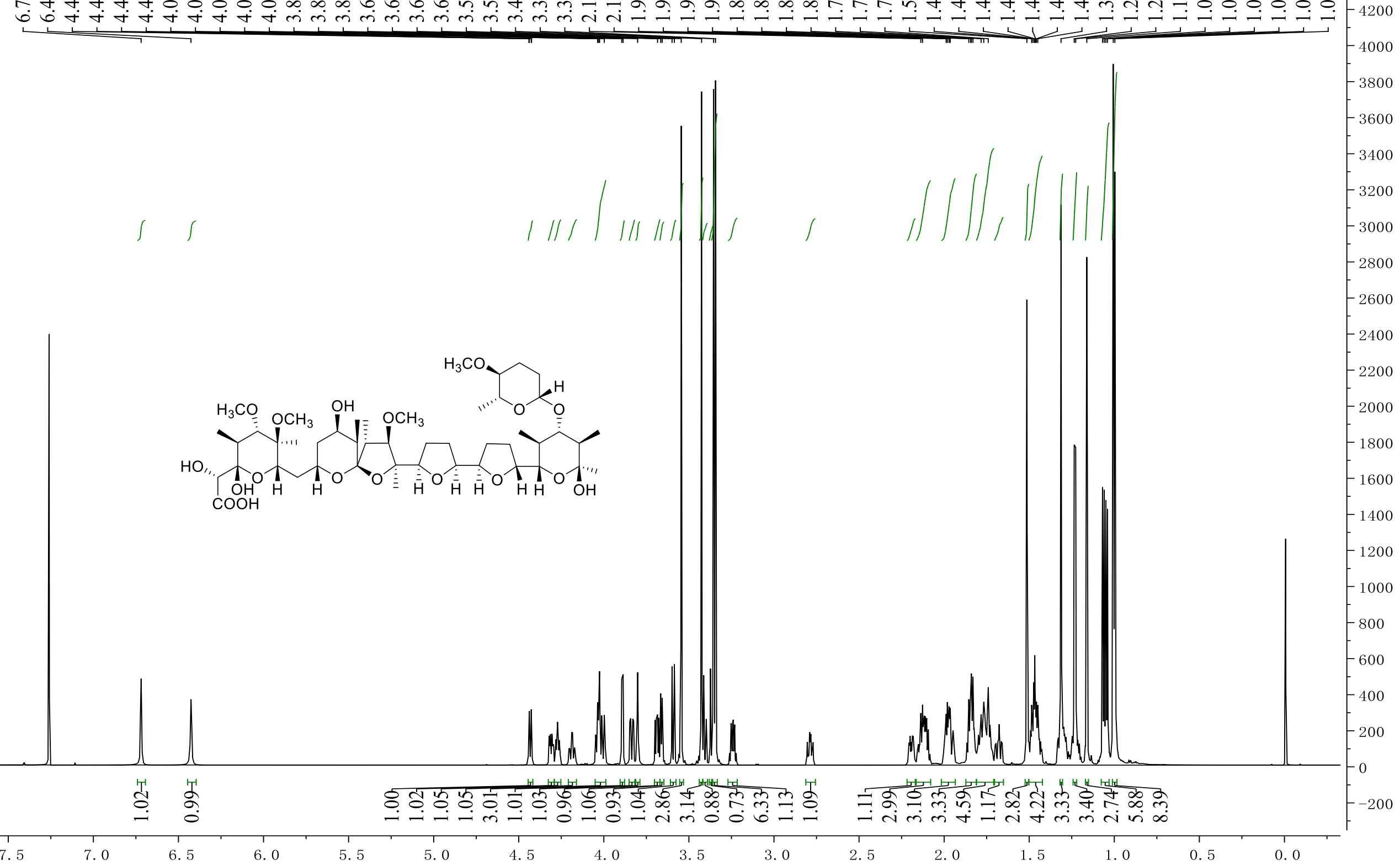


Figure S90. ${ }^{13} \mathrm{C}$ NMR $(175 \mathrm{MHz})$ spectrum of compound 11 in $\mathrm{CDCl}_{3}$.

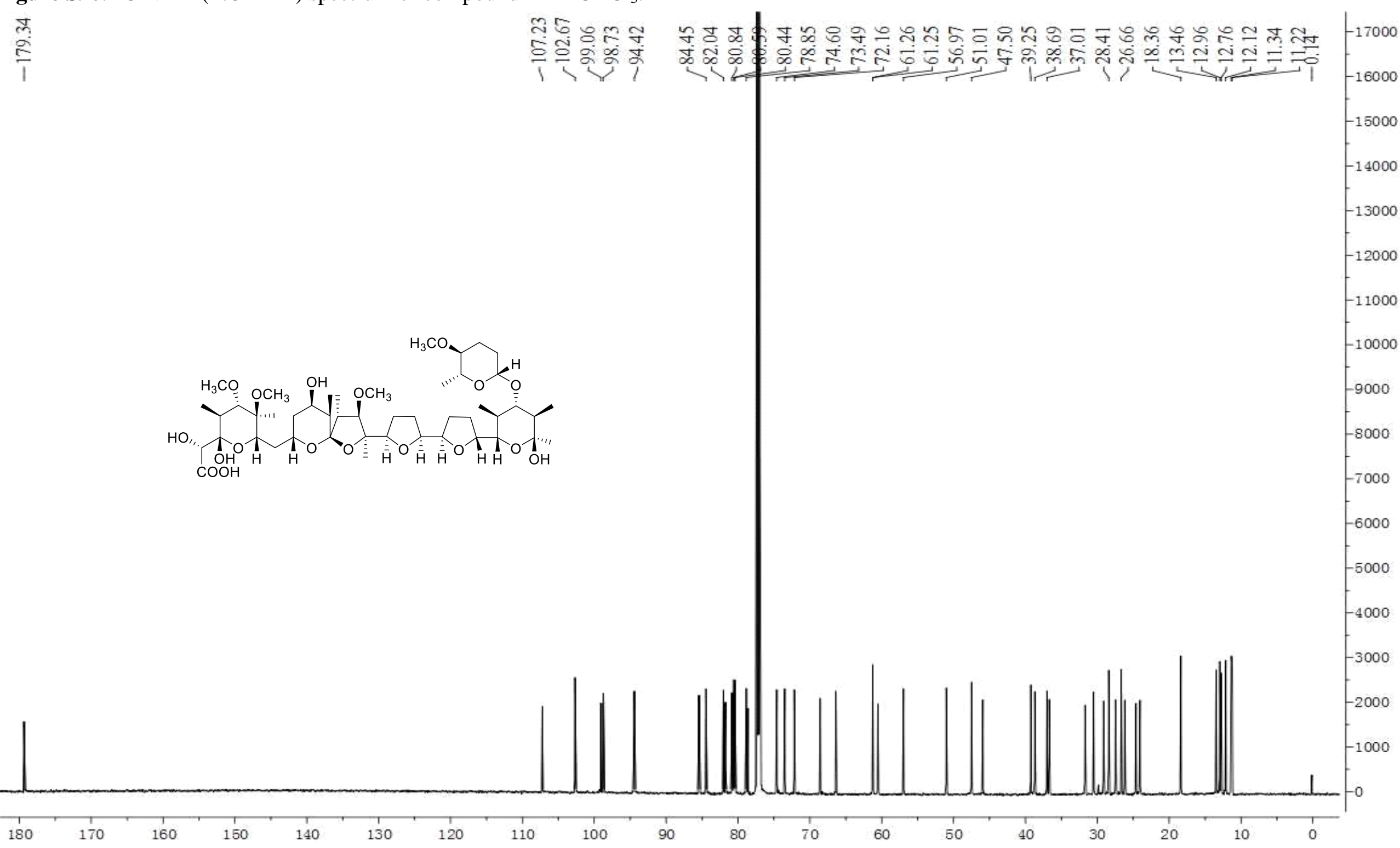


Figure S91. ${ }^{13} \mathrm{C}$ DEPT spectrum of compound 11 in $\mathrm{CDCl}_{3}$.

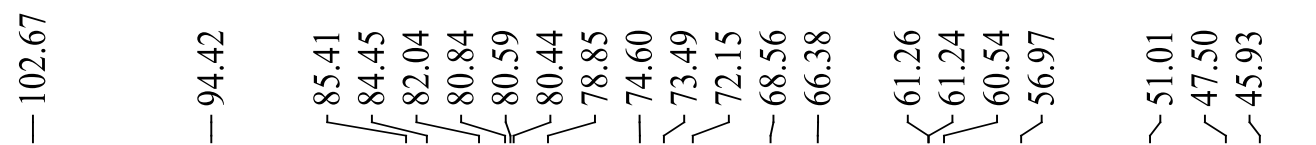

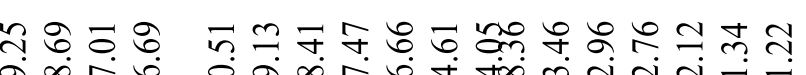

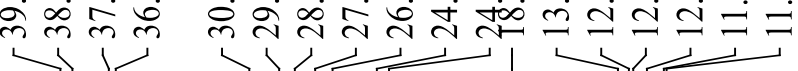

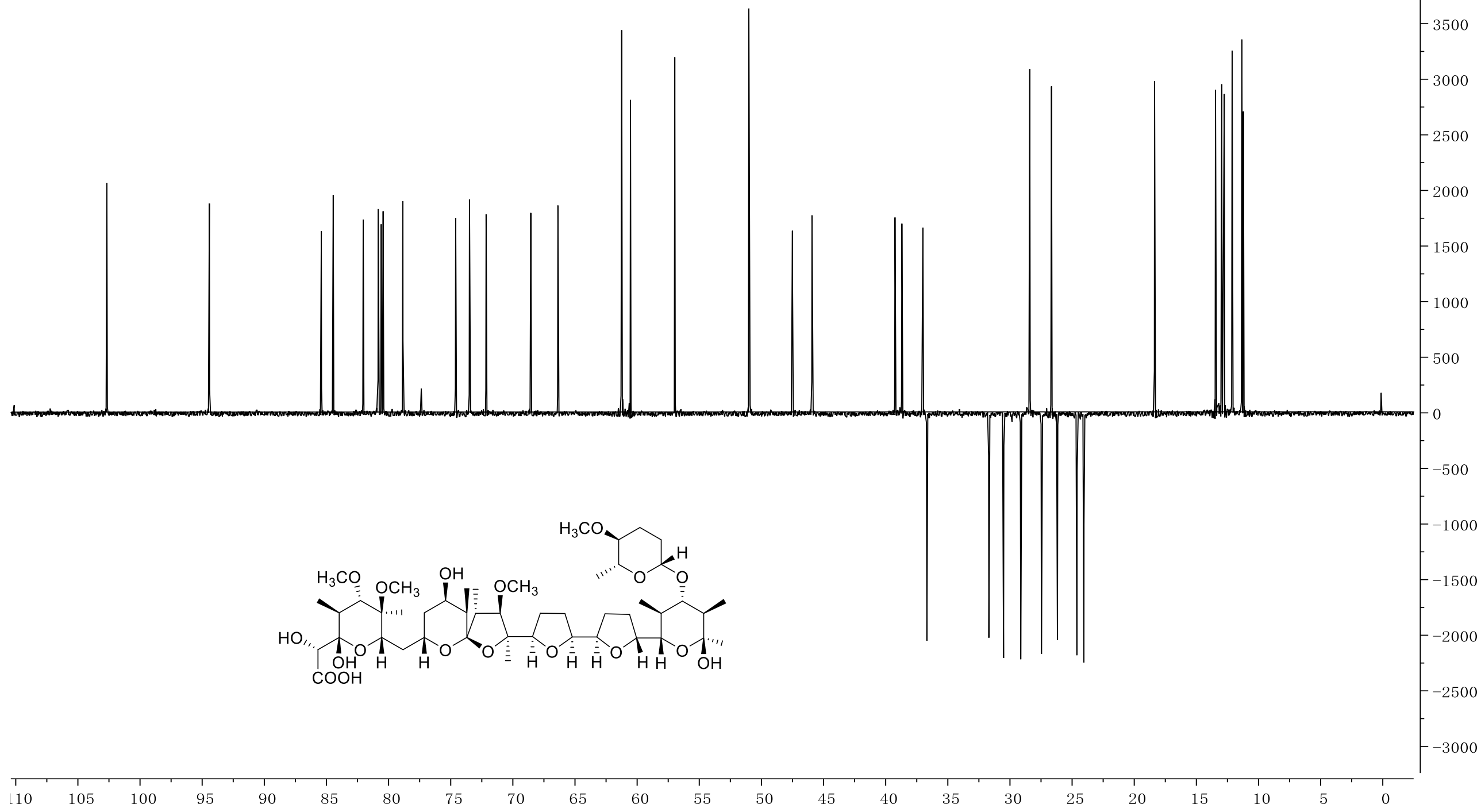


Figure S92. ${ }^{1} \mathrm{H}-{ }^{1} \mathrm{H}$ COSY spectrum of compound 11 in $\mathrm{CDCl}_{3}$.

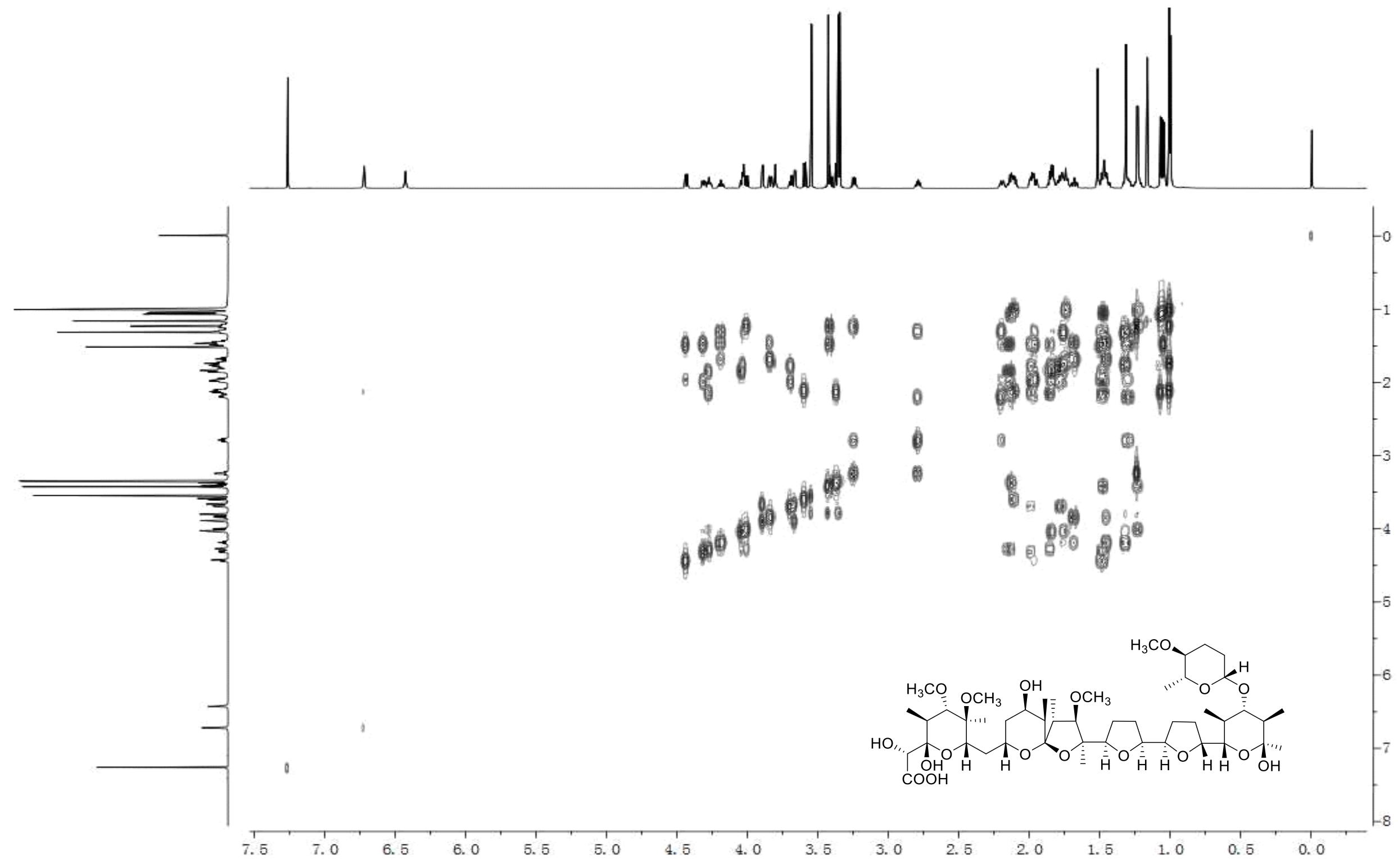


Figure S93. HSQC spectrum of compound 11 in $\mathrm{CDCl}_{3}$.

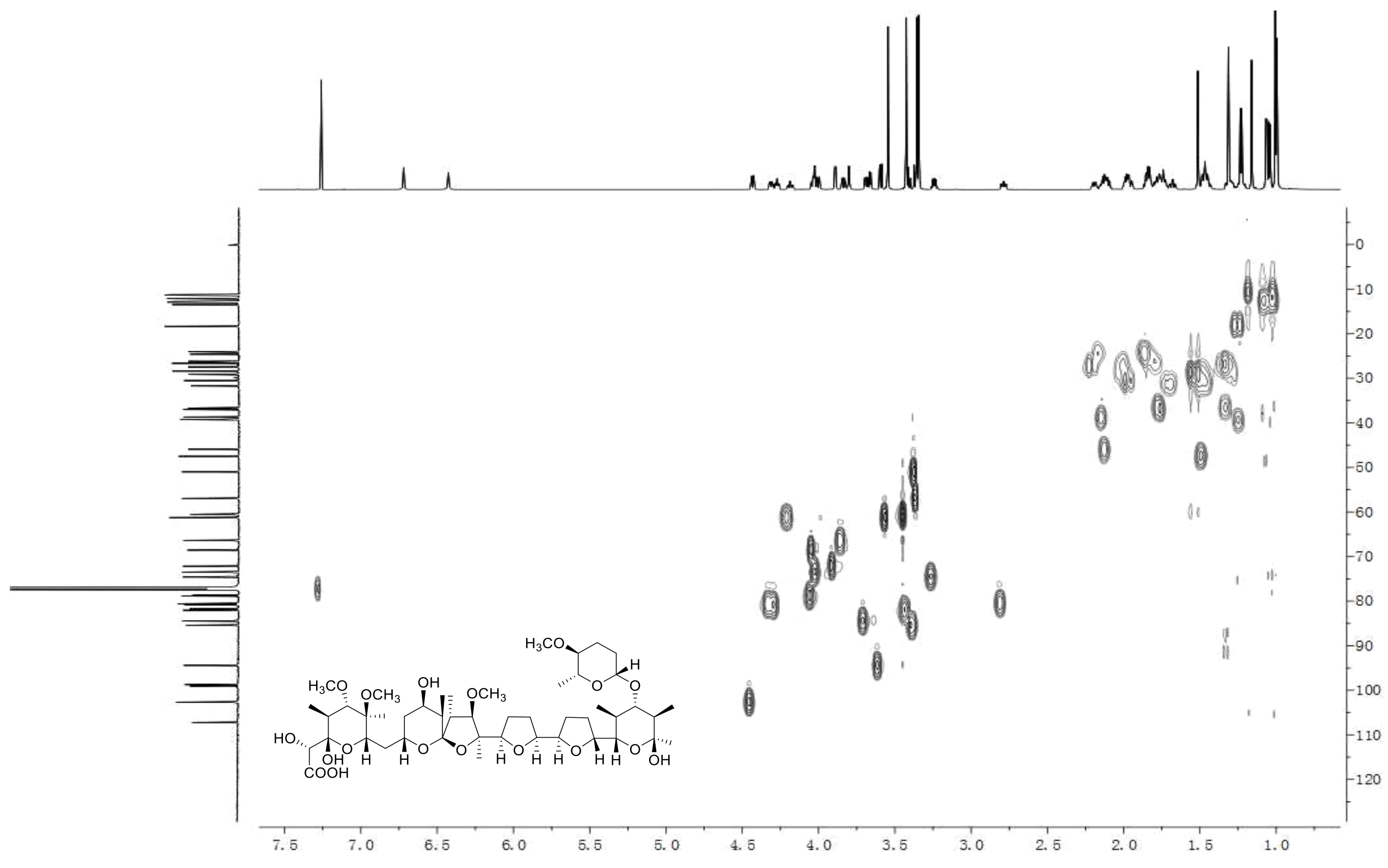


Figure S94. HMBC spectrum of compound 11 in $\mathrm{CDCl}_{3}$.

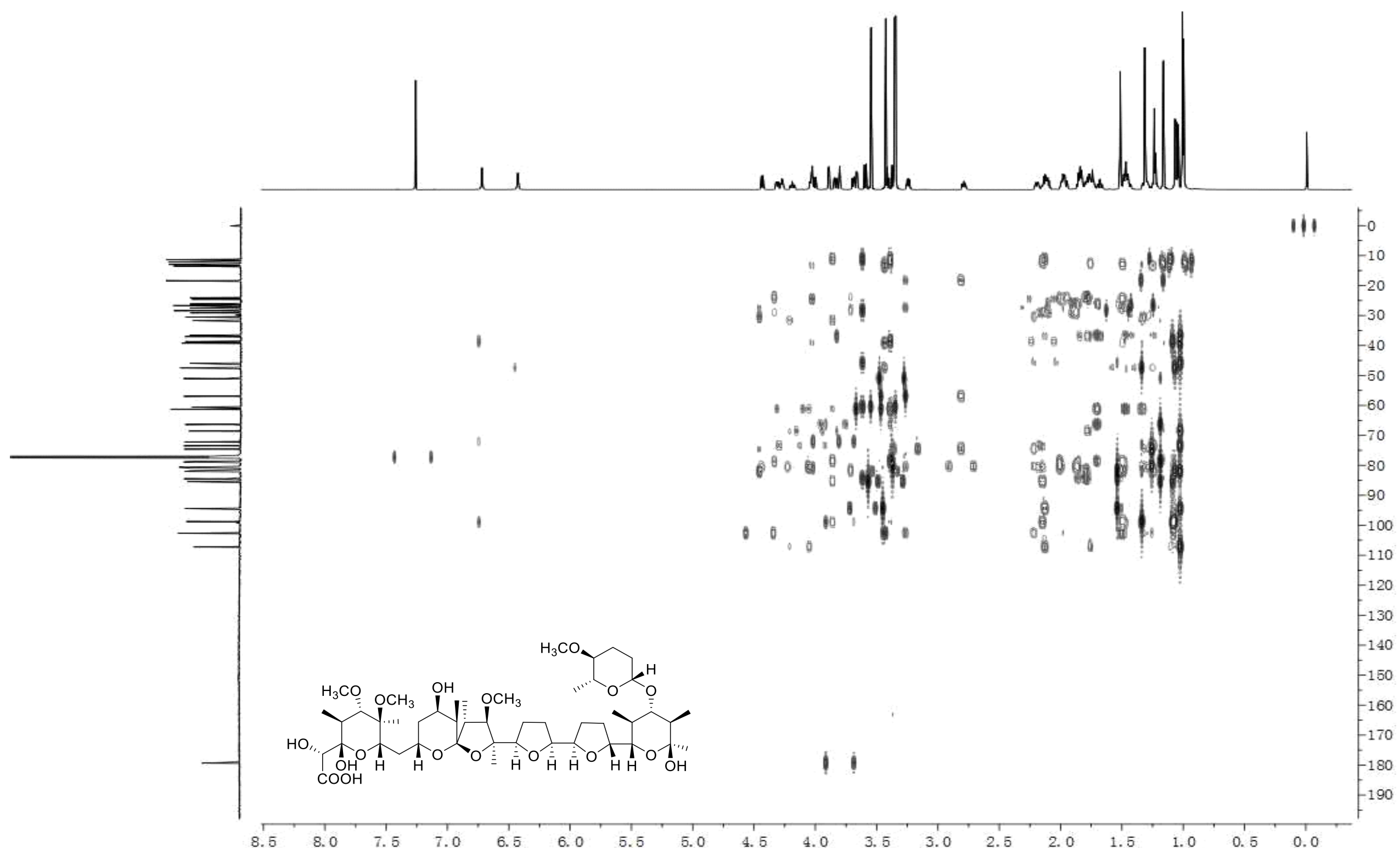


Figure S95. NOESY spectrum of compound 11 in $\mathrm{CDCl}_{3}$.

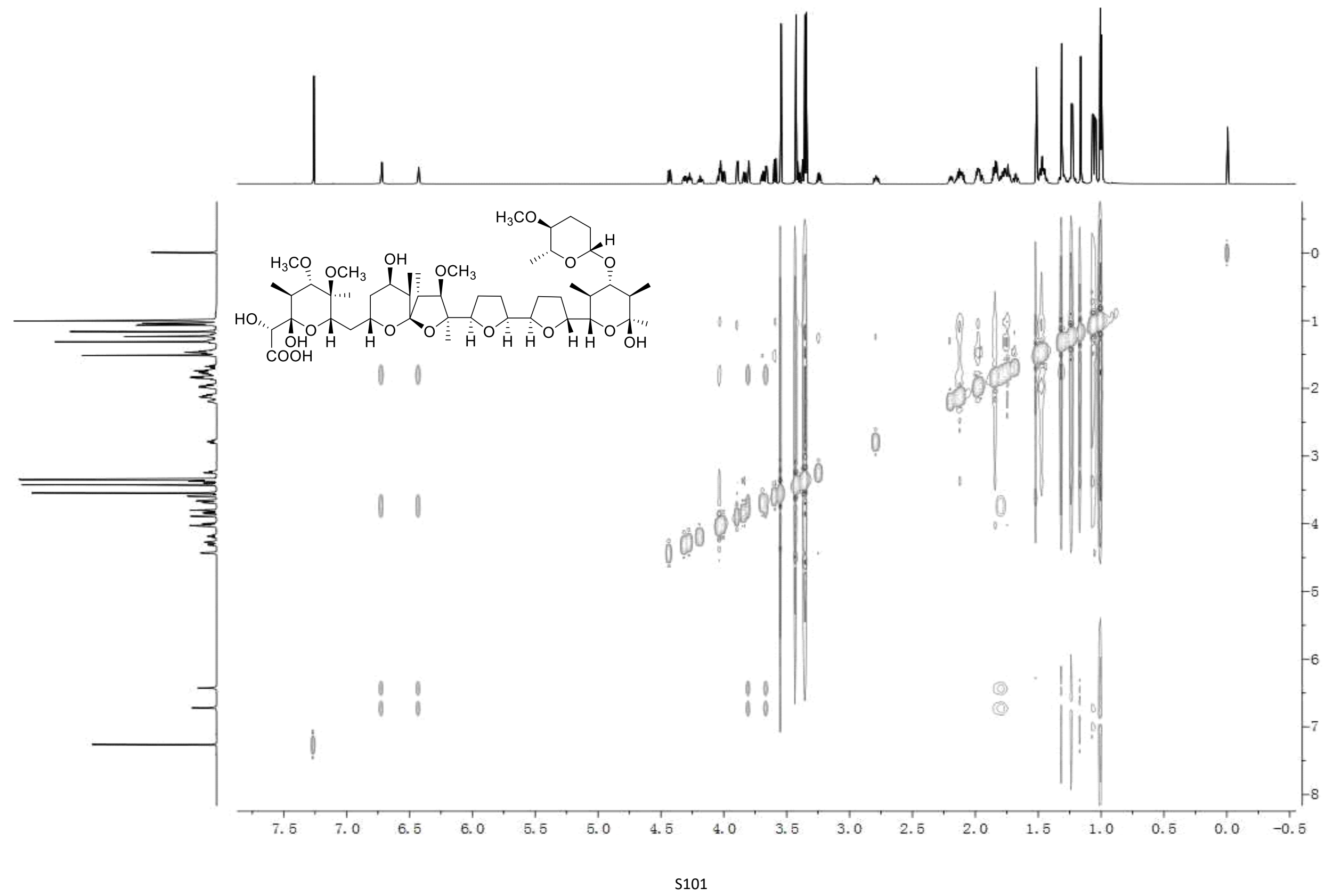




\section{References}

(1) MacNeil, D. J.; Gewain K. M.; Ruby, C. L.; Dezeny, G.; Gibbons, P. H.; MacNeil, T. Gene. 1992, $111,61-68$.

(2) Gust, B.; Chandra, G.; Jakimowicz, D.; Yuqing, T.; Bruton, C. J.; Chater, K. F. Adv. Appl. Microbiol. 2004, 54, 107-128.

(3) Datsenko, K. A.; Wanner, B. L. Proc. Natl. Acad. Sci. U.S.A. 2000, 97, 6640-6645.

(4) Paget, M. S.; Chamberlin, L.; Atrih, A.; Foster, S. J.; Buttner, M. J. J. Bacteriol. 1999, 181, 204-211.

(5) Tsuji, N.; Nagashima, K.; Terui, Y.; Tori, K. J. Antibiot. 1979, 32, 169-172. 